\title{
WHY DO WE ARGUE ABOUT SCIENCE? EXPLORING THE PSYCHOLOGICAL ANTECEDENTS OF REJECTION OF SCIENCE
}

\author{
JOHN RICHARD KERR
}

A thesis submitted to Victoria University of Wellington for the fulfilment of the requirements for the degree of Doctor of Philosophy

Victoria University of Wellington

2020 



\section{Abstract}

Science is recognised and accepted as an important tool for understanding the world in which we live, yet some people hold beliefs that go against the best available scientific evidence. For example, many people believe human-caused climate change is not occurring, or that vaccines are ineffective and dangerous.

Previous research has investigated a range of possible drivers of this 'rejection of science' (Lewandowsky, Oberauer, \& Gignac, 2013), including ignorance, distrust of scientists, and ideological motivations. The studies in this thesis extend this line of inquiry, focusing first on the role of perceptions of scientific agreement. I report experimental evidence that people base their beliefs on "what they think scientists think' (Study 1). However, an analysis of longitudinal data (Study 2) suggests that our personal beliefs may also skew our perceptions of scientific agreement. While the results of Study 1 and Study 2 somewhat conflict, they do converge on one finding: perceptions of consensus alone do not fully explain rejection of science.

In the remainder of the thesis I cast a wider net, examining how ideological beliefs are linked to rejection of science. Study 3 draws on social media data to reveal that political ideology is associated with rejection of science in the context of who people choose to follow on the platform Twitter. A final set of studies $(4,5$, and 6$)$ examine the role of two motivational antecedents of political ideology, Right-wing Authoritarianism (RWA) and Social Dominance Orientation (SDO), in rejection of science across five publicly debated issues. I also explore several potential mediators which might explain these effects. I report, for the first time, that RWA and SDO predict rejection of science across a range of issues and one mediator emerges as a consistent link: distrust of scientists. People who are less opposed to authoritarian (RWA) or hierarchical (SDO) values are less trusting of scientists and, in turn, more likely to reject specific scientific findings. I discuss potential strategies to address or circumvent this ideologically-motivated distrust of science.

Taken as whole, this thesis extends our understanding of why people disagree with an established scientific consensus on socially important issues. Knowledge of the scientific consensus matters, but our deeper beliefs about society can also draw us closer to, or push us further from evidence-based conclusions. 


\section{Table of Contents}

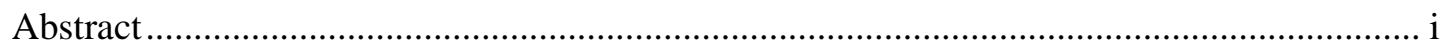

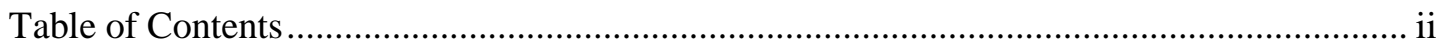

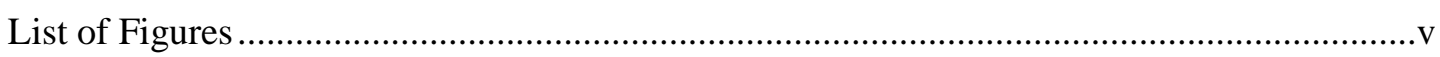

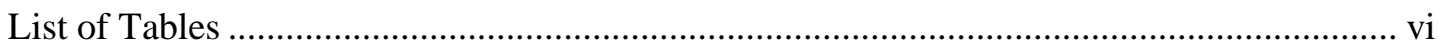

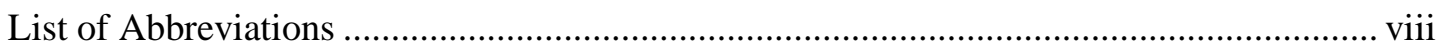

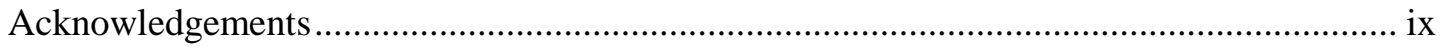

Chapter 1: Introduction .................................................................................................. 1

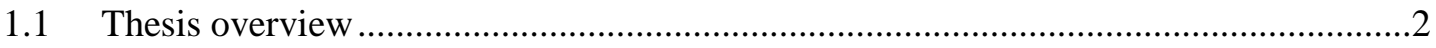

Chapter 2: $\quad$ Rejection of Science ............................................................................................ 5

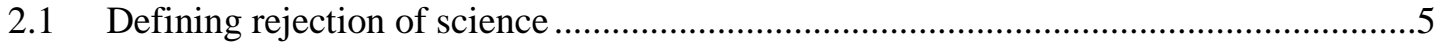

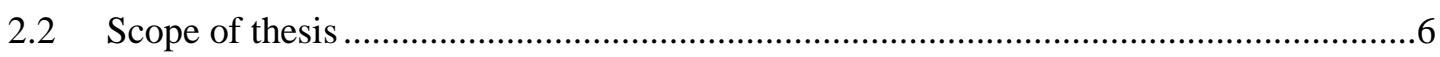

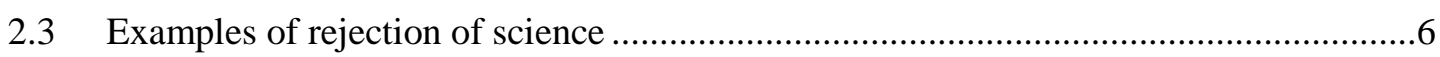

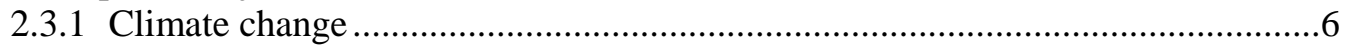

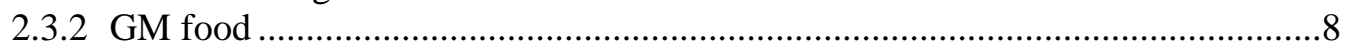

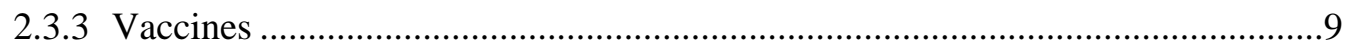

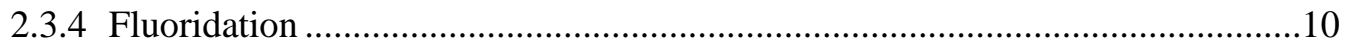

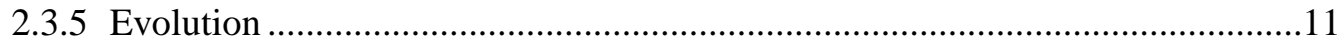

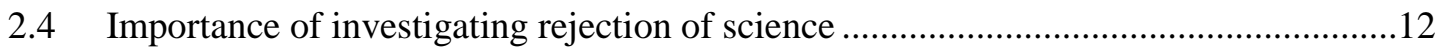

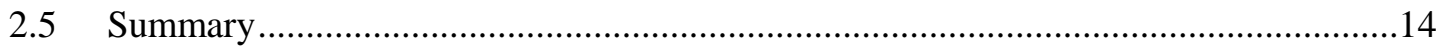

Chapter 3: Why Do People Reject Science? .................................................... 15

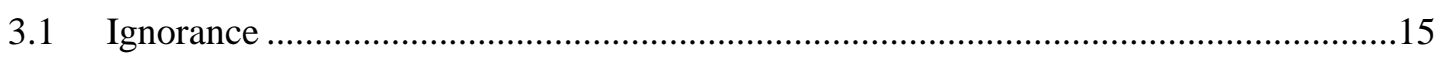

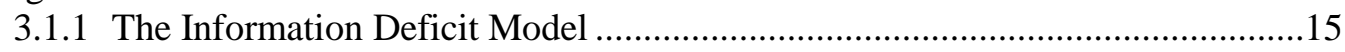

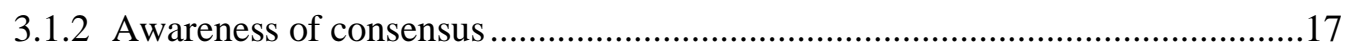

3.1.3 The Gateway Belief Model …….................................................................17

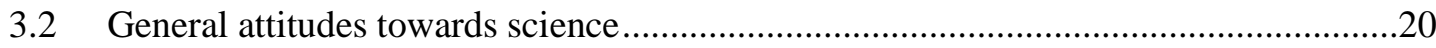

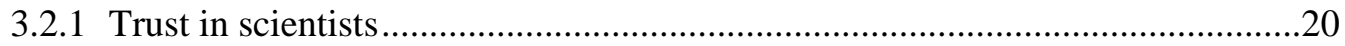

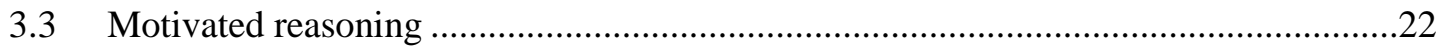

3.3.1 The Attitude Roots Model ............................................................................24

3.3.2 Attitude roots: Ideological predictors of rejection of science.............................25

3.3.3 The Dual Process Model: RWA and SDO as distal drivers of rejection of

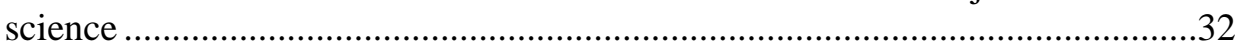

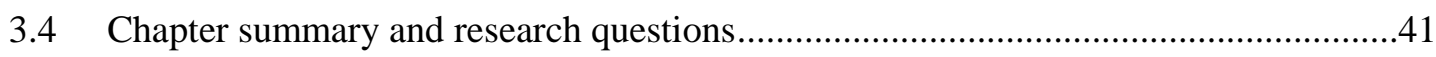

Chapter 4: Investigating the Gateway Belief Model............................................ 43

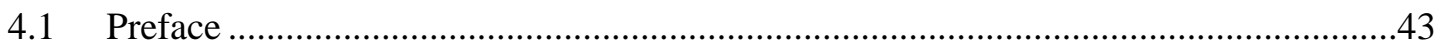

4.2 Study 1: Changes in perceived scientific consensus shift beliefs about climate change

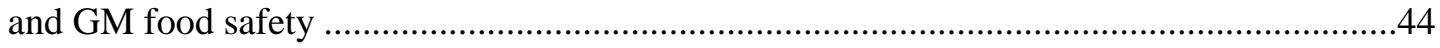

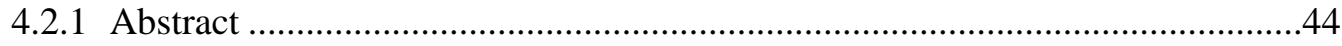

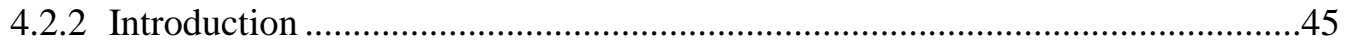

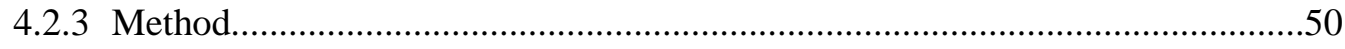

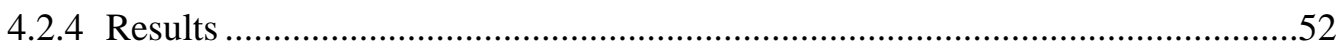


4.4 Study 2: Perceptions of scientific consensus do not predict later beliefs about the reality of climate change: A test of the gateway belief model using cross-lagged panel analysis.

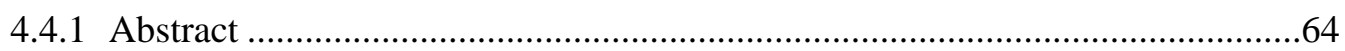

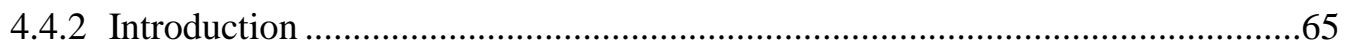

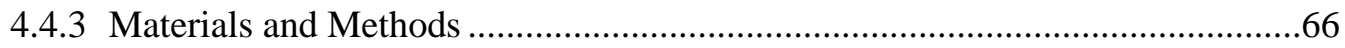

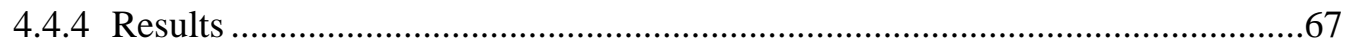

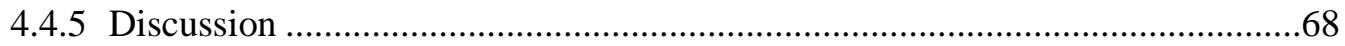

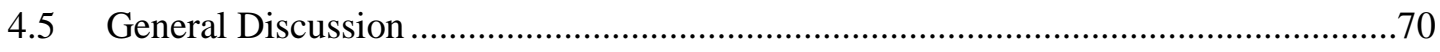

Chapter 5: Politics and Science in the New Zealand Twittersphere .............. 73

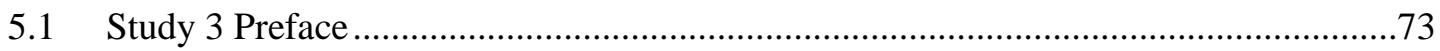

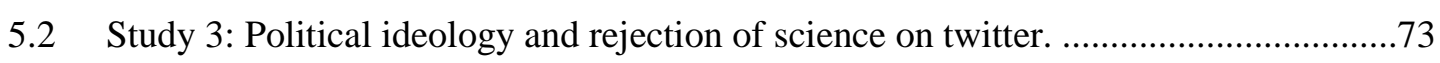

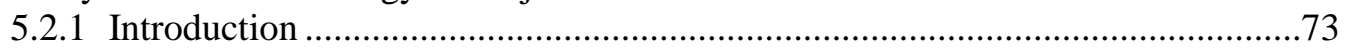

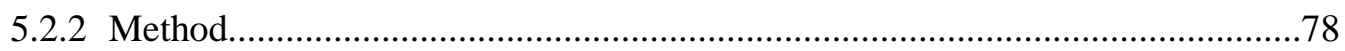

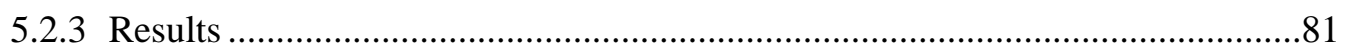

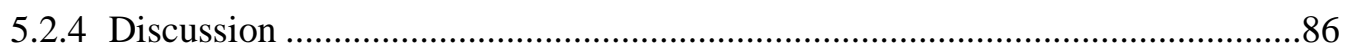

Chapter 6: The Role of RWA and SDO in Rejection of Science .................... 91

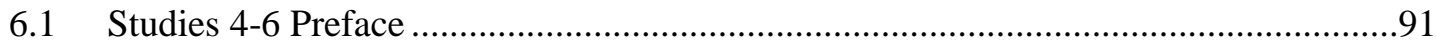

6.2 Study 4: Do ideological attitudes predict rejection of science? A study of New Zealand

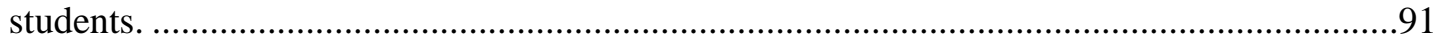

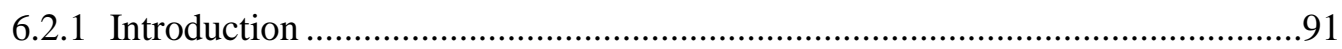

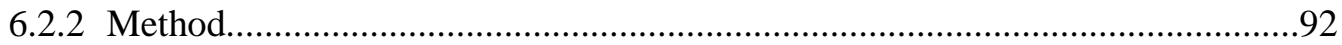

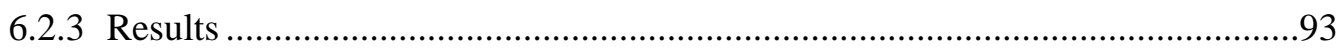

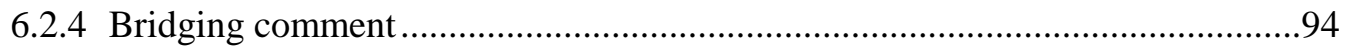

6.3 Study 5: Investigating the relationship between ideological attitudes and rejection of

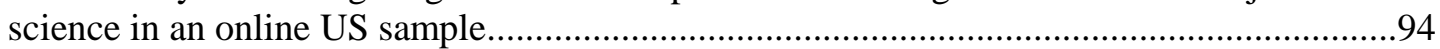

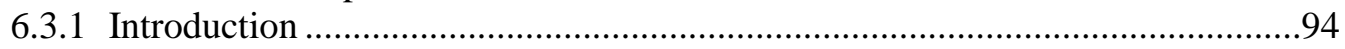

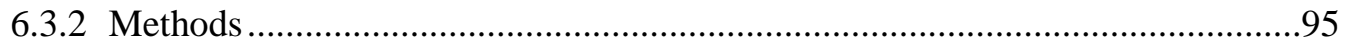

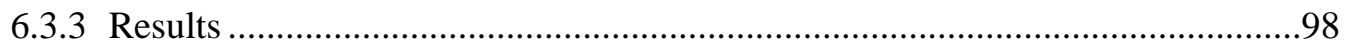

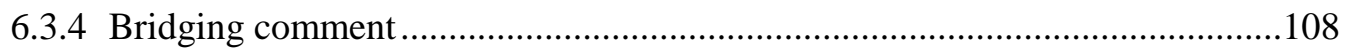

6.4 Study 6: Investigating the relationship between ideological attitudes and rejection of

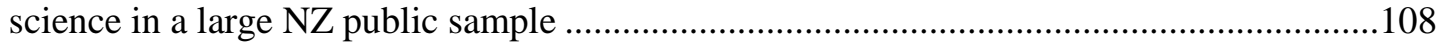

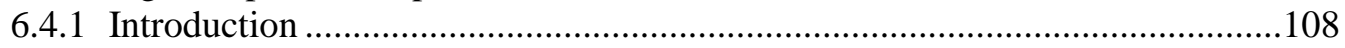

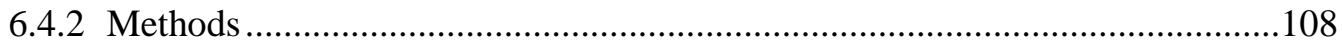

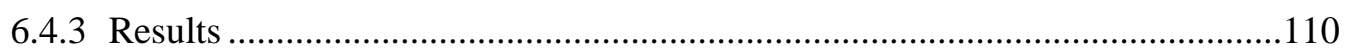

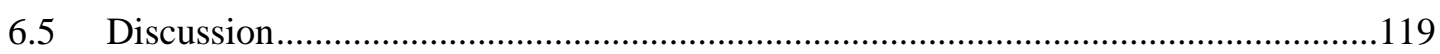

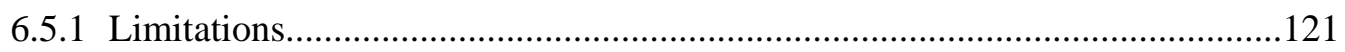

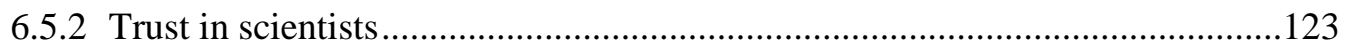

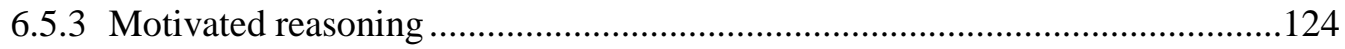

6.5.4 Practical implications for science communication .......................................125

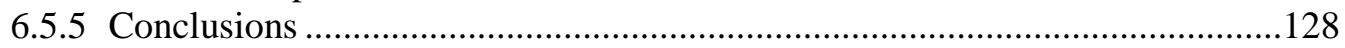

Chapter 7: $\quad$ Final discussion and conclusions.................................................... 129

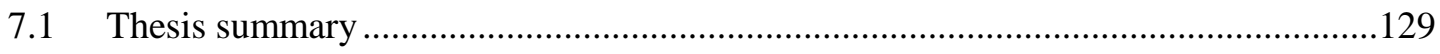

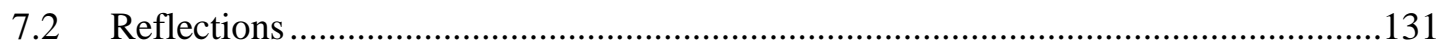

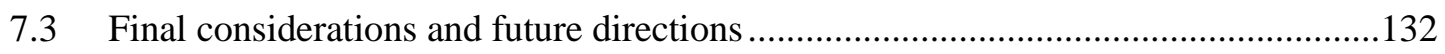


7.3.1 Extending the GBM.

7.3.2 Attitude roots and rejection of science on social media .................................133

7.3.3 Explaining the unexplained (variance) .........................................................134

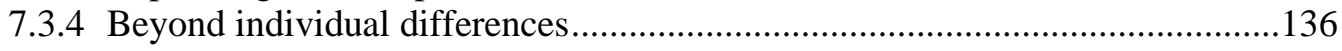

7.3.5 Jumping the attitude-behaviour gap .............................................................139

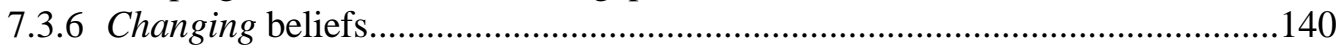

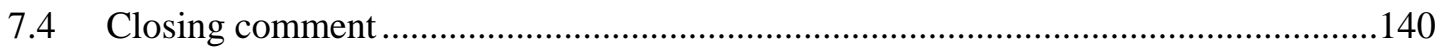

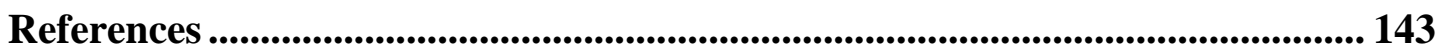

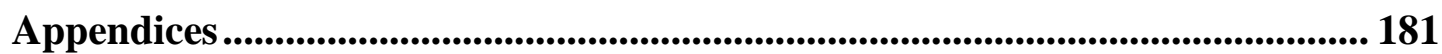




\section{List of Figures}

Figure 3.1. The Dual Process Model of Ideology....

Figure 4.1. Distribution of post-treatment estimates of scientific consensus on climate change (A) and average score on climate change belief scale in experimental conditions (B).

Figure 4.2. Distribution of post-treatment estimates of scientific consensus on GM food safety (A) and average score on GM food safety beliefs scale in experimental conditions (B).....

Figure 4.3. The effect of a $97 \%$ climate message on belief in human-caused climate change

Figure 4.4. The effect of a $63 \%$ climate message on belief in human-caused climate change

Figure 4.5. The effect of a 97\% GM message on belief that GM food is safe to eat

Figure 4.6. Standardized associations between personal beliefs and perceptions of consensus over time regarding the reality of ACC.

Figure 5.1. Percentage of NZ political Twitter account followers who also follow science sceptic accounts.

Figure 5.2. Average p-score for followers and non-followers of science sceptic accounts

Figure 6.1. Mediators of the effects of RWA and SDO on acceptance of science (Study 5).

Figure 6.2. Mediators of the effects of RWA and SDO on a second order latent science acceptance variable (Study 5).

Figure 6.3. Mediators of the effects of RWA and SDO on acceptance of science (Study 6).

Figure 6.4. Mediators of the effects of RWA and SDO on a second order latent science acceptance variable (Study 6).

Figure E.1. Percentage of NZ political party Twitter account followers who also follow science sceptic accounts. 


\section{List of Tables}

Table 4.1 Mean estimates of consensus among climate scientists (SD) before and after reading climate change consensus messages.

Table 4.2 Mean estimates of consensus among food scientists (SD) before and after reading GM consensus messages.

Table 5.1 Inclusion search terms for coding tweet relevance.

Table 5.2 Number of accounts in, and unique followers of each list used in the study.

Table 5.3 Number and percentage of liberal and conservative account followers who do or do not follow science sceptic accounts.

Table 5.4 Left-right scores for New Zealand political parties based on Jahn, Düpont, and Rachuj's (2018) analysis.

Table 6.1 Study 4 descriptive statistics and correlations.

Table 6.2 Demographic and ideological predictors (correlates) of acceptance of science.

Table 6.3 Study 5 descriptive statistics and correlations.

Table 6.4 Demographic and ideological predictors of acceptance of science (US sample).

Table 6.5 Total effects of RWA and SDO on acceptance of science latent variables (Study 5)

Table 6.6 Indirect, direct and total effects of RWA and SDO on overall science

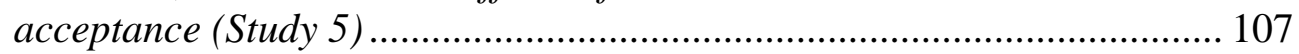

Table 6.7 Study 6 descriptive statistics and correlations.

Table 6.8 Demographic and ideological predictors of acceptance of science (NZ sample).

Table 6.9 Total effects of RWA and SDO on acceptance of science latent variables (Study 6)

Table 6.10 Indirect, direct and total effects of RWA and SDO on overall science acceptance (Study 6)

Table A.1 Study 1 measures.

Table B.1 Descriptive statistics for climate-related outcomes after reading a high or low climate consensus message or no message.

Table B.2 Descriptive statistics for GM-related outcomes after reading a high GM consensus message or no message.

Table C.1 Effect of 97\% climate consensus message on climate beliefs mediated via perceptions of consensus.....

Table C. 2 Effect of $63 \%$ climate consensus message on climate beliefs mediated via perceptions of consensus. 
Table C.3 Effect of 97\% GM consensus message on climate beliefs mediated via perceptions of consensus....

Table C.4 Effect of 97\% GM consensus message on GM food safety beliefs mediated via perceptions of consensus (low GM consensus and no message conditions combined as control group).

Table D.1 Study 2 measures.

Table D.2 Descriptive statistics and correlation matrix for all variables in cross-lagged model

Table D.3 Descriptive statistics and correlation matrix for participants with liberal voting intentions....

Table D.4 Descriptive statistics and correlation matrix for participants with conservative voting intentions.

Table D.5 Unconstrained beta weights for auto-regressive and cross-lagged paths and model fit when parameter constrained.

Table E.1 Overlap between followers of science sceptic accounts and followers of specific political parties....

Table E.2 Post hoc comparisons of the proportion of climate sceptic followers between parties.

Table E.3 Post hoc comparisons of the proportion of vaccine sceptic followers between parties.

Table E.4 Post hoc comparisons of the proportion of GM sceptic followers between parties.

Table F.1 Climate sceptic accounts and descriptions..... 203

Table F.2 GM sceptic accounts and descriptions .... 204

Table F.3 Vaccine sceptic accounts and descriptions 206

Table G.1 Standardised estimates of main model regression parameters (Study 5, US sample)

Table G.2 Indirect, direct and total effects in main model (Study 5, US sample)... 213

Table G.3 Standardised estimates of regression parameters in second-order science acceptance model (Study 5, US sample)

Table H.1 Standardised estimates of main model regression parameters (Study 6, NZ sample)

Table H.2 Indirect, direct and total effects in main model (Study 6, NZ sample)... 219

Table H.3 Standardised estimates of regression parameters in second-order science acceptance model (Study 6, NZ sample) 


\section{List of Abbreviations}

$\begin{array}{ll}\text { ACC } & \text { Anthropogenic climate change } \\ \text { AIDS } & \text { Acquired immune deficiency syndrome } \\ \text { API } & \text { Application programming interface } \\ \text { COSS } & \text { Credibility of Science Scale } \\ \text { CO }_{2} & \text { Carbon dioxide } \\ \text { DPM } & \text { Dual process model } \\ \text { GBM } & \text { Gateway belief model } \\ \text { HIV } & \text { Human Immunodeficiency virus } \\ \text { IPCC } & \text { Intergovernmental Panel on Climate Change } \\ \text { MP } & \text { Member of Parliament } \\ \text { NZ } & \text { New Zealand } \\ \text { OLS } & \text { Ordinary least squares } \\ \text { RWA } & \text { Right-wing authoritarianism } \\ \text { SDO } & \text { Social dominance orientation } \\ \text { SEM } & \text { Structural equation modelling } \\ \text { US } & \text { United States }\end{array}$




\section{Acknowledgements}

My first thanks must go to Prof Marc Wilson. I could not have asked for a more knowledgeable, patient, and kind supervisor. It is only with hindsight that I can appreciate Marc's skill in guiding his students. He gave me the freedom to explore a multitude of avenues while offering near-imperceptible nudges to shift me away from potential pitfalls.

I also owe a great deal to my fellow PhD students in the School of Psychology, without their advice and support this thesis would have not been possible. Special thanks are owed to Dr Samantha Stanley, who put up with my incessant questions and messy desk, and to Johannes Karl for being an infinite source of statistical wisdom and interesting conversation.

I am sincerely grateful to the academics who gave up their time to meet and correspond with me regarding my research, in particular: Dr Sander van der Linden and Prof Matthew Hornsey. Their advice and critique (and, as will become obvious, their research) have undoubtedly shaped this thesis. I must also acknowledge Prof Jean Fleming, who over the last decade has provided me with much support, and advice and good conversation. It was her initial encouragement that sent on me the first steps of this journey.

I would also like to acknowledge the financial support of the Victoria Doctoral Scholarship and Sasakawa Young Leaders' Fellowship Fund. Without this support, this thesis and my family would both be quite emaciated. In this regard, I am also grateful to the Centre for Science and Society and the Science Media Centre for gainful part-time employment during my studies.

Without the sustained support of friends and family, this would have been a much more difficult journey. I'm grateful to the many people have helped me along the way, particularly Samuel Murray, Veronica Stevenson, and Jared Humm for their valued friendship, advice and proofreading. Thanks are also due to my mother, brother and sisters who have supported me in my study and all the life changes occurring alongside it (read: new parenthood). I must also acknowledge my late father, Dr Alan Kerr, to whom I owe my thirst for knowledge. 
My last thanks go to my best friend and wife Katie Norton, who has been beside me every step of the way, and my daughter Athena-whose arrival in 2018 presented an ongoing and most welcome distraction from my studies. I love you both with all my heart. 


\section{Chapter 1: Introduction}

A habit of basing convictions upon evidence, and of giving to them only that degree of certainty which the evidence warrants, would, if it became general, cure most of the ills from which the world suffers.

—Bertrand Russell, Why I am not a Christian, 1927

In 2016, the Oxford Dictionary announced "post-truth" as their word of the year, defined as "relating to or denoting circumstances in which objective facts are less influential in shaping public opinion than appeals to emotion and personal belief." According to Oxford Dictionaries (n.d.), the word of the year is judged to "reflect the ethos, mood, or preoccupations of that particular year and to have lasting potential as a word of cultural significance."

The fact that 'post-truth' captures the zeitgeist of 2016 shows just how worried we are, as a society, about people holding beliefs which run counter to the best available evidence. This is particularly apparent at the intersection of science and society. Debates over climate change, vaccination and genetic modification are repeatedly cited as examples of opinion trumping facts. Amidst the editorial and comment pages of journals such as Science and Nature, leaders of the scientific community have expressed their worry over misinformation and the increasing number of battlegrounds upon which scientific expertise and public opinion square off (Druckman, 2017; Gewin, 2017; Hamburg, 2019; Lazer et al., 2018; "The challenge of the post-truth era," 2018). For example, half a decade before 'post-truth', a 2010 Nature editorial warned of "a growing anti-science streak... that could have tangible societal and political impacts on many fronts" (p. 133).

Leaders in New Zealand are also uneasy about the perceived rise in scepticism of scientific consensus. In 2017, the Chair of the New Zealand Environmental Protection Agency issued a warning in the agency's annual report:

Over recent years, there has been a groundswell of opposition to perceived bureaucracy and a growing scepticism about science and the role of so-called "experts". New Zealand is not immune to this - we have our share of science deniers, 
who oppose fluoride, 1080, vaccinations, glyphosate, genetic modification, and much more; and find their views reinforced and nurtured in the unmoderated milieu of the internet. (Environmental Protection Agency, 2017, p. 5)

Following the publication of the Agency's report, The Prime Minister's Chief Science Advisor, Professor Sir Peter Gluckman, was asked to respond to claims New Zealand has an anti-science problem. He said:

I think that is true... But whether it's true in New Zealand or not, beyond that general statement, it's difficult to know. Where is the formal evidence of it? ... I'm not sure there is any specific data on New Zealand. (Morton, 2017)

This thesis ambitiously aims to tackle the problem of "why do people argue about science?' On the one hand, we generally recognise science as the best source of information about the world we live in and the choices we have to make as individuals and societies. And yet, on the other hand, we also find ourselves in a time where, for certain specific claims, scientific evidence is routinely ignored and rejected by certain groups. In the process of addressing this question, I will offer an insight into the drivers of such counter-scientific beliefs. Of course, there are many situations where science cannot (yet) furnish a clear-cut answer for questions. The influence of violent videogames on children's behaviour is an example of a societally relevant body of research in which no clear-cut consensus has yet emerged (Ferguson \& Wang, 2019). However, this thesis—like much related researchfocusses on instances where the scientific evidence has become so overwhelmingly stacked on one side of a debate, that, objectively, the argument is all but closed. Understanding how and why people arrive at conclusions conflicting with such evidence is the aim of this thesis.

\subsection{THESIS OVERVIEW}

In the following chapter (Chapter 2) I offer a clear definition of rejection of sciencethe holding of beliefs in opposition to established scientific consensus - and provide five examples which are central to the original studies in this thesis. Here I also explain why studying rejection of science matters, and how such research can be applied in the real world.

In Chapter 3, I review several approaches to explaining rejection of science. For example, social psychologists have developed a model positing that perceptions of scientific consensus are integral to individuals' acceptance of scientific claims (van der Linden, Leiserowitz, Feinberg, \& Maibach, 2015). Others have focused on how biased reasoning 
allows political and religious beliefs to skew our beliefs on scientific issues, leading us further from the truth, inasmuch as science can capture it (Hornsey \& Fielding, 2017).

The next three chapters (4-6) cover six studies addressing questions raised by the literature reviewed in Chapter 3. Firstly, in Chapter 4 I explore how our beliefs about 'what scientists think' influence our own opinions on scientific issues. The two studies in this chapter shed light on how perceptions of scientific consensus influence our beliefs about publicly debated science: if we believe that most scientists agree $\mathrm{X}$ is true, will we believe $\mathrm{X}$ is true? While Study 1 (Kerr \& Wilson, 2018a) furnishes some experimental evidence that this is the case, Study 2 (Kerr \& Wilson, 2018b) finds that this relationship is more complicated, with longitudinal evidence suggesting that we may base our perceptions of scientific consensus more on our own beliefs rather than vice versa. Ultimately, while perceptions (or misperceptions) of scientific consensus may explain some variation in our views on issues such as climate change or GM food safety, deeper psychological factors also influence our opinion on such controversial issues (Hornsey \& Fielding, 2017; Lewandowsky \& Oberauer, 2016).

Over the course of my thesis research, my focus shifted to these more distal drivers of rejection of science. Chapters 5 and 6 reflect this change in trajectory, delving into 'ideological' motives for rejecting the scientific consensus. In Chapter 5, I present evidence that political ideology is linked with rejection of science, based on behavioural data from the social media platform Twitter (Study 3). This study finds that people who follow the Twitter accounts of New Zealand right-wing parties and politicians are more likely to follow Twitter accounts which challenge the scientific consensus on climate change, vaccines, and, in some cases, GM food, compared to people who follow the twitter accounts of New Zealand leftwing parties and politicians. This study foreshadows the findings of subsequent survey-based studies $(\mathbf{4}, \mathbf{5}$, and 6) in Chapter 6 which reveal that self-reported political conservatism is correlated with rejection of the scientific consensus across a range of issues in both the US and New Zealand. However, these final studies go far beyond examining just political ideology. In reviewing the various intertwined predictors of rejection of science I make a case that deeper beliefs about authority, tradition and hierarchy may explain rejection of science in a broader sense. Using structural equation modelling (SEM) I show that the ideological attitudes' right-wing authoritarianism (RWA) and social dominance orientation (SDO) predict rejection of science across different scientific domains relevant to the lives of everyday people. I also find that the reasons for these associations vary; ideological factors, 
such as political conservatism or conspiracy thinking, differentially explain the effects of ideological attitudes on our beliefs about different scientific issues. One factor emerges clearly as a consistent link between RWA, SDO and rejection of science: trust in scientists. Individuals who embrace authoritarian or hierarchical values are less likely to trust scientists, and those who trust scientists less are less likely to accept the consensus position of scientists on any of the issues examined. I then reflect on how the results of these studies expand psychological models of ideology and rejection of science. I also offer some practical insights on how scientists and policymakers can apply these findings in efforts to engage with the public on controversial and politicised scientific issues.

In a brief final discussion (Chapter 7), I summarise the research presented and offer some final thoughts on how the findings can inform future research and advance our understanding of public opinion regarding science and contested scientific issues-ultimately helping people to 'base their convictions upon evidence'. 


\section{Chapter 2: Rejection of Science}

What do I mean by rejection of science? In this chapter, I define rejection of science and provide five examples considered throughout the thesis. I also outline the negative consequences that rejection of science can have for individuals and society, providing an impetus for understanding the psychological drivers of such beliefs. I then explain why and how such an understanding can help to counter rejection of science, or even prevent its occurrence.

\subsection{DEFINING REJECTION OF SCIENCE}

Sometimes people hold beliefs that don't fit with the available scientific evidence. For example, they may believe climate change isn't happening, that vaccines cause autism, or that genetically modified (GM) crops are inherently more dangerous than non-GM crops. There are many ways these beliefs and attitudes are defined and labelled. For example, scholars have framed the phenomenon as 'science denial', 'scepticism', 'anti-science', 'contrarianism' or 'epistemically unwarranted beliefs' (Björnberg, Karlsson, Gilek, \& Hansson, 2017; Hansson, 2009, 2017; Rosenau, 2012). Within the wider academic literature, such beliefs are also conceptualized and measured in different ways, varyingly captured as risk perceptions, policy preferences, agreement with factual statements, intended or actual behaviours, and acceptability of certain types of research or technologies.

For the purposes of this thesis, I will adopt a clear definition and focus for my research, removing any ambiguity over what exactly it is that I am investigating. Here, I use the pragmatic terminology and approach of Lewandowsky, Oberauer and Gignac (2013). Lewandowsky and colleagues use the term rejection of science, defining this as "the dismissal of well-established scientific results for reasons that are not scientifically grounded" (p. 623). The authors also differentiate rejection of science from organised science denial (e.g. the public efforts of political elites and organisations to spread doubt and misinformation), stating that "our research on the rejection of science focuses on the factors that predispose people to be susceptible to organized denial" (p. 631). The term 'rejection of science' and the approach of identifying the individual differences associated with disbelief in scientific findings have been adopted by other psychology researchers (Hornsey \& Fielding, 2017; Rutjens, Sutton, \& van der Lee, 2017). Rejection of science in this context is 
typically operationalised as agreement or disagreement with statements that are supported by an established scientific consensus (or opposed in the case of reverse items).

Some clarification of the definition is required. Rejection of science refers to an individual holding a belief or position in opposition to the best available evidence. However, this might not constitute, from the individual's perspective, an active rejection of scientific evidence. Indeed, some misinformed contrarians may believe that 'the science is on their side' (Pasek, 2017). Thus, the rejection in question is not necessarily conscious.

\subsection{SCOPE OF THESIS}

This thesis reviews research into the psychological foundations of rejection of science and presents six original studies to further this body of work. It is impractical (if not impossible) to cover all research pertaining to public beliefs about scientific issues generally. This thesis will not extensively review the scientific evidence for each of the rejection of science issues covered in the following studies-I take the over-arching reports of peak scientific bodies such as the World Health Organisation and the US National Academies of Sciences as the strongest evidence of a scientific consensus. I acknowledge that this may change. In this regard, I adopt philosopher of science Sven Hansson's (2017) definition of science as representing the best available evidence at the time. All the scientific positions examined in this thesis have been considered in previous psychological research, though, to my knowledge, never simultaneously.

\subsection{EXAMPLES OF REJECTION OF SCIENCE}

This section outlines several examples of rejection of science, covering the five main science-relevant issues examined in this thesis. They are not intended to be comprehensive but provide a background primer for the next chapter and the studies that follow. They also give some indication of the prevalence of rejection of science in the New Zealand context, where most of the studies in this thesis are conducted.

\subsubsection{Climate change}

Climate change is perhaps the most well-studied issue where concerns have been raised over the gap between the views of the public and scientists. A strong scientific consensus supports the position that the release of greenhouse gases from human activities is causing the Earth's atmosphere to warm, and that this warming will have negative environmental, social and economic impacts (Cook et al., 2016; IPCC, 2014). Despite this robust scientific 
consensus, scepticism of the reality of climate change is relatively widespread in developed countries (Tranter \& Booth, 2015). This public denial has been supported, to some extent, by vested interests, such as fossil fuel companies, casting doubt on the consensus and spreading misinformation (Dunlap \& McCright, 2011; Oreskes \& Conway, 2010). While acknowledging the role of this misinformation in shaping public opinion, psychologists have also sought to identify the individual differences which lead some people and not others to deny the reality of climate change (Lewandowsky, Oberauer, et al., 2013).

Several studies have differentiated between outright denial that the Earth's atmosphere is warming (trend denial) and the argument that warming is not due to human activities (attribution denial), noting the latter has become the more common form of climate denial (Björnberg et al., 2017; Poortinga, Spence, Whitmarsh, Capstick, \& Pidgeon, 2011). Throughout this thesis I will focus on attribution denial (although measures used in the following studies do capture both trend and attribution denial).

New Zealand exhibits some of the highest rates of climate change denial among developed countries. Analysing 2010 data from 15 developed nations, Tranter and Booth (Tranter \& Booth, 2015) report that $15 \%$ of New Zealanders disagree that human-caused climate change is occurring, second only to Australia and Norway in terms of denial. In a very large $(N=8,889) 2014$ survey of New Zealanders, Milfont, Wilson and Sibley (2017) report that $13 \%$ of respondents disagreed that human-caused climate change is happening. A separate population-weighted survey undertaken the same year reported that $18.7 \%$ of New Zealanders disagree with the statement 'Most scientists agree that humans are causing climate change,' while $28.2 \%$ were unsure (Leining \& White, 2015). In sum, previous research suggests that somewhere between 10 and $20 \%$ of New Zealanders believe that human-caused climate change is not occurring.

Many scholars, politicians and environmental groups have expressed concern over climate change denial. Mitigating and adapting to the impacts of a warmer atmosphere requires behavioural change at the individual level as well as public support for policies to limit emissions and manage the predicted consequences of climate change (IPCC, 2014). People who do not believe that humans are causing climate change are generally less likely to act in ways that lower their personal contributions to greenhouse gas emissions, such as driving less or consuming less meat (Bord, O’Connor, \& Fisher, 2000; de Boer, Schösler, \& Boersema, 2013). They are also less likely to support local or national policies which aim to reduce emissions, such as carbon taxes (Hornsey, Harris, Bain, \& Fielding, 2016). Thus, 
understanding and changing beliefs about the reality of human-caused climate change is a key challenge for scientists and policymakers who seek to prevent and minimise the negative consequences of climate change (Clayton et al., 2015; van Lange, Joireman, \& Milinski, 2018).

\subsubsection{GM food}

Many people around the globe believe that food made from genetically modified plants and animals poses a greater risk to humans than conventional food (Hoban, 2004; Lang, 2016). This belief persists despite a scientific consensus that GM foods are no more harmful than non-GM foods. The US National Academies of Sciences, Medicines and Engineering (2016b) undertook an extensive review of all available published and unpublished data regarding the health effects of GM food. The authors found no evidence that GM food was more dangerous than conventional food. Comprehensive reviews focusing on both human and animal diets have also reported no difference (Delaney, Goodman, \& Ladics, 2018; Domingo, 2016; Nicolia, Manzo, Veronesi, \& Rosellini, 2014; Van Eenennaam \& Young, 2014).

I must note that some people oppose GM for other reasons. Individuals may oppose GM because they are worried about the environmental impact of modified crops or the rise of economic monopolies due to patenting laws on GM seed (Lang, 2016; Sjöberg, 2004). While these may be legitimate concerns, US research indicates that the most common reason for opposition to GM food is the perception that GM foods are dangerous to eat (Fernbach, Light, Scott, Inbar, \& Rozin, 2019).

Despite the current scientific consensus, the belief that GM food is dangerous is prominent in New Zealand. A 2013 random postal survey of New Zealanders reported that $42.2 \%$ agreed that 'GM poses a significant risk to the health and safety of humans' and only $12.7 \%$ of respondents agreed that 'GM products are safe for human consumption' (Chikazhe, 2015). Opposition to GM crops in New Zealand became pronounced in the early 2000s and is speculated to be driven by regulatory lapses and perceived conflict with 'clean and green' environmental values (Edwards, 2017).

Misperceptions around GM food can have negative impacts. New Zealand legislation currently prohibits the release of live genetically modified organisms into the wider environment and the importation of fresh GM fruit, vegetables or meat (Ministry for the Environment, n.d.). Some commentators have warned that this position limits New Zealand's 
competitiveness in the global agricultural market (Edwards, 2017; Fritsche, Poovaiah, MacRae, \& Thorlby, 2018). More broadly, proponents of GM crops argue that GM foods can be beneficial on a global level. Crops engineered for greater yield, pest resistance, drought tolerance, or nutritional value can increase wellbeing and food security in the face of a growing global population and a rapidly changing climate (Huesing et al., 2016; Zhang, Wohlhueter, \& Zhang, 2016). Agricultural scientists have warned that consumer opposition to GM food will limit the adoption and subsequent benefits of GM crops globally (Lucht, 2015).

\subsubsection{Vaccines}

Vaccines are considered to be one of the greatest public health initiatives ever created and have been credited with saving millions of lives, yet many people around the world people believe vaccines pose a dire threat to human health (Ozawa et al., 2017). Although concern over vaccination has existed for as long as vaccines themselves, fears were stoked in 1998 when Andrew Wakefield published a (now retracted) study in The Lancet suggesting the Measles, Mumps and Rubella (MMR) vaccine caused autism in some recipients (Poland \& Jacobson, 2011). Further unsupported beliefs about vaccines include claims that vaccines contain toxic amounts of aluminium or mercury, or 'overload' the immune system (Wessel, 2017).

Numerous large-scale studies have now established that there is no link between vaccines and autism and that severe side effects are incredibly rare (Institute of Medicine, 2012; Omer \& Yildirim, 2019). However, some people still believe that vaccines are dangerous and vehemently and actively oppose any form of vaccination. Although such 'antivaxxers' do receive a great deal of media coverage, milder concerns about the efficacy or timing of vaccines are more widespread. Termed 'vaccine hesitancy', this unease or ambivalence around vaccination may pose a larger problem by decreasing vaccination rates (Shapiro et al., 2018). The World Health Organisation listed vaccine hesitancy as one of the top ten threats to global health (WHO, 2019).

Childhood vaccination rates in New Zealand are typically high (>90\%), but may be dropping (Paynter, 2018). A 2019 outbreak of measles in New Zealand was attributed, in part, to anti-vaccine sentiment and dropping immunisation rates (L. Bennett, 2019; PetousisHarris, 2019). Surveys of New Zealanders indicate that most are supportive of vaccination. A survey of 1,500 individuals found that five percent had concerns about vaccine safety and 
five percent were unsure about vaccine safety (Meningitis Foundation Aotearoa New Zealand, 2013). In a survey of over 18,000 New Zealanders, Lee, Duck, and Sibley (2017) report that $68.5 \%$ were confident that "it is safe to vaccinate children following the standard New Zealand immunisation schedule," while $26 \%$ were 'sceptical' 1 and $5.5 \%$ expressed strong opposition.

The decision to not vaccinate oneself or one's children increases the risk of contracting a preventable and potentially fatal disease (Phadke, Bednarczyk, Salmon, \& Omer, 2016). Beyond the individual, the decision to not vaccinate can affect the wider community. Some people, such as young children and those with compromised immune systems, cannot be vaccinated. As the number of unvaccinated individuals in a population grows the likelihood of outbreaks occurring and spreading among unvaccinated individuals increases (Fine, Eames, \& Heymann, 2011). For example, approximately $95 \%$ of the New Zealand population must be immunised against measles to maintain 'herd immunity' and prevent recurrent outbreaks of the disease (Ministry of Health, 2018). Thus, countering misperceptions about vaccine safety is important not just for the health of individuals but also public health generally.

\subsubsection{Fluoridation}

Fluoride is a natural compound that protects teeth, makes them stronger and prevents tooth decay. In some areas where natural levels of fluoride in drinking water are low, fluoride is added to the water to improve dental health. Fluoride absorbed from drinking water strengthens tooth enamel and decreases the likelihood of dental cavities. A strong scientific consensus supports the position that fluoride added to water in this manner does not pose a health risk and prevents tooth decay, particularly in areas where poor dental hygiene is prevalent (Pizzo, Piscopo, Pizzo, \& Giuliana, 2007; Royal Society of New Zealand, 2014). A Government-commissioned report estimated that fluoridation of New Zealand's drinking water over 20 years would save \$1,578 million (NZD) in the reduced treatment of dental decay and result in between 8,800 and 13,700 quality-adjusted life-years gained (David \& Poynton, 2015).

Despite this scientific consensus and evidence, an active movement of anti-fluoride campaigners opposes water fluoridation, arguing the addition of fluoride poses a health risk.

\footnotetext{
${ }^{1}$ Giving a rating of between 3-5 in agreement to the statement on a scale of 1 (strongly disagree) to 7 (strongly agree).
} 
Their arguments include claims that drinking fluoridated water lowers children's IQ or increases the risk of bone cancer and heart disease. These claims are not supported by comprehensive reviews of the available scientific evidence (Broadbent, Wills, McMillan, Drummond, \& Whyman, 2015; Royal Society of New Zealand, 2014). While some fluoride opponents raise ethical concerns over 'mass medication' of the population, most of the arguments against fluoride appear to focus on perceived health risks and a lack of efficacy (Knox, Garner, Dyason, Pearson, \& Pit, 2017). On the fringes of the anti-fluoride movement, some opponents also claim water fluoridation is part of a government conspiracy to make citizens more docile, or a secret ploy of the aluminium and fertilizer industries to sell off waste fluoride by-products (Armfield, 2007).

Unlike the preceding three issues (climate change, GM and vaccination), fluoride opposition is less well-studied as an example of rejection of science. However, opposition to fluoridation exists in many developed countries, including New Zealand (Griffin, Shickle, \& Moran, 2008; Royal Society of New Zealand, 2014). For example, extensive anti-fluoride lobbying in New Zealand led to Hamilton City Council ending water fluoridation in 2013 (this decision was overturned in 2014; National Fluoridation Information Service, 2014). Recent New Zealand data on public attitudes towards fluoridation is limited, but a 2009 survey of almost 3,500 New Zealanders reports that $23.5 \%$ of respondents believed there were risks associated with adding fluoride to the water (45.8\% selected 'don't know') and $15.1 \%$ were opposed to water fluoridation (Whyman, Mahoney, \& Børsting, 2016).

Given the benefits of fluoridation, public health researchers in both in New Zealand and overseas have called for greater efforts to understand and counter misperceptions regarding its efficacy and safety (Allukian et al., 2018; N. Wilson \& Beaglehole, 2014).

\subsubsection{Evolution}

The theory of evolution posits that all species developed, over generations, into their current form through a process of natural selection. Broadly, organisms which are better suited to a given environment are more likely to survive, reproduce and pass on genes which confer this 'fitness' to the next generation. This process, coupled with random genetic mutations, leads to species changing and diverging over generations. Many lines of biological and palaeontological research support the theory of evolution and it is widely accepted among biologists (Dobzhansky, 1973; Gregory, 2009). However, rejection of the theory of evolution is widespread among the public. The main driver of this rejection is the conflict 
between religious and scientific explanations for the variety of life on Earth. A literal interpretation of religious scriptures, including the Bible, Koran, and Talmud, requires the creation of humans to have been the result of divine intervention and not through evolution from earlier primate species. While there is a clear connection between religious beliefs and beliefs about evolution it is important to note that within (and across) religious denominations there is still a wide range of views on the acceptability of the theory of evolution (Scott, 1997).

Rejection of the theory of evolution is globally prevalent, and varies dramatically across nations. Miller, Scott, and Okamoto (2006) surveyed 34 countries and found that in most a sizable minority did not accept the theory of evolution as true. In the US, $60 \%$ of those surveyed rejected the theory of evolution. However, more recent surveys indicate that this figure is now lower. The Pew Research Center reports that about 18\% of Americans believe life on Earth was created in its current form, noting that question framing has a significant effect on responses (Smith, Alper, Diamant, \& Schiller, 2019). In New Zealand (not one of the nations surveyed by Miller et al., 2006), a substantial minority of people also reject scientific accounts of evolution. A 2012 poll reported that $23 \%$ of New Zealanders believe in 'creationism', as opposed to 'pure evolution' (45\%) or 'theistic evolution'(26\%), while $6 \%$ are unsure (White, 2013).

Unlike the other rejection of science issues considered in this thesis, rejection of evolution does not have direct health or environmental impacts. However, it may still have negative consequences. Pigliucci, Baum, and McPeek (2006) warn that the rise of creationist beliefs, particularly in the context of science education, can undermine trust in science and lead to rejection of science in other domains:

At a time when evolutionary biology is increasingly important in medicine, agriculture, and environmental science, antagonism towards evolution has the potential to negatively impact the development of sound public policy about our economic and environmental well-being. More broadly, the divisiveness of the debate promotes distrust of scientists, reduces support for the funding of basic science, and undermines science education at all levels. (p. 1)

\subsection{IMPORTANCE OF INVESTIGATING REJECTION OF SCIENCE}

The implicit motivation of much previous research on rejection of science is to ultimately challenge and correct counter-scientific views that can have negative impacts on 
individuals and society (e.g., Hornsey, Harris, \& Fielding, 2018b). By understanding what motivates people to hold counter-scientific beliefs in the face of scientific evidence, scientists, health professionals, policymakers, and science communicators can better persuade individuals to accept the best available evidence (Hornsey \& Fielding, 2017). ${ }^{2}$ The normative argument for persuasion is clear when considering issues such as those described in this chapter. People often must make decisions about these issues which have an ultimate effect on the health of themselves, others, or the environment (for example, parents must choose whether to or not to vaccinate their children). On the role of science in such decisions, Dietz (2013) writes “.... ga good decision must be factually competent. The beliefs used in making decisions should accurately reflect our understanding of how the world works. Here, the role of science is obvious: Science is our best guide to developing factual understanding" (p. 14082).

Rejection of science not only has an impact on individuals' decisions, but it can also have far-reaching impacts on wider public opinion and national policy. Small groups holding a minority view can sway the views of the public (Moscovici \& Mugny, 1983). In turn, public opinion has a substantial impact on policymaking and a vocal minority challenging scientific findings can push policy-makers away from evidence-based options (Adams, 2013; Burstein, 2003). Thus, efforts to reduce rejection of science at the individual and small group level can influence national policy. Establishing a shared common ground based on scientific evidence will not end debate. Society will — and should — continue to debate how we best limit $\mathrm{CO}_{2}$ emissions, if the state should mandate childhood vaccinations, and if GM crops should be permitted in a country. These policy decisions rest on wider social, economic and ethical considerations (Pielke, 2007). However, productive debate can only progress if key participants, including the public, can agree on the scientific evidence before them (Gluckman, 2013).

Understanding the underlying drivers of rejection of science can go beyond 'convincing sceptics' and inform science policy at a higher level, avoiding polarising scientific controversies altogether. When considering research agendas, public health risks or the

\footnotetext{
${ }^{2}$ I do acknowledge there is a wider social and ethical discussion to be had here regarding the use of psychological research in changing individuals' beliefs and behaviour, and the implication that people should be 'convinced of the science'. Indeed, in arguing for the use of communication framing in convincing science sceptics, Nisbet and Mooney (2007) warn that "some readers may consider our proposals too Orwellian" (p. 56). Due to considerations of space I will not cover this here but direct the interested reader to the recent work in the ethics of science communication (John, 2018; Medvecky \& Leach, 2017) and influence (Sunstein, 2016).
} 
adoption of emerging technologies, policymakers should consider how interventions or outcomes will be received by the public, and how factors such as ideology may influence public opinion. A case study of this approach can be seen in the recent work of New Zealand's Biological Heritage Challenge (the Challenge), a government-funded, mission-led research programme which aims to protect New Zealand's biodiversity and improve biosecurity. The Challenge has set up a social science research programme to understand the social-psychological drivers of New Zealander's attitudes towards novel pest control technologies such as gene drives. This research will inform the broader research programme of the Challenge and avoid expending resources on researching technologies which will ultimately prove unacceptable to the wider public (Biological Heritage, 2018).

Finally, researching rejection of science can also contribute to psychological theory more broadly. While some frameworks discussed in this thesis focus specifically on scientific beliefs (such as the Gateway Belief Model: van der Linden, Leiserowitz, et al., 2015; and the Attitude Roots Model: Hornsey \& Fielding, 2017), others seek to explain human attitudes and behaviour more generally (e.g. Motivated Reasoning: Kunda, 1990; and the Dual Process Model of Ideology: Duckitt \& Sibley, 2016). The studies in this thesis will reinforce and expand on these science-specific and general theoretical frameworks.

\subsection{SUMMARY}

In this section, I have defined rejection of science for the purposes of this thesis and outlined five examples. These case studies show that many people hold beliefs inconsistent with current scientific consensus, and these beliefs can have negative consequences for human health and prosperity. Thus, there are public interest arguments for attempting to shift public opinion on these issues towards the scientific consensus. Psychological research can offer practical insights for policymakers, scientists and communicators seeking to combat rejection of science, as well as contributing to broader psychological theory. 


\section{Chapter 3: Why Do People Reject Science?}

This chapter reviews several approaches to explaining the rejection of science described in the previous chapter. Along the way, I will signpost where research in this thesis will address gaps or questions raised by the studies reviewed. I must note that the structure of this review doesn't fully align with the order of the following studies; some of the more commonly cited reasons for rejection of science are covered first but are only incorporated into later studies.

In this review I first examine the possibility that people hold counter-scientific beliefs simply due to a lack of knowledge or awareness. Here I introduce the Gateway Belief Model which places perceptions of scientific consensus as a causal driver of personal beliefs about scientific issues. I also review research indicating that general attitudes towards science and scientists influence our beliefs about specific scientific research. I then explore how deeper ideological beliefs and attitudes also influence our beliefs about scientific issues, drawing on the phenomenon of motivated reasoning and the Attitude Roots Model. Here I review research linking ideological factors, such as political ideology and religiosity, to rejection of science. I will also introduce the Dual Process Model as a potentially useful tool for exploring rejection of science. The chapter concludes with a summary of the research aims of this thesis. I reiterate several gaps in the literature and outline how they will be addressed by the studies in this thesis.

\subsection{IGNORANCE}

Perhaps the most parsimonious explanation for rejection of science is that people are simply ignorant. Scholars have considered the possibility that a lack of scientific knowledge on the part of the public is to blame for rejection of science. Research has also examined the possibility that knowledge of the scientific consensus, rather than specific 'facts', underpins acceptance of science.

\subsubsection{The Information Deficit Model}

An overall lack of awareness of scientific evidence may lead people to hold beliefs contrary to the best available evidence. This view is captured in the Information Deficit Model. As summarised by Suldovsky (2017): 
Conceptually, the deficit model rests on the assumption that a lack of knowledge or information leads to science-inconsistent attitudes and behaviors, and, thus, the solution to science-inconsistent attitudes and behaviors is to supply publics with information in the hopes that this newfound knowledge will result in science-consistent attitudes and behaviors. (p. 2)

Two slightly different assumptions are encompassed in the model and the literature referencing the Deficit Model does not always distinguish between them (Suldovsky, 2016). Firstly, the Deficit Model is used to describe the claim that a lack of general knowledge about science and scientific processes (termed science literacy) underpins rejection of science. In this scenario, the rise of climate change denial, anti-vaccination attitudes, and GM scepticism is a product of a general lack of science literacy on the part of the public. Secondly, the term Deficit Model is used more narrowly to refer to the approach of communicators to provide more information in the hope of shifting personal beliefs on scientific issues. This second take on the Deficit Model describes the strategy of providing specific scientific information to shift people's beliefs on (often controversial) scientific issues public. My focus here will be on the first interpretation of the model, as it is more relevant to understanding rejection of science across different scientific domains.

The Deficit Model has received a great deal of criticism as an oversimplification of the public's beliefs and attitudes regarding science (e.g. Simis, Madden, Cacciatore, \& Yeo, 2016). There is weak empirical support for the model when it comes to publicly debated scientific issues. For example, Allum and colleagues (2008) conducted a meta-analysis of 193 nationally representative surveys and found scientific knowledge was only a weak predictor of attitudes regarding a range of scientific issues, including nuclear power and genetic modification of food. Other large scale surveys have reported similar results, noting that scientific knowledge, in isolation, explains little variation in individuals' attitudes regarding publicly debated scientific issues (Drummond \& Fischhoff, 2017; Evans \& Durant, 1995). Evans and Durant (1995) suggest that general science knowledge may be a stronger predictor of attitudes about scientific issues which do not conflict with political or religious ideology.

Studies 5 and 6 and in this thesis examine the role of general scientific knowledge in rejection of science, in combination with a range of other factors. These studies will shed 
light on the kinds of issues where rejection of science can, in part, be attributed to a lack of knowledge, rather than other demographic or ideological factors.

\subsubsection{Awareness of consensus}

A narrower approach to understanding information deficits focuses on individual awareness not just of a statement of fact (e.g. 'humans cause climate change') but on the existence of scientific consensus (e.g. 'most scientists agree that humans cause climate change'). This approach assumes that individuals who believe that most scientists agree on a given claim are likely to align their beliefs with the scientific consensus. It also follows that people may reject scientific findings because they do not perceive a scientific consensus, that is, they believe the science is not 'settled', or they believe scientific consensus supports their position (e.g. most scientists agree that humans are not causing climate change).

There is a reasonable amount of support for this view based on cross-sectional research. For example, Hornsey et al.'s (2016) meta-analysis exploring correlates of climate change scepticism reports a moderate correlation between perceptions of scientific consensus and personal beliefs regarding climate change. However, correlation doesn't equal causation. Following from such cross-sectional studies social psychologists have investigated the causal relationship between perceptions of consensus and rejection of science.

\subsubsection{The Gateway Belief Model}

To address the question of causality regarding the link between perceptions of scientific consensus and personal beliefs, van der Linden and colleagues developed the Gateway Belief Model (GBM; van der Linden, Leiserowitz, et al., 2015). The GBM places perceptions of scientific consensus as a 'gateway belief' - a causal driver of personal beliefs.

In an experimental test of the GBM, van der Linden et al. (2015) report that exposure to a simple message-' $97 \%$ of climate scientists have concluded that human-caused climate change is happening'-increased participants' perception of a scientific consensus, and this increase was in turn associated with an increase in personal belief that humans are causing climate change. The authors also report that this shift in personal belief was associated with increased support for climate mitigation policies. The authors conclude that perceptions of scientific consensus causally influence beliefs about climate change.

In explaining why perceptions of consensus influence beliefs, van der Linden et al. (2015) suggest that perceptions of consensus act as a heuristic - a mental shortcut. In psychological models of persuasion such as the Heuristic Systematic Model (Chaiken, 
Liberman, \& Eagly, 1989) and the Elaboration Likelihood Model (Petty \& Cacioppo, 1986) people use heuristics to avoid cognitively effortful deliberation, helping them reach a conclusion or make a judgement efficiently. Two previously studied heuristics are captured in scientific consensus information. The first is the expert heuristic (experts can be trusted), in which information from an expert source is considered more persuasive than information from a non-expert source. The second is the consensus heuristic (consensus implies correctness) where an argument supported by a majority is more convincing than one supported by a minority (Kruglanski \& Stroebe, 2005).

A number of studies have now replicated the results of van der Linden et al. (2015), and expanded the model to include not just climate change but other contested scientific issues such as vaccination and GM food (Dixon, 2016; van der Linden, Clarke, \& Maibach, 2015; van der Linden, Leiserowitz, \& Maibach, 2019). However, some studies have also reported that interventions which aim to increase perceptions of scientific agreement on climate change have no overall impact on personal beliefs (Dixon \& Clarke, 2013; Dixon, Hmielowski, \& Ma, 2017; Ma, Dixon, \& Hmielowski, 2019). In light of such results, a number of researchers have called for further research examining the causal direction of the relationship between perceived consensus and scientific beliefs (Bolsen \& Druckman, 2018a; Kohl et al., 2016; National Academies of Sciences, Engineering, and Medicine, 2016a; Pasek, 2017). A report from the National Academies of Sciences, Engineering, and Medicine (2016a) also notes that most research on communicating scientific information has taken place in the US. The authors call for future research to examine how the efficacy of communication approaches differs across national contexts.

All research examining the GBM to date has focused on how increasing perceptions of scientific consensus can reduce rejection of science; no research has examined if decreasing perceptions of consensus has a symmetrical effect on scientific beliefs. This absence is surprising given the many well-documented examples of vested interests actively seeking to decrease perceptions of scientific consensus on publicly debated issues (Oreskes, 2015; Oreskes \& Conway, 2010). Previous research shows that exposure to climate sceptic arguments and conspiracy theories can reduce perceptions of scientific consensus regarding climate change (Cook, Lewandowsky, \& Ecker, 2017; van der Linden, 2015b; van der Linden, Leiserowitz, Rosenthal, \& Maibach, 2017). Would such decreases in perceived consensus lead to increased personal scepticism regarding the reality of human-caused climate change? 
Study 1 addresses this question alongside a replication of the original GBM experiment. This study investigates the impact of reading a message detailing a high or low level of scientific consensus on one of two issues (climate change or GM food). Do these messages change individuals' perceptions of a scientific consensus? And, if so, do these changes influence personal beliefs about the issue in question?

Experiments such as that of van der Linden et al. (2015) offer a strong test of causality. However, they take place in controlled settings over a short period with all other factors held equal and only test one possible causal direction. It is possible that individuals also draw on their own personal beliefs as a guide for estimating the level of scientific consensus in the absence of cues (Kahan, 2017). That is, peoples' estimates of scientific agreement may be biased by their own beliefs; they may guess that most scientists support their own view. Such a situation could be explained by unconscious self-deception (arising from a "desire to see the self and the world in way that favour the self"; Chance \& Norton, 2015, p. 104) such that people conclude that their views are supported by the authority of a scientific consensus (or are at least not contrary to one). Outside of immediate experimental settings, individuals may also be biased in the information that they seek out, assimilate and remember (Corner, Whitmarsh, \& Xenias, 2012; Hennes, Ruisch, Feygina, Monteiro, \& Jost, 2016). In this regard, people whose beliefs are at odds with the (objective) scientific consensus on, say, climate change, may develop a skewed perception of the level of scientific agreement regarding the reality of human-caused climate change. Thus, it is possible that personal beliefs influence perceptions of consensus, rather than vice versa.

However, it is not immediately clear how such an association might be experimentally tested. Longitudinal methods present an alternative approach to investigating the link between perceptions of scientific consensus and rejection of science. By examining consensus and climate change beliefs over time one can make causal inferences about the link between these variables 'in the wild' (Selig \& Little, 2012). If perceptions of scientific consensus causally drive belief in climate change outside the lab, we should expect to see such perceptions prospectively predict climate change belief at a later timepoint. Longitudinal analysis also allows examination of the reverse causal path: do personal beliefs predict later perceptions of consensus? Study 2 takes this longitudinal approach, analysing data collected from students at two time points to investigate the effect of perceptions of consensus on personal beliefs (and vice versa) over time. 


\subsection{GENERAL ATTITUDES TOWARDS SCIENCE}

Another possible explanation for rejection of science is a lack of trust in scientists or generally negative attitudes towards science. Evans and Durant (1995) report that the belief structure of general attitudes towards science is unidimensional; in a nationally representative UK survey the authors found that responses to a range of science-related items such as 'scientists can be trusted to make the right decisions' and 'science makes our way of life change too fast' all tap a single underlying evaluative factor. A positive general attitude towards science was a strong predictor of support for practical applications of science such as developing faster computers or more efficient powerplants. However, general evaluation of science and scientists was a poor predictor of support for what the authors describe as 'morally contentious' science, such as genetic engineering and the use of human embryos in research. They conclude that "measures of general attitudes are inadequate as a guide to what the public may think of specific areas or scientific research" (p. 70).

\subsubsection{Trust in scientists}

Overlapping with general evaluations of science, distrust of scientists as a group has also been examined as a potential explanation for rejection of science. Trust is a notoriously difficult concept to pin down (Irzik \& Kurtulmus, 2018; Nadelson et al., 2014). In the context of science, trust can be assumed to represent a perception of scientists as credible, likely to tell the truth, and sharing the interests of the public (National Academies of Sciences, Medicine, and Engineering, 2015). I would note that some conceptualisations of trust distinguish credibility, that is, expertise, from trustworthiness (Fiske \& Dupree, 2014). Put another way, some individuals may perceive a scientific source as an expert (i.e. they likely know the truth) but not trustworthy (i.e. they will not tell me the truth). Most measures of 'trust in scientists' capture both aspects (e.g. Chinn, Lane, \& Hart, 2018; Nadelson et al., 2014).

Trust in scientists is a weak to moderate predictor of beliefs about controversial scientific topics, with individuals who express greater trust in scientists generally more likely to align their views with those of scientists on issues such climate change (Hornsey et al., 2016), evolution (Nadelson \& Hardy, 2015), GM food safety (Marques, Critchley, \& Walshe, 2015) and vaccines (Sarathchandra, Navin, Largent, \& McCright, 2018).

Trust in scientists has been operationalised in a number of different forms and scales (Brossard \& Nisbet, 2006; Farias, Newheiser, Kahane, \& de Toledo, 2013; Nadelson et al., 
2014). Recently Hartman et al. (2017) developed a reliable short Credibility of Science scale (COSS), which measures "a base-level tendency to positively or negatively evaluate scientific methods, fields, authorities and the ideas they promote" ( $p$ 359), including "the extent to which one's default tendency is to trust in the methods and findings of science” (p. 358). Unlike other trust scales, the COSS was developed using Item Response Theory, and Hartman et al. report good discriminant and convergent validity. Lower scores on the scale (i.e. perceiving scientists as not credible or trustworthy ${ }^{3}$ ) predict rejection of science across a range of scientific issues, including climate change, GM food, and vaccination. However, in discussing their scale, Hartman et al. highlight the need for further research:

Next steps in advancing a more integrative research program should include the testing of structural models of scientific beliefs to better understand the unique contribution and path "location" of [perceived credibility of science] relative to other predictors of issue-specific science beliefs. For example, we might hypothesize that the COSS (partially) mediates the relationship between highly general dispositions or worldviews (e.g., conspiracist ideation, individualist ideology) and more issue-specific science beliefs. (p. 368)

Studies 5 and 6 in this thesis address this point, employing SEM to examine the extent to which the COSS measure explains variance in rejection of science, alongside other known predictors. These studies also investigate the mediating role of trust in scientists in explaining the link between distal ideological variables and rejection of science.

I must stress that trust (or lack thereof) alone does not fully account for the extent of public disagreement with the scientific consensus on issues such as GM food and evolution (Hartman et al., 2017). Researchers have sought to identify further factors, such as ideology, which could explain the persistence of rejection of science in modern society and inform the design of interventions to persuade entrenched rejectors of science (Hornsey \& Fielding, 2017; Rutjens et al., 2017). Before delving into the links between ideology and rejection of science I will first introduce the concept of motivated reasoning.

\footnotetext{
${ }^{3}$ Although Hartman et al. (2017) define their construct as the 'perceived credibility of science', they acknowledge that this measure captures elements of trust and one of the items explicitly mentions trust (People trust scientists a lot more than they should). Thus, for ease of reading I will occasionally refer to this measure as 'trust in science' or similar.
} 


\subsection{MOTIVATED REASONING}

Endorsing politically conservative values, such as valuing tradition and limited government intervention, appears to have little to do with the veracity of claims that the release of carbon dioxide into the atmosphere ultimately increases the amount of energy the atmosphere absorbs from the sun. Yet we find that political ideology is one of the strongest predictors of disagreement with such claims (Hornsey et al., 2016; Lewandowsky, Gignac, \& Oberauer, 2013). Why?

Motivated reasoning offers a broad account of how personal beliefs and values influence the interpretation of scientific evidence and ultimately beliefs about scientific issues: individuals may be motivated to disagree with the evidence on important scientific issues (Kunda, 1990). Motivation in this context refers to cognitive tasks; as Kunda (1990) puts it, this motivation is "any wish, desire, or preference that concerns the outcome of a given reasoning task" (p. 480). Kunda describes two forms of motivated reasoning. The first is accuracy motivated reasoning - driven by the desire to arrive at the most accurate conclusion. The second is directed motivated reasoning where thinking is directed towards the goal of arriving at the most desirable, but not necessarily accurate conclusion. In this form of motivated reasoning individuals are biased by 'what they want to be true'. This directional motivation can be explained in the context of Festinger's theory of cognitive dissonance (Kassarjian \& Cohen, 1965; Kunda, 1990). The holding of mutually incompatible beliefs creates a psychological discomfort which individuals seek to reduce through a variety of reasoning mechanisms. According to Druckman and McGrath (2019), individuals may selectively seek out information which supports their desired conclusion (confirmation bias or selective exposure), exhibit bias in their assessment of undesirable evidence (prior attitude effect) and more rigorously counter-argue and critique such evidence (disconfirmation bias).

Kunda's (1990) seminal article describing motivated reasoning specifically highlights several studies indicating that people engage in directionally motivated reasoning when interpreting scientific information. For example, smokers are less convinced by the findings of the US Surgeon General's report on the health risks of smoking, compared to non-smokers (Kassarjian \& Cohen, 1965). A similar study presented participants with a news report claiming that caffeine consumption is linked to fibrocystic disease in women (Kunda, 1987). Caffeine-drinking women expressed greater doubt about the conclusions of the report while non-caffeine-drinking women and men (regardless of caffeine intake) found the report believable. Based on these and other studies, Kunda (1990) concludes that “...the evaluation 
of scientific evidence may be biased by whether people want to believe its conclusions" (p. 490).

Kunda's (1990) examples focus on individual studies but examples of motivated reasoning can also be found regarding broader scientific claims. Campbell and Kay (2014) offer a motivated reasoning account of the connection between political ideology and rejection of climate science, arguing that the proposed solutions to climate change (regulations, carbon taxes) conflict with fundamental political values of conservatives. Thus, in the absence of alternative solutions, those on the political right are motivated to discount the evidence regarding climate change and maintain a climate sceptical viewpoint. In a similar vein, religious fundamentalists discount and disbelieve evidence for evolution because it poses a threat not only to their beliefs about the origin of humans but also to their wider religious worldview and identity (Langman, 2017). Motivated reasoning, in various forms, has also been invoked in explaining the rejection of science regarding vaccination and GM food (Blancke, Van Breusegem, De Jaeger, Braeckman, \& Van Montagu, 2015; Browne, 2018). It is important to note that motivated reasoning can explain the formation of counterscientific beliefs when evidence (or its implications) conflicts with values and ideology (as in the example of Kay and Campbell (2014) above ${ }^{4}$ ), but it also explains the persistence of such beliefs once formed. People assess new information in light of their prior beliefs and are more inclined to positively evaluate and accept evidence which reinforces those beliefs.

Druckman and McGrath (2019) warn that there has been substantial concept creep regarding the term motivated reasoning, with some researchers assuming that rejection of science is solely a product of motivated reasoning. The authors note that an overall lack of trust in scientific sources may lead an accuracy motivated individual to 'rationally' disregard scientific evidence in pursuit of reaching a true conclusion (see also Cook \& Lewandowsky, 2016). Druckman and McGrath write:

“... individuals may doubt scientific advice because they believe it is not motivated solely by truth, and will not lead to an accurate belief. This differs from a directionally motivated person who doubts scientific advice because the content of that advice contradicts an existing belief. In both cases, scientific authority is disregarded, but for different reasons" (p. 116).

\footnotetext{
${ }^{4}$ Sometimes referred to as 'value-protective cognition' (Druckman \& McGrath, 2019).
} 
Studies 5 and 6 in this thesis examine this point by incorporating a measure of trust in scientists (Hartman et al., 2017) into models examining the effect of ideological factors, such as conservatism, on rejection of science across a range of issues. This allows us to determine if people reject science due to ideological conflicts (suggesting a directional motivation), or instead due to a more generalised distrust of information from scientists (which is compatible with an accuracy motivation).

\subsubsection{The Attitude Roots Model}

Hornsey and Fielding (2017) incorporate motivated reasoning into their transtheoretical Attitude Roots Model. The authors present the model as a framework for understanding why some people hold counter-scientific beliefs that go against the best available evidence, aiming "to synthesize the motives that have received empirical attention... and to do so using a transtheoretical language that can help explain a wide range of "anti-enlightenment" beliefs" (p. 460). They distinguish 'surface attitudes', such as climate change denial or creationism, from 'attitude roots' which are "the underlying fears, ideologies, worldviews, and identity needs that sustain those attitudes" (p. 460). Hornsey and Fielding broadly categorise attitude roots into six themes: ideologies, values, and worldviews; conspiratorial ideation; vested interests; personal identity expression; social identity needs; and fears and phobias. The research in this thesis focuses on the first two (and arguably most studied) sets of 'attitude roots' - though I will return to the others in the final discussion.

The Attitudes Roots Model has explicitly informed recent studies examining climate change scepticism (Hornsey, Harris, \& Fielding, 2018a) and anti-vaccination beliefs (Hornsey et al., 2018b). A similar approach can be also seen in other psychological research investigating rejection of science across multiple issues (Lewandowsky, Gignac, et al., 2013; Rutjens et al., 2017). These survey-based studies highlight the importance of examining potential drivers of rejection of science simultaneously to avoid drawing conclusions based on spurious associations. For example, conservative political ideology and religiosity tend to be correlated (Jost, Kruglanski, et al., 2003), so any research examining the role of religiosity in rejection of science should also include political ideology and account for this overlap through statistical methods such as multiple regression (Rutjens et al., 2017). These studies and others have contributed to a growing body of knowledge regarding the circumstances under which ideologies, worldviews, and conspiracy thinking drive motivated rejection of science (Lewandowsky \& Oberauer, 2016; Rutjens, Heine, Sutton, \& van Harreveld, 2018). 


\subsubsection{Attitude roots: Ideological predictors of rejection of science}

This section briefly reviews how ideological factors relate to beliefs about certain debated scientific issues and science more generally. Here I employ a broad definition of ideology, in line with the Oxford English Dictionary definitions: 'A system of ideas and ideals' and 'The set of beliefs characteristic of a social group or individual' (see also, Converse, 2006; Knight, 2006). This deviates from the usage of the term to refer only to political ideology - which is just one of the ideological factors covered below. This review of the research is not intended to be exhaustive, but to give some indication of the extent to which different ideological factors are associated with rejection of science across different issues.

\section{Political ideology}

Political ideology can be defined as a broad set of interconnected beliefs relating to society and government, often presented as a unidimensional spectrum from 'liberal' to 'conservative' (but see Section 3.3.3 below). Individuals' placement on the liberalconservative spectrum reliably predicts their voting behaviour, as well as their support for real-world and hypothetical policy issues, and a number of non-political preferences such as music choice or interest in art (reviewed in Jost, Kruglanski, et al., 2003). Jost, Glasser, et al. (2003), argue that political conservatism (vs liberalism) represents, at its core, resistance to change and acceptance of inequality. They describe political conservatism as a form of motivated reasoning, which they term motivated social cognition. In this regard, people adopt conservative ideologies and beliefs as they fulfil a range of existential and epistemic needs relating to managing uncertainty and threat.

Political ideology and partisanship are also associated with rejection of science. This is most apparent in the case of climate change scepticism, where conservatives are more likely to reject the reality of human-caused climate change (McCright \& Dunlap, 2011). In a metaanalysis of 171 studies, Hornsey et al. (2016) report political conservatism to be a moderate, negative predictor of climate change acceptance. As noted earlier, conservative resistance to government regulations such as carbon taxes has been suggested as a key factor explaining the link between politics and climate change denial (Campbell \& Kay, 2014).

Political ideology is also associated, albeit less consistently, with rejection of science in other domains. Some survey-based studies report that conservatives are more likely to reject the mainstream scientific position on vaccination (Baumgaertner, Carlisle, \& Justwan, 2018; 
Blank \& Shaw, 2015; Rabinowitz, Latella, Stern, \& Jost, 2016) and the safety of GM food (McFadden, 2016). Conservatism is also a predictor of rejection of the theory of evolution, even after accounting for the effects of religiosity (Drummond \& Fischhoff, 2017). However, other studies report conflicting results, finding no link between conservatism and rejection of sciene on these issues. For example, McCoy's (2018) analysis of US Pew survey data reports a non-linear relationship between political ideology and vaccine beliefs, such that the most conservative and most liberal respondents were least likely to believe that vaccines are safe. On the issue of GM food safety, several studies have reported that political conservatism is unrelated to GM food beliefs (Drummond \& Fischhoff, 2017; Elder, Greene, \& Lizotte, 2018; Lewandowsky, Gignac, et al., 2013; Rutjens et al., 2017) or in fact predicts acceptance, rather than rejection, of the mainstream scientific position on GM food when other factors are taken into account (Biel \& Nilsson, 2005). Taken as a whole, there is strong evidence that political conservatism is a key driver of rejection of science regarding climate change, somewhat weaker evidence that conservatism is related to rejection of vaccine science and limited evidence that it is associated with views on GM food safety.

Conservatives also hold less positive attitudes about science and scientists generally. Self-reported conservatives (vs. liberals) report lower in trust in scientists (Gauchat, 2012), perceive scientists to be less credible (Hartman et al., 2017), and have less faith and confidence in science (P. N. Cohen, 2018; Rutjens et al., 2017). In the US, it appears that this political divide has increased over the last four decades (P. N. Cohen, 2018; Gauchat, 2012).

The apparent anti-science bent of conservatives has led some scholars and commenters to declare that the right-wing of politics is inherently 'anti-science' (Mooney, 2012). Jost (2017) suggests that conservatives may be more cognitively inclined towards directionally motivated reasoning, as they exhibit lower levels of cognitive reflection and higher levels of self-deception. This 'asymmetry thesis' is subject to ongoing debate (J. Baron \& Jost, 2019; Ditto, Clark, et al., 2019). Opponents of this position point out that liberals are just as likely to exhibit motivated reasoning in controlled laboratory experiments (Ditto, Liu, et al., 2019). That is, liberals are just as biased as conservatives when it comes to assessing politically unpalatable evidence and updating their beliefs. Lewandowsky and Oberauer (2016) speculate that the trend of conservatives being more likely to reject science may be a quirk of historical and political context, "in which publicly contested scientific findings primarily happen to challenge the worldviews of conservatives rather than liberals" (p. 220). The authors add that, based on the available evidence, firm conclusions cannot be drawn 
regarding the psychological motivation of conservative's rejection of science (see also Lewandowsky, Cook, \& Ecker, 2017).

Notably, virtually all the research examining political ideology as a driver of rejection of science is based on self-report survey data (e.g., Lewandowsky, Gignac, et al., 2013; Rutjens et al., 2017). In Study 3 I examine the link between politics and science from a different perspective, using 'following' behaviour on the social media platform Twitter. Here I explore the relationship between following New Zealand conservative (vs liberal) political accounts and following accounts rejecting the scientific consensus on climate change, GM food, and vaccination. The use of social media data offers a novel approach to examining rejection of science, using behavioural measures to investigate associations observed in studies using self-report measures.

\section{Free market ideology}

Another ideological factor associated with rejection of science on some issues is free market ideology - a commitment to the principles of a free market with minimal government regulation and intervention (Heath \& Gifford, 2006). Free market beliefs are often a component of broader political conservatism, and in some cases free market ideology has been used as a proxy for general political conservatism (e.g., Cook et al., 2017). Support for free markets reliably predicts rejection of climate change science (Heath \& Gifford, 2006; Hornsey et al., 2016). There is limited evidence that free market beliefs are also associated with rejection of science in other domains. Lewandowsky, Oberauer, et al. (2013) report that, in an online community sample, free market beliefs predict rejection of climate science but also predict rejection of science on other, unrelated claims (smoking causing cancer and HIV causing AIDS). Lewandowsky, Gignac, et al. (2013) also examined the unique effects of political conservatism, free market ideology and conspiracy ideation on scientific beliefs in a representative US sample. They report that free market beliefs predict climate change and vaccine scepticism (but not GM food opposition). The authors suggest the link between free market support and vaccine scepticism arises from opposition to government promotion of mandatory vaccination. Notably conservatism and free market ideology, though highly correlated, had opposing effects on vaccine scepticism in Lewandowsky, Gignac, et al.'s model; unlike free market endorsement, political conservatism in fact weakly predicted vaccine support. This highlights the need to consider these constructs independently when modelling rejection of science. 


\section{Religiosity}

Religion is an ideological driver of rejection of science in some domains. This is most clear in contexts where religion and science offer competing explanations of the world. For example, a literal interpretation of the Bible or Koran requires the Earth to only be several thousand years old, and for all extant species -including humans-- to have been created in situ by a supreme being. Scientific research offers very strong evidence that this is not the case, with many lines of geological and paleontological evidence converging on the conclusion that the Earth is billions of years old, and life on Earth has evolved over millions of years to reach its current form (Dobzhansky, 1973). Unsurprisingly, given the epistemological conflicts described above, religious individuals are more likely to disagree with the theory of evolution, as well as the big bang theory and plate tectonics (Drummond \& Fischhoff, 2017; Pasek, 2017). These scientific positions clearly conflict with a literal interpretation of religious texts.

Religiosity predicts rejection of science in other domains, although results are inconsistent. As noted above, across virtually all cultural contexts, political conservatism and religiosity are correlated, therefore it is important to examine these ideological factors simultaneously to disentangle their independent effects on a given dependent variable (Rutjens et al., 2017). Religious individuals, particularly evangelical Christians, are less likely to agree that humans are causing climate change, even after controlling for political ideology (Ecklund \& Scheitle, 2018; Ecklund, Scheitle, Peifer, \& Bolger, 2017; McCright \& Dunlap, 2011).

Religiosity may be associated with rejection of science regarding GM food safety. Although there is little explicit condemnation of GM by the major monotheistic religions (Omobowale, Singer, \& Daar, 2009), much of the rhetoric around GM takes on quasireligious language - for example, accusations of scientists 'playing God' and 'tampering with the natural order' (Lull \& Scheufele, 2017). Larson (2018) reviews a number of US studies reporting a relatively consistent negative association between religious affiliation or strength of religious faith and positive evaluations of GM food. However, opposition to GM food itself does not necessarily constitute rejection of science. One might oppose GM food on religious grounds but accept the scientific consensus that GM food is not inherently harmful 
to humans. ${ }^{5}$ Yet through a process of motivated reasoning, religious adherents opposed to GM food may 'latch onto' unfounded arguments that GM food is inherently dangerous - the conclusion they 'want to be true'. Biel and Nilsson (2005) report that Swedish Christians (compared to atheists) are more likely to perceive GM food as threatening or dangerous. Not all studies have shown religiosity to be linked with GM food beliefs; some surveys in the US and Europe report no association between religious identification and perception of GM food as dangerous (Costa-Font, Gil, \& Traill, 2008; Funk \& Rainie, 2015). While there are some arguments for why religious individuals might be more inclined to reject the scientific consensus on GM food safety, empirical research to date does not offer conclusive evidence.

Religious beliefs may also be associated with negative attitudes towards vaccination, despite the fact that few religious doctrines prohibit vaccination (Grabenstein, 2013). In a meta-analysis of global research examining social predictors of vaccine hesitancy covering 76 studies, Larson et al. (2016) report an overall effect of religious affiliation, such that religious individuals are more likely express vaccine hesitancy. However, this study only examined bivariate associations; it did not control for potential confounding variables such as age, gender and political views. Some studies report no association between religious factors and vaccine beliefs. For example, in a survey of Australians, Browne et al. (2015) report no relationship between vaccine endorsement and religious affiliation or strength of religious faith.

There are some explanations as to why more religious people may be likely to reject science, even when it does not conflict directly with doctrine. Religious individuals may feel more uncomfortable about developments in modern science generally, perceiving scientists to be 'playing God', or viewing scientists as lacking in morality (Rutjens \& Heine, 2016). Religious individuals may also find that their interaction with science and scientific information is limited by their communities (Sherkat, 2010), or develop negative attitudes towards science as a result of discrimination from within the scientific community (Scheitle \& Ecklund, 2018). Indeed, across a series of surveys McPhetres and Zuckerman (2018) report that religiosity is associated with less interest in science and the belief that science is not important. They also report that these associations are explained, in part, by lower levels of science literacy and less trust in scientific sources among religious individuals.

\footnotetext{
${ }^{5}$ A parallel can be drawn with stem cell research. Many religious individuals oppose stem cell research on moral grounds, but do not disagree with the claims of scientists regarding its potential uses (Allum et al., 2017).
} 


\section{Conspiracy mentality}

Individuals who believe in one conspiracy theory are more likely to agree with other, seemingly unrelated conspiracy theories or even contradictory theories or fictitious conspiracies devised by researchers (Swami et al., 2011; Wood, Douglas, \& Sutton, 2012). Bruder et al. (2013) argue that this conspiracy mentality can be understood as a generalised political attitude, "a stable ideological belief system that allows predicting attitudes to specific attitude objects" (p. 26). This propensity to believe conspiracy-based explanations for phenomena has been linked to rejection of science across a range of issues. Indeed, opponents of the scientific consensus often invoke conspiracy theories as an explanation of why the evidence appears stacked against them (Goertzel, 2010). For example, a common theory put forward by climate change sceptics is that climate change is a hoax concocted by scientists to gain more research funding (Joseph E. Uscinski, Douglas, \& Lewandowsky, 2017). Conspiracy theories have also been espoused by opponents of vaccination, GM food, and fluoridation (Goertzel, 2010; Helmi, Kate Spinella, \& Seymour, 2018; Shapiro, Holding, Perez, Amsel, \& Rosberger, 2016).

Fitting with these observations, survey-based research shows that a propensity to believe in conspiracy theories is linked to rejection of science. Indeed, conspiracist mentality appears to be one of the more consistent psychological predictors of rejection of evidence across scientific domains. Lobato et al. (2014) report that individuals who scored higher on a conspiracy belief scale were also more likely to endorse 'anti-science' beliefs based on a scale measuring agreement with pseudoscientific (e.g. homeopathy) and rejection of science (e.g. climate change denial) statements. In a separate study Lobato and Zimmerman (2019) report that conspiracy mentality correlates with rejection of climate and evolution science but not science regarding GM food or vaccine safety. As noted above Lewandowsky, Gignac, et al. (2013) constructed a model predicting rejection of science regarding three issues: climate change, vaccination, and GM food. The authors report that conspiracy ideation (controlling for free market beliefs and conservatism) is the only consistent predictor of rejection of science across all three issues. In a separate study, the same authors also report conspiracy ideation predicts disagreement with the mainstream scientific position on two further medical issues: smoking causing cancer and HIV causing AIDS (Lewandowsky, Oberauer, et al., 2013).

Conspiracy mentality is also associated with a negative view of science generally. Hartman et al. (2017) report that conspiracy ideation is negatively correlated with the 
perception of scientists as trustworthy and credible. As an 'attitude root' (Hornsey \& Fielding, 2017) of rejection of science, conspiracy thinking is a difficult driver to overcome in efforts to inform audiences. The most extreme conspiracy adherents argue that any evidence contrary to their position is evidence of the conspiracy itself. This circular logic can lead to ever more byzantine accounts that allow conspiracy beliefs to continue in the face of conflicting evidence (Lewandowsky, Cook, \& Lloyd, 2018).

\section{Section summary}

The key takeaway of the research reviewed above is that different social and ideological factors predict rejection of science for different issues. Reviewing such research and several original studies, Rutjens and colleagues (2017) drew the very reasonable conclusion that "different forms of science acceptance and rejection have different ideological roots..." (p. 396). While previous studies have examined the role of these predictors across scientific issues, none have considered the four reviewed above simultaneously. For example, Lewandowsky, Gignac, et al. (2013) include conservatism, free market beliefs, and conspiracy thinking — but not religiosity — as predictors of scepticism regarding climate change, vaccines, and GM food. Similarly, Rutjens et al. (2017), examining the same dependent variables, include religiosity and political ideology as predictors but omit conspiracy thinking and free market ideology. Given the overlap between these various constructs, such omissions invite the possibility of misidentifying significant antecedents of rejection of science.

In Studies 5 and 6, I examine the unique effects of ideological factors on rejection of science across several domains, in US and NZ samples respectively. Here the ideological attitude roots reviewed above (political conservatism, free market ideology, conspiracy mentality, and religiosity) are analysed in concert with two with further potential predictors of rejection of science (scientific knowledge, trust in scientists). By examining these predictors simultaneously, I can determine the relative contributions of each in explaining rejection of science across different issues. However, these studies also go one further step in attempting to understand the origins of rejection of science, seeking to answer the question: what drives the drivers of rejection of science? In the next section, I draw on several lines of empirical and theoretical evidence to argue that the four attitudes roots reviewed aboveconservatism, free market ideology, religion and conspiracy thinking - and attitudes towards scientists share a deeper common substrate in beliefs about authority and inequality. 


\subsubsection{The Dual Process Model: RWA and SDO as distal drivers of rejection of science}

The construct of political ideology has often been assumed to be unidimensional, with individuals landing somewhere on a spectrum from left to right or liberal to conservative ( $\mathrm{S}$. Feldman \& Johnston, 2014). However, psychologists have posited that there are two dimensions underlying political ideology-two separate, but related drivers of conservative or liberal ideology (reviewed in Duckitt \& Sibley, 2009). The Dual Process Model of Ideology (DPM; Duckitt \& Sibley, 2009) is one of the most robust and well-researched models which captures this notion.

The DPM draws together two distinct and rich lines of psychological inquiry. The first is Altemeyer's work developing and refining the construct and measurement of Right-Wing Authoritarianism (RWA), building on the early work of Theodore Adorno and colleagues seeking to measure the 'authoritarian personality' (Altemeyer, 1996, 1998, 2006). Altemeyer describes RWA as three covarying attitudinal clusters: authoritarian submission, authoritarian aggression, and conventionalism. Submission refers to a tendency to submit to perceived legitimate authorities in society (e.g. police, religious officials). Aggression refers to the willingness to cause harm (of any kind) to others in the name of perceived authorities, particularly individuals or groups who threaten the social order or norms. Conventionalism refers to the acceptance and commitment to traditional social norms, often those linked with Judaeo-Christian religions, and resistance to change. Across many studies, Altemyer (1996) presents results indicating that questionnaire items covering these three attitudinal clusters can be combined into a single scale capturing a broad unidimensional construct: RWA. The second pillar of research upon which the DPM rests is Sidanus and Pratto's (1999) Social Dominance Theory. This body of work seeks to describe the institutional and individual factors which maintain group-based hierarchies in human society. Of key importance to the theory is the construct of Social Dominance Orientation (SDO), an individual difference variable capturing "the degree to which individuals desire and support group-based hierarchy the domination of 'inferior' groups by 'superior' groups”' (Sidanius \& Pratto, 1999, p. 48).

In the context of the DPM, Duckitt and Sibley (2016) describe RWA and SDO as "broad motivationally (or value) based social attitude or ideological dimensions" (p. 212). Specifically, the authors describe RWA as "a threat-driven attitudinal expression of the values or motivational goals of collective security, control, stability, and order.” And SDO as "a competition-driven attitudinal expression of the values or motivational goals of power, dominance, and superiority" (p. 212). These ideological attitudes explain substantial variance 
in people's prejudice towards certain groups and support for right-wing and ethnocentric policies. As Duckitt (2001) explains, “These dimensions seem to powerfully structure social, political, and intergroup behavior" (p. 49).

The DPM also theorises that RWA and SDO are in turn driven by a combination of personality traits, value orientations, social worldviews, and situational factors. In terms of psychological factors, RWA is associated with specific personality traits (low Openness to Experience and high Conscientiousness) and Schwartz (1992) value orientations (Security, Conformity, and Tradition vs Stimulation and Self-Direction). SDO is related to a different set of personality traits (low Agreeableness) and values (Achievement, Power, and Hedonism vs Universalism, Benevolence). In terms of worldviews, the DPM posits that RWA is underpinned by the perception of the world as a dangerous and threatening place while SDO is driven by the perception of the world as a competitive dog-eat-dog jungle. These worldviews may be socialised in early life by growing up in threatening or competitive environments, respectively (Duckitt \& Sibley, 2009). Figure 3.1 gives an overview of the DPM, outlining the antecedents and consequences of RWA and SDO.

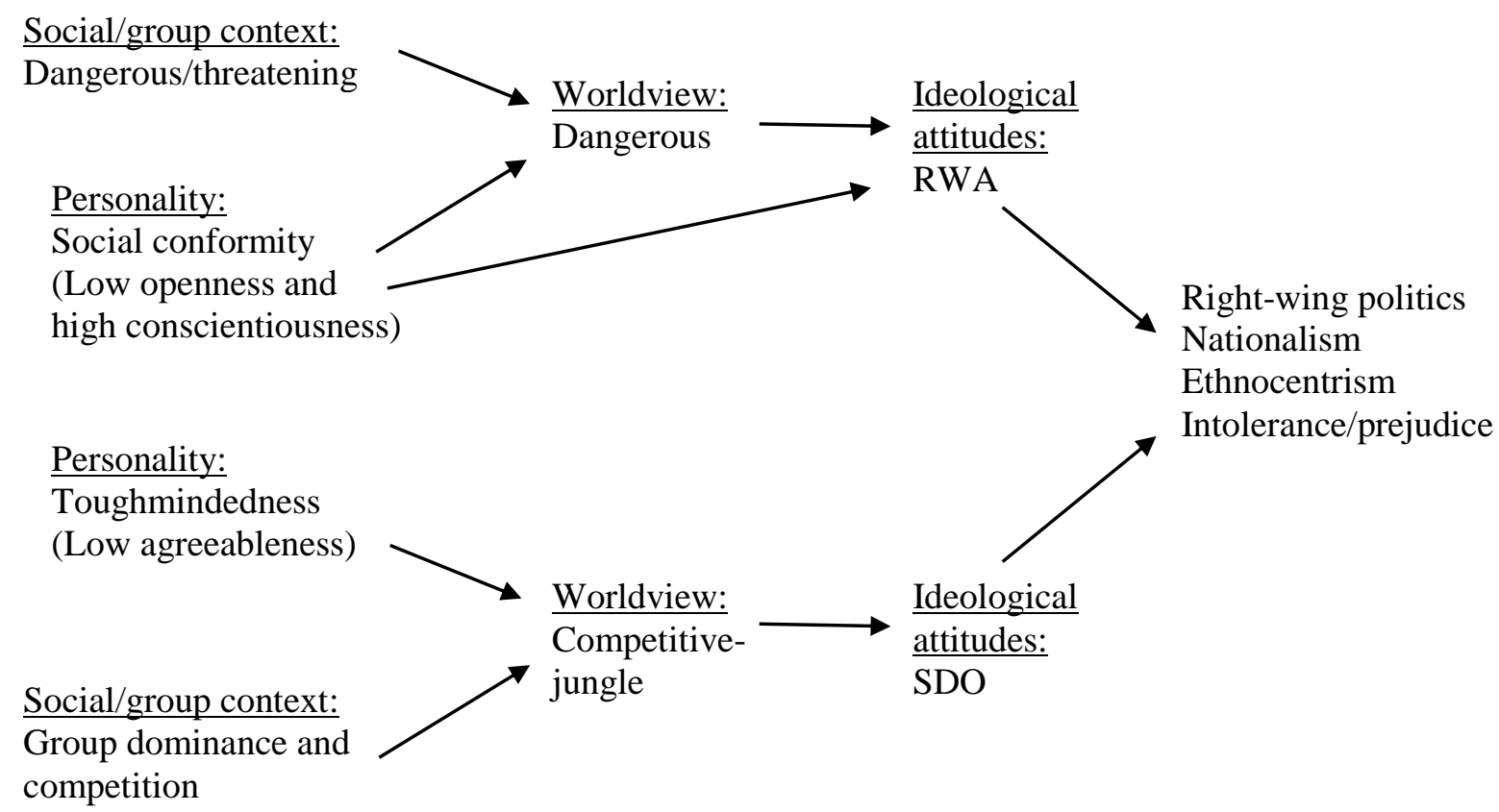

Figure 3.1. The Dual Process Model of Ideology. Adapted from Duckitt and Sibley (2009). 
The DPM has primarily been used in the study of intergroup prejudice, but researchers have also applied the model to a range of other socio-political attitudes, including support for the war in Iraq, economic policies, dietary preferences, political ideology and partisanship (Harnish, Bridges, \& Gump, 2018; Judge \& Wilson, 2019; Mcfarland, 2005; Sibley \& Wilson, 2007; M. S. Wilson \& Sibley, 2013). Wilson (2011) went as far as to say that when SDO is combined with RWA "it becomes a part of something that is as close to a grand theory of social behaviour as psychological researchers have ever had" (p. 7).

Given the association between political ideology and climate change, and the role of RWA and SDO as psychological antecedents of political ideology in the DPM, it is unsurprising that psychologists have also explored the direct links between these ideological attitudes and rejection of climate science. For example, Milfont et al. (2013) examined the relationships between RWA, SDO, and belief in human-caused climate change in a large $(N=$ 5,744) national New Zealand sample. The authors report that both RWA and SDO are significant predictors of climate change denial. A recent meta-analysis reinforces these findings. Stanley and Wilson (2019) meta-analysed the regression coefficients of RWA and SDO in predicting climate change denial across nine studies, finding that both ideological attitudes explained unique variance in belief in climate change.

No studies have examined the relationship between ideological attitudes RWA and SDO and rejection of science in other domains such as vaccination or GM food. However, I will make the case here that these constructs should be associated with counter-scientific beliefs for two reasons. Firstly, previous research indicates RWA and SDO are psychological antecedents of several of the ideological attitude roots described above. Secondly, RWA and SDO may also drive a general distrust or negative evaluation of science. I will elaborate on both these arguments in the following sections.

\section{$R W A$ and SDO predict predictors of rejection of science}

Empirical research and theory place RWA and SDO as psychological antecedents of the ideological roots of rejection of science reviewed in this chapter thus far: conservative political ideology, free market endorsement, conspiracy ideation and, arguably, religiosity.

In the case of political ideology, the link between ideological attitudes and political conservatism is well established. RWA and SDO predict unique variance in (and interact to predict) peoples' self-placement on a liberal-conservative spectrum and support for conservative political parties and policies (Wilson \& Sibley, 2013). Longitudinal and 
experimental research suggests that ideological attitudes influence political conservatism, rather than vice versa (Cantal, 2015; Matthews, Levin, \& Sidanius, 2009). This evidence supports the DPM claim that people high in either RWA or SDO will support conservative politics and parties, but for different reasons:

Persons high in RWA should prefer right-wing parties that emphasize law and order and defend traditional and religious values. Persons high in SDO should prefer rightwing parties that emphasize free market capitalism and antiwelfare policies. When right-wing parties espouse both sets of policies, RWA and SDO should predict their support similarly. (Duckitt \& Sibley, 2009, p. 105)

In many Western countries, the social and economic aspects described above have become intertwined by political parties aligning with a broader left-right or liberalconservative spectrum (Duriez, Van Hiel, \& Kossowska, 2005). Thus, we find that both high RWA and high SDO individuals self-identify as conservative in such political environments.

Psychologists also theorise that ideological attitudes underpin free market ideology specifically, though there is less empirical research investigating this link. The limited studies available indicate that both RWA and SDO predict free market endorsement (Buckland, 2014; Jost, Blount, Pfeffer, \& Hunyady, 2003; Roney, 2009; Sidanius \& Pratto, 1993). Why are people who are more authoritarian or accepting of inequality more likely to show an ideological commitment to free market principles? In the case of SDO, Social Dominance Theory and the DPM describe the belief that the free market is 'fair' as a legitimising myth that justifies unequal distribution of resources and status in society (Duckitt \& Sibley, 2016; Jost, Blount, et al., 2003; Sidanius \& Pratto, 1999). High SDO individuals endorse such myths because they protect the established hierarchies in society. ${ }^{6}$ The role of RWA in driving free market beliefs is less clear. Duckitt and Sibley (2016) and Feldman and Johnston (2014) suggest that free market endorsement is a product of anti-egalitarian, but not authoritarian attitudes. However, Azevedo, Jost, Rothmund, and Sterling (2019) rebut this argument, highlighting the 'resistance to change' aspect of RWA as a psychological driver of support for existing laissez-faire capitalism — and neoliberalism more generally—in modern economies. Thus, high SDOs endorse the free market as a just and fair system as it allows

\footnotetext{
${ }^{6}$ My use of the term 'high' here is relative; most people score well below the midpoint on measures of SDO and RWA. My discussion of people 'high in SDO', or 'high SDOs' should be taken to mean 'people who score above average on these scales'. As is common in the literature, I also will occasionally use terms like 'socially dominant' (high SDO) and 'authoritarian' (high RWA) as a shorthand (e.g., Altemeyer, 1998; Mcfarland, 2005)
} 
existing hierarchies to persist, while high RWAs may support the free market as the status quo, fulfilling needs for stability.

RWA and SDO predict belief in specific conspiracy theories as well as a more general acceptance of the existence of conspiracies (Bruder et al., 2013; Swami, 2012; M. S. Wilson \& Rose, 2014; Wood \& Gray, 2019). van Prooijen and Douglas (2018) summarise the extant research succinctly: "People who are dispositionally likely to perceive their ingroup as superior or to perceive outgroups as threatening display increased belief in conspiracy theories" (p. 902). Grzesiak-Feldman (2015) notes that the association between ideological attitudes and conspiracy belief may depend on the nature and alleged perpetrators of conspiracies. She suggests that high RWAs may be attracted to conspiracies that demonize deviant out-groups, but adds that this could evolve into more generalised conspiracy thinking, concluding: "RWA should be considered as one of the psychological antecedents of conspiracy theorizing, which may draw people towards a wide range of conspiracy theories" (p. 115). High SDO individuals may also be drawn to conspiracy theorising as a way to denigrate out-groups perceived as competing for resources or threatening established hierarchies. Grzesiak-Feldman (2012; cited in Grzesiak-Feldman, 2015) reports that Polish participants who scored higher in SDO were more likely to ascribe conspiracy stereotypes to Germans and Jews, two groups that might be considered powerful, competing out-groups (Dębski \& Niemczak, 2014; Golec de Zavala \& Cichocka, 2012).

RWA is consistently linked with religious identity and practices, strength of religious beliefs, and religious fundamentalism (Altemeyer, 1996; Altemeyer \& Hunsberger, 1992; Sibley, Robertson, \& Wilson, 2006). While this has been most commonly documented in largely Christian samples, Hunsberger (1996) reports RWA also strongly correlates with religious fundamentalism in Hindu, Muslim, and Jewish samples. The causal nature of the relationship between RWA and religiosity generally is unclear, and complicated by some conceptual overlap between the RWA scale and measures of religiosity (Mavor, Louis, \& Laythe, 2011). Hunsberger (1996) suggests RWA and religiosity, "shape and reinforce each other" (p. 41). On the one hand, authoritarians, "being relatively submissive to parents and other established authorities such as the clergy, tend to accept the religion of their youth more strongly than others and remain the "true believers' into adulthood" (p. 41). On the other hand, religious practice and teaching may also foster authoritarian attitudes around submission and punishment of dissent. In contrast to RWA, SDO appears to be unrelated, or weakly negatively related to religious belief (Altemeyer, 1998, 2004; Heaven \& Connors, 
2001; Sibley et al., 2006). Dallago et al. (2008) speculate that any negative relationship between SDO and religiosity may be due to the perception of religions as hierarchyattenuating movements (see also, Roccato, 2008).

To summarise, RWA and SDO are theoretically and empirically linked to several ideological antecedents — attitude roots — of rejection of science: political conservatism, free market ideology, conspiracy mentality, and, religiosity (RWA only). Thus, we can expect that RWA and SDO should be indirectly associated with rejection of science in instances where these attitude roots predict rejection of science. Studies 4, 5 and 6 test this hypothesis, exploring how factors such as political ideology and religiosity mediate the effects of RWA and SDO on rejection of science.

\section{RWA, SDO and broader attitudes towards science}

I have thus far reviewed how RWA and SDO may be linked to rejection of science in specific domains by several ideological variables or, in the words of Hornsey and Fielding (2017), attitude roots. However, the ideological attitudes RWA and SDO may also predict negative attitudes towards science and scientists more generally. In this section, I review the scant research in this area and offer some further theoretical justification for why high SDO and RWA individuals may be more denigrating or distrusting of science generally.

No studies have directly examined the relationship between RWA and evaluations of science or trust in scientists. However, several studies offer some indirect evidence. Theodore Adorno's early work in conceptualising authoritarianism incorporated a 'superstition and stereotypy' component in the development of the Fascism scale (F-scale) intended to measure 'prefascist tendencies'. As such, items in the F-Scale directly tapped pseudoscientific thinking and doubt in science, for example: 'Science has its place, but there are many important things that can never possibly be understood by the human mind' and 'Someday it will probably be shown that astrology can explain a lot of things' (Adorno, FrenkleBrunswik, Levinson, \& Sanford, 1950, p. 235). These items appear to intercorrelate with the other items comprising the F-Scale, which includes items that have been used in more contemporary measures of authoritarianism (Altemeyer, 1988). ${ }^{7}$ In explaining why people with authoritarian tendencies would eschew science, Adorno and colleagues draw on

\footnotetext{
7 The authors do not report item-total correlations for the F-scale items, but provide Discriminatory Power values, indicating the difference in mean scale score between the item 's top and bottom quartiles (Adorno et al., 1950, p. 260). These suggest that how people respond to the individual items noted here is related to their overall F-scale score.
} 
Freudian psychoanalytic theory, suggesting that a weakness of the ego among authoritarian individuals leads otherwise intelligent people to "resort to primitive, oversimplified explanations of human events" (p. 236). The F-scale has been roundly criticised for its poor psychometric properties and theoretical development. Martin (2001) describes it as "probably the most deeply flawed work of prominence in political psychology" (p. 1). Problems include the use of only positively worded items, allowing acquiescence bias to influence responding, and the application of Freudian theory which "legitimated circular interpretations" (p. 1). Acknowledging such criticisms, the documented relationship between a general scepticism of science and aspects of authoritarianism is still worth noting here.

Altemeyer's (1988) resurrection of authoritarianism research carried over several aspects of the F-Scale in the development of the RWA scale but dropped the 'superstition and stereotypy' cluster of items. However, his later research offers some hints about the relationship between RWA and attitudes towards science. Altemeyer (1996) briefly reports one study in which parents and students completed a measure of RWA and were also asked 'What is the nature of your most important outlook?' Among the nine possible responses was 'A scientific outlook. Science gives me my most basic understanding of things'. Altemeyer does not report full results but notes that respondents who selected this option had some of the lowest RWA scores in the sample (p. 213).

What about SDO? As with RWA, there is no research directly examining a link between SDO and general attitudes towards science. Pratto et al. (1997) come close, examining the extent to which SDO predicts students' preferences for a range of careers, including 'science'. The authors classed science as a middling career, neither hierarchyenhancing nor hierarchy-attenuating. They did not provide a justification, nor did they report on the specific association between SDO and preference for science as a career path (p. 41). We can, however, draw some inferences from looking at constructs analogous to SDO. Within the 'Cultural Cognition' framework, Kahan (2012a, 2012b) employs a measure of a hierarchy-endorsing cultural worldview drawn from Douglas and Wildavsky's (1982) Cultural Theory: the Hierarchy-Egalitarianism scale. This scale has some conceptual overlap with the SDO scales employed in Social Dominance Theory (Sidanius \& Pratto, 1999), including items such as 'We have gone too far in pushing equal rights in this country' and 'Our society would be better off if the distribution of wealth was more equal' (reverse 
coded). ${ }^{8}$ Hartman et al. (2017) included the Hierarchy-Egalitarianism scale in their validation of the Credibility of Science scale and report the two measures are moderately negatively correlated $(r=-.45)$. That is, people who endorse a hierarchical structure of society are less likely to trust science and scientists.

The research above offers some limited empirical evidence that RWA and SDO may be linked to evaluations of science generally. But why would this be the case? Here I offer some theoretical justification for why SDO and RWA should predict negative views of science and scientists.

Socially dominant individuals may be cynical about science and scientists because they perceive scientists as challenging hierarchical social structures. A great deal of scientific research takes place in universities and the public situates science in a university context; $75 \%$ of respondents to the 2006 General Social Survey considered it important that science 'is done by scientists employed in a university setting' (Bainbridge, 2015). This is important, as Social Dominance Theory highlights universities as examples of hierarchy-attenuating social institutions, that is, they function to decrease inequalities (Sidanius, Pratto, Martin, \& Stallworth, 1991; Sinclair, Sidanius, \& Levin, 1998). This can be seen in the case of internal policies, such as equal opportunity scholarships, but also in terms of academics' public policy recommendations and critiques in areas as diverse as environmental regulation and public health. Beyond this, academics, including those working in the sciences, are more likely to express liberal and socially progressive political views, compared to the public (Gross \& Simmons, 2014; Stolzenberg et al., 2019). Sidanius et al. (1991) suggest that, in the context of Social Dominance Theory, higher education is a " subversive' social institution because it serves to decrease beliefs in legitimizing myths and to question the basis of established authority" (p. 715). Douglas (2015) also speculates that hierarchy-valuing individuals are likely to be less trusting of science as it is based on an "epistemic community that values recognizing that each scientist 'stands on the shoulders of giants' (i.e., all the other scientists who came before) and for which community criticism is part of the assurance of reliable knowledge" (p. 298). Thus, we might expect high SDO individuals to be critical of scientists as members of hierarchy-attenuating institutions. This is particularly likely to be the case

\footnotetext{
${ }^{8}$ No published research has compared the scales. However, in an unpublished $2018 \mathrm{NZ}$ student sample $(N=$ 461), Ho et al.'s (2015) $\mathrm{SDO}_{7}$ scale $(M=2.60, S D=0.82, \alpha=.86)$ correlated $(r=.60, p<.001)$ with a 6-item version of the Hierarchy-Egalitarianism scale (Kahan, 2012b) adapted for an Australian population $(M=2.55$, $S D=1.05, \alpha=.81)$
} 
when considering the most divisive rejection of science issues, which largely revolve around public health and environmental contexts and where scientists may be perceived as challenging existing hierarchies (e.g. those supporting vested interests which profit from environmental degradation or social inequality).

There are theoretical reasons to expect that RWA is also associated with negative attitudes towards scientists and science more broadly. Scientists, as a group, may be perceived as challenging established authorities and violating moral norms, two actions anathema to authoritarians. Rutjens and Heine (2016) provide data that support this assumption. Respondents to an online survey perceived scientists (compared to 'regular people'), as less religious and as valuing knowledge, curiosity, and exploration over 'doing the right thing', morality and 'following the norms'. Reinforcing this, scientists were also perceived as less likely to endorse the binding moral foundations outlined in Moral Foundation Theory (Graham et al., 2011): loyalty, authority, and purity. Previous research has mapped moral foundations onto RWA, finding that authoritarians place greater value on the binding foundations (Federico, Weber, Ergun, \& Hunt, 2013; Milojev et al., 2014). Thus, high RWAs may see scientists as radical deviants following a different moral compass.

One can also frame the conflict between ideological attitudes and science in terms of the norms of science. For instance, sociologist Robert Merton identified four key sets of values attached to the idealised pursuit of science: communism, universalism, disinterestedness, and organized scepticism (Merton, 1973; see also Rutjens et al., 2018). We can contrast these norms with values embraced by high RWA and SDO people. Communism, the free sharing of ideas and information, and universalism, the openness of science to all comers, conflict with the competitive and hierarchical beliefs of high SDOs.

Disinterestedness, a lack of ideological loyalty, and scepticism, a questioning of tradition and established authority, conflict with the in-group loyalty and unquestioning obedience to authority of high RWAs. Merton (1973) writes that conflict with the norms of science likely manifests as a general opposition to science which "may exist quite apart from the introduction of specific scientific discoveries which appear to invalidate particular dogmas of church, economy, or state. It is rather a diffuse, frequently vague, apprehension that skepticism threatens the current distribution of power" (p. 278).

To summarise, in the case of those who score higher on RWA and SDO, a perceived lack of shared values with scientists and a perception that scientists threaten hierarchies and challenge authorities could underlie a distrust of science, which in turn leads to rejection of 
science (Fiske \& Dupree, 2014; Hartman et al., 2017; Siegrist, Cvetkovich, \& Roth, 2000; Siegrist, Cvetkovich, \& Gutscher, 2001). Studies 5 and 6 investigate the extent to which RWA and SDO predict trust in scientists, in US and NZ samples. These studies also investigate the degree to which trust in scientists mediates the relationship between RWA, SDO, and rejection of specific scientific findings.

\subsection{CHAPTER SUMMARY AND RESEARCH QUESTIONS}

This chapter reviewed several ways researchers have approached the question of 'why do people reject science?' Researchers have investigated the role of general science knowledge, attitudes towards science, and awareness of the scientific consensus. On this last point, the recently developed GBM (van der Linden, Leiserowitz, et al., 2015) offers an interesting mechanism by which communicators could reduce rejection of science. However, evidence for the model is mixed, and questions remain regarding the applicability of the model to different scientific issues and national contexts. All research on consensus messaging to date has focused on messages describing a high level of consensus - do descriptions of low scientific agreement lead to increased rejection of science? There is also the question of how perceptions of consensus and personal beliefs influence each other over time 'in the wild', i.e. in the absence of experimental manipulations. The first two studies in this thesis seek to answer these questions. Study 1 presents an experiment which examines the effects of messages about either high or low consensus on personal beliefs about climate change or GM foods in a New Zealand student sample. Study 2 takes a different approach to investigate the causal direction of the association between perceptions of consensus and personal beliefs, using a longitudinal design to infer causality.

Moving beyond perceptions of consensus, the cognitive and social psychological literature offers further insights into how deeper beliefs and values can drive rejection of science. Through a process of motivated reasoning (Kunda, 1990), people may draw conclusions that are inaccurate but more desirable in terms of fitting with their prior beliefs and worldviews. The Attitude Roots Model (Hornsey \& Fielding, 2017) offers a simple framework for investigating how deeper values and beliefs drive the 'surface attitudes' of rejection of science.

While many studies have investigated the ideological drivers of rejection of science, the overwhelming majority rely on self-report measures. Study 3 takes a detour from survey 
research to examine the link between political ideology and rejection of science in a behavioural context: who people choose to follow on the social media platform Twitter. I investigate if users who follow right-leaning (vs. left-leaning) New Zealand political accounts are more likely to follow accounts sharing rejection of science content. This study presents a novel approach to studying rejection of science. Assuming that following choices are indicative of personal beliefs, we would expect a positive association between the following of conservative accounts and the following of rejection of science accounts.

In reviewing the research on political ideology and other ideological drivers of rejection of science I make a case that the DPM (Duckitt \& Sibley, 2009) holds promise in predicting rejection across scientific domains. The ideological attitudes RWA and SDO both predict climate change denial (Stanley \& Wilson, 2019), but do they also predict rejection of science regarding other contested scientific issues such as GM food and vaccines? Study 4 aims to answer this question, drawing on a survey of students to investigate the association between ideological attitudes and rejection of science across all three issues. What might explain the relationship between ideological attitudes and rejection of science? The last two studies use survey data from US (Study 5) and New Zealand (Study 6) samples and SEM to explore the myriad paths by which ideological attitudes might influence beliefs about scientific issues. Expanding on the results of Study 4, I include two further issues as rejection of science examples: evolution and fluoridation. To understand why RWA and SDO may be linked with rejection of science, I examine potential mediators of the effects of RWA and SDO, including political ideology, conspiracy mentality, religiosity, and free market beliefs (Lewandowsky, Gignac, et al., 2013; Rutjens et al., 2017). I also incorporate two more science-specific mediating variables which may explain a link between ideological attitudes and rejection of science: trust in scientists and an objective measure of scientific knowledge (Drummond \& Fischhoff, 2017; Hartman et al., 2017). The final model offers a nuanced insight into the attitude roots of rejection of science across multiple issues. 


\section{Chapter 4: Investigating the Gateway Belief Model}

The GBM describes a process of attitudinal change in which shifting peoples' perceptions of a scientific consensus on an issue leads to belief revision in line with scientific consensus, which in turn to leads to changes in support for public action (reviewed in section 3.1.3). This chapter presents two studies examining the GBM in New Zealand student samples.

\subsection{PREFACE}

The focus of my thesis research has been to understand how people form and change their opinions on controversial scientific issues. In the initial stages of my $\mathrm{PhD}$ research, I searched for theories and models which aimed to explain attitudes and beliefs about scientific issues across different domains (i.e. not just focussing on one specific issue). The initial paper outlining the GBM (van der Linden, Leiserowitz, et al., 2015) presented an interesting idea and had received wider attention outside of peer-reviewed literature-often accompanied by strong claims. For example, in a Scientific American article van der Linden and Lewandowsky (2015) wrote "highlighting how many experts agree on a controversial issue has a far-reaching psychological influence.” My interest was piqued. However, the model was not without its detractors. Kahan (2017) criticised the initial GBM study, pointing out that the consensus information intervention did not actually increase self-reported beliefs in climate change relative to a control message. ${ }^{9}$ Effects in the initial study were based on a path model and a difference-in-differences approach (see van der Linden, Leiserowitz, \& Maibach, 2017, for a response). Given the debate over the validity of the model, it seemed a good starting point for focussing my efforts and an opportunity to contribute meaningfully to the nascent empirical literature on the relationship between perceptions of scientific consensus and beliefs about contested science.

The original study describing and testing the GBM focussed on how messages about a high level of scientific consensus (97\%) shift beliefs regarding anthropogenic climate change

\footnotetext{
${ }^{9}$ This critique was first published as a working paper on the Social Science Research Network server in May 2016.
} 
(van der Linden, Leiserowitz, et al., 2015). The first study in this chapter, Study 1, extends the GBM to beliefs about GM food and examines the impact of both high and low consensus messages. At the time this study was conceived, the GBM had been experimentally tested in the context of beliefs about climate change and vaccination, but not GM food (van der Linden, Clarke, et al., 2015; van der Linden, Leiserowitz, et al., 2015). However, Timpona (2015) speculated that perceptions of scientific consensus may also influence beliefs about GM food. Thus, I decided to include GM food beliefs in this study to test this claim. As I was developing the Study 1 survey experiment, Dixon (2016) published a study testing the GBM in the context of GM food safety. Considering this, I decided to use the same GM food safety items as Dixon as a replication of his study.

The following section presents the final version of the full manuscript submitted to, and published in the journal PLOS ONE (Kerr \& Wilson, 2018a), under a Creative Commons Attribution (CC BY) license.

\subsection{STUDY 1: CHANGES IN PERCEIVED SCIENTIFIC CONSENSUS SHIFT BELIEFS ABOUT CLIMATE CHANGE AND GM FOOD SAFETY}

\subsubsection{Abstract}

Despite an overwhelming scientific consensus, a sizable minority of people doubt that human activity is causing climate change. Communicating the existence of a scientific consensus has been suggested as a way to correct individuals' misperceptions about humancaused climate change and other scientific issues, though empirical support is mixed. We report an experiment in which psychology students were presented with consensus information about two issues, and subsequently reported their perception of the level of consensus and extent of their endorsement of those issues. We find that messages about scientific consensus on the reality of anthropogenic climate change and the safety of genetically modified food shift perceptions of scientific consensus. Using mediation models, we also show that, for both these issues, high consensus messages also increase reported personal agreement with the scientific consensus, mediated by changes in perceptions of a scientific consensus. This confirms the role of perceived consensus in informing personal beliefs about climate change, though results indicate the impact of single, one-off messages may be limited. 


\subsubsection{Introduction}

There are some issues for which a substantial proportion of the public hold beliefs that are at odds with broad scientific consensus. Examples include the reality of human-caused climate change (Milfont et al., 2017; Tranter \& Booth, 2015), the safety of genetically modified (GM) food (McFadden, 2016) and the efficacy of vaccines (H. J. Larson et al., 2016). This 'rejection of science' poses a risk to humanity: doubts about climate change lead to personal and societal inaction, resulting in global environmental changes as well as social and economic costs (IPCC, 2014); concerns regarding vaccination threaten to lower immunisation rates and increase the spread of diseases such as measles (H. J. Larson et al., 2016), and; fears around the safety of GM foods could limit efforts to provide a sustainable level of food production for a growing global population (Freedman, 2013).

To varying degrees, public disagreement over these issues exists in New Zealand. A 2014 representative national survey reported that less than half (49\%) of New Zealanders feel certain that climate change is really happening (24\% are undecided and $28 \%$ disagree) (Leining \& White, 2015). Thirty percent of participants in the 2014 wave of the longitudinal New Zealand Attitudes and Values study disagreed or were unsure that humans are causing climate change (Milfont et al., 2017). This disagreement and uncertainty is at odds with the scientific consensus that climate change is occurring and caused by humans. A recent review of studies examining the level of consensus estimates that $97 \%$ of climate scientists agree that climate change is happening and human-caused (Cook et al., 2016). On the topic of GM, the most recent public opinion research suggests that New Zealanders' tend to hold negative attitudes about GM foods and a plurality believe that they are dangerous to human health. In a 2013 random postal survey of New Zealanders, 27\% of respondents were 'totally opposed' to GM and $42 \%$ agreed with the statement: 'GM poses a significant risk to the health and safety of humans' (Chikazhe, 2015). This is despite a number of comprehensive reports finding that GM foods are no different to conventional foods in terms of negative health effects (National Academies of Sciences, Engineering, and Medicine, 2016b; Nicolia et al., 2014). Public opinion data on vaccination is sparse in New Zealand, but a 2012 nationally representative survey of adults reported that $90 \%$ of respondents thought that vaccines were safe. Of the remaining $10 \%$, half the respondents had concerns about vaccine safety and half were unsure (Meningitis Foundation Aotearoa New Zealand, 2013).

Along with personal beliefs regarding these issues, people also hold widely varying views on the level of consensus among scientists. For example, Leining and White's (2015) 
survey found that only 53\% of New Zealand respondents agreed with the statement 'Most scientists agree that humans are causing climate change.' Misperceptions of scientific consensus have been attributed a range of overlapping factors, including: media giving equal balance to both sides of a debate (Koehler, 2016), deliberate misinformation campaigns by interested parties (Maibach, Myers, \& Leiserowitz, 2014) and cognitive biases arising from political and cultural ideologies (Kahan, Jenkins-Smith, \& Braman, 2011). Messages specifically communicating the scientific consensus ('consensus messages') have been suggested as a way to correct such misperceptions as well as shift individuals' personal attitudes about scientific issues (Maibach et al., 2014; Maibach \& van der Linden, 2016).

Consensus messages tap into two heuristics-mental rules-of-thumb-studied in persuasion research: the expert heuristic ('experts can be trusted') and the consensus heuristic ('consensus implies correctness'). Within models of persuasion such as the Heuristic Systematic Model (Chaiken et al., 1989) and the Elaboration Likelihood Model (Petty \& Cacioppo, 1986), heuristics are employed, consciously or subconsciously, to reduce cognitive effort when evaluating a message. Information attributed to an expert (vs. non-expert) source has a greater influence on an individuals' attitudes and opinions. Likewise, information that a position is supported by the majority of a group generally has more influence on attitudes than information stating a position is supported by a minority (Kruglanski \& Stroebe, 2005). A number of studies examining the influence of consensus messages on beliefs draw on the heuristic role of expert consensus information as an explanation for its potential effects on individuals' beliefs about scientific issues (e.g. Bolsen \& Druckman, 2015; Ding, Maibach, Zhao, Roser-Renouf, \& Leiserowitz, 2011; Dixon, McKeever, Holton, Clarke, \& Eosco, 2015; van der Linden, Leiserowitz, et al., 2015).

There are a growing number of experimental studies investigating the efficacy of consensus messages in changing perceptions and beliefs. These have primarily focussed on climate change but also touch on other scientific domains.

\section{Changing perceptions of scientific consensus and personal beliefs}

\section{Climate change}

Cross-sectional studies typically show a positive relationship between the perception that there is widespread agreement among climate scientists and personal belief that climate change is occurring (Ding et al., 2011; Hornsey et al., 2016; McCright, Dunlap, \& Xiao, 2013). A number of experimental studies have found that exposing individuals to a simple message incorporating the ' $97 \%$ ' figure in various forms increases participant perceptions of 
scientific consensus, most commonly operationalised as an estimate of the percentage of scientists agreeing that climate change is occurring (Deryugina \& Shurchkov, 2016; Lewandowsky, Gignac, \& Vaughan, 2012; Myers, Maibach, Peters, \& Leiserowitz, 2015; van der Linden, Leiserowitz, Feinberg, \& Maibach, 2014). Studies have also shown that perception of consensus among climate scientists can be decreased by reading information from sceptic organisations claiming there is a lack of consensus (van der Linden, Leiserowitz, Rosenthal, et al., 2017), reading news reports giving disproportionate balance to sceptical viewpoints (Cook et al., 2017) or by watching a sceptical 'climate conspiracy' video (van der Linden, 2015b). Taken together, this research shows that individuals' perceptions of a scientific consensus regarding climate change can be manipulated by relatively simple interventions.

Taking the next logical step, social psychologists have examined the possibility that changing peoples' perception of the scientific consensus leads to changes in their personal belief that human-caused climate change is occurring. In an experiment, van der Linden and colleagues (van der Linden, Leiserowitz, et al., 2015) show that reading messages about the $97 \%$ consensus significantly increases individuals' estimates of scientific agreement on the reality of human-caused climate change. They use a path modelling approach to argue that these changes are, in turn, associated with increases in belief in human-caused climate change, concern over climate change and support for public action. The authors present their results as evidence supporting a 'Gateway Belief Model' (GBM), with perceptions of consensus acting as a key cognition influencing other beliefs. They write:

...we find that increasing public perceptions of the scientific consensus is significantly and causally associated with an increase in the belief that climate change is happening, human-caused and a worrisome threat. In turn, changes in these key beliefs are predictive of increased support for public action. In short, we find that perceived scientific agreement is an important gateway belief, ultimately influencing public responses to climate change. (p.1)

Similar experiments have demonstrated that informing individuals about the high level of scientific consensus significantly increases reported personal belief that humancaused climate change is occurring (Cook \& Lewandowsky, 2016; Lewandowsky et al., 2012; van der Linden, Leiserowitz, \& Maibach, 2017). Conversely, several studies have reported that exposure to a consensus message may increase perceptions of scientific consensus, but has no effect on belief in the reality of climate change (Bolsen \& Druckman, 
2018a; Bolsen, Leeper, \& Shapiro, 2014; Dixon et al., 2017; Kobayashi, 2018; van der Linden et al., 2014).

\section{Genetically modified food}

Like climate change, GM food safety is a topic of intense public debate (Harker, 2015; Lang, 2016). However, only one study to date has examined the role of consensus information in changing beliefs. Applying the GBM to the topic of genetic modification, Dixon (2016) reports in two studies that exposure to a simple message stating " $90 \%$ of scientists believe that GM foods are safe to consume" increases participants' perceptions of a scientific consensus on the issue, but, at a broad level, has no impact on their personal beliefs about GM food safety. Examining the data further, Dixon reports moderated mediation models describing the effect of a consensus message on beliefs mediated via perceptions of consensus. Here he reports a significant indirect effect of consensus message on beliefs, mediated via perceptions of consensus. This indirect effect was moderated by prior beliefs, such that the consensus message had stronger effect on beliefs among participants with more positive prior attitudes towards GM food, and a weaker (in one study non-significant) effect for those with negative prior attitudes towards GM foods.

\section{Other issues}

While the majority of attention to consensus messaging has focused on climate change, studies have also explored other scientific issues. In support of the GBM, studies have found that interventions which change participants' perception of a scientific consensus also change their personal beliefs in relation to vaccination (Dixon \& Clarke, 2013; Dixon et al., 2015; van der Linden, Clarke, et al., 2015), pharmaceutical pollution of waterways (Dunwoody \& Kohl, 2017), scientific whale research and the link between blood type and personality (Kobayashi, 2018).

\section{Current study}

At the heart of the GBM is the causal link between perceptions of scientific consensus and personal beliefs. While consensus information can shift perceptions of consensus, absence of a subsequent effect on personal beliefs in some studies (Bolsen et al., 2014; Dixon, 2016; Dixon et al., 2017) calls into question this causal link, and the overall efficacy of consensus messaging as an approach to changing public opinion on climate change (Kahan, 2017; Pearce et al., 2017b). Further studies examining the role of perceived consensus across different scientific issues can add to this body of research, offering 
empirical insights building towards a clearer understanding of the role of consensus information in informing beliefs about scientific issues. Indeed, a recent report from the National Academy of Sciences has called for more research into how, and when, consensus information can shift individuals' opinions, as well as comparison of communication approaches across differing national contexts (National Academies of Sciences, Engineering, and Medicine, 2016a).

Here we present a basic test of the GBM in a New Zealand student (convenience) sample, experimentally investigating the impact of simple consensus messages on selfreported personal beliefs, and the mediating role of perceptions of scientific consensus. The study examines two separate scientific issues where a scientific consensus exists but there is public uncertainty: climate change and GM food safety. Climate change was selected as it is the issue on which the GBM was initially based, and there are a number of conflicting findings in the literature (e.g. Dixon et al., 2017; Kobayashi, 2018). GM food safety was selected as there is only one study to date examining the GBM in this context (Dixon, 2016). While a number of studies have used consensus messages relating a specific high percentage value to describe the level of consensus e.g. ' $97 \%$ of climate scientists agree...', no studies investigating climate change or GM food beliefs have examined the effect of a similar message outlining a relatively low percentage. Does low consensus information have an identical but opposite effect on beliefs? In the current study we include both high (97\%) and low $(63 \%)$ consensus messages to answer this question.

Based on the research reviewed above, we expect that messages detailing a high or low level of agreement on GM food safety and climate change will respectively increase or decrease (compared to controls) participants' perceptions of scientific consensus on these issues. Drawing on the GBM, we also expect that these messages will have an effect on participants' beliefs about climate change and GM food safety. We predict that high consensus messages will increase participant's agreement with the consensus position - that climate change is happening or that GM food is safe to eat - relative to a control group who read no message. Similarly, we predict that low consensus messages will decrease these beliefs. Lastly, we also predict that any effects of consensus messages on beliefs (specifically belief in human-caused climate change or belief that GM food is safe) can be explained by changes in perceptions of consensus. This hypothesis will be examined using statistical mediation models (Jose, 2013). 


\subsubsection{Method}

\section{Participants}

A total of 696 participants took part in the study: 509 women (73.1\%), 179 men $(25.7 \%)$ and eight individuals reporting non-binary or no gender (1.2\%). Participants were enrolled in an introductory psychology course at Victoria University Wellington and voluntary completion of the study counted as partial course credit towards required research participation/summarisation hours. Ages ranged from 17 to 52 years of age $(M=18.86, S D=$ 2.99). All participants provided informed consent via an electronic form and were fully debriefed at the conclusion of the study. The study was approved by the Victoria University of Wellington School of Psychology ethics committee (application number: 0000023961).

\section{Materials}

Participants competed an online survey which contained an experimental manipulation. Participants read one of several consensus messages, or an attention check in the control condition (3 participants failing this check were removed). In the consensus message conditions, participants were presented with a single simple statement relating a high $(97 \%)$ or low $(63 \%)$ level of consensus regarding the reality of climate change or the safety of GM food. The climate change consensus messages read: "Did you know? A recent survey shows that [97\%][63\%] of climate scientists agree that climate change is occurring and caused by humans." The GM messages were: "Did you know? A recent survey shows that [97\%][63\%] of food scientists agree that genetically modified food is safe to eat." These statements were modelled on previous studies, which found that short simple statements significantly influence participants' estimates of consensus (Dixon, 2016; Myers et al., 2015; van der Linden et al., 2014). The 'high' value of $97 \%$ consensus was selected as it reflects current estimates of scientific consensus regarding climate change (Cook et al., 2016). Previous data from this student population indicate the mean estimate of scientific consensus among climate scientists to be approximately $80 \%$ (M. S. Wilson, 2015) thus the figure of $63 \%$ was selected as the 'low' figure, being equidistant from this mean. As there were no prior data available for estimates of the level of scientific consensus on GM food safety in this population, the $97 \%$ and $63 \%$ values were used for consistency in an exploratory capacity. Participants who read a consensus statement also completed an attention check on the same page as the statement, entering a number in response to the following request: To make sure you understood the text, could you please enter the percentage of scientists in 
agreement according to the statement above. Participants who failed this check were removed from the sample (11 participants).

Both before and after the experimental manipulation, all participants were asked to provide an estimate of the percentage of climate scientists agreeing "that climate change is happening and caused by humans" and the percentage of food scientists agreeing "that food made from genetically modified plants and animals is safe to eat." Responses were captured on a sliding scale ranging from $0 \%$ to $100 \%$, with the marker anchored at $50 \%$. Pre-treatment items provided a randomisation check as well as a baseline to ensure that the experimental manipulation did change perceptions of consensus. Following the experimental manipulation, participants completed a filler section comprising a shortened version of the National Science Foundation Science Literacy Scale (Kahan et al., 2012), to increase the separation between the experimental manipulation and items capturing post-treatment consensus estimates and outcome variables (Podsakoff, MacKenzie, Lee, \& Podsakoff, 2003). Post-treatment consensus estimate items were embedded in a battery of six items asking participants to estimate the percentage of scientists agreeing with a range of statements that reflect debated science. These extra items were included to increase the psychological separation between the experimental manipulation and post-treatment GM/climate consensus items (Podsakoff et al., 2003).

Lewandowsky, Gignac and Oberauer's (2013) 5-item scale was used to measure participants' belief that human-caused climate change is occurring (example item: Human CO2 emissions cause climate change). Responses were captured on a 7-point Likert scale ranging from 1 (Strongly disagree) to 7 (Strongly agree). The scale displayed acceptable internal consistency (Cronbach's $\alpha=.72$ ) according to the criteria suggested by George and Mallery (2003). For comparison, Lewandowsky et al. report an $\alpha$ value of .78 for this scale.

Dixon's (2016) 3-item scale was used to measure belief that GM food is safe to eat (example item: GM foods are safe to eat). Responses were captured on a 7-point Likert scale ranging from 1 (Strongly disagree) to 7 (Strongly agree). The scale displayed good reliability $(\alpha=.85)$ slightly lower than that reported by Dixon $(\alpha=.91)$.

The GBM extends beyond simple alignment of beliefs with scientific consensus; research also suggests that shifting perceptions of consensus influences concern, support for policy and behavioural intentions (van der Linden, Clarke, et al., 2015; van der Linden, Leiserowitz, et al., 2015). A number of other scales covering these constructs were included in the survey (presented after the measures above) but are not reported here for brevity. 
Details of these scales and comparisons between experimental groups can be found in Appendices A and B.

\section{Procedure}

Participants registered to undertake the survey using a SONA Systems (Bethesda, MD) interface and completed the survey online via the SurveyMonkey platform (www.surveymonkey.com, San Mateo, CA) in their own time over a seven-day period. Participants were randomised to one of five conditions: reading a statement about a high or low level of scientific consensus regarding climate change or GM food, or a control condition. All participants completed all measures (a full list of all survey items can be found in Appendix A). Data were analysed using SPSS v23.0 and mediation was tested using Hayes' (2012) PROCESS macro for SPSS.

\subsubsection{Results}

\section{Effects of consensus messaging on perceptions of consensus}

\section{Climate change}

Mean estimates of consensus before and after reading a message about high (97\%) or low (63\%) consensus among climate scientists, or no message, are shown in Table 4.1. The distribution of consensus estimates and scores on the climate change belief scale, by condition, are shown in Figure 4.1.

Table 4.1

Mean estimates of consensus among climate scientists (SD) before and after reading climate change consensus messages.

\begin{tabular}{lllllc}
\hline & $N$ & \multicolumn{2}{c}{ Pre-treatment estimate } & \multicolumn{2}{c}{ Post-treatment estimate } \\
\hline High (97\%) climate message & 142 & 79.35 & $(15.75)$ & $92.34^{\mathrm{a} *}$ & $(11.97)$ \\
Low (63\%) climate message & 151 & 78.89 & $(17.27)$ & $67.49^{\mathrm{a} *}$ & $(11.74)$ \\
Control & 122 & 79.61 & $(15.24)$ & $80.17^{\mathrm{a}}$ & $(16.87)$ \\
\hline
\end{tabular}

*Significant difference between pre- and post-treatment estimates, $p<.001$. Matching superscripts indicate significant differences between conditions at each time point, $p<.001$. 

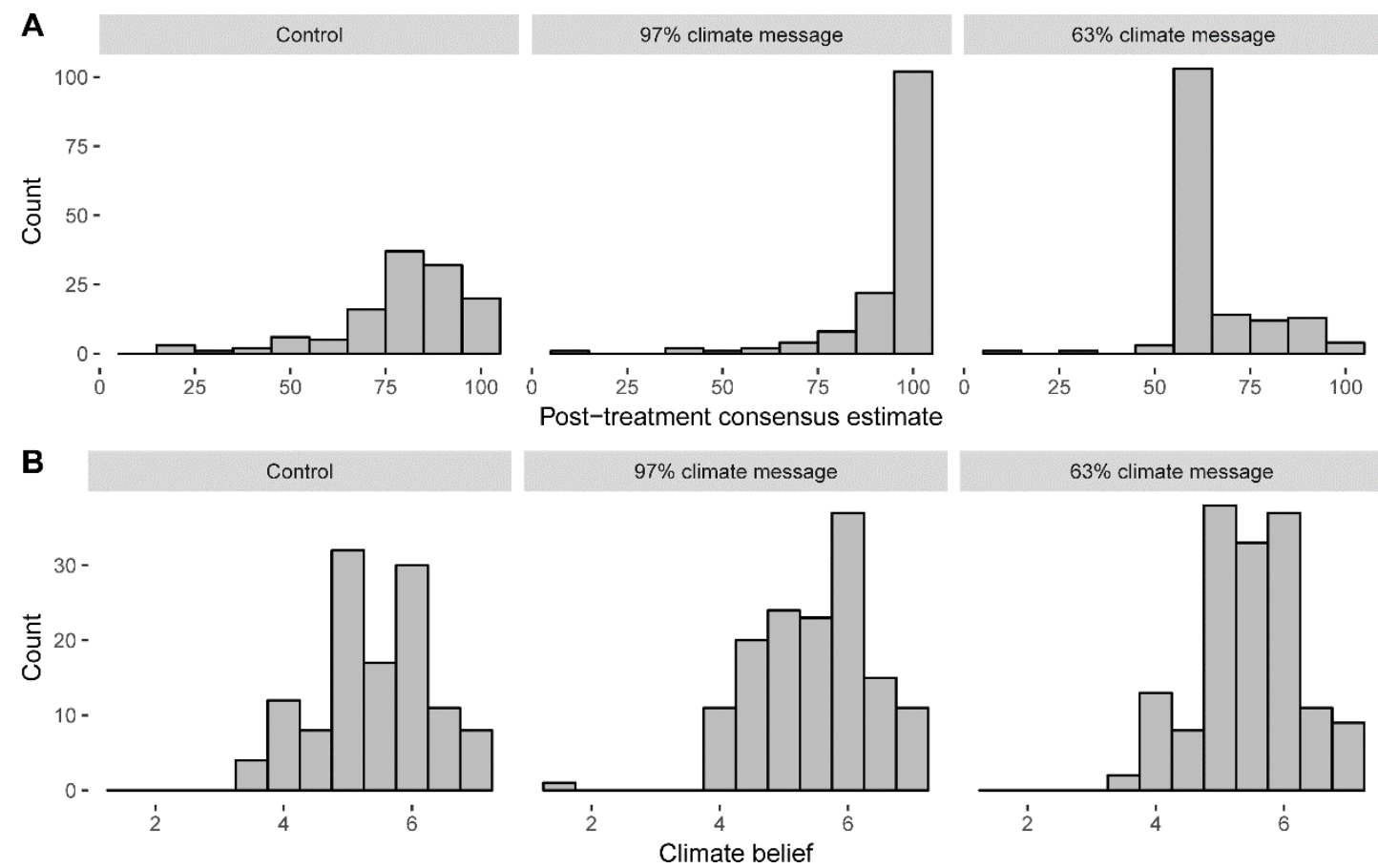

Figure 4.1. Distribution of post-treatment estimates of scientific consensus on climate change (A) and average score on climate change belief scale in experimental conditions (B).

We examined the effects of climate change consensus messages on estimates of agreement among scientists. A 2 (time: pre- vs post-treatment) x 3 (condition: high, low vs control messages) mixed measures ANOVA found a significant main effect of condition $F(2$, $412)=36.80, p<.001, \eta_{\mathrm{p}}{ }^{2}=.15$. Post-hoc tests with Bonferroni correction indicated that the mean estimates of consensus (pre- and post-treatment combined) for all conditions were significantly different from each other ( $p<.001$ in all cases), with estimates in the high consensus condition higher than those in the control condition and those in low consensus condition lower than the control condition. While there was no main effect of time, $F(1,412)$ $=0.84, p>.1$, there was a significant interaction between time and message condition, $F(2,412)=87.04, p<.001 . \eta_{\mathrm{p}}{ }^{2}=0.30$. We followed up the significant interaction with posthoc tests. As expected, there was no significant difference between pre- and post-treatment estimates of consensus in the control condition $t(121)=0.51, p>.1$. In the high consensus message condition, post-treatment estimates of consensus were higher than pre-treatment estimates $t(141)=8.74, p<.001, d=.92$. In the low consensus message condition, posttreatment estimates of consensus were lower than pre-treatment estimates, $t(150)=8.44, p$ $<.001, d=.92$. Univariate analyses indicated no significant difference across conditions in pre-treatment estimates, $F(2,412)=0.07, p>.1$, and a significant difference between groups 
for post-treatment estimates $F(2,412)=123.81, p<.001, \eta_{\mathrm{p}}^{2}=.37$. Comparisons of posttreatment estimates with Bonferroni correction revealed that estimates in the high consensus message condition were higher than those in the low and control conditions and estimates in the low condition were lower than those in the control condition ( $p<.001$ in all cases). In sum, reading messages about high or low consensus among climate scientists, respectively, increases or decreases perceptions of consensus.

\section{GM Food safety}

Mean estimates of consensus before and after reading a message about high (97\%) or low (63\% ) GM consensus among food scientists, or no message, are shown in Table 4.2. The distribution of consensus estimates and scores on the GM food safety beliefs scale, by condition, are shown in Figure 4.2.

Table 4.2

Mean estimates of consensus among food scientists (SD) before and after reading GM consensus messages.

\begin{tabular}{lccccc}
\hline & $N$ & \multicolumn{2}{c}{ Pre-treatment estimate } & \multicolumn{2}{c}{ Post-treatment estimate } \\
\hline High (97\%) GM message & 131 & $56.95^{\mathrm{b}}$ & $(24.03)$ & $87.30^{\mathrm{a} *}$ & $(19.39)$ \\
Low (63\%) GM message & 136 & $63.95^{\mathrm{a}}$ & $(24.33)$ & $64.25^{\mathrm{a}}$ & $(8.70)$ \\
Control & 122 & 57.31 & $(22.07)$ & $57.56^{\mathrm{a}}$ & $(23.16)$ \\
\hline
\end{tabular}

*Significant difference between pre- and post-treatment estimates, $p<.001$. Matching superscripts indicate significant differences between conditions at each time point, $p<0.05$. 

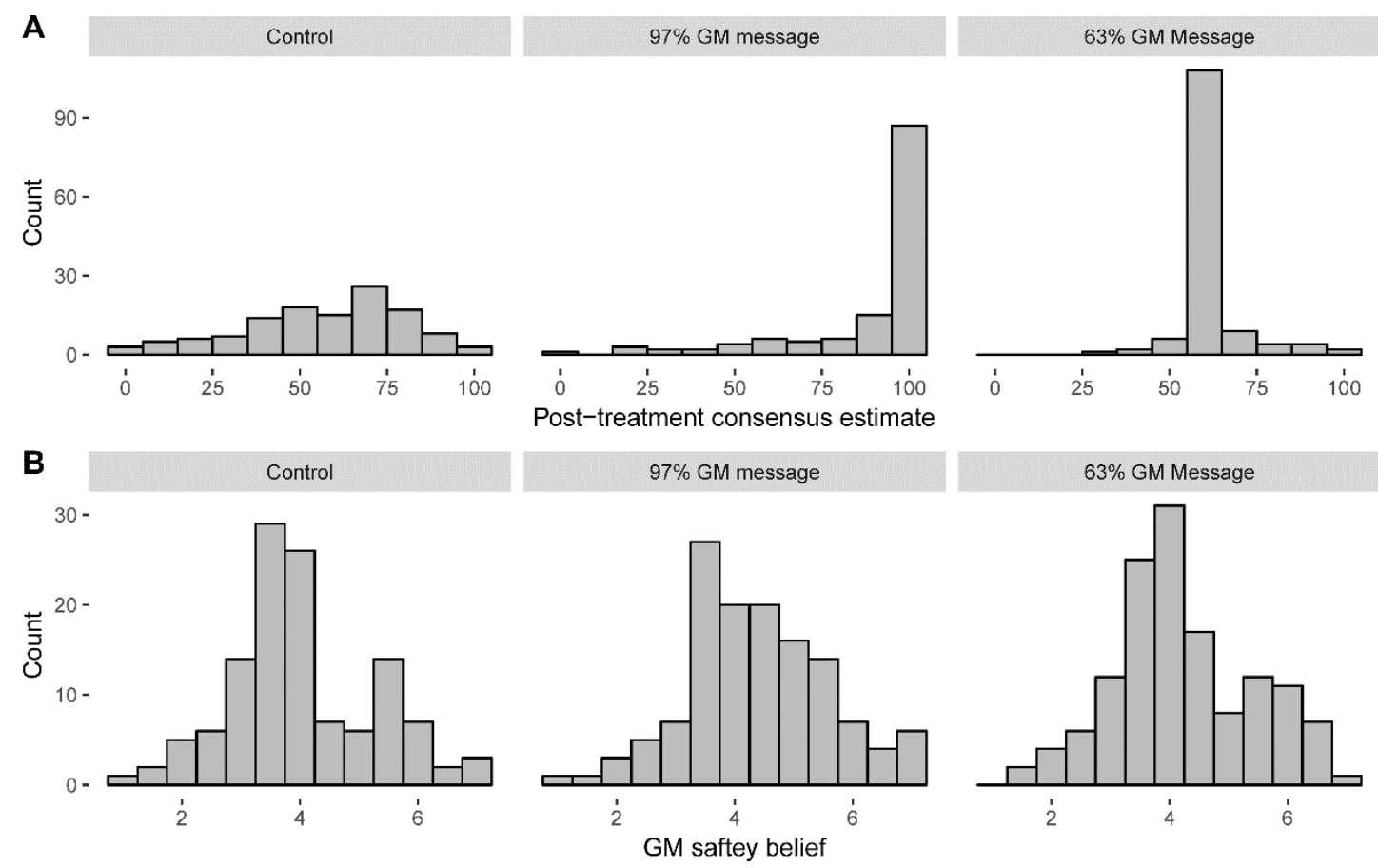

Figure 4.2. Distribution of post-treatment estimates of scientific consensus on GM food safety (A) and average score on GM food safety beliefs scale in experimental conditions (B).

A 2 (time: pre- vs post-treatment) x 3 (condition: high, low vs control messages) mixed measures ANOVA showed a significant main effect of message condition $F(2,386)=$ 22.06, $p<.001, \eta_{\mathrm{p}}{ }^{2}=.10$. Post-hoc tests with Bonferroni correction indicated that the mean estimates of consensus in the high consensus message group were higher than those in the low message $(p<.001)$ and control $(p<.001)$ conditions. Mean consensus estimates in the low consensus message condition were also significantly higher than those in the control condition $(p<.01)$. There was a significant main effect of time $F(1,386)=81.05, p<.001$, $\eta_{\mathrm{p}}{ }^{2}=.17$, indicating a significant difference between pre- and post-treatment scores across all groups combined. There was also a significant interaction between time and condition, $F(2,386)=77.29, p<.001, \eta_{\mathrm{p}}^{2}=.29$. We followed up this interaction with paired-samples $\mathrm{t}-$ tests comparing pre- and post-treatment consensus estimates in each message condition separately. Post-treatment consensus estimates were higher than pre-treatment estimate in the high consensus message condition, $t(130)=12.58, p<.001, d=1.38$. There was no significant difference between pre- and post-treatment consensus estimates in the control condition, $t(121)=0.20, p>.1$, or in the low consensus message condition $t(135)=0.15 p>$ .1. A one-way ANOVA indicated a significant effect of message condition for post-treatment consensus estimates, $F(2,386)=97.40, p<.001, \eta_{\mathrm{p}}{ }^{2}=.33$. Post hoc comparisons indicated 
that both the high $(p<.001)$ and low $(p<.01)$ consensus message groups had higher estimates of scientific consensus compared to the control group and were significantly different from each other $(p<.001)$. A significant difference was observed between conditions for pre-treatment estimates, $F(2,386)=3.72, p=.02, \eta_{\mathrm{p}}{ }^{2}=.02$. Post hoc comparisons showed that pre-treatment estimates of the scientific consensus on GM food safety were significantly higher in the low consensus message condition compared to the high consensus message condition $(p=.047)$. The difference between the low and control conditions was marginally significant $(p=.073)$, with estimates in the low condition higher than those in the control condition. Overall these results indicate that reading a message about high scientific consensus on the safety of GM food among food scientists increases individuals' perceptions of scientific agreement on the issue. Given that the 'low' GM consensus message in fact described a level of consensus close to pre-treatment estimates and did not significantly alter participant's perceptions of consensus relative to pre-treatment estimates, we remove this group from subsequent analyses (combining the no message group and the low GM consensus group into a single control group did not substantially alter results, see Table C.4, Appendix C ).

\section{Effects of consensus messages on beliefs.}

A one-way ANOVA indicated no significant differences in scores on the climate change beliefs scale between participants who read a high consensus climate message $(M=$ $5.47, S D=0.88)$, those who read a low consensus message $(M=5.44, S D=0.80)$ and the control group $(M=5.44, S D=0.80 ; F(2,412)=0.26, p>.1)$. For GM food safety beliefs, an independent samples t-test revealed that participants who read a high consensus message reported higher scores on the GM food safety beliefs scale $(M=4.37, S D=1.12)$ than those who read a control message $(M=4.02, S D=1.23 ; t(251)=-2.31, p=.02)$. This indicates that reading a message about high or low consensus among climate scientists had no impact on reported belief in human-caused climate change but reading a message about the high level of consensus on the safety of GM food among food scientists increased self-reported personal belief that GM food is safe to eat.

\section{Mediation analyses}

We undertook mediation analyses to examine if consensus messages had an indirect effect on beliefs, mediated via perceptions of consensus. Mediation allows the investigation of the effect of an independent variable $(X)$ on a dependent variable $(Y)$ through a third 
'mediating' variable $(M)$, based on a series of regression analyses. The indirect effect $a b$ is the product of the effect of $X$ on $M(a)$ and the effect of $M$ on $Y$ accounting for $X(b)$. Any effect of $X$ on $Y$, accounting for $M$, is termed the direct effect ( $\left(c^{\prime}\right)$ and the overall effect of $X$ on $Y$ is termed the total effect $(c)$. Earlier guidelines for mediation from Baron and Kenny's (1986) 'causal steps' approach recommend only undertaking analyses to examine the role of a mediating variable when there is a significant total effect (i.e. a significant relationship between the independent and dependent variable; path $c$ ). Here we follow more recent guidelines which reject this approach and propose that mediation is a useful tool in identifying mediating relationships even in the absence of a total effect (Hayes, 2009; Rucker, Preacher, Tormala, \& Petty, 2011). Each model presented below separately considers the effects of a message condition compared to the control group as the independent variable, coded dichotomously (message condition $=1$, control $=0$ ). Here we also control for pretreatment estimates of consensus, thus we are measuring how post-treatment changes in perceived consensus relate to climate change belief. To our knowledge, this approach has not been employed in previous mediation models examining the role of perceived consensus in influencing beliefs (e.g. Brewer \& McKnight, 2017; Myers et al., 2015). We note that van der Linden et al. (van der Linden, Leiserowitz, et al., 2015) used pre-post score differences for all variables in their path model. The current study captured pre- and post-test consensus estimates (as a within-subjects manipulation check) but only post-test responses to the climate change and GM belief items.

\section{Climate change}

A mediation model examining the effect of a $97 \%$ climate message on climate beliefs, via perceptions of consensus, found a significant effect of the message on the mediator, which in turn was significantly associated with belief in human-caused climate change (Figure 4.3; full details of each mediation model are reported in Appendix C).

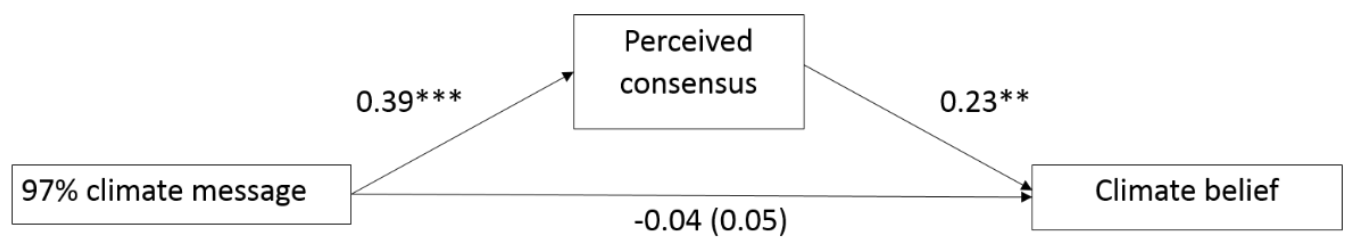

Figure 4.3. The effect of a $97 \%$ climate message on belief in human-caused climate change, mediated via perceptions of consensus among climate scientists, controlling for pre-treatment estimates. Standardized regression coefficients shown $(N=264) .{ }^{* *} p<.01,{ }^{* * *} p<.001$. 
The unstandardized indirect effect, $a b=.16,95 \%$ CI $[.05, .27]$, was significant: the lower and upper values of a $95 \%$ bias-corrected bootstrap confidence interval (5000 samples) did not include zero (Hayes, 2009). Interpreted in practical terms, this means the shift in perceived consensus caused by reading a message about high scientific consensus is associated with a 0.16 increase in climate change endorsement captured on a 7-point scale. We also report here the partially standardised indirect effect, $a b_{p s}$ which describes the indirect effect of a one unit change in $X$ expressed in terms of the standard deviation of the outcome variable $Y$. As the independent variable in the mediation model is dichotomous, this approach provides greater ease of interpretation than the completely standardised indirect effect, $a b_{c s}$ (Preacher \& Kelley, 2011). The partially standardised indirect effect, $a b_{p s}=.19,95 \%$ CI [.05, .32], implies that reported belief in human-caused climate change increases by approximately one-fifth of a standard deviation due to the indirect effect of reading a high consensus message, via changes in perceptions of consensus.

The mediation model for the low climate consensus condition is detailed in Figure 4.4. Reading a message about a low (63\%) level of agreement among climate scientists did not have a significant indirect effect on belief that human-caused climate change is occurring, $a b=-.09,95 \%$ CI $[-.20, .02] ; a b_{p s}=-.11,95 \%$ CI $[-.26, .03]$. This indicates that changes in estimated consensus caused by reading a low consensus message do not have a subsequent effect on belief that human-caused climate change is occurring.

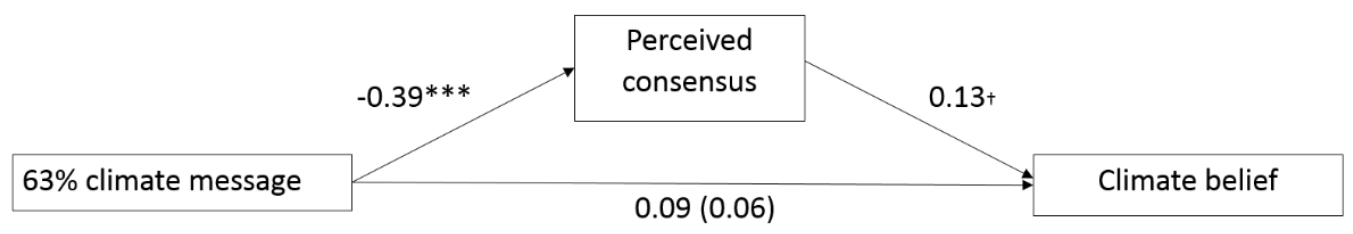

Figure 4.4. The effect of a $63 \%$ climate message on belief in human-caused climate change, mediated via perceptions of consensus among climate scientists, controlling for pre-treatment estimates. Standardized regression coefficients $(\mathrm{N}=273) . \dagger p<.10, * * * p<001$.

\section{GM Food safety}

Recall that, as the exploratory 'low' GM consensus message conveyed a figure close to the participants' existing estimates of scientific, we chose not to further analyse the low consensus GM condition data. The mediation model for the high GM consensus condition is outlined in Figure 4.5. Reading a message about a high (97\%) level of agreement among food 
scientists on the safety of GM food had a significant positive indirect effect on beliefs about the safety of GM food, $a b=0.39,95 \%$ CI $[0.18,0.62] ; a b_{p s}=0.35,95 \%$ CI $[.17, .55]$. Reading a high consensus message increased participants' estimates of the scientific consensus, which in turn was associated with greater belief that GM food is safe. The partially standardised indirect effect indicates that the reading a high consensus GM message led to approximately a third of a standard deviation shift in GM food safety beliefs, mediated via perceptions of consensus.

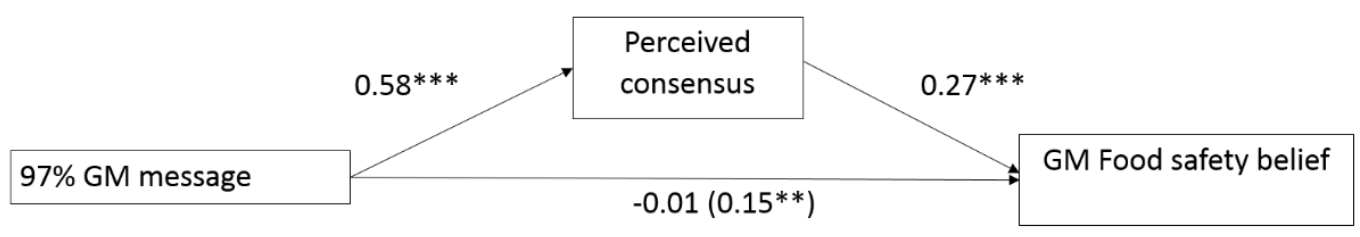

Figure 4.5. The effect of a 97\% GM message on belief that GM food is safe to eat, mediated via perceptions of consensus among GM scientists, controlling for pre-treatment consensus estimates. Standardized regression coefficients shown $(\mathrm{N}=253) . * * p<.01, * * * p<.001$.

In summary, for both the climate change and GM food issues, mediation models revealed an indirect effect of high consensus information on scientific beliefs in the expected direction. Reading messages about high consensus among scientists increases estimates of scientific consensus. Post-treatment estimates of consensus, controlling for the experimental manipulation and pre-treatment estimates of consensus, are significantly positively associated with personal beliefs aligning with the scientific consensus, supporting the GBM. Reading a message outlining a low level of scientific consensus (63\%) did not have a significant total or indirect effect on belief that human-caused climate change is occurring.

\subsubsection{Discussion}

This study offers new insights into the efficacy of consensus messaging and the role that perceptions of scientific agreement play in informing personal beliefs. The GBM posits that people's beliefs about scientific issues are influenced by their perceptions of what scientists believe (van der Linden, Leiserowitz, et al., 2015). It follows that interventions which change an individual's perception of a scientific consensus on a given issue would also change their beliefs about the issue. We used an experimental approach to investigate the effect of consensus messages on participants' perceptions of a scientific consensus on, and beliefs relating to, climate change and the safety of GM food. 
Our results indicate that short, simple messages about high scientific consensus increase individuals' perception of a scientific consensus. The mean estimate of scientific agreement on climate change increased from approximately $79 \%$ to $92 \%$ for participants who read a high consensus message. This replicates results from previous studies (Deryugina \& Shurchkov, 2016; Dixon, 2016; Lewandowsky et al., 2012; Myers et al., 2015; van der Linden et al., 2014; van der Linden, Leiserowitz, \& Maibach, 2018b). In the case of climate change, we also observed a surprisingly symmetrical effect for messages about relatively low consensus (mean estimates decreasing from 79\% to 67\%). Quantitative information about the percentage of scientists endorsing the reality of climate change appears to increase or decrease perceptions of agreement in a roughly equal manner. Regardless of any subsequent effects on beliefs, the current results support the conclusion that messages about the scientific consensus are effective in correcting misperceptions about the actual level of scientific agreement on climate change. This is a worthwhile endpoint in itself, as highlighted in recent studies investigating the effects of climate sceptic communications seeking to decrease public perception of scientific consensus (Cook et al., 2017; van der Linden, Leiserowitz, Rosenthal, et al., 2017).

Despite the results showing that messages about scientific agreement on climate change significantly influence perceptions of consensus, we did not find that consensus messages had a total effect on climate change beliefs, i.e. we did not find a significant relationship between reading or not reading the high consensus climate message and belief in climate change. On its own, this result suggests that simple messages about the level of agreement among climate scientists have no influence on individuals' belief in the reality of human-caused climate change. We should note that our between-subjects design (measuring climate change beliefs only after the experimental manipulation) has lower power to detect a direct effect of message condition on beliefs, compared to a within-subjects, pre-post design (see van der Linden, Leiserowitz, et al., 2015; van der Linden, Leiserowitz, \& Maibach, 2017). A further consideration is the possibility of a functional ceiling effect. Our control group appeared to have a relatively high acceptance of climate change (discussed further below). While there was certainly capacity for higher scores to be recorded on the scale for most participants in the high consensus message condition (see Figure 4.1), it is possible that few participants are willing to report greater agreement with all items in the climate change acceptance scale - even if they strongly believe that human-caused climate change is occurring. Although we do not report a main effect of message condition on climate beliefs, our mediation analyses found that a high climate consensus message did have a significant 
indirect effect; reading a message significantly influenced perceptions of consensus, which in turn influenced key beliefs. This mediated effect of high consensus messages has been noted in several previous experiments, most notably in the path analysis presented in the seminal GBM study (van der Linden, Leiserowitz, et al., 2015) but also others (Brewer \& McKnight, 2017; Myers et al., 2015). Our mediation model examining low climate consensus message effects did not support the GBM, however. We find that reading a low consensus climate message decreased perceptions of consensus, but that this change did not have a negative effect on reported belief that human-caused climate change is occurring.

Previous studies which found no effect of consensus messages on climate change beliefs have only examined the total effect of message condition on beliefs and did not include perceived consensus as a mediator of this effect (Bolsen et al., 2014; Dixon et al., 2017). We note that mediation analyses may be better powered to detect an indirect effect compared to a total effect (O'Rourke \& MacKinnon, 2015; Rucker et al., 2011). If we take a literal view of the GBM, then the absence of a total effect in our study might be taken as indicating that a simple, one-off message about consensus may not have in itself much practical value in changing beliefs. However, the results of the current study confirm the theoretical causal path outlined in the Gateway Belief Model: perceptions of consensus play a role in informing beliefs about climate change - at least for high consensus messages. More engaging or detailed interventions highlighting the scientific consensus on climate change (e.g. Brewer \& McKnight, 2017) may hold greater promise as potential communication strategies. Likewise, repeated exposure to the '97\%' message could be expected have a greater impact on beliefs (Schwarz, Newman, \& Leach, 2016; Weaver, Garcia, Schwarz, \& Miller, 2007). Messages about the scientific consensus also compete with counterclaims that there is not a scientific consensus - often driven by vested interests (Dunlap \& McCright, 2011; Oreskes \& Conway, 2010). Combining consensus messages with 'inoculating' warnings about incorrect counterclaims can prevent misinformation from neutralizing the effects of consensus messages (Cook et al., 2017; van der Linden, Leiserowitz, Rosenthal, et al., 2017). At a national level, there is some evidence supporting the efficacy of consensus messaging. Hamilton (2016) documents a growing awareness of the scientific consensus and acceptance of human-caused climate change in the US in recent years, "compatible with the proposition that implicitly or explicitly communicating evidence of agreement among scientists encourages public acceptance of [anthropogenic climate change] itself” (p. 9)

The mediating role of perceived consensus is apparent when considering messages about GM food safety. Here, the significant total effect of a high consensus message on GM 
food safety belief is explained by effects mediated via perceptions of consensus. This finding supports the generalisation of the GBM to scientific domains other than climate science, in agreement with van der Linden et al. (2015), who found that consensus messages influenced beliefs about vaccine safety via perceptions of consensus. Future research should further examine the role of perceived scientific agreement in other science-related societal debates where public opinion diverges from scientific consensus, for example, community water fluoridation (Allukian et al., 2018). Our GM message results, to some extent, align with the only previous study examining consensus messaging in the context of GM food safety. Dixon (2016) reported that a high consensus message had no overall effect on GM food safety belief (measured using the same scale as the current study). However, Dixon did report a significant indirect effect of a simple message on beliefs via perceptions of consensus (study 2). While we also report an indirect effect, we found a significant main effect of message condition on GM beliefs where Dixon does not. A potential explanation for the divergent findings is the nature of the consensus message; Dixon's consensus message outlined that " $90 \%$ of scientists believe GM foods are safe to consume," while the current study used a higher figure of $97 \%$. Future studies could compare the effects of several differing levels of high consensus (e.g. 90\% vs 97\%) on beliefs about GM foods (see Aklin \& Urpelainen, 2014; Johnson, 2017; Myers et al., 2015). In the context of genetically modified food, simple messages highlighting consensus may be an effective way of increasing public agreement with current consensus on GM food safety. Although we must acknowledge that the high consensus figure used in the GM message was selected to correspond with the climate change figure and is unlikely to reflect the current (and as-yet unquantified) level of scientific agreement on the safety of GM food. It is important to also note that the current study focused solely on the issue of GM food safety, for which there exists a reasonably strong scientific consensus (National Academies of Sciences, Engineering, and Medicine, 2016b). The overarching issue of acceptance of genetically modified organisms rests on wider considerations, including environmental and economic impacts (Sjöberg, 2004).

Several limitations of the study must be acknowledged. Firstly, this study was undertaken in a student sample and the results may not be generalisable to the wider population. For example, women were over-represented in our sample. Student samples are also typically more liberal than the general population, and political ideology is a predictor of opinions on both climate change and genetic modification (McFadden, 2016). Although political ideology was not measured in our study, the average estimate of the scientific consensus on climate change in our control group (80\%) was higher than that reported for 
online samples in the US and Australia (usually in the range of 50-70\%; Cook et al., 2017; Myers et al., 2015; van der Linden, Leiserowitz, Rosenthal, et al., 2017). Similarly, the average score on the climate change acceptance scale in our control group was higher than that reported by Lewandowsky et al. for a US online sample using the same items (but with a 5- instead of 7-point Likert scale; Lewandowsky, Gignac, et al., 2013). A rough comparison can be drawn by converting averages to Percent of Maximum Possible (POMP) scores (P. Cohen, Cohen, Aiken, \& West, 1999): the average POMP score on the climate change belief scale for the control group in the current study was $74 \%$ while Lewandowsky et al. (Lewandowsky, Gignac, et al., 2013) report 54\% in their sample. Thus, we must caution the reader that the results of the current study are drawn from a sample which appears to have, on average, a higher level of climate change acceptance than seen in more representative samples.

Many of the consensus messaging studies reviewed above were undertaken using US online samples (e.g. Amazon's Mechanical Turk). The current study using a New Zealand student sample shows that the core tenet of the GBM - the mediating role of perceived scientific consensus - holds true in populations relatively different from that in which it has been most often assessed. As noted above, our sample was more accepting of the reality of human-caused climate change than participants in a similar US study. Further research with more representative or targeted samples is required to confirm that the pattern of influence outlined in the GBM remains consistent in individuals with a higher degree of scepticism regarding anthropogenic climate change. Future research should also examine the effect of 'low' consensus messages about GM food safety. Efforts in the current study were hampered by a lack of pilot data, leading to a 'low' figure which conveyed a level of consensus close to the existing estimates of the sample (i.e. did not reduce perceptions of consensus as intended). Descriptions of both a scientific consensus and lack thereof on the issue of GM food safety can be found in the mainstream media (e.g. Almendrala, 2016; Poulter, 2013). Examining the effects of such messages and how they influence beliefs will aid our understanding of how public opinion on GM food is shaped by such information.

More generally, further research into consensus messaging alone and in combination with other communications strategies, such as framing (e.g. Dixon et al., 2017), is warranted. Such studies will contribute to building an evidence-informed toolbox of communication strategies for scientists, journalists, and policymakers seeking to engage the public on the challenge of anthropogenic climate change and other publicly-debated scientific issues. 


\subsection{BRIDGING COMMENT}

Study 1 provides experimental support for the key mechanism described by the GBM: messages about scientific consensus shift perceptions of consensus which are in turn associated with personal beliefs (at least in terms of increasing acceptance of science). The experiment took place in a relatively contrived situation (an online survey) and only examined the immediate effects of a relatively blunt single sentence consensus message. Study 2 approaches the GBM using a different perspective, drawing on previously collected longitudinal data to make causal inferences about the relationship between perceptions of consensus and personal beliefs (Selig \& Little, 2012). How do these two constructs influence each other over time?

The following section presents the final version of the full manuscript published in the Journal of Environmental Psychology (Kerr \& Wilson, 2018b).

\subsection{STUDY 2: PERCEPTIONS OF SCIENTIFIC CONSENSUS DO NOT PREDICT LATER BELIEFS ABOUT THE REALITY OF CLIMATE CHANGE: A TEST OF THE GATEWAY BELIEF MODEL USING CROSS-LAGGED PANEL ANALYSIS}

\subsubsection{Abstract}

Does our perception of agreement among scientists on a scientific issue influence our own beliefs about that same issue? Recent experimental research suggests that changes in perceptions of scientific agreement play a causal role in shaping our beliefs about the existence of anthropogenic climate change. We report the results of a cross-lagged panel analysis investigating the relationship between perceived scientific consensus and personal beliefs about climate change over a five-month period in a student sample $(\mathrm{N}=356)$. We find that perception of a scientific consensus does not prospectively predict personal agreement with the reality of climate change regardless of political orientation, but personal beliefs about the reality of anthropogenic climate change prospectively predict subsequent estimates of consensus among participants with liberal voting intentions. We infer from these findings that individuals' perceptions of a consensus among scientists do not have a strong influence on their personal beliefs about climate change. 


\subsubsection{Introduction}

Many people around the world reject the reality of anthropogenic climate change (ACC), despite an estimated $97 \%$ of climate scientists agreeing that climate change is both occurring and caused by human activity (Tranter \& Booth, 2015). A lack of public certainty regarding the reality of ACC can lead to delays in action to mitigate the future effects of climate change, resulting in worse environmental and economic outcomes in the long term (Gifford, 2011; IPCC, 2014). One proposed target for increasing public acceptance of ACC is perceptions of scientific consensus (Maibach \& van der Linden, 2016). Within models of persuasion such as the elaboration likelihood model (Petty \& Cacioppo, 1986) heuristics such as consensus information ('consensus implies correctness') and source credibility ('experts can be trusted') are used to guide attitude formation. In this regard, perceptions of an expert consensus could be assumed to influence personal beliefs. In line with such assumptions, cross-sectional research has shown that perception of agreement among climate scientists is correlated with personal agreement that ACC is occurring (Ding et al., 2011; Hornsey et al., 2016).

The idea that changes in perceptions of consensus can cause changes in personal acceptance of ACC is captured in the gateway belief model (van der Linden, Leiserowitz, et al., 2015). The model posits that the perception of a consensus among scientists acts as a 'gateway cognition', a key psychological motivator causally influencing personal beliefs about the reality of ACC and related attitudes, such as support for climate mitigation policies. There is some empirical support for this model, with experiments showing that information about the scientific consensus on climate change can increase belief in ACC and that this effect is mediated by perception of scientific consensus (e.g. Brewer \& McKnight, 2017; Kerr \& Wilson, 2018a; Lewandowsky et al., 2012; van der Linden, Leiserowitz, \& Maibach, 2016). However, some studies have also reported that interventions which significantly increase perceptions of scientific agreement on climate change have no overall impact on personal beliefs (Dixon et al., 2017; van der Linden et al., 2014). In light of such results, a number of researchers have called for further research examining the causal direction of the relationship between perceived consensus and scientific beliefs (Bolsen \& Druckman, 2018a; Kohl et al., 2016; National Academies of Sciences, Engineering, and Medicine, 2016a; Pasek, 2017). If perceived consensus does not influence personal beliefs, what could explain the association between the two? It is possible that personal beliefs are casually prior to perceptions of consensus. Motivated reasoning (Kunda, 1990), biased perceptions of 
expertise (Kahan et al., 2011) and selective media exposure (Hart et al., 2009) could all skew perceptions of the scientific agreement to fit one's own beliefs. Finally, we must note that the two paths of influence are not mutually exclusive; it is possible that a bi-directional relationship exists between perceptions of consensus and personal beliefs.

Longitudinal analysis is one method which allows potential causal inferences to be made about the relationships between variables (Finkel, 1995; Selig \& Little, 2012). Autoregressive cross-lagged panel analysis examines the relationships between two or more variables across two or more time points. Although such analyses do not provide absolute 'proof' of causality, they can be a useful tool in making casual inferences given the temporal precedence of independent variables over the dependent variables. As Selig and Little (2012) note, results from panel analyses can be "used in conjunction with theory and other empirical results as one element in a larger argument in favor of a causal relationship" (p. 276).

To this end, we examine the relationship between perceptions of a scientific consensus and personal beliefs about ACC at two time points, approximately five months apart. Our first hypothesis is that self-reported beliefs and perceptions of consensus (operationalised as an estimate of the percentage of scientists in agreement with a given proposition) on this issue will be correlated at each time point, replicating the findings of previous cross-sectional research (Hornsey et al., 2016). The causal path outlined by the gateway belief model (van der Linden, Leiserowitz, et al., 2015) posits that perceptions of consensus causally influence personal agreement with the consensus. Following the model, we hypothesise that perceived consensus will prospectively predict personal agreement with the scientific consensus at a later time point.

\subsubsection{Materials and Methods}

\section{Participants}

Students undertaking consecutive first-year psychology courses at Victoria University Wellington in 2014 completed two online surveys five months apart as part of a research participation requirement $(\mathrm{N}=356 ; 21.6 \%$ men; mean age $18.64, S D=2.15)$. Only students who completed both surveys were included in analyses. Participants gave informed consent and the study was approved by the Victoria University of Wellington School of Psychology Human Ethics Committee. 


\section{Measures}

Belief in ACC was measured using a reliable four-item scale from Lewandowsky et al. (2013)(example: 'Burning of fossil fuels on the scale observed over the last 50 years has increased atmospheric temperature to an appreciable degree'; Cronbach's $\alpha$ : Time $1=.83$; Time $2=.87$ ). Responses were captured on a seven-point Likert scale (from $1=$ strongly disagree to $7=$ strongly agree) . Participants also provided estimates of the scientific consensus regarding ACC, captured by a single item: 'Out of 100 climate scientists how many do you think believe that human $\mathrm{CO} 2$ emissions cause climate change?' Participants entered a number between 0 and 100. As political orientation has been shown to be a predictor of ACC (Hornsey et al., 2016), political orientation was captured by an open-ended question asking participants which political party would vote for, with responses coded liberal or conservative (see Appendix D).

\section{Analytic procedure}

Missing data (less than $1 \%$ of values) were imputed using expectation maximization in SPSS version 23.0. A cross-lagged model was constructed using AMOS version 23.0 (Arbuckle, 2014), examining the effect of Time 1 beliefs and consensus estimates on Time 2 beliefs and consensus estimates. For the latent climate belief measure, error terms associated with each item were correlated over time. Standard errors and $95 \%$ bias-corrected confidence intervals were estimated using the AMOS bootstrapping procedure (based on 1000 iterations).

\subsubsection{Results}

The means and standard deviations for all variables and zero-order inter-correlations are displayed in Table D.2 in the supplementary material (Appendix D). Personal belief in ACC and perceptions of consensus were significantly positively correlated at both Time 1 and Time $2(r$ 's .31 and $.33, p<.001)$.

The cross-lagged model displayed a good fit to the data $\left(\chi^{2}(27)=30.94, p=.27\right.$; SRMR $=.021 ; \mathrm{CFI}=0.998 ; \mathrm{RMSEA}=.020,90 \% \mathrm{CI}[.000, .048])$. Time 1 personal belief that ACC is occurring was a significant predictor residualised of change in consensus estimates $(\beta=$ $0.20, p=.002,95 \%$ CI $[0.08,0.31])$, Time 1 consensus estimates did not significantly predict change in Time 2 belief $(\beta=0.10, p=.084,95 \%$ CI [-0.02, 0.21]; Figure 4.6). 
Constraining these cross-lagged paths to be equal significantly reduced fit $\left(\Delta \chi^{2}=12.86, p<\right.$ $.001)$, indicating the paths are different.

Time 1

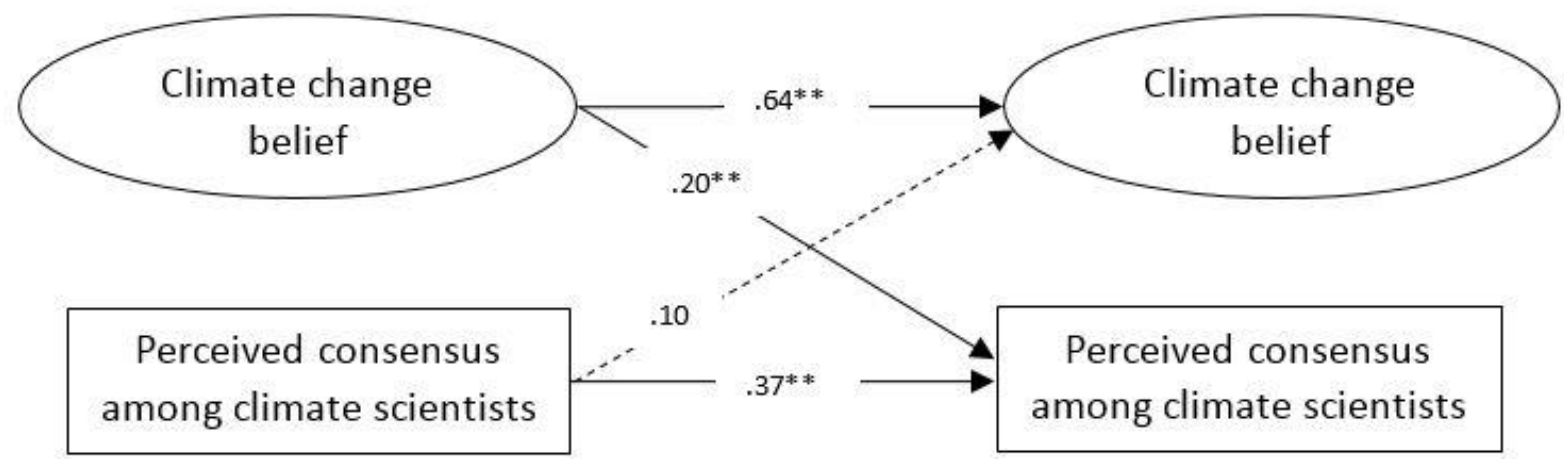

Figure 4.6. Standardized associations between personal beliefs and perceptions of consensus over time regarding the reality of ACC. Solid lines represent significant paths, and dotted lines represent non-significant paths. $* * p<0.01$

To investigate the possibility that the cross-lagged effects seen in the model are moderated by political orientation, we conducted additional nested models comparing groups defined by party voting preference (see Appendix D). The effect of Time 1 perceived consensus on Time 2 beliefs was not significant for both liberal $(N=129)$ and conservative $(N=119)$ voting groups $\left(\beta_{\text {Lib }}=0.06, p=.46,95 \%\right.$ CI $[-0.09,0.21] ; \beta_{C o n}=0.07, p=.41,95 \%$ CI $[-0.15,0.28])$. The effect of Time 1 beliefs on Time 2 perceptions of consensus did differ between groups; this path was significant for participants with liberal voting intentions but not for those with conservative voting intentions $\left(\beta_{L i b}=0.28, p>.001,95 \%\right.$ CI [0. 11, 0.45]; $\beta_{\text {Con }}=0.04, p=.66,95 \%$ CI $\left.[-0.13,0.23]\right)$.

\subsubsection{Discussion}

This study is, to our knowledge, the first to longitudinally examine the relationship between perceptions of consensus and personal beliefs regarding ACC in the absence of experimental manipulations. Consistent with prior research, and our expectations, we found that perceptions of consensus were positively correlated with personal agreement at each time point. Our analysis did not find evidence that estimates of scientific agreement at Time 1 predict personal beliefs at Time 2, thus we did not find support for our second hypothesis based on the gateway belief model. Additional analyses indicated that this effect was not moderated by party voting preference. Our results do, however, indicate that personal beliefs 
are a predictor of subsequent perceived consensus; participants' personal agreement with the reality of ACC at Time 1 prospectively predicted their estimates of scientific agreement at Time 2. However, this effect was moderated by political party support, such that personal agreement predicted later perceptions of consensus for liberal participants only. These results suggest that politically liberal individuals may be more likely to base their estimates of the scientific consensus regarding ACC on their own beliefs, rather than formulate their beliefs based on perceived agreement among scientists. This finding is discussed further in the supplementary material (Appendix D).

Our findings conflict with the results of several experimental studies showing that messages highlighting the scientific consensus increase belief in ACC, and that this effect is mediated via perceptions of consensus (e.g. van der Linden, Leiserowitz, et al., 2015; van der Linden et al., 2016). These studies indicate that in a laboratory environment participants alter their beliefs on the basis of changes in perceived consensus. Experimental research has focused on the effects of exposure to consensus messages with all other variables controlled for and over very short periods (for an exception see Deryugina \& Shurchkov, 2016). While these studies suggest that perceptions of consensus are causally prior to climate belief in an experimental setting, the current research indicates, in a more ecologically valid setting, that consensus beliefs do not have a strong influence on personal beliefs over time.

As the first study to examine the cross-lagged effects of perceived consensus and scientific beliefs over time, the current study adds to the growing body of empirical research informing the ongoing debate around efforts to communicate the scientific consensus (Cook, 2017; Kahan, 2015; Kahan \& Carpenter, 2017; Oreskes, 2017; Pearce et al., 2017b, 2017a). Acceptance of the reality of climate change is critical to the adoption of actions to mitigate and adapt to future impacts (Gifford, 2011). Drawing on the gateway belief model, some researchers have strongly endorsed the use of messages highlighting the scientific consensus on climate change to increase public acceptance of the phenomenon (Maibach \& van der Linden, 2016; van der Linden \& Lewandowsky, 2015). However, the current results, along with some recent experimental studies (e.g. Dixon et al., 2017; Kobayashi, 2018) suggest that communication campaigns seeking to increase perceptions of scientific consensus may not always have a significant effect on personal beliefs regarding ACC. Scientists, journalists and policy-makers should not simply rely solely on highlighting the expert consensus as a means to increase public acceptance of ACC. On the basis of the current preliminary research, we are hesitant to conclude that consensus messaging is totally ineffective, but encourage further 
longitudinal or field studies (Kahan \& Carpenter, 2017) with larger, more representative samples to confirm the generalisability of these findings. Researchers should also continue to explore a variety of approaches for increasing acceptance of the reality of ACC.

A key limitation of this study is the use of a student sample comprised mostly of young adults who may differ from the general public in terms of their beliefs about ACC (Milfont, Milojev, Greaves, \& Sibley, 2015) and openness to changing attitudes (Krosnick \& Alwin, 1989). This research was undertaken in a New Zealand sample which may differ from the predominantly US samples in related studies (e.g. van der Linden, Leiserowitz, et al., 2015). Although New Zealand exhibits relatively high levels of climate scepticism, climate change is less of a partisan political issue in New Zealand compared to the US (Hornsey et al., 2018a; Tranter \& Booth, 2015). Therefore, findings regarding the moderation of paths by political orientation may be different in contexts such as the US where climate change is a more politically divisive issue. The use of a single item measure to capture perceptions of consensus is a further limitation. Although variations of this single-item measure are commonly used in research examining perceptions of consensus (e.g. Lewandowsky et al., 2012; van der Linden, Leiserowitz, et al., 2015) it may have introduced measurement error into our model.

In summary, this longitudinal study captured students' agreement that ACC is occurring and their perception of agreement among the scientific community at two time points. Our analysis did not find evidence that perceptions of scientific consensus prospectively predict later personal beliefs regarding the reality of ACC, as would be predicted by the gateway belief model.

\subsection{GENERAL DISCUSSION}

Study 1 and 2 both investigated causal inferences pertinent to the relationship between perceptions of consensus and personal beliefs regarding the reality of human-caused climate change, a key pathway outlined in the GBM. In Study 1, I report experimental evidence that perception of consensus influences climate change beliefs. However, these results do not dovetail neatly with the Study 2 longitudinal findings, which suggests a reverse direction of influence: our personal beliefs colour our perceptions of scientific consensus rather than vice versa. 
There is an important postscript regarding Study 2. The Study 2 discussion acknowledged the limitations of a NZ student sample and called for further studies to confirm the findings. van der Linden, Leiserowitz, and Maibach (2018a) published a response to Kerr and Wilson (2018b) in the Journal of Environmental Psychology, presenting their own longitudinal analysis of existing panel data. The authors drew on a previous nationally representative survey of US adults ( $N=905$; Maibach et al., 2015) which collected perceptions of scientific consensus and personal agreement that human-caused climate change is occurring at two time points, five months apart. The authors undertook a crosslagged panel analysis similar to that presented in Study 2 in this thesis. Contrasting with the Study 2 results, van der Linden, Leiserowitz, and Maibach (2018a) report that perceived consensus does prospectively predict personal belief in human-caused climate change in their sample, providing support for the causal path outlined in the GBM. They also report a significant, albeit weaker, cross-lagged effect of personal belief on later perceptions of consensus (as reported in Study 2 above). Thus, in a larger and more representative (US) sample, van der Linden, Leiserowitz, \& Maibach (2018a) do find support for the GBM using longitudinal methods.

These findings suggest that the results of Study 2 may not generalise to broader samples. It is possible that the effects of perceptions of consensus on later climate change beliefs are moderated by factors such as age, gender, education and national context. For example, younger individuals may place less emphasis on scientific consensus as a basis for their own beliefs about climate change. In a review of the differences between undergraduate and general population samples, Sears (1986) writes that converging lines of evidence show "late adolescents and young adults to have more unstable, changeable, weak, and inconsistent attitudes than older adults" (p. 522). Thus, future research on the GBM should, where possible, use more generalisable adult samples. It is also possible that differences in national context underlie the difference in findings between Study 2 and van der Linden, Leiserowitz, and Maibach (2018a). For example, Salmon et al. (2017) note that news coverage of climate change in NZ tends not to focus on debate regarding the reality of climate change, therefore New Zealanders may receive less exposure to discussion of the scientific consensus in the media.

Although the results of Study 1 and Study 2 are somewhat conflicting, they agree on one finding pertinent to the rest of the thesis: perceptions of consensus do not fully explain rejection of science. For example, in Study 1, substantial shifts in perceived scientific 
consensus on climate change were linked to only small shifts in personal beliefs. In Study 2, perceived consensus and personal beliefs were only moderately correlated. What other factors can explain diverging views on issues where a scientific consensus exists?

As reviewed in Section 3.3.2, there are a number of social-psychological factors that predict rejection of science on some issues. For example, political conservatism is a wellestablished predictor of climate change scepticism and has been linked with anti-vaccination and anti-GM beliefs in some survey-based studies (e.g., Baumgaertner et al., 2018; McFadden, 2016). The following chapter examines this link in New Zealand using behavioural (as opposed to self-report) data from the social media platform Twitter. 


\section{Chapter 5: Politics and Science in the New Zealand Twittersphere}

This study explores the relationship between and political ideology and acceptance or rejection of science, using behavioural data sourced from the social media platform Twitter.

\subsection{PREFACE}

As my research journey progressed, my focus shifted away from perceptions of scientific consensus and the GBM to more distal psychological drivers of rejection of science such as political ideology (reviewed in section 3.3.2). In the process of planning out a larger survey to examine the role of ideologies in rejection of science (Studies 5 and 6), I considered alternative ways these relationships could be examined.

The inspiration for the current study came from Shi et al. (2017) who used book purchase recommendations from retail websites ('Customers who bought this item also bought...') to explore the interests of American partisans. Using several initial books as markers of political leanings, the authors scraped data from these websites to build a network of 'co-purchasing' - offering an insight into the topics that interest liberals vs conservatives. Impressed by the study's use of behavioural (rather than self-report) data, I explored ways in which I might use available social media data to examine the link between political views and science in New Zealand. ${ }^{10}$ The following study is the result of these efforts.

\subsection{STUDY 3: POLITICAL IDEOLOGY AND REJECTION OF SCIENCE ON TWITTER.}

\subsubsection{Introduction}

Several survey-based studies report an association between self-reported political ideology and rejection of science, at least at the bivariate level. That is, people who consider themselves politically conservative are more likely than their liberal counterparts to reject the mainstream scientific consensus on publicly debated issues, such as climate change, vaccination and GM food safety (Baumgaertner et al., 2018; McFadden, 2016; Rabinowitz et al., 2016). While this effect is well documented in the case of climate change denial, an

\footnotetext{
${ }^{10}$ This study was also an opportunity to develop my skills in the R programming interface.
} 
association between political ideology and beliefs about vaccine or GM food safety is less consistently reported (e.g., Drummond \& Fischhoff, 2017; Elder et al., 2018, see section 3.3.2 for further details).

Most research on rejection of science has focussed on attitudinal variables captured through surveys. However, participant responses may be biased by their perception of what beliefs are socially desirable. For example, a participant might personally believe that climate change is a scientific hoax but be aware that this is a minority position denigrated by most of their peers. As a result, they may provide a response to relevant survey questions which reflect what they think the 'right' response is, rather than what they believe. This social desirability bias has been documented in surveys focussing on environmental attitudes (Félonneau \& Becker, 2008; Milfont, 2009) and GM foods (R. B. Larson, 2018) and often raised as limitation of surveys of vaccine attitudes (Dredze, Broniatowski, Smith, \& Hilyard, 2016). The advent of social media has opened new avenues for psychological researchers to circumvent such biases by observing user behaviour directly. For example, Spiro (2016) highlights the value of using Twitter in particular to avoid social desirability bias, suggesting social media platforms are an environment "where individuals may be less likely to selfcensor their expressions" (p. 69). Dredze et al. (2016) concur, stating: "For sensitive and controversial topics, such as vaccines, asking people directly may be less accurate than observing their behaviour on social media" (p. 551).

In the following study, I work from the assumption that who a person follows on Twitter is indicative of their own attitudes and beliefs (Stroud, 2014; elaborated further below). I examine the proportion of followers of conservative (vs liberal) New Zealand political accounts who also follow accounts which promote positions counter to scientific consensus on topical scientific issues, namely climate change, GM food and vaccination. By leveraging publicly available Twitter data I can investigate the relationship between ideology and rejection of science from a novel behavioural perspective.

\section{Twitter}

The social media company Twitter was established in 2006 and describes itself as a 'microblogging platform'. Users create accounts and choose to receive information from other accounts in the form of tweets, short message comprised of up to 240 (originally 120) characters which can include links to other sites on the internet. Thus, a given account will have several followers-people who have subscribed to receive tweets-and will also follow accounts. A user's 'feed' will display tweets from all the accounts they follow, in real-time. 
User can also 're-tweet', forwarding a tweet from another account to all their followers. In this regard, Twitter can be thought of as an information-sharing network where users select which sources they want to receive (and view) information from in their Twitter feed. Twitter is an open platform; anyone can view anyone else's account profile and tweets, even if they do not follow that account. ${ }^{11}$ Twitter also provides free access to user and tweet data via its Application Programming Interface (API; Chen \& Wojcik, 2016).

\section{Twitter in psychological research}

The fact that Twitter data is free to access has made the platform a valuable and increasingly popular resource for researchers studying social interaction, social networks and the propagation of information (Kern et al., 2016). Researchers have analysed a range of types of data extracted from Twitter. Semantic analysis of the language used in tweets has offered insights into emotion (Jones, Wojcik, Sweeting, \& Silver, 2016). Examination of predictors of retweeting has shed light on which types of content are most likely to be shared (Walker, Baines, Dimitriu, \& Macdonald, 2017). Data on 'who follows who' can also provide offer useful information. Twitter users can be grouped into categories based on which accounts they follow. For example, Ritter, Preston, and Hernandez (2014) examined language differences between atheists and Christians by comparing word usage among followers of high-profile atheist and Christian accounts. Psychologists have also used Twitter follower networks to explore political ideology and its correlates. In both the US and Europe, researchers have used the following of politicians' Twitter accounts as a proxy for political partisanship and ideology (Barberá, 2015; Barberá, Jost, Nagler, Tucker, \& Bonneau, 2015; Sylwester \& Purver, 2015; S. P. Wojcik, Hovasapian, Graham, Motyl, \& Ditto, 2015). This assumption aligns with selective exposure theory which posits that people choose to consume media and information that aligns with their personal beliefs and values (Stroud, 2014). Barberá (2015) frames the decision to follow an account as incurring two types of costs, which offer an insight into user's beliefs:

If the content of the messages users are exposed to as a result of their following decisions challenges their political views, it can create cognitive dissonance. Second, given the fast-paced nature of Twitter, it also creates opportunity costs, since it reduces the likelihood of being exposed to other messages, assuming the amount of time a user

\footnotetext{
11 'Private' accounts are an exception to this. Tweets from these accounts are only visible to authorised followers. Twitter does not report on the proportion of Twitter accounts set to private but the Pew Research Centre estimates that $13 \%$ of US accounts are private (Remy, 2019).
} 
spends on Twitter is constant. In other words, these decisions provide information about how social media users decide to allocate a scarce resource - their attention. (p. 77)

Recent research confirms that following behaviour is indicative of personal attitudes, at least in the context of political ideology. Eady et al. (2019) linked survey participants' selfreported political ideology with estimates based on the political leanings of the politicians the participants follow. The authors found that self-reported conservatives are more likely to follow conservative politicians and self-reported liberals are more likely to follow liberal politicians. Golbeck and Hansen (2014) report similar results using a different approach. These authors validated their follower-based ideology estimates by manually coding a subsample of Twitter in users in their study who explicitly disclosed their voting choice in the US 2012 Presidential elections on election day (e.g., tweeting "I voted for Obama"). Users whose following behaviour suggested a conservative political stance were more likely to report voting for the Republican candidate Mitt Romney, while their liberal equivalents were more likely to report voting for the Democrat candidate Barack Obama. These studies support the assumption drawn from selective exposure theory: following behaviour on Twitter is indicative of personal attitudes.

\section{Rejection of science on Twitter}

Social media researchers have also analysed rejection of science on Twitter. For example, several studies have examined the Twitter discussion of IPCC assessment reports on climate change, analysing the content of tweets relevant to the reports, including those questioning the reality of human-caused climate change (Newman, 2017; O'Neill, Williams, Kurz, Wiersma, \& Boykoff, 2015; Veltri \& Atanasova, 2017). Mitra, Counts and Pennebaker (2016) examined the Twitter activity of several hundred pro- and anti-vaccination Twitter users, finding that conspiracy and religious-themed language was more common among antivaccination users. Whittingham, Boecker, and Grygorczyk (2019) also analysed the language of Twitter users, comparing between accounts manually coded as endorsing the view that 'GM Food Is Safe' or 'GM food is not safe.' The authors report that users who perceive GM food as risky were more likely to use language reflecting self-transcendence values and the personality trait of extraversion.

This research has focused on the content of tweets about a given scientific topic. No research, to my knowledge, has focused specifically on the followers of accounts which promote anti-scientific or science sceptical views. That is, followers of Twitter accounts 
which share content that is in opposition to the mainstream scientific consensus on publicly debated issues. This is noteworthy, as following and tweeting are two separate forms of online behaviour. People may choose to follow science-sceptical accounts, but never directly tweet about their content. The Pew Research Centre reports that $80 \%$ of all US tweets are produced by just $10 \%$ of US users, and the remaining $90 \%$ send only a median of two tweets per month (S. Wojcik, Hughes, \& Cohn, 2019). This indicates that most people on Twitter are not actively disseminating tweets, but quietly consuming. Such individuals would be invisible in the studies, such as those above, which focus specifically on tweet content. It is possible that tweeting, as opposed to following, is a more public and overt expression of one's views. Thus, tweet content-based research only examines the most extreme users, those actively engaging in spreading information and views relating to climate change, vaccines or GM food. What do we know about the people who choose to follow such users?

Following from selective exposure theory (Stroud, 2014) and Barberá's (2015) argument that account-following is a costly action, it is likely that people who follow accounts that reject the scientific consensus on a given issue are more likely to hold views similar to those promoted by such accounts. Put another way, people who follow climate sceptic accounts are likely to be sceptical about the reality of human-caused climate change, and people who follow anti-vaccination accounts are likely to have reservations about vaccines. I could use a range of labels to describe these accounts collectively, including antiscientific, science-denying, science-rejecting or contrarian accounts. For the purposes of this study, I will refer to these types of accounts as science-sceptic accounts, though I note that this terminology is debated and indeed might not exactly reflect the nature of views shared by these accounts (O’Neill \& Boykoff, 2010).

Political conservatives are more likely than their liberal counterparts to report disagreement with the scientific consensus on some issues. (Baumgaertner et al., 2018; McFadden, 2016; Rabinowitz et al., 2016). Here I analyse Twitter follower networks to determine if a similar association exists in account following behaviour. Are Twitter users who follow conservative (vs liberal) New Zealand political accounts more likely to follow accounts disseminating counter-consensus information regarding three socially debated issues: climate change, vaccination and GM food? Based on previous survey-based research I hypothesise that conservative (vs liberal) twitter users will be more likely to follow accounts rejecting the scientific consensus on climate change, vaccination and GM food. 


\subsubsection{Method}

\section{Selection of target accounts}

This study examines the intersection of two groups of Twitter users: people who follow political accounts and people who follow science-sceptical accounts. It is important to be clear how these initial accounts are selected. Although the terms user and account are interchangeable in the context of Twitter, for clarity I will use the term account to refer to political and science sceptical accounts, and the term user to refer to the 'everyday' people who follow these accounts. Some studies which categorise users by who they follow offer little justification for their selection of target accounts. For example, Ritter, Preston, and Hernandez (2014) categorise Twitter users into Christians and atheists based their following of accounts such as @ Pontifex (Pope Francis) and @RichardDawkin (prominent atheist Richards Dawkins), with no discussion of why these accounts were chosen over other possible accounts. ${ }^{12}$ Here, I offer further detail on how political and science-sceptical accounts were selected.

Political accounts. Selection of political accounts is relatively straightforward. In the current study, I use official political party accounts of all parties represented in the current parliament and all party-affiliated Members of Parliament (MPs) in the New Zealand house of representatives at the time of data collection. A full list of all MPs' Twitter accounts is curated by the New Zealand parliament official Twitter account (@NZParliament; Twitter.com/NZParliament/lists/mps). In the New Zealand political context, the Labour and Green parties are considered politically liberal, while the National and ACT parties are considered relatively conservative (Jahn, Düpont, \& Rachuj, 2018). The fifth party represented in parliament, NZ First, is less clearly defined on the liberal-conservative spectrum. NZ First holds typically conservative views on issues such as taxation and immigration (New Zealand First, n.d.). However, though having previously entered in coalition with the National Party, as of 2017 the party is in Government in coalition with the left political bloc of the Labour and Greens parties. As such, we exclude NZ First from the main analysis here, however, it is included in a supplementary analysis examining individual parties (Appendix E).

Using the following of political accounts as a proxy for ideology, I classify liberal Twitter users as those who follow either Labour or Green party and MP accounts.

\footnotetext{
12 The “@” prefix denotes a specific user account on Twitter.
} 
Conservative Twitter users are those who follow either National or ACT party and MP accounts. Users who follow both sets of accounts are excluded as these are people who likely take a general interest in politics rather than leaning to one end or the other of the political spectrum (these users are included in an alternative analyses section in the results).

Science-sceptic accounts. There are several methods that could be used to identify science-sceptic accounts. For example, some analyses have collected millions of tweets on a given topic and analysed the network of retweeting (i.e. who shares information from whom) to identify clusters of users that appear to share a common position on the topic. Such analyses require considerable computational power and access to a large amount of Twitter data via the 'Twitter firehose' - a paid-for service allowing full access to all tweets over a given study period. I took a more pragmatic approach for the current study, piggybacking of the results of such network analyses. Specifically, I drew on analyses which identified specific, influential accounts representing the counter-scientific position on three different issues: climate change (Carbon Brief, 2016), GM food safety (Fields, 2015; Mishra, 2017), and vaccination (Diresta \& Lotan, 2016). I must note that these analyses were published online and were not subject to peer review. I also searched the peer-reviewed literature for references to high-profile science-sceptical Twitter accounts. Details of specific accounts clearly defined as opposing mainstream science were scarce, though some sources offered an indication of individuals and groups with an online presence who could then be searched for on Twitter platform (e.g. Lewandowsky, Oberauer, et al., 2013, supplementary material).

This approach yielded several accounts for each topic, which I will refer to as the initial sets. To increase the number of science-sceptical accounts (and thus potential overlap of followers with followers of political accounts), I also performed a secondary analysis of the initial sets examining who these accounts followed to identify further accounts that may have been missed or gained recent prominence. For example, if a given account is followed by most of the climate sceptic accounts in the initial set, it is quite likely to share climate sceptic content. Here I used an arbitrary cut-off to limit further additions; if an account was followed by more than seven (climate change) or four (vaccination and GM food) accounts in the initial set, it was added to the science-sceptical group of accounts for a topic. ${ }^{13}$ Specifically, user IDs of all accounts followed by the initial sets of climate, GM and vaccine sceptic

\footnotetext{
${ }^{13}$ The differing criterions for climate vs GM food and vaccination accounts is due to a larger initial set of climate sceptic accounts.
} 
accounts were extracted from Twitter via the Twitter API using the rtweet package in R (Kearney, 2019). User IDs which appeared in seven/four or more of the lists were added to the science-sceptic lists.

As a final check, all accounts were subject to several inclusion criteria. Only accounts which had more than 2000 followers were included in the final lists (median $=6,486$ followers; range $=2,004-171,123$ followers). To ensure that accounts were posting relevant content, the 100 most recent tweets from each account were extracted via the API and automatically coded as relevant based on the presence of keyword strings (see Table 5.1 for criteria). Only accounts with a minimum of $30 \%$ topic relevant recent tweets were retained. Finally, account profiles and the most recent 100 tweets from retained accounts were reviewed to ensure that the content broadly reflected a position in disagreement with the scientific consensus position i.e. that human-caused climate change is happening, that GM food is safe to eat, or that vaccines are safe and effective (two accounts were removed from the climate sceptic list as these were high-profile academics who support the scientific consensus on climate change).

Table 5.1

Inclusion search terms for coding tweet relevance

\begin{tabular}{ll}
\hline Topic & Search string \\
\hline Climate change & clim|warm|co2|carbon|fossil|temp \\
Vaccination & vax|vacc|injury|immu|autis \\
GM food & gm|gene|biotec|monsanto \\
\hline
\end{tabular}

Note: the bar symbol 'l' is equivalent to the 'OR' Boolean operator.

To summarise, the final lists of science sceptical accounts represented either Twitter accounts previously identified as part of science sceptic networks or relevant accounts followed by those accounts. All accounts in the final lists had a sizable following (2000 or more followers) and more than $30 \%$ of their recent tweets related to the core topic. ${ }^{14}$ The

\footnotetext{
${ }^{14}$ Climate sceptic accounts were subject to an additional check. The website desmogblog.com maintains a database of climate sceptic individuals and organisations including examples of public statements questioning human-caused climate change. Of the 24 climate accounts included in the study, 16 (67\%) of the individuals and organisations appear in this database. Unfortunately, similar databases were not available for vaccine and GM opponents.
} 
names and description of accounts included in each group are reported in Tables E.1-3 (Appendix F).

\section{Collection of follower user IDs}

The study was approved by the Victoria University of Wellington School of Psychology Human Ethics Committee. To identify the proportion of liberal and conservative account followers who also followed science sceptic accounts, lists of followers' user IDs were extracted from Twitter via the Twitter API using the rtweet package in R. ${ }^{15}$ Lists were combined to create groups of users categorised by the accounts they followed: liberal NZ users, conservative NZ users, climate sceptical users, GM sceptical users and vaccine sceptical users.

\subsubsection{Results}

Table 5.2 details the total number of target accounts in each group and the number of unique users who follow accounts in each group. There were far more unique followers of liberal accounts than conservative accounts, consistent with overseas research (Sloan, 2017; S. Wojcik, Hughes, et al., 2019).

Table 5.2

Number of accounts in, and unique followers of each list used in the study.

\begin{tabular}{lll}
\hline List & $\begin{array}{l}\text { Total accounts } \\
\text { in list }\end{array}$ & $\begin{array}{l}\text { Total number of } \\
\text { unique followers }\end{array}$ \\
\hline Liberal (Labour/Greens) political accounts & 53 & 457,547 \\
Conservative (National/ACT) political accounts & 48 & 33,584 \\
Climate sceptic accounts & 24 & 214,187 \\
Vaccine sceptic accounts & 39 & 189,795 \\
GM sceptic accounts & 31 & 411,202 \\
\hline
\end{tabular}

Note. Followers of both liberal and conservative $(N=37,918)$ accounts are excluded.

\footnotetext{
${ }^{15}$ A substantial number of users follow the Twitter account of Prime Minister Jacinda Ardern $(\mathrm{N}=409,356)$. It is possible that users may follow her account due to a general interest in New Zealand and politics rather than an interest in the politics of the Labour party per se. All analyses were repeated with followers of Jacinda Ardern only removed from the dataset. The pattern of results was identical although effect sizes were slightly attenuated.
} 
Table 5.3 presents three $2 \times 2$ contingency tables detailing the absolute and proportional overlap between followers of political accounts and followers of climate, of vaccine or GM sceptic accounts. As can be seen in the table, $0.51 \%$ of liberal account followers and $3.47 \%$ of conservative accounts followers also follow climate sceptic accounts. A chi-square test of independence was performed to examine the relationship between following liberal (vs conservative) accounts and following climate sceptic accounts. The relationship between these variables was significant, $\chi^{2}(1)=3891.5, p<.001, \varphi=.09$. Conservative account followers were more likely to follow climate sceptic accounts than liberal account followers, though the measure of effect size, $\varphi$, indicates this effect is 'small'. We see a similar pattern with followers of vaccine sceptic Twitter accounts. A greater proportion of conservative account followers also follow vaccine sceptic accounts $(2.75 \%)$, compared to liberal account followers $(0.33 \%), \chi^{2}(1)=3722.3, p<.001, \varphi=.09$. In the case of GM sceptic accounts, we also find that a greater proportion of conservatives (2.37\%) follow these accounts compared to liberals $(0.91 \%), \chi^{2}(1)=666.68, p<.001, \varphi=.04$. The results across the three different topics examined are summarised in Figure 5.1.

Table 5.3

Number and percentage of liberal and conservative account followers who do or do not follow science sceptic accounts.

\begin{tabular}{|c|c|c|c|c|c|c|}
\hline \multirow[b]{2}{*}{$\begin{array}{l}\text { Political } \\
\text { group }\end{array}$} & \multicolumn{2}{|c|}{$\begin{array}{l}\text { Climate sceptic } \\
\text { accounts }\end{array}$} & \multicolumn{2}{|c|}{$\begin{array}{c}\text { Vaccine sceptic } \\
\text { accounts }\end{array}$} & \multicolumn{2}{|c|}{ GM sceptic accounts } \\
\hline & Follows & $\begin{array}{l}\text { Does not } \\
\text { follow }\end{array}$ & Follows & $\begin{array}{l}\text { Does not } \\
\text { follow }\end{array}$ & Follows & $\begin{array}{c}\text { Does not } \\
\text { follow }\end{array}$ \\
\hline \multirow[t]{2}{*}{ Liberal } & 2,320 & 455,227 & 1,510 & 456,037 & 4169 & 453,378 \\
\hline & $(0.51 \%)$ & $(99.49 \%)$ & $(0.33 \%)$ & $(99.67 \%)$ & $(0.91 \%)$ & $(99.09 \%)$ \\
\hline \multirow[t]{2}{*}{ Conservative } & 1,165 & 32,419 & 925 & 32,659 & 797 & 32,787 \\
\hline & $(3.47 \%)$ & $(96.53 \%)$ & $(2.75 \%)$ & $(97.25 \%)$ & $(2.37 \%)$ & $(97.63 \%)$ \\
\hline
\end{tabular}




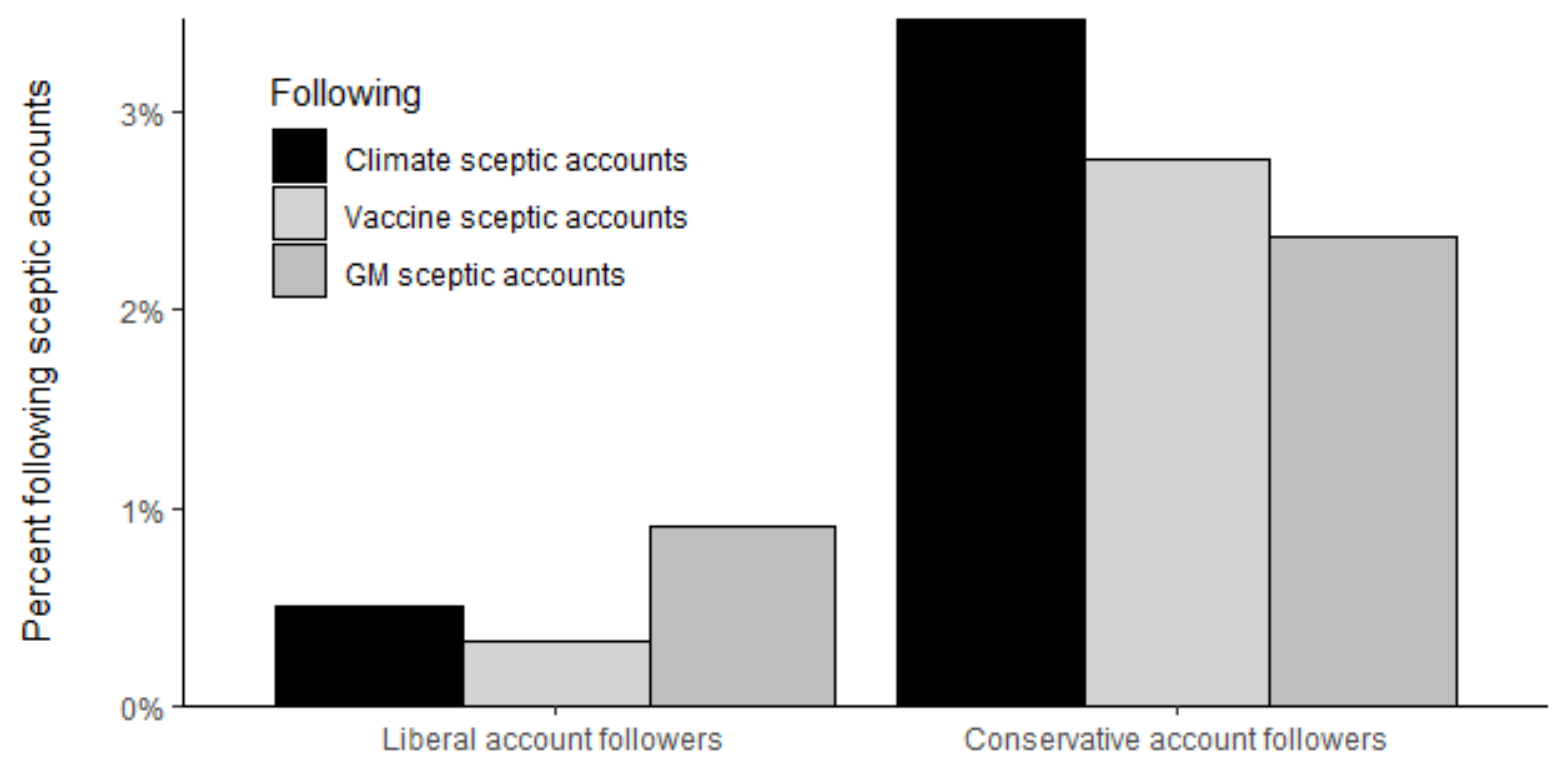

Figure 5.1. Percentage of NZ political Twitter account followers who also follow science sceptic accounts.

I also examined the overlap between followers of New Zealand political accounts and science sceptic accounts at the party level (see Appendix E). Results were broadly similar although I identified followers of the Green party and its MPs as having the highest proportion of GM sceptic account followers, in contrast to the main analysis.

\section{Alternative analyses}

The previous analyses examine the overlap between groups defined by their following of political or science sceptic accounts. So far, I have treated liberal and conservative followers as two groups, when in fact there are two parties in each group, which do differ in terms of placement on the liberal-conservative spectrum. I also exclude followers of both liberal and conservative accounts. In this section, I use the method described by Golbeck and Hansen (2014) to create an alternative, continuous measure of political ideology which takes into account ideological differences between parties as well as followers of multiple parties (see also, Boutyline \& Willer, 2017). These authors estimate the political ideology of US Twitter users based on which members of the US Congress they follow, incorporating independent measures of each congress members' ideological leanings. In the case of Golbeck and Hansen, each congressperson was assigned an ideology value based on DWNOMINATE scores (dynamic, weighted nominal three-step estimation). DW-NOMINATE scores are based on members' voting history in congress and broadly represent the liberal- 
conservative spectrum (Poole \& Rosenthal, 2001). Golbeck and Hansen (2014) calculated a political ideology index, $p$-score, for all Twitter users who follow at least one member of Congress on Twitter. This p-score was the average of all the DW-NOMINATE scores of congress members a user followed.

In the New Zealand political context, we do not have equivalent individual-level data for MPs. However, there are estimates of political ideology at the party level. Jahn, Düpont, and Rachuj (2018) used a deductive and inductive reasoning approach to estimate each New Zealand political party's ideological placement on a left-right spectrum. These scores are based on the coding of statements in political party manifestos, drawing on Noberto Bobbio's (1996) theoretical work on the distinction between the political Left and Right. Jahn, Düpont, and Rachuj's (2018) estimates of New Zealand party positions on the left-right scale are shown in Table 5.4. Incorporating these scores in the approach of Golbeck and Hansen (2014), we can calculate a rough $p$-score for individuals in the current dataset. Each political account is assigned a left-right value based on party, drawn from Jahn, Düpont, and Rachuj (2018). I then calculate a users' p-score as the average of all the left-right values of the accounts they follow $(N=533,517, M=-4.38, S D=4.22)$.

Table 5.4

Left-right scores for New Zealand political parties based on Jahn, Düpont, and Rachuj's (2018) analysis.

\begin{tabular}{ll}
\hline Party & Left-Right score \\
\hline Greens & -14.15 \\
Labour & -4.83 \\
New Zealand First & -1.17 \\
National & 7.20 \\
ACT & 22.35 \\
\hline
\end{tabular}

Considering our corpus of all Twitter users who follow NZ political accounts, we can compare the p-scores of users who do and do not follow science sceptic accounts. A Welch two-sample t-test indicated that followers of climate sceptic accounts had a significantly higher (i.e. more conservative) mean p-score than non-followers of these accounts, $t(4283.9)$ 
$=-30.20, p<.001$, Cohen's $d=-0.85$. This was also the case for followers (vs. nonfollowers) of vaccine sceptic accounts, $t(2757.7)=-23.55, p<.001, d=-0.77$. In both cases Cohen's $d$ values indicate a relatively large effect size (J. Cohen, 1988). In contrast, followers of GM sceptic accounts had, on average, a slightly lower (i.e. more liberal) p-score than nonfollowers, $t(5519)=-3.43, p<.001, d=-0.07$, though the effect size is very small. Mean $\mathrm{p}$ scores for each group are shown in Figure 5.2. The results indicate that, among people who follow New Zealand political accounts, those who also follow climate or vaccine sceptic accounts tend follow, on average, more conservative politicians and parties. Those who follow GM sceptic accounts follow, on average, more liberal politicians and parties, though this effect is very small.

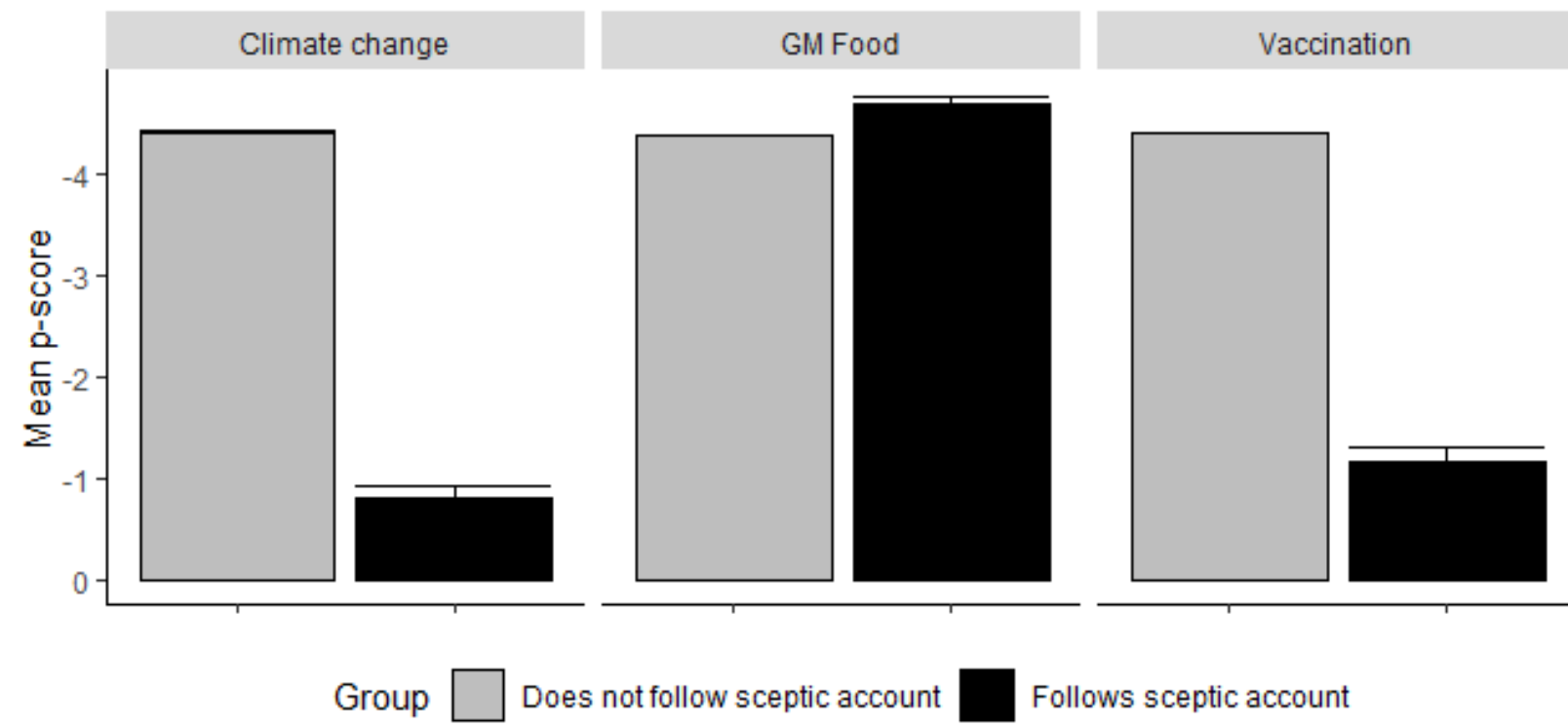

Figure 5.2. Average p-score for followers and non-followers of science sceptic accounts. Note the $\mathrm{Y}$-axis has been reversed for readability (lower numbers indicate a more liberal estimated ideology). Error bars represent the standard error of the mean.

I also calculate the correlation between each p-score and the number of science sceptic accounts followed (for all users). As the data are non-normal, I use Spearman's rank-order correlation (Field, 2009). The p-score positively correlated with the number of climate sceptic accounts followed, $r_{s}=.06, p<.001$, and the number of vaccine sceptic accounts followed, $r_{s}$ $=.04, p<.001$. In the case of GM sceptic accounts, $\mathrm{p}$-score was negatively correlated with the number of accounts followed, $r_{s}=-.01, p<.001$. The results indicate that, among Twitter users who follow NZ political parties and MPs, those whose following behaviour indicates a 
more conservative political ideology are more likely to follow a greater number of Twitter accounts sceptical of climate change and vaccine safety. In the case of GM sceptical accounts, we again see a (very) small negative effect of ideology such that left-leaning users are more likely to follow a greater number of GM sceptic accounts.

\subsubsection{Discussion}

Are conservatives more likely to reject science? This study used the following of relevant Twitter accounts as proxy for political ideology and beliefs about scientific issues. Of the entirety of Twitter users who follow New Zealand political accounts, very few followed any of the science sceptic accounts included in this study. However, we do find that a greater proportion of conservative (vs. liberal) New Zealand Twitter users follow science sceptical accounts. Put another way, Twitter users who are interested in content from conservative parties and politicians are more likely than their liberal counterparts to be interested in content from individuals and organisations that disagree with the scientific consensus on climate change, GM food and vaccines. However, analyses using alternative approaches to estimating users' political ideology presented inconsistent results regarding the following of GM sceptic accounts. These results suggest that conservatives may be less likely to follow anti-GM accounts. Though the effect is statistically significant, the practical significance of this relationship is questionable. Examination of following at the party level (Appendix E) indicates that followers of the Green party and its MPs were more likely to follow GM-sceptic accounts than followers of other parties. This effect appears to have been masked in the main analysis due to the large number of Labour party followers in the 'liberal' grouping. This result is somewhat unsurprising given the Green party's longstanding GM policy position on prohibiting "genetically modified and transgenic organisms that are intended for release into the environment or food chain" (Green Party of Aotearoa New Zealand, n.d.). This exception illustrates the fact that many factors, including partisanship, not just broad left-right political orientation, play a role in rejection of science.

While the absolute numbers of users following both political and science-sceptical accounts were relatively small, we can assume that the science sceptic accounts selected represent only a small slice of the total number of science sceptic accounts on the Twitter platform. Proportions would be higher if the analysis were able to cover, say, all climate sceptic accounts on Twitter. Furthermore, following an account is a very concrete action and represents not merely an interest in the content, but an active desire to receive more information going forward. Thus, followers of political accounts are likely more political than 
the average New Zealander (this is reflected in US analyses; S. Wojcik, Huges, \& Remy, 2019) and followers of science sceptic accounts represent those at the extreme end of the spectrum of science rejection on a given issue. In this sense, the users identified as following both political and science sceptic accounts could be considered the 'tip of the iceberg'.

This analysis reinforces the findings of survey-based research with behavioural data. Several studies report a positive bivariate correlation between political conservatism and rejection of science on these issues (e.g. Baumgaertner et al., 2018; McFadden, 2016). I would stress at this point that it is unlikely that political conservatism per se is driving rejection of science across all issues in this context. The relationship between climate scepticism and conservatism is well documented in New Zealand and overseas (Hornsey et al., 2016; McCright \& Dunlap, 2011; Milfont et al., 2015). However, previous research noting correlations between conservatism and rejection of science in other domains has also used SEM or regression-based modelling to show that other factors, such as religiosity or conspiracy beliefs, may underlie the observed association (Lewandowsky, Gignac, et al., 2013; Rutjens et al., 2017). For example, Rutjens et al. (2017) report political conservatism is not a predictor of anti-vaccine beliefs once religiosity is considered. I was unable to control for these variables in the current study. Future research applying this study's methodology could also incorporate the following of other types of accounts to capture such variables. For example, the following of religious or conspiracy focused twitter accounts could be used as proxies for religiosity and conspiracy thinking respectively and incorporated in multivariate analyses predicting the following science sceptic accounts.

There are several limitations to this study. Firstly, Twitter users are not representative of the wider population. Research from the UK and US indicates that Twitter users tend to be younger and left-leaning politically (Sloan, 2017; S. Wojcik, Hughes, et al., 2019). This political skew was apparent in our corpus of users, with liberal account followers outnumbering conservatives by a factor of 10 . However, I was still able to collate a list of more than 30,000 Twitter users who follow conservative (but not liberal) New Zealand political accounts. My approach to selecting science-sceptic accounts was not entirely systematic, drawing on non-peer-reviewed analyses to identify accounts which oppose the scientific consensus. While I did take steps to expand the number of accounts considered and ensure they were relevant, it is possible that there is some bias in the selection of accounts. I have assumed that the users covered in this study are New Zealanders, but there will be users from outside New Zealand who have been captured in this dataset. User location can be 
ascertained from tweet data if permitted by the user. However, Graham, Hale, and Gaffney (2014) report that less than $1 \%$ of tweets carry this geolocation information. Future research could take advantage of emerging neural network approaches to estimate user location based on tweet data (Huang \& Carley, 2017). Finally, I must acknowledge that following sciencesceptic accounts may not reflect an individual's personal views. Some people may follow sceptics because of other, non-science related content these accounts share, or because they are interested in alternative viewpoints to their own. Future research should also seek to combine twitter and survey data to investigate the extent to which following behaviour is linked to self-reported acceptance or rejection of science. Despite these limitations, the study offers some ecologically valid support for the established link between self-reported political ideology and rejection of climate and vaccine science. This is the first study to examine the overlap in the follower networks of political accounts and science sceptical accounts. This approach, or more nuanced versions of it, could also be applied to other national contexts where politicians have a presence on Twitter (e.g. the US, UK, Australia).

Overseas research on the link between political ideology and rejection of science has highlighted the role of trust in scientists. Conservatives are broadly less trusting of scientists while liberals are more trusting (Hartman et al., 2017; McCright, Dentzman, Charters, \& Dietz, 2013). Thus, we might also expect that conservatives are less likely to follow high profile 'pro-science' and science celebrity accounts (such as 'I Fucking Love Science', Neil DeGrasse Tyson and Bill Nye). Research investigating these relationships would offer further insight into the link between political ideology and general evaluations of science.

These findings do raise some concerns about the spread of counter-scientific information on social media. People who choose to follow science sceptic accounts may find their opinions reinforced, and therefore develop a skewed perception of the prevalence of their views (Boyd, 2019). Preventing the dissemination of such information poses a challenge-many of the sceptic accounts are real people expressing their personal beliefs, and simply removing such accounts has implications regarding censorship and free speech (B. Martin, 2015; Mortimer, 2017; York, Greene, \& Gebhart, 2019). ${ }^{16}$ Lewandowsky, Ecker, and Cook (2017) offer a series of recommendations for improving the quality of online information, including: educating the public in 'information literacy', social media platforms

\footnotetext{
${ }^{16}$ It is worth noting that one of the anti-vaccination accounts included in this study (@ ViraBurnayeva; full name "LotusOak") has been speculated to be an automated bot sharing vaccine misinformation (Menczer \& Hui, 2019).
} 
introducing fact-checking algorithms, and developing 'trust badges' or a quality rating system for information shared online. Twitter has already taken some steps to counter vaccine misinformation. In May 2019, the platform announced that US users who search for vaccinerelated information on Twitter will see a message and 'pinned' tweet at the top of search results, directing users to information from the US Department of Health and Human Services (Harvey, 2019). Such interventions could also be implemented for other controversial issues such as climate change, although the politicised nature of some issues may hinder efforts in this space (De Pryck \& Gemenne, 2017).

To summarise, this study examined followers of political Twitter accounts in New Zealand and followers of accounts that take a stance against mainstream science on climate change, GM food and vaccines. I found that Twitter users who follow conservative political accounts were more likely than people who follow liberal political accounts to also follow science sceptical accounts (though the association with anti-GM accounts is not consistent across analytical approaches). This research, based on behavioural data, reinforces the links between political ideology and science beliefs found in some survey-based research: political conservatives, broadly, are more likely to hold beliefs in opposition to established scientific consensus on climate change and vaccination (Baumgaertner et al., 2018; Hornsey et al., 2016; Rabinowitz et al., 2016). However, as reviewed in Section 3.3.2, political ideology is just one of a number of interrelated 'attitude roots' associated with rejection of science. The following chapter aims to identify the relative contribution of each of these roots to rejection of science across a range of issues and place predictors within the larger theoretical framework of the DPM. 



\section{Chapter 6: The Role of RWA and SDO in Rejection of Science}

\subsection{PREFACE}

As my thesis research progressed my focus shifted towards investigating the ideological drivers of rejection of science. I set about planning larger surveys to examine the relative strength of various ideological factors in predicting rejection of science across a wider range of issues than considered in previous studies (e.g., Lewandowsky, Gignac, et al., 2013; Rutjens et al., 2017).

At this time, I also became more familiar with the DPM thanks to the work of a fellow $\mathrm{PhD}$ student, Samantha Stanley. I was surprised to learn that the DPM's central constructs, RWA and SDO, predicted many of the ideological factors associated with rejection of science (reviewed in section 3.3.3). A relatively recent flurry of papers had linked both RWA and SDO to climate change denial (reviewed in Stanley \& Wilson, 2019), but the role of these attitudes in other scientific debates (e.g. vaccination) remained wholly unexamined. This glaring gap in the literature presented an excellent opportunity to place the previously researched attitude roots of rejection of science in a wider social psychological framework. Threfore, I decided to use RWA and SDO as the starting point for examining the ideological drivers of rejection of science.

This chapter starts with a preliminary study examining the associations between the RWA, SDO and rejection of science in a student sample. I then introduce more complex models examining a range of potential mediators of these effects. Here I examine the effects of these constructs on beliefs about specific issues (e.g. climate change) as well as on a broader, second-order variable capturing a generalised acceptance of scientific findings. Taking on board lessons learned from my earlier studies, I survey larger, non-student samples and compare results between US and New Zealand samples.

\subsection{STUDY 4: DO IDEOLOGICAL ATTITUDES PREDICT REJECTION OF SCIENCE? A STUDY OF NEW ZEALAND STUDENTS.}

\subsubsection{Introduction}

This study presents a preliminary investigation of the relationship between two 'ideological attitudes'—RWA and SDO — and rejection of science in a NZ student sample. 
Firstly, I seek to replicate the finding that both RWA and SDO predict rejection of climate science (Stanley \& Wilson, 2019). Secondly, I examine the extent to which these attitudes predict rejection of science in two other domains: GM food and vaccination.

\subsubsection{Method}

\section{Participants}

Participants in the study were students enrolled in an introductory psychology course at Victoria University of Wellington in 2017. A total of 547 participants took part in the study (415 women, 128 men and four reporting non-binary or no gender). Ages ranged from 16 to 64 years of age $(M=19.19, S D=3.56)$.

\section{Materials}

The following measures were embedded in a larger omnibus survey covering a wide range of topics. Responses to all items were captured on a 7-point Likert scale ranging from 1 (Strongly disagree) to 7 (Strongly agree). All scales demonstrated satisfactory reliability (see Table 6.1).

Participants completed Altemeyer's (1996) 30-item RWA scale (example item: Obedience and respect for authority are the most important virtues children should learn) and the 16-item $\mathrm{SDO}_{7}$ scale (Ho et al., 2015) (example: We shouldn't try to guarantee that every group has the same quality of life). Rejection of science dependent variables included Lewandowsky et al.'s (2013) 5-item scale capturing belief that human activity is causing climate change (example: Human CO2 emissions cause climate change), Dixons'(2016) 3item GM food safety scale (example: GM food is safe to eat). A single item measured acceptance of vaccine safety: Vaccines are safe. Responses were coded such that higher values indicated greater agreement with the scientific consensus position.

\section{Procedure}

Participants completed the survey online on the SurveyMonkey platform in return for partial fulfilment of a mandatory course research component. Participants provided informed consent and were fully debriefed at the end of the survey. The study was approved by the Victoria University of Wellington School of Psychology Human Ethics Committee (application number: 0000023961). 


\subsubsection{Results}

Study 4 descriptive statistics and bivariate correlations are shown in Table 6.1. RWA and SDO were positively correlated $(r=.69, p<.001)$. RWA was negatively correlated with climate change belief ( $r=-.52)$, GM food safety beliefs $(r=-.26)$ and vaccine safety beliefs $(\mathrm{r}=-.32)$, as was SDO ( $r \mathrm{~s}-.48,-.23$ and -.28 respectively; all $p<.001)$.

Table 6.1

Study 4 descriptive statistics and correlations

\begin{tabular}{llllllll}
\hline & $\mathrm{M}$ & $\mathrm{SD}$ & $\alpha$ & 1 & 2 & 3 & 4 \\
\hline 1. RWA & 2.64 & 0.89 & .95 & & & & \\
2. SDO & 2.81 & 0.89 & .90 & $\mathbf{. 6 9}$ & & & \\
3. Climate & 5.59 & 0.91 & .75 & $\mathbf{- . 5 2}$ & $\mathbf{- . 4 8}$ & & \\
4. GM Food & 4.22 & 1.21 & .86 & $\mathbf{- . 2 6}$ & $\mathbf{- . 2 3}$ & $\mathbf{. 2 5}$ & \\
5. Vaccines & 5.69 & 1.27 & - & $\mathbf{- . 3 2}$ & $\mathbf{- . 2 8}$ & $\mathbf{. 3 4}$ & $\mathbf{. 4 3}$ \\
\hline
\end{tabular}

All correlations significant $(p<.001)$

The variables 'Climate', 'Genetic modification', and 'Vaccination' reflect (respectively) agreement that: human activities cause climate change, GM foods are safe to eat, and vaccines are safe.

To determine the independent effects of ideological attitudes on scientific beliefs I regressed each of the three rejection of science variables onto RWA and SDO. As previous research has shown age and gender influence beliefs about scientific issues, I also include these demographic variables as covariates (Elder et al., 2018; McCright \& Dunlap, 2011). All regressions were significant $(F \mathrm{~s}(4,538)>16.90, p$ 's $<.001)$. As seen in Table 6.2, both RWA and SDO are negative predictors of agreement that: anthropogenic climate change is occurring $\left(\beta_{\mathrm{RWA}}=-.36, p<.001 ; \beta_{\mathrm{SDO}}=-.24, p<.001\right)$, GM food is safe to eat $\left(\beta_{\mathrm{RWA}}=-.20, p\right.$ $\left.<.001 ; \beta_{\mathrm{SDO}}=-.12, p<.05\right)$, and vaccines are safe $\left(\beta_{\mathrm{RWA}}=-.24, p<.001 ; \beta_{\mathrm{SDO}}=-.12, p<\right.$ $.05)$. 
Table 6.2

Demographic and ideological predictors (correlates) of acceptance of science.

\begin{tabular}{llll}
\hline & Climate change & GM food & Vaccines \\
\hline Age & -.02 & $-.11 * *$ & -.04 \\
Gender $(\mathrm{M}=1)$ & .07 & $.19 * * *$ & .07 \\
RWA & $-.36 * * *$ & $-.20^{* * *}$ & $-.24 * * *$ \\
SDO & $-.24 * * *$ & $-.13 *$ & $-.12 *$ \\
Adjusted $R^{2}$ & $.29 * * *$ & $.12 * * *$ & $.11 * * *$ \\
\hline$p<.05, * *<.01 * * * p<.001$. & &
\end{tabular}

\subsubsection{Bridging comment}

Study 4 confirms that in this student sample, RWA and SDO play a role in (or reflect) rejection of science, and this extends beyond climate change - the issue most commonly associated with these ideological constructs. However, Study 4 did not offer any insight into the reasons why individuals who are more authoritarian or socially dominant are more likely to reject specific scientific claims. This study also used a student sample, which may limit the generalisability of the results to the wider population.

The following studies investigate the effects of RWA and SDO on a wider range of rejection of science outcomes, in US and NZ adult samples. These studies also investigate the extent to which a range of ideological and science-related beliefs explain the links between RWA, SDO, and rejection of science.

\subsection{STUDY 5: INVESTIGATING THE RELATIONSHIP BETWEEN IDEOLOGICAL ATTITUDES AND REJECTION OF SCIENCE IN AN ONLINE US SAMPLE}

\subsubsection{Introduction}

Study 5 seeks to identify the ideological and science-specific factors which mediate the effects of ideological attitudes on rejection of science. I include four ideological constructs which are associated with rejection of science in some domains and predicted by ideological attitudes (reviewed in Sections 3.3.2 and 3.3.3): political ideology, free market ideology, religiosity and conspiracy mentality (Hornsey \& Fielding, 2017; Lewandowsky, Gignac, et al., 2013; Rutjens et al., 2017). I also include two science-specific mediators known to positively predict acceptance of scientific claims: a brief measure of general science 
knowledge and perceived credibility of scientists. (Drummond \& Fischhoff, 2017; Hartman et al., 2017).

In this study (and Study 6) I first regress rejection of science variables onto RWA, SDO and demographic controls, as in Study 4. Next, to examine potential mediators I use SEM to estimate the associations between latent constructs. This approach has advantages over regression-based analyses in that it accounts for measurement error and allows estimation of multiple indirect (i.e. mediated) effects (Gana \& Broc, 2018). The use of the R-based SEM package lavaan (Rosseel, 2012) also allows the use of estimators which account for nonnormal data.

\subsubsection{Methods}

\section{Participants}

Participants were recruited via the Prolific Academic platform (prolific.ac), with participation limited to only US-based individuals. A total of 710 individuals completed the survey. Participants who failed either of two attention checks (instructed-response items, e.g. 'Please select "agree” for this statement'; Gummer, Roßmann, \& Silber, 2018) embedded in the survey were removed from the sample (47 participants).

There was a relatively even split of gender in the remaining sample with 325 women (49.0\%), 316 men $(47.6 \%)$ and 22 participants reporting no or non-binary gender $(3.3 \%$; excluded from analyses including gender as a covariate). Age was captured on a twelve-point scale, with 25-29 years the median and modal response. Most participants identified as white (76.3\%), followed by black/African American (10.4\%), Asian (10.3\%), Hispanic/Latino $(3.8 \%)$ and other $(3.7 \%)$.

\section{Materials}

In discussing the variables examined in this study I differentiate between the ideological attitudes RWA and SDO and other ideological constructs (religiosity, conservatism, free market ideology and conspiracy mentality) which I will refer to as ideological mediators, given their role in the current study and their more theoretically proximal role as foundations for issue-based attitudes. I refer to science literacy and perceived credibility of scientists as science-specific mediators and the dependent variables capturing agreement with the scientific consensus are collectively referred to as rejection of science variables, although higher scores indicate greater agreement with the consensus position (Lewandowsky, Gignac, et al., 2013; Rutjens et al., 2017). Unless otherwise 
specified, all responses were recorded on a 7-point Likert scale (Strongly disagree to Strongly agree). With one exception, noted below, all scales demonstrated satisfactory reliability (see Table 6.3)

Ideological attitudes. Participants completed Duckitt, Bizumic, Krauss, and Heled's (2010) 18-item revised form of Altemeyer's (1996) RWA scale (example item: Obedience and respect for authority are the most important virtues children should learn) and the short 8-item $\mathrm{SDO}_{7}$ scale (Ho et al., 2015) (example: We shouldn't try to guarantee that every group has the same quality of life).

Ideological mediators. Political ideology was calculated as the average score of responses to three items asking participants to indicate their political position generally, and on social and economic issues. Responses were recorded on a 7-point scale ranging from very liberal (1) to very conservative (7). Religiosity was measured using the brief version of the Santa Clara Strength of Religious Faith Questionnaire (Plante, Vallaeys, Sherman, \& Wallston, 2002) (example: My faith impacts many of my decisions). Free market ideology was measured using Heath and Gifford's 5-item scale (example: The free market system is likely to promote unsustainable consumption - reverse coded). ${ }^{17}$ Conspiracy mentality was measured using Bruder, Haffke, Neave, Nouripanah, and Imhoff's (2013) 5-item Conspiracy Mentality Questionnaire (example: I think that there are secret organizations that greatly influence political decisions).

Science-specific mediators. Science literacy was captured using an adapted version of the National Science Foundation's science literacy scale, a widely used true/false test of general science knowledge (example: Electrons are smaller than atoms; Miller, 2004). Following the recommendations of Roos (2014), I removed items relating to evolution, the big bang and continental drift from the original scale, as these tap a religious dimension. In addition to providing a true/false response for the remaining eight items, participants also reported how confident they were of each of their answers on a 4-point scale ranging from (Very unsure to Very sure). Answers and certainty were combined such that scores for each item ranged from 1 (incorrect and very sure) to 8 (correct and very sure). This approach is

\footnotetext{
${ }^{17}$ I must acknowledge the potential overlap between the conservatism and free market scales - particularly the conservatism item referring to views on economic issues (correlated with the free market scale $r=.49$ ). A principal components analysis (varimax rotation) of items from both scales indicated that the conservatism and free market items loaded differentially onto separate factors. I also repeated analyses with the 'economic issues' item removed from the conservatism scale and found that this did not substantially alter results.
} 
similar to Nisbet, Cooper, and Ellithrope (2015) and others in creating a continuous rather than dichotomous response set for these items. ${ }^{18}$ A scale formed from the average of these scores displayed only marginal reliability $(\alpha=.63)$ but was retained to allow comparison with other studies, given the widespread use of these items as a measure of scientific knowledge (Allum et al., 2008). Perceived credibility of scientists was measured using Hartman et al's (2017) 6-item Credibility of Science Scale (example: People trust scientists a lot more than they should). Following the recommendation of Hartman et al., scores on the COSS were reversed such that higher values indicated greater perceived credibility of science and scientists.

Rejection of science. Participant's beliefs about scientific issues were measured using five separate scales. Belief in the existence of human-caused climate change was measured using Lewandowsky et al.'s (2013) 5-item scale (example: Human CO2 emissions cause climate change). Dixons'(2016) 3-item scale measured agreement that GM food is safe to eat (GM food is safe to eat). Agreement that vaccines are safe and effective was measured with Lewandowsky et al.'s (2013) 5-item scale (Vaccines are a safe and reliable way to avert the spread of preventable diseases). Acceptance of the theory of evolution was measured with two items (Human beings, as we know them today, evolved from earlier species, and Humans evolved from earlier primate ancestors over millions of years; $r=.93)$. As there were no established and well-validated scales for assessing attitudes towards fluoridation, agreement that community water fluoridation is safe and effective was captured using a new scale with four items covering both the purported negative and positive health effects of fluoride (Royal Society of New Zealand, 2014), with an equal number of positive and reverse items (examples: Community water fluoridation is effective in reducing tooth decay; Adding even small amounts of fluoride to drinking water can have harmful effects on those who drink it). A principal components analysis (varimax rotation) indicated all items loaded (.84 or higher) onto a single factor explaining $81.54 \%$ of variance.

\section{Procedure}

Participants who registered to undertake the survey were directed to the Qualtrics survey platform (qualtrics.com) and provided informed consent before starting the survey and completing all measures. Participants read a debrief at the end of the survey and received

\footnotetext{
${ }^{18}$ Repeating analyses using a summed score of correct answers (range $0-8, M=6.72, S D=1.37$ ) produced substantially similar results.
} 
US\$1.60 compensation via the Academic Prolific platform. The study was approved by the Victoria University of Wellington School of Psychology Human Ethics Committee (application number: 0000025633). Data were cleaned, and descriptive and inferential statistics calculated in SPSS v23. A small number of missing values (.16\%) were imputed using the Expectation-Maximization procedure in SPSS.

\subsubsection{Results}

Descriptive statistics and correlations for Study 5 variables are reported in Table 6.3. Overall, the sample generally accepted the scientific consensus position across all five issues, with mean scores above the midpoint of the 1-7 range of each scale $\left(M_{\text {Climate }}=5.51, S D=\right.$ $1.30 ; M_{G M}=4.61, S D=1.62 ; M_{\text {Vaccination }}=6.00, S D=1.20 ; M_{\text {Fluoridation }}=4.83, S D=1.49$; $\left.M_{\text {Evolution }}=5.85, S D=1.56\right)$. Scores on the RWA $(M=3.06, S D=1.16)$ and $\operatorname{SDO}(M=2.52$, $S D=1.19$ ) scales were, on average, below the midpoint, as were scores on the free market ideology scale $(M=3.30, S D=1.20)$. The mean score on the political ideology scale was below the mid-point $(M=3.05, S D=1.59)$, indicating participants were, on average, slightly more liberal than conservative. The mean conspiracy mentality score $(M=4.74, S D=1.22)$ was above the midpoint, indicating that participants, overall, tended to agree more than disagree with the generic conspiracy statements in the scale.

Considering the correlations between variables, a consistent pattern emerges with RWA, SDO, religiosity, conservatism, free market ideology and conspiracy mentality all positively correlated with each other ( $r$ s .13 to .66). Scores on these scales all correlate negatively with perceived credibility of scientists, science literacy and all five of the rejection of science dependent variables ( $r$ s -.11 to -.58). Perceived credibility of scientists and science literacy were positively correlated with each other and the rejection of science variables ( $r \mathrm{~s}$ .23 to .59). All five of the rejection of science variables were positively correlated with each other ( $r$ s .26 to .57$)$. All correlations were significant at the $p<.001$ level. 
Table 6.3

Study 5 descriptive statistics and correlations

\begin{tabular}{|c|c|c|c|c|c|c|c|c|c|c|c|c|c|c|c|c|c|}
\hline & $M$ & $S D$ & $\alpha$ & 1 & 2 & 3 & 4 & 5 & 6 & 7 & 8 & 9 & 10 & 11 & 12 & 13 & 14 \\
\hline 1. Age & $25-29^{a}$ & - & - & - & & & & & & & & & & & & & \\
\hline 2. $\quad$ Gender $(M=1)^{b}$ & $49.3 \%$ & - & - & -.14 & - & & & & & & & & & & & & \\
\hline 3. Climate change & 5.51 & 1.30 & .88 & -.19 & -.01 & - & & & & & & & & & & & \\
\hline 4. Genetic modification & 4.61 & 1.62 & .92 & -.17 & .16 & .29 & - & & & & & & & & & & \\
\hline 5. Vaccination & 6.00 & 1.20 & .89 & -.19 & .00 & .45 & .55 & - & & & & & & & & & \\
\hline 6. Fluoridation & 4.83 & 1.49 & .92 & -.03 & .10 & .35 & .54 & .57 & - & & & & & & & & \\
\hline 7. Evolution & 5.85 & 1.56 & .96 & -.16 & .06 & .49 & .39 & .45 & .34 & - & & & & & & & \\
\hline 8. RWA & 3.06 & 1.16 & .93 & .21 & .04 & -.56 & -.37 & -.42 & -.26 & -.49 & - & & & & & & \\
\hline 9. $\mathrm{SDO}$ & 2.52 & 1.19 & .89 & .08 & .21 & -.51 & -.18 & -.31 & -.20 & -.24 & .58 & - & & & & & \\
\hline 10. Conservatism & 3.05 & 1.59 & .92 & .12 & .21 & -.53 & -.23 & -.32 & -.22 & -.38 & .66 & .57 & - & & & & \\
\hline 11. Religiosity & 2.67 & 1.95 & .95 & .23 & -.10 &.- .33 & -.34 & -.29 & -.17 & -.51 & .50 & .18 & .34 & - & & & \\
\hline 12. Free market ideology & 3.30 & 1.20 & .82 & .10 & .06 & -.47 & -.21 & -.28 & -.22 & -.32 & .51 & .44 & .49 & .18 & - & & \\
\hline 13. Conspiracy mentality & 4.74 & 1.22 & .82 & -.06 & -.03 & -.23 & -.36 & -.28 & -.34 & -.19 & .21 & .16 & .13 & .16 & .15 & - & \\
\hline 14. Scientists' credibility & 5.05 & 1.49 & .92 & -.15 & -.04 & .59 & .45 & .58 & .37 & .53 & -.58 & -.45 & -.46 & -.51 & -.37 & -.31 & - \\
\hline 15. Science literacy & 6.72 & 1.06 & .63 & .02 & .11 & .23 & .34 & .34 & .24 & .24 & -.28 & -.14 & -.11 & -.13 & -.21 & -.25 & .32 \\
\hline
\end{tabular}

Note. The variables 'Climate', 'Genetic modifaction', 'Vaccination', 'Fluoridation', and 'Evolution' reflect (respectively) agreement that: human activities cause climate change; GM foods are safe to eat; vaccines are safe and effective; water fluoridation is safe and effective; and humans evolved from earlier species. 'Scientists' credibility' refers to perceieved credibility of scientists.

${ }^{a}$ Median age bracket. ${ }^{b}$ Excludes non-binary gender $(N=641)$.

Correlation coefficients in bold are significant at the $p<.001$ level 


\section{Ideological attitudes and rejection of science}

To examine the unique effects of ideological attitudes, each rejection of science variable was regressed onto RWA and SDO with gender and age as covariates (as in Study 4). All regressions were significant $(F \mathrm{~s}(4,363)>15.89, p \mathrm{~s}<.001)$. The results, shown in Table 6.4, reveal RWA is a consistent predictor of rejection of science across all five issues while SDO predicts rejection of climate change, vaccination and fluoridation. Thus, we find that individuals who endorse authoritarian and socially conforming views are more likely to disagree with the established scientific consensus position on debated scientific issues. Individuals who are more accepting of social inequality are also more likely to reject the scientific consensus on climate change, vaccination and fluoridation, but not GM food or evolution.

Table 6.4

Demographic and ideological predictors of acceptance of science (US sample)

\begin{tabular}{|c|c|c|c|c|c|}
\hline & $\begin{array}{l}\text { Climate } \\
\text { change }\end{array}$ & $\begin{array}{l}\text { Genetic } \\
\text { modification }\end{array}$ & Vaccination & Fluoridation & Evolution \\
\hline Age & $-.08 * * *$ & $-.07 * *$ & $-.11 * *$ & .04 & -.05 \\
\hline Gender $(M=1)$ & .05 & $.16^{* * *}$ & .02 & $.14^{* * * *}$ & .06 \\
\hline RWA & $-.38 * * *$ & $-.36 * * *$ & $-.32 * * *$ & $-.22 * * *$ & $-.51 * * *$ \\
\hline SDO & $-.29 * * *$ & -.01 & $-.12 * *$ & $-.10^{*}$ & .04 \\
\hline Adjusted $\mathrm{R}^{2}$ & $.37 * * *$ & $.17 * * *$ & $.19 * * *$ & $.09 * * *$ & $.24 * * *$ \\
\hline
\end{tabular}

In agreement with Study 4, these models show that high RWA and SDO individuals are more likely to reject science but offer little insight into the reasons why. To further examine the relationships between ideological attitudes and rejection of science I created a model incorporating ideological and science-specific latent constructs as mediators of the effect of ideological attitudes on the five rejection of science outcomes.

\section{Mediators of the relationship between ideological attitudes and rejection of science}

A structural equation model was constructed using the R package lavaan (Rosseel, 2012). Stable ideological attitudes RWA and SDO were considered exogenous variables 
predicting rejection of science on the five separate issues. Political conservatism, religiosity, free market ideology, conspiracy mentality, science literacy and perceived credibility of scientists were included in the model as potential mediators of these effects. That is, each rejection of science latent variable was regressed onto ideological attitudes as well as ideological and science-specific mediators. Ideological and science-specific mediators were also regressed onto ideological attitudes. I note that there may be theoretical justifications for including science-specific variables as a second set of mediators between ideological mediators and rejection of science as these could be assumed to be more psychologically proximal to beliefs about scientific issues (c.f. Rutjens et al., 2017). However, in pursuit of parsimony in the current model, I include all mediators in parallel. My primary focus here is to explain the association between RWA/SDO and rejection of science. Items for the RWA, SDO and science literacy latent variables were randomly parcelled to create three observed indicators for each variable (Little, Cunningham, Shahar, \& Widaman, 2009). All mediator variables were correlated with each other. In the absence for theoretical grounds for omission from the starting model, all paths were retained in the final model although only significant paths are reported here. Given the significant influence of demographic controls (see Table 6.4), age and gender were included as covariates (effects are not reported here but can be found in Appendix G). I did not remove non-significant paths or make adjustments based on modification indices. Standard errors were bootstrapped (5000 samples) and an MLM estimator was used to calculate fit statistics robust to non-normality.

The resulting model (Figure 6.1) displayed reasonable fit $\left(\chi^{2}(1278)=3234.04, \mathrm{CFI}=\right.$ $.912, \mathrm{RMSEA}=.049[.047, .051], \mathrm{SRMR}=.063)$, according to the criteria recommended by Hu and Bentler (1999): RMSEA <.06 and SRMR <.08 (although the CFI was below the suggested .95 cut-off). 


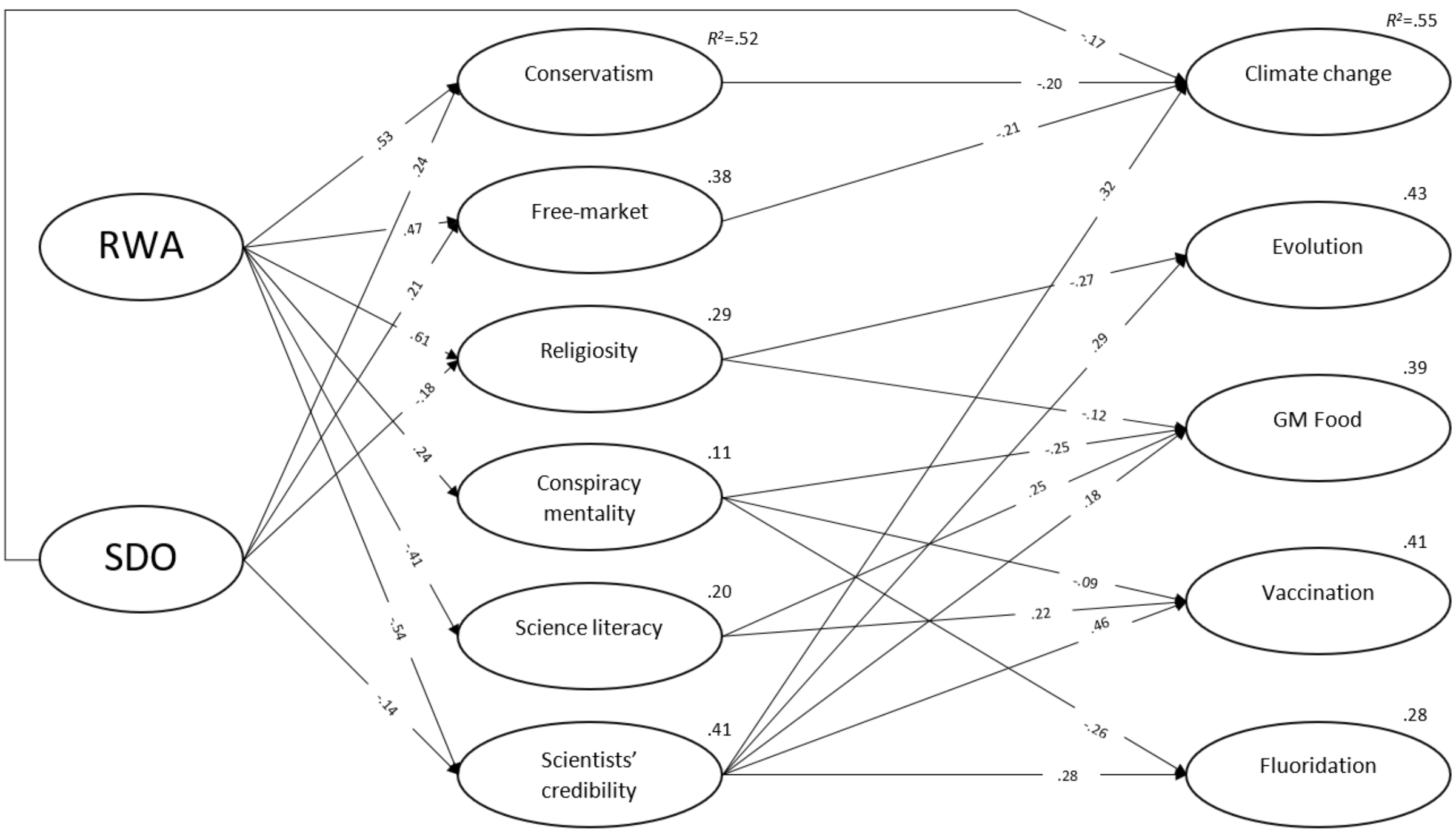

Figure 6.1. Mediators of the effects of RWA and SDO on acceptance of science (Study 5). Age and gender covariates, covariances, and nonsignificant paths $(\mathrm{p}>.05)$ not shown (estimates for all parameters are reported in Appendix G) 
I will first consider the effects of ideological attitudes on the mediating variables. RWA is a significant predictor of all mediators in the model, positively associated with conservatism, religiosity, free market ideology and conspiracy mentality, and negatively associated with science literacy and perceived credibility of scientists. SDO also predicts conservatism and free market ideology but in contrast to RWA is a negative predictor of religiosity. SDO also negatively predicts perceived credibility of scientists but has no relationship with science literacy or conspiracy mentality.

Turning to the rejection of science variables, we find that RWA has no direct effect on any of the latent variables representing agreement with the scientific consensus. This indicates that any effects of RWA on these variables are mediated by the other ideological and science-specific variables in the model. There is a single direct (negative) effect of SDO on climate change belief and all other effects of SDO on rejection of science variables are mediated. Estimates of the total effects (combined indirect and direct effects) of ideological attitudes on rejection of science are shown in Table 6.5. The pattern of results aligns with the regression analyses reported in Table 6.4, with exception of the association between SDO and fluoride, which was significant in the OLS model but failed to reach significance in the current SEM model.

Table 6.5

Total effects of RWA and SDO on acceptance of science latent variables (Study 5)

\begin{tabular}{|c|c|c|c|c|}
\hline Ideological attitude & Dependent variable & $\beta$ & $b$ & $95 \mathrm{CI}$ \\
\hline \multirow[t]{5}{*}{ RWA } & Climate change & -.39 & -.50 & {$[-.64,-.36]$} \\
\hline & GM food & -.39 & -.52 & {$[-.67,-.36]$} \\
\hline & Vaccination & -.31 & -.34 & {$[-.47,-.19]$} \\
\hline & Fluoridation & -.24 & -.36 & {$[-.55,-.17]$} \\
\hline & Evolution & -.55 & -.79 & {$[-.97,-.62]$} \\
\hline \multirow[t]{5}{*}{ SDO } & Climate change & -.32 & -.37 & {$[-.51,-.24]$} \\
\hline & GM food & .02 & .02 & {$[-.12, .18]$} \\
\hline & Vaccination & -.13 & -.13 & {$[-.26,-.01]$} \\
\hline & Fluoridation & -.10 & -.13 & {$[-.32, .05]$} \\
\hline & Evolution & .07 & .10 & {$[-.07, .26]$} \\
\hline
\end{tabular}

Standardised $(\beta)$ and unstandardised coefficients $(b)$ with bootstrapped (bias-corrected; 5,000 samples) confidence intervals shown. Bold values denote significant effects (bootstrapped confidence interval does not include zero). 
Due to space I will not report the large number of individual indirect effects in the model here (see Table G.2, Appendix G). To summarise, any two connecting significant paths (ideological attitude $\rightarrow$ mediator $\rightarrow$ rejection of science) represent a significant indirect effect (i.e. the bootstrapped $95 \%$ confidence interval did not include zero). For example, the total effect of RWA on GM food was mediated by religiosity $(\beta=-.07, b=-.10$, bootstrapped 95CI $[-.19,-.02])$, conspiracy mentality $(\beta=-.06, b=-.08,[-.14,-.03])$, science literacy $(\beta=-$ $.10, b=-.14,[-.23,-.07])$ and perceived credibility of scientists $(\beta=-.10, b=-.13,[-.23,-$ .03]). Of note was the effect of SDO on evolution and GM food beliefs. While the total effect of SDO on these variables was not significant (see Table 6.5), examination of indirect effects revealed significant opposing effects, with a positive effect mediated via religiosity ( $\beta_{\text {evolution }}$ $\left.=.05, b=.06,[.02, .13] ; \beta_{G M}=.02, b=.03,[.005, .07]\right)$ and negative indirect effect via credibility of scientists $\left(\beta_{\text {evolution }}=.04, b=.05,[-.12,-.01] ; \beta_{G M}=-.02, b=-.03,[-.08,-.005]\right)$. In the case of evolution, free market ideology also mediates a negative effect of SDO $(\beta=-$ $.02, b=-.03,[-.07,-.004])$ due to a borderline significant effect of free market ideology on evolution beliefs $(\beta=-.11, p=.06$; not shown in figure). SDO had a significant negative indirect effect of SDO on fluoridation via credibility of scientists $(\beta=-.04, b=-.05,[-.12,-$ $.01]$ ), though this did not result in a significant total effect. Thus, SDO does have indirect and at times conflicting influences on GM food, fluoride and evolution beliefs. These results highlight the importance of exploring mediation effects even in the absence of a significant total effect (Hayes, 2009).

Overall, the analysis reveals that RWA is a significant predictor of rejection of science across domains, but the combination of variables which mediate this effect changes depending on the scientific issue under consideration. Perceived credibility of scientists, however, emerges as a consistent mediator in this context. While SDO only demonstrated a significant total effect on two rejection of science variables (climate change and vaccination), SDO also had a consistent negative indirect effect on all issues mediated via perceived credibility of scientists, and in the case of evolution and GM food, a significant positive indirect effect via religiosity.

Considering the total variance explained, I report that over half of the variance in climate change beliefs $\left(R^{2}=.55\right)$ and around $40 \%$ of the variance in GM food, vaccination and evolution beliefs $\left(R^{2} \mathrm{~s} .39, .41\right.$, and .43 , respectively) is explained by predictors in the model. The model explained a lower, but still significant amount of variance in the fluoridation beliefs $\left(R^{2}=.28\right)$. 


\section{Modelling overall science acceptance}

The main model presented in Figure 6.1 examines beliefs about five separate scientific issues where the scientific consensus is challenged in public discourse. However there are other scientific issues where segments of the population reject the best available evidence (e.g. HIV denialism, 'flat Earth' beliefs), and it is almost certain that scientific and technological developments will give rise to new battlegrounds for science and public opinion. To identify predictors of a general propensity to accept or reject science, I modelled a second order science acceptance latent variable based on the five rejection of science variables. ${ }^{19}$

As a first step, I conducted a principal components analysis using varimax rotation to determine the factor structure of the five rejection of science scales (the mean scores of each scale). Examination of scree plot and Eigenvalues indicated a single factor solution $($ Eigenvalue $=2.79$, explaining $55.74 \%$ of variance $)$, with all scales loading $>.68$.

Given the single factor structure of the rejection of science scales, I proceeded to model a second-order latent variable based on the five rejection of science latent variables, with the same set of exogenous predictors, mediating variables, and demographic covariates as the previous model. The resulting model displayed marginal fit $\left(\chi^{2}(1323)=3606.82, \mathrm{CFI}=.897\right.$, RMSEA $=.052[.050, .054]$, SRMR $=.08)$. Significant effects in the model are displayed in Figure 6.2, and all direct, indirect and total effects are reported in Table 6.6.

\footnotetext{
${ }^{19}$ Examples of this approach (using single items rather than latent variables) can be found in Lewandowsky et al. (2012) and Lewandowsky, Oberauer, et al. (2013).
} 


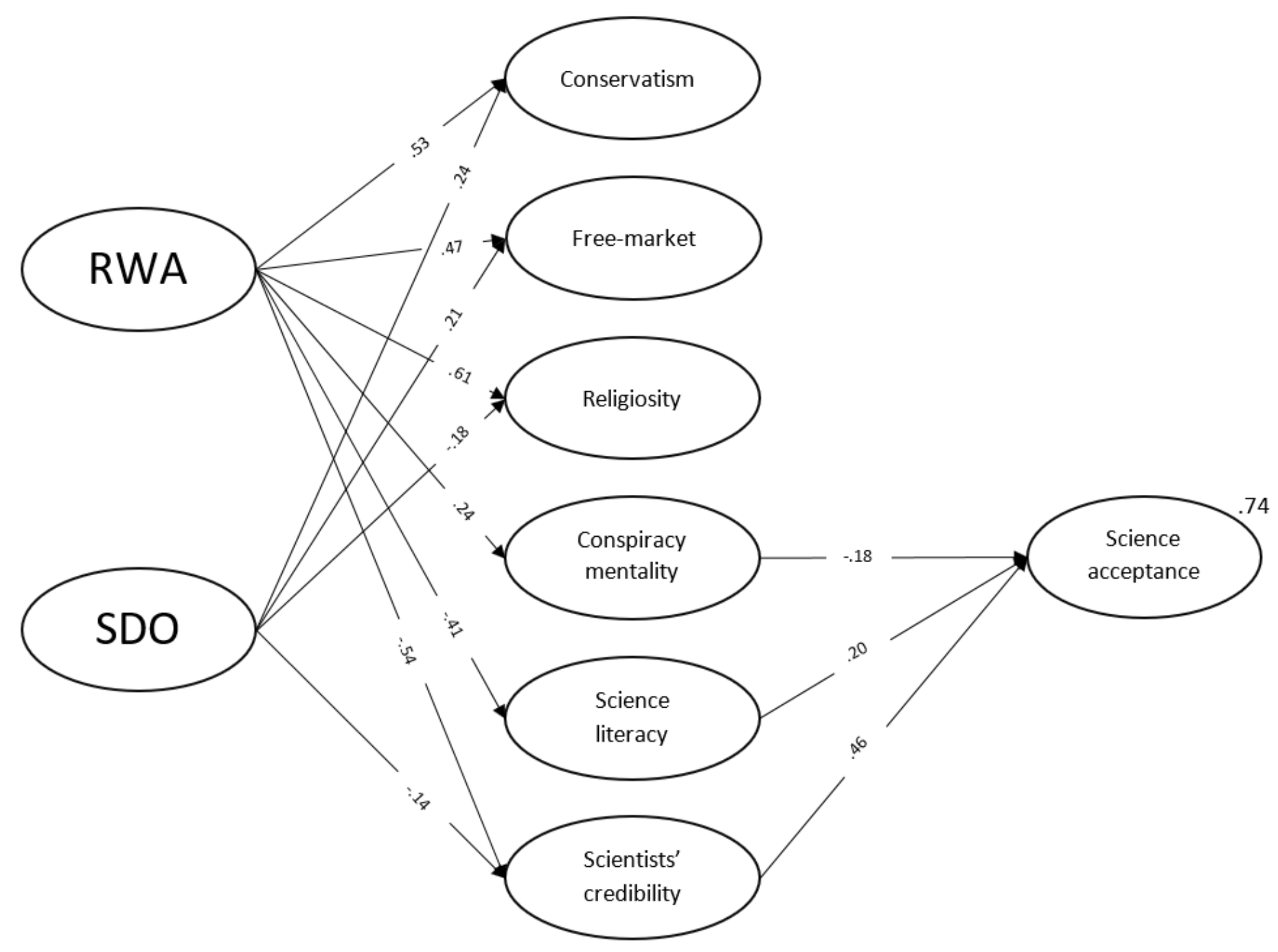

Figure 6.2. Mediators of the effects of RWA and SDO on a second order latent science acceptance variable (Study 5). Non-significant paths, covariences, age and gender covariates, not shown (estimates for all parameters are reported in Appendix G). 
Considering the total effects of ideological attitudes on the science acceptance variable (Table 6.6), we find that RWA has a significant total effect on science acceptance mediated by conservatism, free market ideology, conspiracy mentality, perceived credibility of scientists and science literacy. SDO also has a significant total effect on science acceptance, mediated via conservatism, free market beliefs and credibility. The significant indirect effects via conservatism and free market beliefs are due to these mediators having borderline significant effects on science acceptance $\left(\beta_{\text {Conservatism }}=-.11, p=.05, \beta_{\text {Free market }}=-.10, p=.07\right.$; not shown in model). The model explained almost three-quarters of the variation in the second order science acceptance latent variable $\left(R^{2}=.74\right)$, indicating that perceived credibility of scientists, conspiracy mentality and science literacy in combination powerfully predict one's overall tendency to endorse the mainstream scientific consensus. The model also indicates that RWA and, to a lesser extent, SDO underpin these ideological and sciencespecific predictors. However, as can be seen in Figure 6.1, each scientific issue also has its own unique combination of predictors.

Table 6.6

Indirect, direct and total effects of RWA and SDO on overall science acceptance (Study 5)

\begin{tabular}{|c|c|c|c|c|}
\hline Independent variable & Mediator & $\beta$ & $b$ & $95 \mathrm{CI}$ \\
\hline \multirow[t]{8}{*}{ RWA } & Religiosity & -.04 & -.03 & {$[-.08, .03]$} \\
\hline & Conservatism & -.06 & -.05 & {$[-.11,-.004]$} \\
\hline & Free market ideology & -.05 & -.04 & {$[-.09,-.001]$} \\
\hline & Conspiracy mentality & -.04 & -.04 & {$[-.07,-.02]$} \\
\hline & Scientists' credibility & -.25 & -.22 & {$[-.30,-.15]$} \\
\hline & Science literacy & -.08 & -.07 & {$[-.13,-.03]$} \\
\hline & Direct effect & -.03 & -.03 & {$[-.16, .13]$} \\
\hline & Total effect & -.55 & -.48 & {$[-.61,-.35]$} \\
\hline \multirow[t]{8}{*}{ SDO } & Religiosity & .01 & .01 & {$[-.004, .03]$} \\
\hline & Conservatism & -.03 & -.02 & {$[-.05,-.002]$} \\
\hline & Free market ideology & -.02 & -.02 & {$[-.04,-.002]$} \\
\hline & Conspiracy mentality & -.02 & -.01 & {$[-.04, .003]$} \\
\hline & Scientists' credibility & -.06 & -.05 & {$[-.11,-.01]$} \\
\hline & Science literacy & .01 & .00 & {$[-.02, .03]$} \\
\hline & Direct effect & -.03 & -.02 & {$[-.13, .08]$} \\
\hline & Total effect & -.14 & -.11 & {$[-.23,-.004]$} \\
\hline
\end{tabular}

Standardised $(\beta)$ and unstandardised coefficients $(b)$ with bootstrapped (5,000 samples) confidence intervals shown. Bold values denote significant effects (bootstrapped confidence interval does not include zero). 


\subsubsection{Bridging comment}

The results of Study 5 indicate that US adults who are more authoritarian are more likely to reject science. High SDO individuals are also more likely to reject science on some issues, but not others. The findings broadly match the results of Study 4 (NZ students) although there was no relationship between SDO and rejection of GM food science in the US sample.

Study 5 also highlighted the mediating role of several ideological and science-specific factors. The relative importance of these mediators depended on the scientific domain in question. The only consistent mediator across all issues was the perceived credibility of scientists (analogous to trust in scientists). A broader discussion of these findings and comparisons between studies will follow the final study, Study 6 .

\subsection{STUDY 6: INVESTIGATING THE RELATIONSHIP BETWEEN IDEOLOGICAL ATTITUDES AND REJECTION OF SCIENCE IN A LARGE NZ PUBLIC SAMPLE}

\subsubsection{Introduction}

Study 5 identified the mediators of the relationship between ideological attitudes and rejection of science in a US online sample sourced through a participant recruitment platform. Do these finding generalise to a larger, more diverse public sample in a different national context? Study 6 seeks to replicate the findings of Study 5 using a large non-representative New Zealand sample.

\subsubsection{Methods}

\section{Participants}

Participants were recruited through a national newspaper (see Procedure below). Only participants who completed the survey were included in analyses. A total of 9,126 New Zealand-based individuals completed the survey. Participants who failed either of two attention checks (instructed-response items, e.g. 'Please select "agree" for this statement'; Gummer et al., 2018) embedded in the survey were removed from the sample (1025 participants). ${ }^{20}$

The final sample displayed a relatively even split of gender with $46.8 \%$ of participants identifying as women, $53.2 \%$ as men and $0.08 \%$ reporting non-binary or no gender (due to

\footnotetext{
${ }^{20}$ This is within the range reported in previous research (Hauser \& Schwarz, 2016)
} 
low numbers these individuals were removed from analyses including gender as a covariate). The median age bracket was 45-49 years.

In terms of reported ethnicity, the sample was primarily European/ Pākehā (91.5\%), followed by Māori (6.4\%), Asian (1.8\%) and Pacific Peoples (1.1\%). A substantial number of participants $(7.1 \%)$ provided an 'Other' response. Ethnicity response options were not mutually exclusive; $7.2 \%$ of participants selected more than one option. Reported highest qualification was as follows: high school completion or lower, $17.3 \%$; partially completed bachelor's degree/trade certificate, $12.8 \%$, completed bachelor's degree/trade certificate, $42.2 \%$ (median and modal response); and postgraduate degree, $27.7 \%$. Demographically, the sample was older, more educated and skewed towards European ethnicity compared to the general New Zealand public (Statistics New Zealand, 2013)

\section{Materials}

Measures used in the survey were identical to those in Study 5 (scale $\alpha$ s are reported in Table 6.7). The survey was undertaken in collaboration with other researchers and included a range of other items unrelated to the current study.

\section{Procedure}

Participants were recruited through a collaboration with a large New Zealand weekend newspaper. This approach has proven successful in gathering relatively large and broadly representative samples previously (e.g. M. S. Wilson, Bulbulia, \& Sibley, 2014). Several news articles highlighting the survey were published in print and online, providing participants with a link to complete the survey on the Qualtrics platform. The survey was presented as a study of New Zealanders opinions about scientific, health and environmental issues.

Participants who opted to take part in the study accessed the survey via a link provided in both print and online versions of newspaper articles highlighting the survey. The link directed participants to the Qualtrics survey platform where they provided informed consent and completed the survey. Upon completion participants were offered the option to enter a prize draw to win an incentive prize (an Apple iPad Pro). The study was approved by the Victoria University of Wellington School of Psychology Human Ethics Committee (application number: 0000025633). Data were cleaned, and descriptive and inferential statistics calculated in SPSS v23. A small number of missing values $(0.52 \%)$ were imputed using the Expectation-Maximization procedure in SPSS. 


\subsubsection{Results}

Descriptive statistics and bivariate correlations for the variables included in the study are shown in Table 6.7. We find a pattern of correlations essentially identical to Study 5. RWA, SDO, religiosity, conservatism, free market ideology and conspiracy mentality all positively correlated with each other ( $r$ s .04 to .65). I must note that the correlations between religiosity and SDO $(r=.04, p<.001)$ and religiosity and free market ideology $(r=.05, p<$ .001) while significant, are relatively small. With one exception, scores on these scales all correlate negatively with perceived credibility of scientists, science literacy and all five of the rejection of science dependent variables ( $r$ s -.18 to -.57). The exception was the relationship between religiosity and science literacy which was nonsignificant (in contrast to the results of Study 5). Perceived credibility of scientists and science literacy were positively correlated with each other and the rejection of science variables ( $r$ s .19 to .47). All five of the rejection of science variables were positively correlated with each other ( $r$ s .20 to .54). Barring the noted exception, all correlations were significant at the $p>.001$ level. 
Table 6.7

Study 6 descriptive statistics and correlations

\begin{tabular}{|c|c|c|c|c|c|c|c|c|c|c|c|c|c|c|c|c|c|c|}
\hline & $M$ & $S D$ & $\alpha$ & 1 & 2 & 3 & 4 & 5 & 6 & 7 & 8 & 9 & 10 & 11 & 12 & 13 & 14 & 15 \\
\hline 1. Age & $45-49^{\mathrm{a}}$ & - & - & 一 & & & & & & & & & & & & & & \\
\hline 2. Gender $(\mathrm{M}=1)^{\mathrm{a}}$ & $53.2 \%$ & - & - & .11 & - & & & & & & & & & & & & & \\
\hline 3. Climate change & 5.56 & 1.21 & .86 & -.10 & .02 & - & & & & & & & & & & & & \\
\hline 4. Genetic modification & 4.84 & 1.38 & .90 & -.03 & .17 & .20 & - & & & & & & & & & & & \\
\hline 5. Vaccination & 6.35 & 0.87 & .71 & -.03 & .04 & .29 & .40 & 一 & & & & & & & & & & \\
\hline 6. Fluoridation & 5.80 & 1.20 & .93 & .07 & .13 & .28 & .51 & .54 & 一 & & & & & & & & & \\
\hline 7. Evolution & 6.16 & 1.39 & .94 & .00 & .10 & .34 & .28 & .28 & .29 & 一 & & & & & & & & \\
\hline 8. RWA & 3.18 & 0.90 & .90 & .06 & .00 & -.48 & -.24 & -.20 & -.23 & -.41 & - & & & & & & & \\
\hline 9. SDO & 2.57 & 1.03 & .85 & .06 & .19 & -.45 & -.09 & -.18 & -.15 & -.16 & .50 & 一 & & & & & & \\
\hline 10. Conservatism & 3.32 & 1.24 & .86 & .09 & .08 & -.47 & -.14 & -.14 & -.14 & -.31 & .65 & .52 & 一 & & & & & \\
\hline 11. Religiosity & 2.15 & 1.60 & .93 & .06 & -.08 & -.18 & -.17 & -.13 & -.12 & -.58 & .32 & .04 & .22 & 一 & & & & \\
\hline 12. Free market ideology & 2.97 & 1.15 & .87 & -.06 & -.03 & -.47 & -.07 & -.14 & -.16 & -.17 & .46 & .43 & .46 & .05 & 一 & & & \\
\hline 13. Conspiracy mentality & 3.78 & 1.16 & .80 & -.05 & -.10 & -.31 & -.41 & -.32 & -.42 & -.24 & .34 & .23 & .23 & .12 & .18 & - & & \\
\hline 14. Scientist's credibility & 5.21 & 1.14 & .87 & -.02 & .06 & .47 & .40 & .40 & .44 & .41 & -.38 & -.30 & -.31 & -.26 & -.26 & -.46 & - & \\
\hline 15. Science literacy & 7.04 & 0.88 & .57 & .06 & .20 & .19 & .29 & .21 & .26 & .19 & -.22 & -.09 & -.11 & -.02 & -.20 & -.26 & .26 & \\
\hline
\end{tabular}

Note. The variables 'Climate', 'Genetic modifaction', 'Vaccination', 'Fluoridation', and 'Evolution' reflect (respectively) agreement that: human activities cause climate change; GM foods are safe to eat; vaccines are safe and effective; water fluoridation is safe and effective; and humans evolved from earlier species. 'Scientists' credibility' refers to perceieved credibility of scientists.

${ }^{\mathrm{a}}$ Median age bracket. ${ }^{\mathrm{b}}$ Excludes non-binary gender $(N=8038)$.

Correlation coefficients in bold are significant at the $p<.001$ level. 


\section{Ideological attitudes and rejection of science}

As in Study 5, as a first step, I regressed rejection of science variables onto age, gender, RWA and SDO. The results, shown in Table 6.8, largely mirror those of Study 5: RWA is a significant predictor of rejection of science across all issues and SDO predicts rejection of science regarding climate change, vaccination and fluoridation but not GM food. In contrast to Study 5, I also report a small but significant positive effect of SDO on belief in evolution.

Table 6.8

Demographic and ideological predictors of acceptance of science (NZ sample)

\begin{tabular}{llllll}
\hline & $\begin{array}{c}\text { Climate } \\
\text { change }\end{array}$ & GM Food & Vaccination & Fluoridation & Evolution \\
\hline Age & $-.07 * * *$ & $-.03 * *$ & $-.02 *$ & $.07 * * *$ & .01 \\
Gender $(\mathrm{M}=1)$ & $.08 * * *$ & $.17 * * *$ & $.06 * * *$ & $.13 * * *$ & $.09 * * *$ \\
RWA & $-.33 * * *$ & $-.23 * * *$ & $-.14 * * *$ & $-.19 * * *$ & $-.43 * * *$ \\
SDO & $-.29 * * *$ & -.00 & $-.12 * * *$ & $-.09 * * *$ & $.04 * * *$ \\
Adjusted $\mathrm{R}^{2}$ & $.30 * * *$ & $.09 * * *$ & $.05 * * *$ & $.08 * * *$ & $.18 * * *$ \\
\hline
\end{tabular}
${ }^{*} p<.05,{ }^{* *} p<.01,{ }^{* * *} p<.001$.

\section{Mediators of the relationship between ideological attitudes and rejection of science}

Following the same procedure as Study 5, I constructed a structural equation model to examine the ideological and science-specific mediators of the relationship between ideological attitudes and rejection of science. The resulting model displayed adequate fit $(\chi 2(1278)=16454.37, \mathrm{CFI}=.935, \mathrm{RMSEA}=.041[.040, .041], \mathrm{SRMR}=.040)$. The associations between latent variables are shown in Figure 6.3. To facilitate interpretation of the results and comparison with Study 5, Figure 6.3 only displays significant paths with an absolute co-efficient value greater than $.10 .^{21}$ All parameter estimates are shown in Appendix $\mathrm{H}$.

\footnotetext{
${ }^{21}$ Due to the large sample size there were many parameters which were statistically significant but of questionable practical significance (Kirk, 1996). I decided to use the criterion of $<|.10|$ maintain focus on larger effect sizes. I selected this value after examination of the Study 5 model, where nearly all significant paths were greater than $|\cdot 10|$.
} 


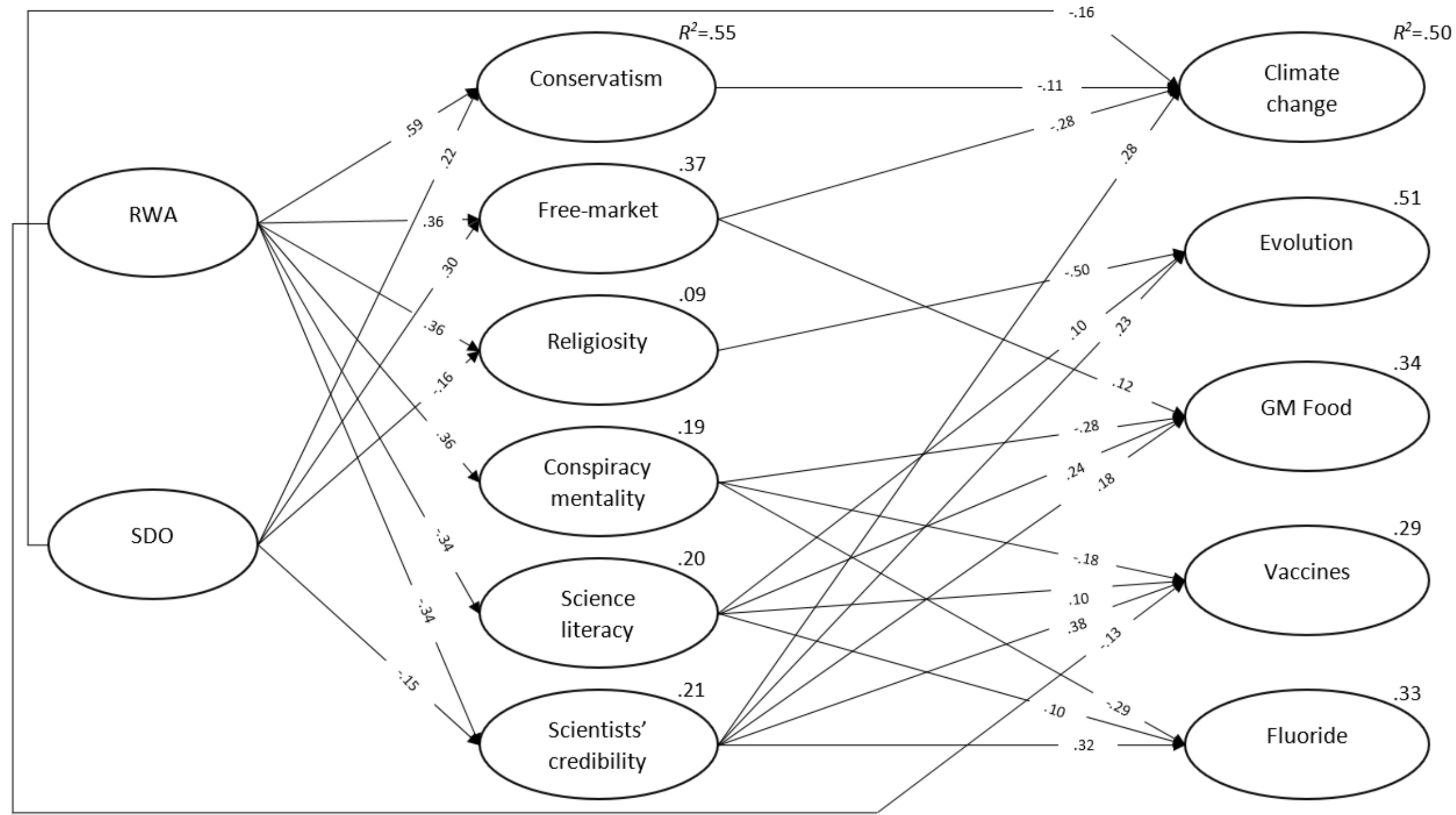

Figure 6.3. Mediators of the effects of RWA and SDO on acceptance of science (Study 6). Age and gender covariates, coveriances, and nonsignificant paths and paths with a coefficient $<|.10|$ are not shown (estimates for all parameters are reported in Appendix $\mathrm{H}$ ). 
The results largely follow the pattern of effects seen in Study 5. The RWA latent variable predicts political conservatism, free market ideology, religiosity and conspiracy mentality. RWA is also a negative predictor of science literacy and perceived credibility of scientists. In contrast to Study 5, there is also a direct negative effect of RWA on vaccine beliefs. This indicates that the relationship between RWA and anti-vaccination attitudes is not fully explained by the mediators included in the model. The pattern of significant effects of SDO in the model aligns with those reported in Study 5: SDO is a positive predictor of political conservatism and free market endorsement, and a negative predictor of religiosity and perceived credibility of scientists. Again, we also find that SDO has a significant negative direct effect on climate change beliefs.

Turning to the effects of the mediators on rejection of science latent variables, we find a similar pattern to that reported in Study 5. For brevity, I will focus on the few differences between the current model and the results of Study 5. In contrast to the Study 5 model, science literacy here was a significant predictor of the fluoride and evolution latent variables and free market ideology was a predictor of the GM food safety latent variable (these paths were not significant in Study 5). The effect of religiosity on GM food safety $(\beta=-.06, p<$ .001 ), while significant, failed to meet the $>|.10|$ criterion and thus is not shown in the model. This path was also significant in the Study 5 model.

The total effects of RWA and SDO on rejection of science variables (Table 6.9) are consistent with the results of the regression analyses shown in Table 6.8. RWA is a predictor of rejection of science across all domains examined, while SDO predicts rejection of climate, vaccine and fluoride science, and predicts acceptance of evolution science. 
Table 6.9

Total effects of RWA and SDO on acceptance of science latent variables (Study 6)

\begin{tabular}{|c|c|c|c|c|}
\hline Ideological attitude & Dependent variable & $\beta$ & $b$ & $95 \mathrm{CI}$ \\
\hline \multirow[t]{5}{*}{ RWA } & Climate change & -.35 & -.83 & {$[-.91,-.75]$} \\
\hline & GM food & -.26 & -.56 & {$[-.63,-.48]$} \\
\hline & Vaccination & -.10 & -.13 & {$[-.17,-.09]$} \\
\hline & Fluoridation & -.19 & -.40 & {$[-.47,-.33]$} \\
\hline & Evolution & -.42 & -.97 & {$[-1.05,-.90]$} \\
\hline \multirow[t]{5}{*}{ SDO } & Climate change & -.31 & -.45 & {$[-.49,-.40]$} \\
\hline & GM food & .02 & .02 & {$[-.02, .07]$} \\
\hline & Vaccination & -.15 & -.11 & {$[-.14,-.09]$} \\
\hline & Fluoridation & -.09 & -.11 & {$[-.15,-.06]$} \\
\hline & Evolution & .05 & .07 & {$[.03, .12]$} \\
\hline
\end{tabular}

Standardised $(\beta)$ and unstandardised coefficients $(b)$ with bootstrapped (bias corrected; 5,000 samples) confidence intervals shown. Bold values indicate a significant total effect (bootstrapped confidence interval does not include zero).

The direct, indirect and total effects of ideological attitudes on rejection of science are reported in Appendix G. All indirect effects represented by connecting paths in Figure 6.3 (ideological attitude $\rightarrow$ mediator $\rightarrow$ rejection of science) are significant. There are also several small indirect effects not shown in Figure 6.3 as one or more of the coefficients failed to reach the $>|.10|$ criterion. As with Study 5, we see considerable heterogeneity in which ideological and science-specific variables mediate the effects of RWA and SDO on rejection of science. The only consistent mediator is perceived credibility of scientists which predicts rejection of science across all five issues and is predicted by RWA and SDO.

In sum, using data from a large New Zealand public survey, we find similar results to the study undertaken with a US online sample: RWA predicts rejection of science across a range of domains as does SDO, with some exceptions. The mediators of these effects vary according to the scientific positions in question. Again, we find that the perceived credibility 
of scientists plays a consistent role in mediating the effects of both RWA and SDO on all rejection of science variables considered in the model. ${ }^{22}$

\section{Modelling overall science acceptance}

To examine the effects of ideological attitudes on a general propensity to accept or reject scientific findings I again constructed a model incorporating a second-order latent variable based on the five rejection of science latent variables. As in Study 5, I first undertook a principal components analysis using varimax rotation to determine the factor structure of the five rejection of science scales. Examination of scree plot and Eigenvalues indicated a single factor solution (Eigenvalue $=2.39$, explaining $47.89 \%$ of variance), with all scale scores loading $>.57$.

I constructed a SEM identical in specification to that created in Study 5. The resulting model displayed adequate fit $(\chi 2(1323)=22,483.21, \mathrm{CFI}=.909, \mathrm{RMSEA}=.047$ [.047, .048], SRMR = .073). The model is shown in Figure 6.4. Again, I limit displayed paths to those which have an absolute coefficient value greater than .10. The effects of RWA and SDO on the ideological and science-specific mediators are unchanged from the previous model. The model predicted a substantial amount of variation (72\%) in general science acceptance.

\footnotetext{
${ }^{22}$ I this section I have discussed differences between US and NZ samples in terms of significant effects in the models presented. Multigroup analysis represents more formal approach to comparing the results between Study 5 and Study 6. This involves combining the datasets, establishing metric and scalar invariance between groups and undertaking a more nuanced (and lengthy) series of analyses comparing the impact on model fit of constraining and releasing individual regression coefficients between samples, including tests of statistical significance. This analysis was undertaken (and metric and structural invariance established) but results are not reported due to concerns regarding the very unbalanced group sizes. Recently proposed subsampling methods accounting for this imbalance (Yoon \& Lai, 2018) were judged to be too computationally intensive for the model presented here (requiring more than 7,400 separate models to be fitted to compare each path between US and NZ groups).
} 


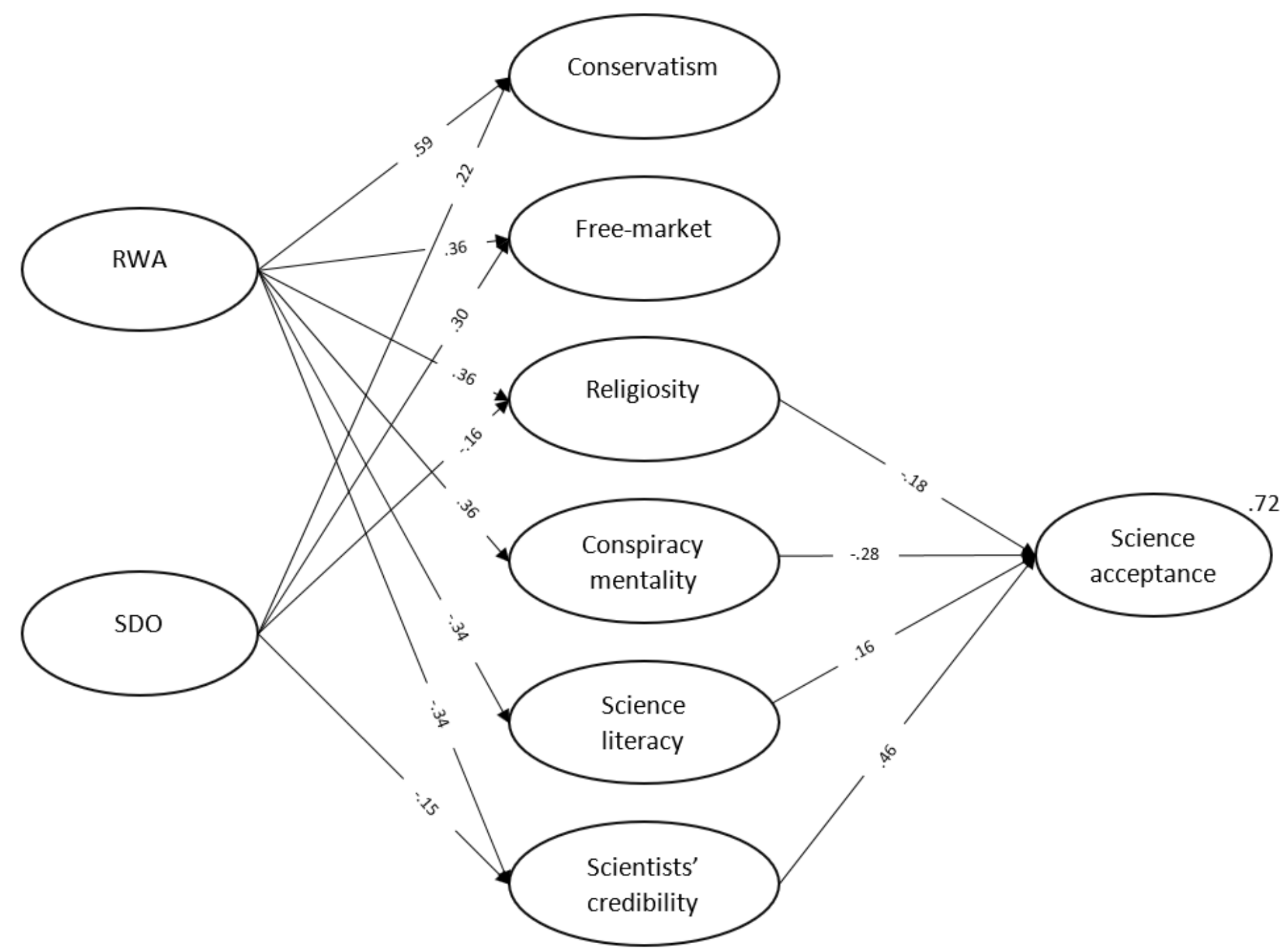

Figure 6.4. Mediators of the effects of RWA and SDO on a second order latent science acceptance variable (Study 6). Non-significant and small $(<|.10|)$ paths, covariances, and age and gender covariates not shown (estimates for all parameters are reported in Appendix H). 
Turning our attention to the mediating variables in the model, I report that religiosity and conspiracy mentality are negative predictors of science acceptance while science literacy and perceived credibility of scientists are positive predictors. A notable difference between the results of this model and those in Study 5 is that religiosity is a significant negative predictor of science acceptance in the NZ sample but not the US sample.

Considering the total, direct and indirect effects of ideological attitudes on science acceptance (Table 6.10), we find that both RWA and SDO and have a significant, negative total effect on science acceptance, almost fully mediated by the other variables in the model. RWA does not have a significant direct effect while SDO does have a small, negative direct effect $(\beta=-.05, b=-.06,[-.07,-.02]$; not shown in Figure 6.4). Thus, I report that New Zealanders who are more authoritarian or more socially dominant are more likely to disagree with the mainstream scientific consensus, and these effects are explained, in part, by lower trust in science and scientists.

Table 6.10

Indirect, direct and total effects of RWA and SDO on overall science acceptance (Study 6)

\begin{tabular}{|c|c|c|c|c|}
\hline Independent variable & Mediator & $\beta$ & $b$ & $95 \mathrm{CI}$ \\
\hline \multirow[t]{8}{*}{ RWA } & Religiosity & -.08 & -.06 & {$[-.11,-.06]$} \\
\hline & Conservatism & -.02 & -.02 & {$[-.05, .01]$} \\
\hline & Free market ideology & -.01 & -.01 & {$[-.03,0]$} \\
\hline & Conspiracy mentality & -.13 & -.10 & {$[-.15,-.11]$} \\
\hline & Scientists' credibility & -.21 & -.16 & {$[-.24,-.18]$} \\
\hline & Science literacy & -.07 & -.05 & {$[-.09,-.05]$} \\
\hline & Direct effect & .01 & .01 & {$[-.05, .07]$} \\
\hline & Total effect & -.52 & -.39 & {$[-.6,-.44]$} \\
\hline \multirow[t]{8}{*}{ SDO } & Religiosity & .02 & .03 & {$[.02, .03]$} \\
\hline & Conservatism & .00 & -.01 & {$[-.01, .001]$} \\
\hline & Free market ideology & -.01 & -.01 & {$[-.02, .00]$} \\
\hline & Conspiracy mentality & -.02 & -.02 & {$[-.02,-.01]$} \\
\hline & Scientists' credibility & -.05 & -.07 & {$[-.07,-.04]$} \\
\hline & Science literacy & .00 & .00 & {$[-.002, .01]$} \\
\hline & Direct effect & -.05 & -.06 & {$[-.07,-.02]$} \\
\hline & Total effect & -.11 & -.13 & {$[-.14,-.07]$} \\
\hline
\end{tabular}

Standardized $(\beta)$ and unstandardized $(b)$ effects shown. Bias-corrected bootstrapped $95 \%$ confidence intervals (5,000 samples) are for $b$ values. Bold values denote significant effects (confidence interval does not include zero). 


\subsection{DISCUSSION}

Several studies report that RWA and SDO predict climate change denial (Stanley \& Wilson, 2019), but the role of these attitudes in beliefs about other scientific issues has not been investigated. The studies in this chapter show, for the first time, that RWA predicts rejection of the mainstream scientific consensus on GM food, vaccination, evolution and fluoride. SDO also predicts rejection of the scientific consensus on vaccination and, marginally, fluoridation. I also replicate previous research regarding climate change; across all three studies RWA and SDO predict rejection of climate science. In sum, individuals who are more authoritarian or more accepting of inequality are less likely to align their views with the scientific consensus on socially debated issues. Thus, the current research adds to the growing literature examining the ideological antecedents of rejection of science (Hornsey \& Fielding, 2017; Lewandowsky \& Oberauer, 2016; Rutjens et al., 2018).

The current research also offers some insight into why authoritarian and socially dominant individuals are more likely to reject science. In Studies 5 and 6, I used SEM to examine potential paths by which RWA and SDO might influence beliefs about specific scientific issues in US and New Zealand samples. Specifically, this research examined several previously identified predictors of rejection of science (such as political ideology and religiosity) and situated these within the larger theoretical framework of the DPM (Duckitt \& Sibley, 2016). These predictors were incorporated as parallel mediators in a model seeking to disentangle the paths which lead to high RWA and SDO individuals to rejecting science. The model explained substantial variation in beliefs about all five scientific issues examined and revealed the heterogeneity of influences across scientific domains. For example, although political ideology correlated with rejection of science in every domain, the model revealed that, accounting for other predictors, political conservatism only predicted climate change denial. Across Studies 5 and 6 we observe a consistent pattern: perceived credibility of scientists - predicted by both RWA and SDO — is associated with rejection of science in every domain examined.

When considering the separate effects of ideological attitudes, we find that both RWA and SDO explain unique variance in rejection of science. This indicates RWA and SDO are separate, but related, antecedents of beliefs about scientific issues, and parallels previous research applying the DPM in the spheres of prejudice and political beliefs (i.e., there are two separate motivational paths influencing ideology and socio-cultural beliefs; Duckitt \& Sibley, 2016). However, the two attitudes are not identical in their associations with rejection of 
science. High SDOs are not more likely to reject GM food or evolution science-in fact they were more likely endorse evolution science in the NZ sample (by virtue of lower religiosity among high SDOs). Thus, while RWA is a consistent predictor of rejection of science, the effects of SDO depend on the domain in question. In all samples both RWA and SDO were significantly correlated, which highlights the need to consider both constructs simultaneously in future research examining their relationship with rejection of science. Studies which focus on just, say, RWA may erroneously attribute effects to authoritarianism when in fact an association is due to RWA's overlap with SDO (see also Milfont et al., 2013, Study 3).

The results also align with previous research on RWA and SDO in predicting sociopolitical attitudes (reviewed in Section 3.3.3). Both RWA and SDO explain unique variance in political ideology, free market ideology and religiosity. In the case of conspiracy mentality, RWA is a robust predictor of conspiracy thinking but SDO is not. ${ }^{23}$ This conflicts with the Wilson and Rose (2014) but is consistent with other studies (see Grzesiak-Feldman, 2015).

There are some differences between the results of the US and NZ models which I will take a moment to reflect on. For example, free market ideology predicted acceptance of GM food science in the NZ (but not US) sample. This difference may be attributable to the level of perceived government involvement and regulation regarding GM crops. The US has adopted a "quite permissive approval policy" (Lucht, 2015, p. 4257) for GM crops while the more restrictive $\mathrm{NZ}$ regulatory regime has been accused of holding back agricultural innovation and economic growth (Fritsche et al., 2018). Thus, NZ free market adherents may be more attuned to the business impacts of regulatory restrictions and more supportive of GM food generally. We also find that science literacy was a significant predictor of acceptance of evolution and fluoridation science in the NZ sample but not US sample. This may be attributable to differences in sample size between the studies and power to detect a significant effect. $^{24}$

Although we find that most of the effects of RWA and SDO on rejection of science were explained by ideological and science-specific mediators, there were some direct effects of ideological attitudes on rejection of science. In both the NZ and US samples, SDO had a direct effect on rejection of climate science, and I report a direct effect of RWA on rejection

\footnotetext{
${ }^{23}$ SDO had no significant effect on conspiracy mentality in the US model and only a weak $(\beta=.08, p<.001)$ effect in the NZ model (not shown in Figure 6.3 due to small size).

${ }^{24}$ In fact, the magnitude of the estimated regression coefficient for the effect of science literacy on fluoridation was similar between samples, but not significant in the US model $\left(\beta_{U S}=.11, p=.06 ; \beta_{N Z}=.10, p<.001\right)$.
} 
of vaccine science in the NZ sample. ${ }^{25}$ What factors (not included in the model) might explain these relationships? In the case of SDO, one possible explanatory variable absent from the models is environmental values. High SDOs are less likely to value the environment, which is in turn associated with climate change denial (Jylhä \& Akrami, 2015; Milfont et al., 2013). In the case of RWA and anti-vaccine sentiments, a possible mediator unexamined in the current research is purity concerns. Within Moral Foundations Theory, the foundation of purity/sanctity captures an abhorrence of the impure, disgusting and unnatural (both in a physical and spiritual sense; J. Graham et al., 2011). Separate studies have reported a positive association between the moral foundation of purity and both RWA and antivaccine beliefs (Federico, Weber, Ergun, \& Hunt, 2013; Rossen, Hurlstone, Dunlop, \& Lawrence, 2019; Rutjens et al., 2017). Future studies should merge these findings and investigate if purity concerns underlie the unexplained association between RWA and rejection of vaccine science reported here.

To explore a more generalised rejection of science, I also modelled a second-order latent variable representing a general tendency to agree with the mainstream scientific position. In this model I find that both RWA and SDO have an overall negative association with the endorsement of the mainstream scientific position on socio-scientific issues. These effects are mediated by a perception of scientists as trustworthy and credible and, to a lesser extent, conspiracy beliefs, general scientific knowledge, political ideology (US only), free market ideology (US only) and religiosity (NZ only). The divergent results between samples suggest that authoritarian and social dominant opposition to science is more intertwined with politics in the US and religion in NZ. This finding fits with research reporting that scientific issues appear to be more politicised in the US compared to other developed nations (e.g., climate change: Hornsey et al., 2018a; evolution: Miller et al., 2006).

\subsubsection{Limitations}

Overall, the results of Studies 4, 5, and 6 make a strong case for the role of RWA and SDO in rejection of science, with relatively consistent results across three very different samples, including a very large non-representative public New Zealand sample. Studies 5 and 6 also employed modelling techniques which accounted for multivariate non-normality and allowed simultaneous estimation of multiple indirect effects. However, I must acknowledge

\footnotetext{
${ }^{25}$ There were also several smaller $(\beta<|.10|$ ) direct effects of RWA on rejection of science in Study 6 (See Table H.2, Appendix H), which, for brevity, I will not discuss here.
} 
several limitations. Firstly, all three studies were cross-sectional in nature, limiting the causal inferences that can be made regarding the relationships between ideological attitudes and rejection of science. Future experimental and longitudinal work is required to confirm the causal direction assumed in these models. Secondly, while the research covered samples from both the US and NZ, both are WEIRD populations (Western, educated, industrialized, rich, and democratic; Rad, Martingano, \& Ginges, 2018). Thus, we must exercise caution in generalising the current results to different contexts, such as developing or culturally collectivist countries.

As the first set of studies to investigate the role RWA and SDO in rejection of science, the variables included in the models in this chapter are relatively broad constructs. For example, RWA and SDO were treated as single variables in this chapter but could be decomposed into sub-dimensions. Some versions of the RWA scale (e.g., Duckitt et al., 2010) can be broken down into three separate factors: aggression, conservatism and traditionalism. Likewise, Ho et al. (2015; following Jost \& Thompson, 2000) theorise that SDO consists of two sub-dimensions, dominance and egalitarianism, which differentially predict some prejudicial attitudes (and climate change beliefs; Stanley, Wilson, Sibley, \& Milfont, 2017). The same reasoning applies to the mediating variables included in the model. The measure of general political ideology used in this chapter combined social and economic views, which could be considered separately (cf. Carl \& Cofnas, 2016). The measure of religiosity did not differentiate between different religions or denominations (cf. Steensland et al., 2000). The generic conspiracy ideation examined in this thesis may encompass several separate dimensions of suspicious thinking, including: concerns about personal health and liberty and suspicions of government malfeasance (Brotherton, French, \& Pickering, 2013); or conspiracy theorising and 'rational scepticism' (Stojanov \& Halberstadt, 2019). The science literacy scale only covered knowledge of science facts; more nuanced versions also include understanding of theoretical concepts in science (e.g. the use of control groups; Drummond \& Fischhoff, 2017). Finally, Hartman et al.'s (2017) measure of scientists' credibility combined trustworthiness and perceived expertise. These elements could be separated and investigated independently (cf. Morgan, Collins, Sparks, \& Welch, 2018). Suffice to say, Studies 5 and 6 investigated the relationships between broadly conceptualised, high-level constructs. Any of these could, and should, be broken down and more narrowly examined as predictors of rejection of science in future research, though at the risk of making models such as those reported here even more complicated. 


\subsubsection{Trust in scientists}

The combination of predictors for each rejection of science issue offers rich insights for researchers focusing on beliefs and attitudes in each domain. However, due to space, I shall focus the remaining discussion here on the consistent mediator identified across all issues in both Studies 5 and 6: perceived credibility of scientists. As noted earlier, Hartman et al.'s (2017) Credibility of Science measure can be considered a measure of trust in scientists given the scale's description and content (see Section 3.2.1). I hypothesised that RWA and SDO would be linked with distrust of science and scientists as well rejection of specific scientific claims (see Section 3.3.3), and my results show this to be the case. I theorised that this distrust of science arises from high SDOs perceiving scientists as threatening hierarchical social structures, and high RWAs perceiving scientists as violating moral norms. This reasoning remains speculative. Further research is required to find out how high RWA and SDO individuals perceive the actions, motives and intentions of scientists and how this in turn affects the level of trust they place in science.

An important question not addressed in the current research is who people think of when considering 'scientists'. People perceive different scientific fields as more or less precise (and therefore credible) and these perceptions differ by political ideology (Broomell \& Kane, 2017). McCright et al. (2013) report that conservatives (vs liberals) are less trusting of 'impact' scientists, "those in the service of understanding human impacts on the environment and human health," but more trusting of 'production' scientists undertaking "scientific endeavors in the service of economic production" (p. 2). Given conservatives' overall lower trust of non-descript 'scientists' (Gauchat, 2012), we might assume that impact scientists are the category that comes most easily to mind when participants are asked to evaluate scientists. Future research should investigate how mental representations of 'scientists' influence evaluations of science and scientists.

The finding that both RWA and SDO predict negative attitudes to scientists should also be considered in the context of previous research applying the DPM to prejudice. Duckitt and Sibley (2007) report that attitudes towards a range of social groups appear to cluster into three distinct categories - dangerous, derogated and dissident-with prejudice towards these broad groups differentially predicted by RWA and SDO. High RWA (but not SDO) individuals show greater prejudicetowards socially threatening, but not subordinate, 
dangerous groups such as terrorists or drug dealers. ${ }^{26}$ High SDO (but not RWA) individuals show greater prejudice towards derogated groups who are socially subordinate or compete for social status and resources, such as ethnic minorities, immigrants, and people with disabilities. Both high RWA and high SDO individuals show greater prejudice towards dissident groups "dissenting from, challenging, or opposing mainstream norms and values" (p. 120), such as feminists, protestors and people who 'cause disagreement'. This three-factor model of prejudice has been confirmed in subsequent research (Asbrock, Sibley, \& Duckitt, 2009; Sibley, Harding, Perry, Asbrock, \& Duckitt, 2010).

Examining prejudice towards scientists alongside other social groups could aid in understanding what drives the association between RWA, SDO and distrust of scientists. An example of this can be seen in the work of Cantal, Milfont, Wilson, and Gouveia (2015) who incorporate 'politicians' as social group alongside previously-examined groups in Duckitt and Sibley's (2007) three-factor model of prejudice. The authors report that, in Brazilian samples, politicians sit within the dangerous category - predicted by RWA but not SDO (possibly due to the corrupt behaviour of politicians in the Brazilian context). The fact that both RWA and SDO predict negative attitudes towards scientists in the current research suggests that scientists may sit in the dissident category (alongside atheists, feminists, environmentalists, protestors, and 'people who criticise'; Cantal et al., 2015). Such a conclusion fits well with claims that scientists (at least in an academic setting) are 'subversive' or 'the critic and conscience of society' (Grace, 2010; Sidanius, Pratto, Martin, \& Stallworth, 1991). Future research should follow in the footsteps of Cantal et al. (2015) and confirm the place of scientists as a social group within the three-factor model of prejudice.

\subsubsection{Motivated reasoning}

The findings also temper the assumed role of motivated reasoning in rejection of science. The negative relationship between ideologies and science acceptance is often attributed to motivated reasoning (Druckman \& McGrath, 2019). That is, people are motivated to deny specific scientific findings that conflict with ideological values. The current studies show that this is the case for some issues, but trust also plays a key role, with distrust of scientists associated with rejection of science for every issue examined, even when ideology is taken into account. As noted by Druckman and McGrath (2019), an accuracy

\footnotetext{
${ }^{26}$ Prejudice was operationalised using an 'affective thermometer' rating scale in which participants rated the 'warmth' of their feelings towards groups (see Duckitt \& Sibley, 2007, p. 118)
} 
motivated individual who distrusts scientists may 'rationally' disregard the claims of the scientific community in the pursuit of an accurate conclusion. Thus, the assumption that all rejection of science is the product of ideologically motivated reasoning should be treated with scepticism.

There is a further point to consider regarding trust in science and motivated reasoning. Most studies (including the current research) assume trust in scientists to be a causal driver of rejection of science, but I acknowledge a case can be made that the direction of influence is bidirectional. Where scientific findings conflict with ideology, people may engage in motivated reasoning, discounting the credibility of scientists generally, allowing them to maintain their prior beliefs more comfortably (Cook \& Lewandowsky, 2016; Kahan et al., 2011). Ultimately, experimental and longitudinal research is required to elucidate the causal direction of links between perceptions of scientists generally and beliefs about specific issues.

\subsubsection{Practical implications for science communication}

The results of the studies in this chapter also offer some practical insights regarding effective science communication, providing an indication of 'what matters' for different issues. Understanding how trust, values and ideological beliefs influence peoples' opinion on specific scientific issues is critical to public engagement (National Academies of Sciences, Engineering, and Medicine, 2016a). The studies in this chapter highlight the complexity of the beliefs associated with a variety of publicly debated issues. By examining several overlapping constructs, we can disentangle the different predictors and shed light on realworld discussions of the drivers of rejection of science. For example, commentators in New Zealand and the US have accused both the political left and right of taking an anti-science stance (Hanna, 2016; Louisson, 2016; Mooney, 2012; Tierney \& Stossel, 2018). While my research shows conservative political ideology is correlated with rejection of science across all five issues, the SEM results indicate that focusing on politics specifically doesn't have much relevance outside of climate science. Political conservatism is unrelated to beliefs about other issues once further predictors are considered. Mis-informed communication efforts which sought to focus on or frame material to appeal to liberals or conservatives on issues such as vaccination or GM food might be missing the mark.

Scientific knowledge, as indexed by a short quiz, was a significant predictor of attitudes towards vaccination and GM food (and evolution and fluoridation in Study 6 only). This again indicates that rejection of science is not purely ideological; there appear to be some 
cognitive constraints as well. While the effects of science literacy were limited, this does suggest that educational efforts focusing on information and knowledge may have some benefit in shifting personal beliefs about scientific issues regardless of ideological conflicts (see also McPhetres, Rutjens, Weinstein, \& Brisson, 2019; Ranney \& Clark, 2016). That RWA negatively predicts science literacy in both US and NZ samples is interesting but difficult to interpret in the current context. It is possible that poorer cognitive ability underpins both factors, as previous research suggests that cognitive factors play a role in authoritarian beliefs (Jost, Kruglanski, et al., 2003; Onraet et al., 2015).

By drawing on more nuanced analyses of the ideological predictors of rejection of science, science communicators can craft messages to be ideologically congenial to a given audience for a given issue and sidestep potential conflicts (Hornsey \& Fielding, 2017; Jamieson \& Hardy, 2014). For example, organisations seeking to promote water fluoridation to a sceptical audience should be aware of the fact that limited scientific knowledge, conspiracy thinking, and a distrust of scientists are all associated with fluoridation opposition. Thus, efforts to engage such an audience might involve some basic description of water fluoridation and evidence of its efficacy (addressing lower science knowledge), presented in collaboration with a trusted non-scientist source, such as a community leader. Reflecting on the need for diverse sources of scientific information in such situations, Kahan (2010) writes, "People feel that it is safe to consider evidence with an open mind when they know that a knowledgeable member of their cultural community accepts it" (p. 297). Engagement efforts could also incorporate some communication techniques aimed at overcoming suspicions of a conspiracy, such as validating general conspiracy belief while debunking fluoridation conspiracies, highlighting potential counter-conspiracies (e.g. bottled water companies seeking to profit from fear of fluoride), or increasing individuals' sense of personal control (Bolsen \& Druckman, 2018b; Hornsey \& Fielding, 2017; Whitson, Kim, Wang, Menon, \& Webster, 2019).

I must also address the challenge of general distrust of science, which is more prevalent among high RWA and SDO individuals. While some degree of scepticism regarding science is desirable (Lewandowsky, Mann, Brown, Friedman, \& Friedman, 2016), outright distrust of scientists may lead to beliefs that are potentially harmful to oneself and others (e.g. the belief that vaccines are dangerous or ineffective), as indicated by the results of the studies in this chapter. Possible interventions for increasing public trust in scientists include: increasing the transparency of scientific research, engaging in two-way dialogue with non-scientist 
stakeholders and improving science education (Elliott \& Resnik, 2014; Lewandowsky, Cook, et al., 2017; National Academies of Sciences, Medicine, and Engineering, 2015).

How might scientists or policymakers specifically target individuals whose scepticism of science is grounded in RWA or SDO? A good starting point would be to focus on how on the work of scientists aligns with the motivations underlying these ideological attitudes. Recall that Duckitt and Sibley (2016) describe RWA and SDO as the attitudinal expression of motivational goals. High RWAs are motivated by goals of collective security, control, and stability. High SDOs are motivated by goals of power, dominance, and superiority. Framing the work of scientists to align with these goals may increase trust among the similarly motivated. Authoritarians' view of science might be softened by presenting scientists as protecting the health and safety of communities, neutralising threats, or mitigating the destabilising impacts of environmental change. An alternative approach would be to highlight the role of scientists as acting on behalf of established authorities (e.g. through direct government employment or funding), courting the authoritarian submission component of high RWAs’ beliefs. Scientists who identify as religious might even describe themselves as 'doing God's work'. For socially dominant groups, trust might be gained by emphasising the role of science in maintaining national dominance on the world stage through military technology or technological innovations. These approaches and their impact on trust in scientists among high RWA and SDO individuals should be examined in future research. Experiments applying the DPM to message framing in the political sphere offer a useful template for such studies (Crawford, Brady, Pilanski, Erny, \& Erny, 2013).

Scientists seeking to build trust might also emphasise shared values when engaging with communities likely to endorse authoritarian or social dominance values. For example, scientists communicating with fundamentalist religious groups might highlight their own religious beliefs (if applicable; Cloud, 2016), or stress the importance they place on tradition or 'keeping things the way they are' (e.g., Baldwin \& Lammers, 2016). A similar approach could also be applied in communicating with typically high SDO audiences (e.g. lawyers or business leaders; Pratto et al., 1997). For example, scientists could highlight the competitive, hierarchical and meritocratic aspects of the science sector as a way of indicating shared values. While these suggested approaches might be useful in specific contexts, I would not recommend a larger scale effort to emphasise these values in portrayals of science and scientists in the wider media. Such a shift would be undesirable as it could lead to more 
distrust of scientists among the wider public who do not endorse authoritarian or socially dominant values.

\subsubsection{Conclusions}

Considering the role of factors such as politics, religion and conspiracy thinking in rejection of science, my results echo the findings of Rutjens et al. (2017) who conclude that "...different forms of science acceptance and rejection have different ideological roots, although the case could be made that these are generally grounded in conservatism" (p. 396; emphasis in original). Here the authors refer not to political conservatism specifically but a more general orientation towards valuing tradition, stability and hierarchy. My finding that ideological attitudes predict rejection of science across a range of issues validates Rutjens et al.'s second point. In this respect I would argue that RWA and SDO represent this grounding orientation, capturing deeper ideological attitudes regarding tradition, hierarchy and authority upon which ideologies (such as politics) may be predicated (Duckitt \& Sibley, 2009; M. S. Wilson \& Sibley, 2013).

As a final note, I remind the reader that RWA and SDO are stable, but not immutable attitudes. Though empirical evidence is limited, RWA and SDO are theorised to increase in response to threatening and competitive environments (Duckitt \& Fisher, 2003; Sibley \& Duckitt, 2013). Following from this we would expect that events such as terrorist attacks, wars, political instability and financial crises (all of which have occurred post-2000) would have an indirect eventual impact on public opinion regarding in science. Increases in RWA and SDO can drive a rise in political conservatism, religiosity and conspiracy thinking as well as undermining trust in scientists - all ultimately leading to more disagreement over scientific findings. There is a counter-point to this: if people perceive the world as a less threatening and competitive place, society as whole may become more accepting of scientific evidence. 


\section{Chapter 7: Final discussion and conclusions}

In this final chapter, I review the research presented in this thesis and offer some thoughts on future research.

\subsection{THESIS SUMMARY}

The central aim of this thesis was to investigate why people hold beliefs in opposition to scientific consensus. As reviewed in Chapter 2, people differ in their views on issues where a strong scientific consensus exists; some people disagree that, for example, human activities are causing climate change, or that vaccines are effective in preventing disease. Understanding how such beliefs form and change is critically important, as beliefs about contested issues like climate change and vaccination can have wide-ranging impacts on the environment and public health.

One possible answer to the question of why people disagree with a scientific consensus is that people simply don't know there is a consensus. If you can convince someone that most scientists agree on a given claim, will that person align their own beliefs with that of scientists? This chain of influence is captured in the GBM, a model which places perceptions of consensus as a causal driver of personal beliefs regarding scientific issues. Two studies in this thesis investigated the GBM. The first (Study 1) focused on the effects of exposure to information about scientific consensus regarding either human-caused climate change or the safety of GM food. Message-induced increases in perceived consensus are associated with greater reported belief that humans are causing climate change and that GM food is safe to eat. Thus, I report some experimental evidence for the GBM in the context of two debated scientific issues.

However, a second study examining the relationship between perceptions of consensus and personal beliefs (Study 2) found that the former did not prospectively predict the latter over time, as would be predicted by the GBM. While this result did not support the GBM, an analysis published by van der Linden et al. (2018a) presents a different result in a larger, more representative US survey using similar measures. The authors report that perceptions of consensus do predict later belief in human-caused climate change. Thus, the results reported in Study 2 appear not to generalise to US adults. However, both Study 2 and van der Linden et al. (2018a) report an inverse effect of personal beliefs on perceptions of consensus, 
indicating perceived consensus should not be considered an absolute precursor to personal beliefs, nor communicating consensus a silver bullet. That is, our personal beliefs may also skew our perceptions of the level of scientific agreement on socially relevant scientific issues.

The somewhat conflicting results of Study 1 and Study 2 do converge on one finding relevant to the rest of the thesis: perceptions of consensus alone do not fully explain rejection of science, indeed, perceived scientific agreement accounts for less than $11 \%$ of variance in acceptance of climate science in Study 2. ${ }^{27}$ The remaining studies in the thesis examine alternative explanations for why people hold views in conflict with a scientific consensus. Moving beyond a simple lack of awareness of a consensus, I investigated how ideologies, coherent and stable belief systems, are tied to, and may underlie, rejection of science.

Study 3 took a novel approach to exploring the role of political ideology in rejection of science, using the selection of information sources as a proxy for political and scientific beliefs. Analysing a very large dataset drawn from the social media platform Twitter, I report that the following of conservative (vs liberal) NZ political accounts is associated with following accounts sharing counter-scientific information regarding climate change and vaccination and is very slightly associated with acceptance of the scientific consensus on GM food safety. Thus, in agreement with previous survey research, political ideology is linked with rejection of science on some issues in the context of social media. That is, who people choose to follow on twitter appears consistent with what people say about themselves in survey research - at least regarding climate change and vaccination. The weak, negative association between conservative ideology and following anti-GM accounts is an interesting exception which I will return to when comparing the results of studies in the section below.

Political ideology is just one of several ideological factors implicated in the rejection of science. Studies 4, 5 and 6 investigated a wider range of ideological predictors, examining the relative importance of different ideologies in relation to beliefs about five separate scientific issues: climate change, GM food, vaccination, fluoridation and evolution. Here I also included scientific knowledge and trust in scientists as further predictors of rejection of science on these issues. I also placed all these predictors in the wider context of the DPM, a well-established psychological model which explains a range of socio-political outcomes. I

\footnotetext{
${ }^{27}$ van der Linden et al. (2018a) report slightly larger (cross-sectional) correlations between perceived consensus and personal belief in climate change, indicating perceived consensus explains $14-16 \%$ of variance in personal belief ( $r$ s .37-.41).
} 
investigated the role of two key constructs in the DPM, RWA and SDO, in predicting rejection of science and established antecedents of rejection of science.

Studies 5 and 6 carry several strengths over previous research. They employ SEM, which accounts for measurement error and allows estimation of multiple indirect effects (compared to OLS regression approaches; cf. Drummond \& Fischhoff, 2017; Rutjens et al., 2017). These studies use data from non-student adult samples and Study 6, in particular, draws on a very large (though non-representative) sample of New Zealanders. Examining rejection of science in just one domain would have been a worthwhile goal, but these studies cover not one, but five high-profile scientific issues, offering an unprecedented opportunity to contrast and compare the predictors of different contested scientific beliefs.

Unsurprisingly, the results of these studies present a very complex map of the relative influence of the different attitude roots of rejection of science. However, several clear conclusions can be drawn. These studies show, for the first time, that RWA and, to a lesser extent, SDO predict rejection of science across a range of issues. We also find that the mediators of these effects vary depending on the scientific claims in question. For example, conservative political ideology explains some of the association between RWA and climate change denial, but not the association between RWA and scepticism of GM food. Trust in scientists emerges as a robust predictor of rejection of science across all issues and is one of the strongest mediators of the relationships between RWA, SDO and rejection of science. The research in these final studies breaks important new ground in identifying broad psychological predictors of rejection of science across scientific domains and explaining their association with beliefs about specific scientific issues.

\subsection{REFLECTIONS}

The studies in this thesis all tackle explanations for why people disagree with scientifically valid claims. I must acknowledge a pivot between the first two studies and the remaining four, but there is a logical progression. We start with common-sense explanations (a lack of awareness) and move through to more complex and less obvious explanations, ending with a focus on stable beliefs about the structure of society which, on the face of it, appear to have nothing to with specific claims that, say, $\mathrm{CO}_{2}$ warms the atmosphere or fluoride protects teeth.

The shift from perceptions of consensus to ideological explanations for rejection of science was prompted in part by the conflicting results of Study 1 and 2 . While I could have 
continued to investigate the consensus-belief link further, I felt that recent work in identifying the disparate ideological drivers of rejection of science (e.g. Rutjens et al., 2017) presented an opportunity to contribute more meaningfully the literature. In particular I saw the possibility that RWA and SDO may predict rejection of science across a range of issues, given their association with previously established attitude roots of rejection of science (Hornsey \& Fielding, 2017; Rutjens et al., 2017), and recent research linking RWA and SDO with climate denial (Jylhä \& Akrami, 2015; Milfont et al., 2013). This hunch proved correct, with Studies 4, 5 and 6 revealing, for the first time, that both RWA and SDO explain variation in beliefs about a variety of contested issues with these effects mediated by a range of factors, most critically trust in scientists.

While this approach has resulted in a thesis narrative with a few twists and turns, I do not regret the decision to start my research with a focus on the GBM. Working with a simpler model at the beginning of the research journey gave me an opportunity to build my research and analysis skills to the point where I was confident taking on a more comprehensive examination of the ideological predictors of rejection of science across a range of issues.

\subsection{FINAL CONSIDERATIONS AND FUTURE DIRECTIONS}

The discussion sections of the preceding three chapters have already offered some suggestions for future research relevant to the studies presented. Here, I will note several points where the studies in this thesis intersect and suggest avenues for further research.

\subsubsection{Extending the GBM}

Trust in scientists is a key predictor of rejection of science across all issues examined in Studies 5 and 6. This construct also has relevance for the GBM examined in Studies 1 and 2: does trust moderate the relationship between perceived consensus and personal beliefs? One might expect that awareness of scientific agreement would have less impact on personal beliefs among people who do not trust scientists. Indeed, as reviewed in Section 3.1.3, the GBM is based on the argument that people use consensus information as a guide for their own beliefs in part because they rely on the heuristic rule-of-thumb 'experts can be trusted' (van der Linden et al., 2019).

The role of trust as a moderator has been examined in recent studies focussing on the GBM (Chinn et al., 2018; Dixon, 2016; Kobayashi, 2018). Methodological differences make direct comparisons between studies difficult but results generally indicate that trust in scientists has little to no impact on the strength of the association between perceived 
consensus and personal beliefs, or on the influence of consenus messages (although see Pasek, 2018). Reflecting on this research, van der Linden et al. (2019) caution against unnecessary additions to the GBM, noting, "it should be illustrated that making the model more complex by adding individual difference moderators is worth the trade-off by corresponding increases in model fit, explanatory, or predictive power" (p. 56).

Acknowledging this, further research should still clarify the moderating role of trust in scientists in the GBM. The idea that people who trust scientists and people who distrust scientists are equally receptive to a scientific consensus is difficult to marry with the theoretical underpinnings of the GBM. If this is the case, more work will need to be done to understand why people who distrust scientists still update their beliefs in alignment with scientific consensus information.

Studies 1 and 2 in this thesis focused primarily on the link between perceived consensus and belief in human-caused climate change. However, the GBM does extend beyond this, positing that, in the context of climate change, changes in perceived consensus have a 'cascading effect', also influencing concern over climate change and support for climate mitigation policies (van der Linden, Leiserowitz, et al., 2015; van der Linden et al., 2019). These downstream attitudes are arguably more important in terms of society mitigating and adapting to a warming world. In extending the GBM to other contested scientific issues, researchers should include similar constructs, such as support for policy and actual or intended behaviour.

Finally, while the GBM has been applied to a range of rejection of science issues including vaccination and GM food (e.g., Dixon, 2016; van der Linden, Clarke, et al., 2015), including further topics may help establish the boundaries of consensus messaging. For example, rejection of the theory of evolution has persisted for more than a century in the face of an ever-growing body of evidence. Indeed evolution appears to be the issue for which people are most likely to acknowledge, yet still reject a scientific consensus (Pasek, 2017). Does highlighting the scientific consensus have any impact on people who reject evolution science?

\subsubsection{Attitude roots and rejection of science on social media}

Study 3 used the following of different Twitter accounts as indicators of political and scientific views, identifying a link between political ideology and rejection of science on some issues. Studies 5 and 6 highlighted the unique effects of other ideological variables 
which also correlated with political ideology (e.g. religiosity). Future research could attempt to incorporate these further variables in models predicting the following of science sceptical accounts as seen in Study 3. For example, are people who follow accounts endorsing religious, free market, or conspiracy theory views more likely to follow science-sceptic accounts? Researchers could identify the relative importance of different ideologies by combining the following of several different types of accounts (e.g. conservative, conspirarcy) into models predicting the following of science sceptic accounts or more complex network analyses.

Further research using Twitter follower networks could also be combined with survey-based studies like those presented in Chapters 5 and 6. For example, participants could provide their Twitter username in a survey, allowing data on which accounts they follow could to be merged with their self-report data (assuming informed consent and ethical safegards; Rivers \& Lewis, 2014). This would validate the use of Twitter data as an indicator of attitudes towards scientific issues (as has already been done with political ideology; Eady et al., 2019). Such research might help explain the inconsistency of results regarding political ideology and anti-GM attitudes across studies. In Study 3 we find that a continuous measure of political conservatism is weakly, negatively associated with following anti-GM accounts, but in Study 6 self-reported conservatism is positively correlated with anti-GM attitudes. This may be attributable to differences between NZ twitter users and NZ survey participants, or it may be the case that the following anti-GM accounts is not a good indicator of personal beliefs about GM food. Combining self-report and social media data would clarify the situation.

\subsubsection{Explaining the unexplained (variance)}

The models in Studies 5 and 6 explained a respectable amount (between a quarter and half) of the total variation in people's beliefs about scientific issues, greater than that seen in similar studies (e.g., Rutjens et al., 2017). ${ }^{28}$ However, that means there is still a substantial degree of unexplained variance. What might account for this?

\footnotetext{
${ }^{28}$ Specifically, Rutjens et al. (2017) regressed climate, GM food and vaccine beliefs on to the five moral foundations, political ideology, religious identity, religious orthodoxy, 'faith in science' and science literacy. Adjusted $R^{2}$ values for these (OLS) models were, $.37, .19$ and .15 respectively. These can be compared with $R^{2}$ values of $.50, .34$ and .29 for the same dependent variables in the Study 6 SEM (the climate change and vaccine measures were identical to those used by Rutjens et al. but this study used a different measure of GM food beliefs).
} 
One further variable which may explain variation in these models is perceptions of consensus. However, the inclusion of this variable in the models would raise questions about causal paths. The results of Study 2, as well as van der Linden et al. (2018a) suggest a perceived consensus may be influenced $b y$, rather than solely influence, personal beliefs. Kahan (2017) suggests that self-professed belief in human-caused climate change and belief that it is supported by a scientific consensus are both indicators of a more general underlying affective attitude towards climate change. That is, questions about perceived consensus and questions about personal beliefs may be essentially measuring the same thing (indeed, some studies have combined them into a single index; e.g, L. Feldman, Maibach, Roser-Renouf, \& Leiserowitz, 2012). I believe that continuing research on the GBM and the efficacy of consensus messaging is necessary but including perceptions of consensus in cross-sectional models predicting rejection of science may be problematic due to the potentially bidirectional causal relationship.

As noted in the Chapter 6 discussion, environmental values predict climate change denial, and I suspect a measure of such values (e.g. the New Ecological Paradigm scale; Stern, Dietz, \& Guagnano, 1995) would explain variance in climate beliefs over and above the predictors included in Chapter 6 (Milfont et al., 2013; Stanley, Wilson, \& Milfont, 2017). It might also predict rejection of science in other domains; there is some evidence that environmental values predict GM food beliefs (Hall \& Moran, 2006; Slimak \& Dietz, 2006).

A widespread belief that 'natural is better' might also underpin rejection of science. GM food, vaccination and fluoridation, all represent modern technological interventions that could be perceived as interfering with the natural order. All things being equal, people tend towards preferring natural (vs. 'artificial') foods, medicine, and even hazards, but there is variation within populations (Rozin et al., 2004; Rudski, Osei, Jacobson, \& Lynch, 2011). Some people are more likely to subscribe to the 'naturalistic fallacy', the belief that because something is natural it must be better (Lull \& Scheufele, 2017). Psychologist Paul Rozin offers a useful comparison in the context of rejection of science: "natural is treated as a basic good thing, not subject to evidence, somewhat like belief in God for those who believe in God" (Hale, 2018). Individual difference measures of preference for 'naturalness', or fear of the unnatural could shed further light on the persistence of rejection of science.

In the case of rejection of evolution, more targeted measures of religious beliefs might provide further predictive power. Measures of biblical literalism and religious 
fundamentalism might prove more useful in explaining rejection of evolution than the broad measure of religiosity used in Studies 5 and 6 (Rutjens et al., 2017; Zigerell, 2012).

\subsubsection{Beyond individual differences}

The later studies in this thesis focusing on RWA and SDO are essentially grounded in an individual differences approach. That is, they focus on stable, individual traits as a possible root cause of rejection of science. I argue RWA and SDO — based on personality, values and socialisation (as per the DPM; Duckitt \& Sibley, 2016)—underpin known predictors of rejection of science, such as political and religious ideologies and conspiracy thinking. However, ideology and conspiracy thinking are just two of six broad themes outlined by Hornsey and Fielding (2017) in their transtheoretical Attitudes Roots model. The authors also highlight other potential drivers of rejection of science tied to personal and social identities, vested interests and a possible role of fears and phobias.

In the case of personal identity, Hornsey and Fielding (2017; following Katz, 1960) suggest that people some reject scientific findings simply to signal the kind of person they are:

to the extent that people see themselves as "individual", "non-conformist"” or "non-gullible", then defining oneself against scientific consensus can be a way of communicating these qualities. So for someone keen to self-define as a contrarian, climate change scepticism (for example) might be a short-hand way of communicating that identity. (p. 465)

Hornsey, Harris and Fielding's (2018b) international survey of vaccine attitudes offers support for this claim, reporting that anti-vaccination attitudes correlate with scores on a 'reactance' scale (including items such as 'I find contradicting others stimulating'; Hong \& Page, 1989).

In the case of social identities, rejection of science may be attributed not to an individual's personal characteristics but to those of the groups that individual identifies with (and the strength of that identification). This idea is based in the Social Identity Theory (Tajfel \& Turner, 1979), which can be thought of as a theoretical lens through which to consider rejection of science (Fielding \& Hornsey, 2016). This approach recognises that people categorise themselves as part of groups and, to a certain extent, assimilate the norms, values, and beliefs of those groups. For example, people who identify as a conservatives or Christians may adopt the views of their group regarding certain scientific issues. Kahan 
(2012a) makes a similar argument in his outline of the Cultural Cognition Thesis; rejection of science on some issues can be tied to peoples' affinity with certain cultural groups (although see van der Linden, 2015a, for a critique). Social identity theory also highlights the contextdependent nature of identity. Social identities and their associated norms become more or less salient depending on the context (reviewed in Stets \& Burke, 2000).

There is a small but growing body of research applying Social Identity Theory to rejection of science (Nauroth, Gollwitzer, Bender, \& Rothmund, 2015; Unsworth \& Fielding, 2014). For example, Prot (2015) reports that increasing the saliency of videogame users' 'gamer' identity increases their scepticism of research indicating that videogames cause aggressive behaviour. There is also some evidence that climate sceptics, as a group, share a distinct social identity built around rejection of climate science (Bliuc et al., 2015). This is likely also the case for people who consider themselves part of 'vaccine hesitant communities' (Attwell \& Smith, 2017). Where rejection of science is a key aspect of a group's beliefs, more information is unlikely shift those beliefs at the individual level.

However, making certain identities less salient, or emphasising superordinate social identities (e.g. 'Americans' rather than 'conservatives') can aid in dampening the counter-scientific attitudes associated with some groups (reviewed in Fielding \& Hornsey, 2016).

Social identity research should be considered complimentary to, rather than competing with, individual difference approaches to understanding why some people don't align their beliefs with best available evidence. Bliuc et al.'s (2015) investigation of the climate sceptic social identity offers an example of this, merging both approaches. The authors propose that individual political and moral beliefs feed into a 'group consciousness' which in turn predicts specific beliefs and intended behaviours regarding climate change.

In the case of vested interests, the Hornsey and Fielding (2017) note that some individuals may be motivated to reject scientific findings which would create a high personal cost. In the case of climate science this might include people who have a large carbon footprint or work in carbon-polluting industries. For these individuals, accepting the scientific claim that a reduction in carbon emissions would limit global warming — and acting accordingly — could incur unwanted financial or lifestyle costs. In support of this, Tranter and Booth (2015) report that, at a country level, higher $\mathrm{CO}_{2}$ emissions are positively corelated with climate scepticism. Hornsey et al. (2018a) note that per captia $\mathrm{CO}_{2}$ emissions can be seen as representing vested intersts in terms of the individual-level sacrifices citzens must make to trasition to a low-carbon lifestyle. However, they also acknowledge that high 
emissions also represent a corporate vested interest in terms of the fossil fuel industry's investment in a country.

A fulsome consideration of rejection of science should also take into account the role of 'top down' influences such as industry-funded think tanks, lobbyists and political elites (Goertzel, 2018; Jacquet, Dietrich, \& Jost, 2014). Furthermore, in this new era of social media we must be cognizant of deliberate disinformation campaigns undertaken by nationstates seeking sow discord by stirring up scientific controversy (W. L. Bennett \& Livingston, 2018; Lewandowsky, Ecker, et al., 2017). Hornsey et al. (2018a) summarise the situation: ...ideological barriers to accepting science do not just emerge 'bottom-up', in the sense of individuals spontaneously critiquing scientific consensus through the lens of their worldviews. Rather, ideological barriers to accepting science can also be cultivated and nourished 'top-down' by influential individuals and organizations who have a vested interest in communicating the notion that the science is flawed or inconclusive. (p. 615)

The role of vested interests, such as fossil fuel companies, in fostering rejection of climate science has been well documented (Dunlap \& McCright, 2011; Oreskes \& Conway, 2010). Researchers have also highlighted instances where vested interests have sought to increase rejection of science regarding vaccination. For example, Broniatowski et al. (2018) documents the role of Russian twitter bots in disseminating anti-vaccine content, and Kata (2010, 2012) cites numerous instances of alternative medicine retailer websites presenting vaccine misinformation while promoting their own dubious products. While a focus on individuals can offer insight into the persisitence of rejection of science and peoples' susceptibility to misinformation, we cannot ignore the wider context in which this occurs (Lewandowsky, Ecker, et al., 2017).

Hornsey and Fielding (2017) also acknowledge a potential role of fears and phobias as an attitude root rejection of science, offering the example of needle phobia and antivaccine attitudes. They theorise that individuals who suffer a fear of needles may be motivated to accept claims that vaccines are dangerous to justify a refusal to vaccinate. Hornsey, Harris and Fielding (2018b) offer some preliminary evidence that this is the case, reporting that antivaccine attitudes correlate with disgust towards needles and blood in an international survey.

Beyond social-psychological explanations for rejection of science, we should also consider the role of the environment and personal experience in informing personal beliefs about scientific issues. For example, people are more likely to agree climate change is 
occurring on warmer days (Li, Johnson, \& Zaval, 2011; Zaval, Keenan, Johnson, \& Weber, 2014). People who have experienced extreme weather events are more likely to believe human-caused climate change is occurring (Myers, Maibach, Roser-Renouf, Akerlof, \& Leiserowitz, 2013; A. Spence, Poortinga, Butler, \& Pidgeon, 2011). Mothers who have had personal experiences related to human papillomavirus or consequent cervical cancer are more likely to accept the best available evidence and vaccinate their daughters against the virus (Cooper Robbins, Bernard, McCaffery, Brotherton, \& Skinner, 2010). These studies indicate that events outside the control of individuals or groups can also influence our beliefs about important scientific issues.

To sum up, individual differences can go some way in explaining why people argue about science (as shown in this thesis), but they are not the be-all-end-all; a more holistic examination of the phenomenon must also consider the role of identity needs, group dynamics, vested personal and commercial interests, and environmental factors.

\subsubsection{Jumping the attitude-behaviour gap}

Addressing rejection of science is important as misinformed beliefs about scientific issues can lead people to act in ways that are harmful to themselves, others and environment. Belief in the reality of human caused climate change predicts behavioural intention relating personal action (e.g. purchasing fuel efficient cars, reducing air travel), as well as support for government policies such as carbon regulations (Gifford, 2011, 2013). Vaccination safety beliefs predict actual vaccination choices; parents who express vaccine hesitancy are subsequently more likely to refuse to vaccinate their children, placing children at greater risk of infectious diseases (Gilkey et al., 2016; Shapiro et al., 2018). People who believe GM food is dangerous are less accepting of GM foods in policy and consumer contexts, potentially hampering the development of a sustainable global food system (Lucht, 2015; Paarlberg, 2015). Concerns about the safety of fluoride can be assumed to lead to avoidance of fluoridated water, toothpaste and dental treatments, increasing the risk of dental caries, although behavioural research is limited (Armfield \& Akers, 2010; Chi, 2017). In sum, previous research indicates that the beliefs examined in this thesis can lead to behaviours that, from an environmental and public health perspective, are undesirable.

However, accepting the scientific consensus on these issues does not automatically lead to expected behaviours. Within models of behaviour such as the Theory of Planned Behaviour (Ajzen, 1991), personal beliefs and attitudes are just one part of the puzzle when it 
comes to explaining why people do the things they do; subjective norms and perceived behavioural control also have a bearing on intentions to act. ${ }^{29}$ Thus, in pursuit of the goal of getting people to act in the best interest of themselves, others and the planet, we should not focus only on self-reported agreement with the best available evidence. Individuals' beliefs about others and their perceptions of the feasibility of behaviours also have a bearing their final actions.

\subsubsection{Changing beliefs}

The research presented in the second half of this thesis paints a relatively consistent picture of the psychological predictors of rejection of science but falls short of providing evidence that these antecedents can be leveraged to shift people's beliefs. These later studies should inform the development of strategies to overcome barriers to acceptance of science, for example aligning communications to fit with underlying ideological motivations (Hornsey $\&$ Fielding, 2017). However, these approaches must be tested experimentally to assess (and hopefully confirm) their efficacy. For example, do messages which emphasise the hierarchical elements of the science sector increase trust in science among high SDO individuals (as suggested in Section 6.5.4)? And, to link this to the wider arc of the entire thesis, would this increase in trust make high SDOs more receptive to the scientific consensus on publicly debated issues?

\subsection{CLOSING COMMENT}

In the face of the 'post-truth' claims we need not throw up our hands. Through research we can answer questions about the 'why' of rejection of science - why people hold beliefs that go against the best available evidence and scientific consensus. Firstly, just knowing that there is a scientific consensus could be a factor. Telling people that most scientists agree appears to have some impact on personal beliefs about contested science, though one's perception of scientific agreement may also be skewed by their own beliefs.

Deeper beliefs about society are also entangled with rejection (and acceptance) of science. For example, I've shown that conservative political leanings are linked with an interest in climate and vaccine sceptical content on social media. The final studies in this thesis reveal a more complex picture of the ideological underpinnings of rejection of science.

\footnotetext{
${ }^{29}$ Technically the Theory of Planned Behaviour draws on evaluative (e.g. vaccination is good/bad) attitudes towards an attitude object, not specific beliefs (e.g. vaccines are toxic). However, as Azjen and Cotes (2008) note, beliefs are considered to be the prevailing determinants of a person's attitudes (see p. 290).
} 
Two broad sets of beliefs regarding authority and dominance are linked to rejection of science across several very different topics and the reasons for this vary across issues. However, a general distrust of science and scientists emerges a consistent link; people who embrace authoritarian and hierarchical values are less likely to trust scientists and, in turn, are more likely disagree with the findings of scientists. This final finding offers new theoretical and practical insights which can ultimately inform efforts to change people's beliefs about socially relevant scientific issues. 



\section{References}

Adams, D. (2013, June). "Hamilton is going to regret this": Council votes to stop fluoride. Waikato Times. Retrieved from http://www.stuff.co.nz/waikatotimes/8761302/Hamilton-is-going-to-regret-this-Council-votes-to-stop-fluoride

Adorno, T. W., Frenkle-Brunswik, E., Levinson, D. J., \& Sanford, R. N. (1950). The Authoritarian Personality. New York: Harper \& Brothers.

Ajzen, I. (1991). The theory of planned behavior. Organizational Behavior and Human Decision Processes, 50(2), 179-211. https://doi.org/10.1016/07495978(91)90020-T

Ajzen, I., \& Cote, N. G. (2008). Attitudes and the prediction of behaviour. In W. D. Crano \& R. Prislin (Eds.), Attitudes and attitude change (pp. 289-311). New York, NY: Taylor \& Francis Group.

Aklin, M., \& Urpelainen, J. (2014). Perceptions of scientific dissent undermine public support for environmental policy. Environmental Science and Policy, 38, 173-177. https://doi.org/10.1016/j.envsci.2013.10.006

Allukian, M., Carter-Pokras, O. D., Gooch, B. F., Horowitz, A. M., Iida, H., Jacob, M., ... Rozier, R. G. (2018). Science, politics, and communication: The case of community water fluoridation in the US. Annals of Epidemiology, 28(6), 401410. https://doi.org/10.1016/J.ANNEPIDEM.2017.05.014

Allum, N., Allansdottir, A., Gaskell, G., Hampel, J., Jackson, J., Moldovan, A., ... Stoneman, P. (2017). Religion and the public ethics of stem-cell research: Attitudes in Europe, Canada and the United States. PLoS ONE, 12(4). https://doi.org/10.1371/JOURNAL.PONE.0176274

Allum, N., Sturgis, P., Tabourazi, D., \& Brunton-Smith, I. (2008). Science knowledge and attitudes across cultures: a meta-analysis. Public Understanding of Science, 17(1), 35-54. https://doi.org/10.1177/0963662506070159

Almendrala, A. (2016, August 2). What the science really says about GMOs and food safety. Huffington Post. Retrieved from

http://www.huffingtonpost.com/entry/that-new-gmo-labeling-law-doesnt-alignwith-scientific-consensus-on-gmo-safety_us_57a0ca4ae4b0693164c2c3a6

Altemeyer, B. (1988). Enemies of Freedom: Understanding Right-Wing Authoritarianism. San Francisco: Jossey-Bass.

Altemeyer, B. (1996). The Authoritarian Spectre. Cambridge, MA, US: Harvard University Press.

Altemeyer, B. (1998). The Other "Authoritarian Personality." Advances in Experimental Social Psychology, 30, 47-92. https://doi.org/10.1016/S00652601(08)60382-2

Altemeyer, B. (2004). Highly dominating, highly authoritarian personalities. The Journal of Social Psychology, 144(4), 421-448. 
https://doi.org/10.3200/SOCP.144.4.421-448

Altemeyer, B. (2006). The Authoritarians. Retrieved from https://theauthoritarians.org/Downloads/TheAuthoritarians.pdf

Altemeyer, B., \& Hunsberger, B. (1992). Authoritarianism, religious fundamentalism, quest, and prejudice. International Journal for the Psychology of Religion, 2(2), 113-133. https://doi.org/10.1207/s15327582ijpr0202_5

Arbuckle, J. L. (2014). Amos (Version 23.0) [Computer Program]. Chicago: IBM SPSS.

Armfield, J. M. (2007). When public action undermines public health: a critical examination of antifluoridationist literature. Australia and New Zealand Health Policy, 4(1), 25. https://doi.org/10.1186/1743-8462-4-25

Armfield, J. M., \& Akers, H. F. (2010). Risk perception and water fluoridation support and opposition in Australia. Journal of Public Health Dentistry, 70(1), 58-66. https://doi.org/10.1111/j.1752-7325.2009.00144.x

Asbrock, F., Sibley, C. G., \& Duckitt, J. (2009). Right-wing authoritarianism and social dominance orientation and the dimensions of generalized prejudice: A longitudinal test. European Journal of Personality, 24(4), n/a-n/a. https://doi.org/10.1002/per.746

Attwell, K., \& Smith, D. T. (2017). Parenting as politics: social identity theory and vaccine hesitant communities. International Journal of Health Governance, 22(3), 183-198. https://doi.org/10.1108/IJHG-03-2017-0008

Azevedo, F., Jost, J. T., Rothmund, T., \& Sterling, J. (2019). Neoliberal ideology and the justification of inequality in capitalist societies: Why social and economic dimensions of ideology are intertwined. Journal of Social Issues, 75(1), 49-88. https://doi.org/10.1111/josi.12310

Baldwin, M., \& Lammers, J. (2016). Past-focused environmental comparisons promote proenvironmental outcomes for conservatives. Proceedings of the National Academy of Sciences of the United States of America, 113(52), 1495314957. https://doi.org/10.1073/pnas.1610834113

Barberá, P. (2015). Birds of the same feather tweet together: Bayesian ideal point estimation using Twitter data. Political Analysis, 23(1), 76-91. https://doi.org/10.1093/pan/mpu011

Barberá, P., Jost, J. T., Nagler, J., Tucker, J. A., \& Bonneau, R. (2015). Tweeting from left to right. Psychological Science, 26(10), 1531-1542. https://doi.org/10.1177/0956797615594620

Baron, J., \& Jost, J. T. (2019). False equivalence: Are liberals and conservatives in the United States equally biased? Perspectives on Psychological Science, 14(2), 292-303. https://doi.org/10.1177/1745691618788876

Baron, R. M., \& Kenny, D. A. (1986). The moderator-mediator variable distinction in social psychological research: conceptual, strategic, and statistical considerations. Journal of Personality and Social Psychology, 51(6), 11731182. Retrieved from http://www.ncbi.nlm.nih.gov/pubmed/3806354 
Baumgaertner, B., Carlisle, J. E., \& Justwan, F. (2018). The influence of political ideology and trust on willingness to vaccinate. PLOS ONE, 13(1), e0191728. https://doi.org/10.1371/journal.pone.0191728

Bennett, L. (2019, March 6). Worries over anti-vaxxers: DHBs say diseases like measles are being seen again as infant immunisation declines. New Zealand Herald. Retrieved from https://www.nzherald.co.nz/nz/news/article.cfm?c_id=1\&objectid=12210170

Bennett, W. L., \& Livingston, S. (2018). The disinformation order: Disruptive communication and the decline of democratic institutions. European Journal of Communication, 33(2), 122-139. https://doi.org/10.1177/0267323118760317

Biel, A., \& Nilsson, A. (2005). Religious values and environmental concern: Harmony and detachment. Social Science Quarterly, 86(1), 178-191. https://doi.org/10.1111/j.0038-4941.2005.00297.x

Biological Heritage. (2018). Exploring public acceptance novel technologies wide scale pest control [YouTube video]. Retrieved from www.youtube.com/watch? v=Z1vcHbHEdBE

Björnberg, K. E., Karlsson, M., Gilek, M., \& Hansson, S. O. (2017). Climate and environmental science denial: A review of the scientific literature published in 1990-2015. Journal of Cleaner Production, 167, 229-241. https://doi.org/10.1016/J.JCLEPRO.2017.08.066

Blancke, S., Van Breusegem, F., De Jaeger, G., Braeckman, J., \& Van Montagu, M. (2015). Fatal attraction: The intuitive appeal of GMO opposition. Trends in Plant Science, 20(7), 414-418. https://doi.org/10.1016/J.TPLANTS.2015.03.011

Blank, J. M., \& Shaw, D. (2015). Does partisanship shape attitudes toward science and public policy? The case for ideology and religion. The ANNALS of the American Academy of Political and Social Science, 658(1), 18-35. https://doi.org/10.1177/0002716214554756

Bliuc, A.-M., McGarty, C., Thomas, E. F., Lala, G., Berndsen, M., \& Misajon, R. (2015). Public division about climate change rooted in conflicting sociopolitical identities. Nature Climate Change, 5(3), 226-229. https://doi.org/10.1038/nclimate2507

Bobbio, N. (1996). Left and Right: The Significance of a Political Distinction. Cambridge, UK: Polity Press.

Bolsen, T., \& Druckman, J. N. (2015). Counteracting the politicization of science. Journal of Communication, 65(5), 745-769. https://doi.org/10.1111/jcom.12171

Bolsen, T., \& Druckman, J. N. (2018a). Do partisanship and politicization undermine the impact of a scientific consensus message about climate change? Group Processes \& Intergroup Relations, 21(3), 389-402. https://doi.org/10.1177/1368430217737855

Bolsen, T., \& Druckman, J. N. (2018b). Validating conspiracy beliefs and effectively communicating scientific consensus. Weather, Climate, and Society, 10(3), 
453-458. https://doi.org/10.1175/WCAS-D-17-0096.1

Bolsen, T., Leeper, T. J., \& Shapiro, M. A. (2014). Doing what others do: Norms, science, and collective action on global warming. American Politics Research, 42(1), 65-89. https://doi.org/10.1177/1532673X13484173

Bord, R. J., O'Connor, R. E., \& Fisher, A. (2000). In what sense does the public need to understand global climate change? Public Understand of Science, 9(3), 205218. Retrieved from https://journals.sagepub.com/doi/pdf/10.1088/0963$6625 / 9 / 3 / 301$

Boutyline, A., \& Willer, R. (2017). The social structure of political echo chambers: Variation in ideological homophily in online networks. Political Psychology, 38(3), 551-569. https://doi.org/10.1111/pops.12337

Boyd, K. (2019). Epistemically pernicious groups and the groupstrapping problem. Social Epistemology, 33(1), 61-73. https://doi.org/10.1080/02691728.2018.1551436

Brewer, P. R., \& Ley, B. L. (2013). Whose science do you believe? Explaining trust in sources of scientific information about the environment. Science Communication, 35(1), 115-137. https://doi.org/10.1177/1075547012441691

Brewer, P. R., \& McKnight, J. (2017). "A statistically representative climate change debate": Satirical television news, scientific consensus, and public perceptions of global warming. Atlantic Journal of Communication, 25(3), 166-180. https://doi.org/10.1080/15456870.2017.1324453

Broadbent, J., Wills, R., McMillan, J., Drummond, B., \& Whyman, R. (2015). Evaluation of evidence behind some recent claims against community water fluoridation in New Zealand. Journal of the Royal Society of New Zealand, 45(3), 161-178. https://doi.org/10.1080/03036758.2015.1056193

Broniatowski, D. A., Jamison, A. M., Qi, S., AlKulaib, L., Chen, T., Benton, A., ... Dredze, M. (2018). Weaponized health communication: Twitter bots and russian trolls amplify the vaccine debate. American Journal of Public Health, 108(10), 1378-1384. https://doi.org/10.2105/AJPH.2018.304567

Broomell, S. B., \& Kane, P. B. (2017). Public perception and communication of scientific uncertainty. Journal of Experimental Psychology: General, 146(2), 286-304. https://doi.org/10.1037/xge0000260

Brossard, D., \& Nisbet, M. C. (2006). Deference to scientific authority among a low information public: Understanding U.S. opinion on agricultural biotechnology. International Journal of Public Opinion Research, 19(1), 24-52. https://doi.org/10.1093/ijpor/ed1003

Brotherton, R., French, C. C., \& Pickering, A. D. (2013). Measuring belief in conspiracy theories: The Generic Conspiracist Beliefs Scale. Frontiers in Psychology, 4, 279. https://doi.org/10.3389/fpsyg.2013.00279

Browne, M. (2018). Epistemic divides and ontological confusions: The psychology of vaccine scepticism. Human Vaccines \& Immunotherapeutics, 14(10), 25402542. https://doi.org/10.1080/21645515.2018.1480244 
Browne, M., Thomson, P., Rockloff, M. J., \& Pennycook, G. (2015). Going against the herd: Psychological and cultural factors underlying the "vaccination confidence gap. PLOS ONE, 10(9), e0132562. https://doi.org/10.1371/journal.pone.0132562

Bruder, M., Haffke, P., Neave, N., Nouripanah, N., \& Imhoff, R. (2013). Measuring individual differences in generic beliefs in conspiracy theories across cultures: Conspiracy Mentality Questionnaire. Frontiers in Psychology, 4, 225. https://doi.org/10.3389/fpsyg.2013.00225

Buckland, C. R. (2014). Examining the underlying complexity of free market beliefs (Western University, Canada). Retrieved from https://ir.lib.uwo.ca/cgi/viewcontent.cgi?article=1011\&context=psychK_uht

Burstein, P. (2003). The Impact of Public Opinion on Public Policy: A Review and an Agenda. Political Research Quarterly, 56(1), 29-40. https://doi.org/10.1177/106591290305600103

Campbell, T. H., \& Kay, A. C. (2014). Solution aversion: On the relation between ideology and motivated disbelief. Journal of Personality and Social Psychology, 107(5), 809-824. https://doi.org/10.1037/a0037963

Cantal, C. (2015). "Direct threats require decisive action": An investigation on how levels of political conservatism change in the face of economic, natural and social threats (Victoria University of Wellington, New Zealand). Retrieved from http://researcharchive.vuw.ac.nz/handle/10063/4950

Cantal, C., Milfont, T. L., Wilson, M. S., \& Gouveia, V. V. (2015). Differential effects of right-wing authoritarianism and social dominance orientation on dimensions of generalized prejudice in Brazil. European Journal of Personality, 29(1), 17-27. https://doi.org/10.1002/per.1978

Carbon Brief. (2016). Mapped: The climate change conversation on Twitter |Carbon Brief. Retrieved June 25, 2019, from https://www.carbonbrief.org/mapped-theclimate-change-conversation-on-twitter

Carl, N., \& Cofnas, N. (2016). Scientific literacy, optimism about science and conservatism. Personality and Individual Differences, 94, 299-302. https://doi.org/10.1016/J.PAID.2016.01.046

Chaiken, S., Liberman, A., \& Eagly, A. H. (1989). Heuristic and systematic information processing within and beyond the persuasion context. In J. S. Uleman \& J. A. Bargh (Eds.), Unintended Thought (pp. 212-252). New York: Guilford.

Chance, Z., \& Norton, M. I. (2015). The what and why of self-deception. Current Opinion in Psychology, 6, 104-107. https://doi.org/10.1016/J.COPSYC.2015.07.008

Chen, E. E., \& Wojcik, S. P. (2016). A practical guide to big data research in psychology. Psychological Methods, 21(4), 458-474. https://doi.org/10.1037/met0000111

Chetty, K., Devadas, V., \& Fleming, J. (2015). The framing of climate change in 
New Zealand newspapers from June 2009 to June 2010. Journal of the Royal Society of New Zealand, 45(1), 1-20.

https://doi.org/10.1080/03036758.2014.996234

Chi, D. (2017). Parent refusal of topical fluoride for their children: Clinical strategies and future research priorities to improve evidence-based pediatric dental practice. Dental Clinics of North America, 61(3), 607-617. https://doi.org/10.1016/J.CDEN.2017.03.002

Chikazhe, T. L. (2015). New Zealand public attitudes towards genetically modified food (Lincoln University, New Zealand). Retrieved from https://researcharchive.lincoln.ac.nz/handle/10182/6825

Chinn, S., Lane, D. S., \& Hart, P. S. (2018). In consensus we trust? Persuasive effects of scientific consensus communication. Public Understanding of Science, 27(7), 807-823. https://doi.org/10.1177/0963662518791094

Clayton, S., Devine-Wright, P., Stern, P. C., Whitmarsh, L., Carrico, A., Steg, L., ... Bonnes, M. (2015). Psychological research and global climate change. Nature Climate Change, 5(7), 640-646. https://doi.org/10.1038/nclimate2622

Cloud, D. (2016). Communicating climate change to religious and conservative audiences: The case of Katharine Hayhoe and Andrew Farley. Reflections, 16(1), 57-74. Retrieved from https://reflectionsjournal.net/wpcontent/uploads/CopyrightUpdates/Vol16N1/16.1-Communicating-ClimateChange-to-Religious-and-Conservative-Audiences-Cloud.pdf

Cohen, J. (1988). Statistical Power Analysis for the Behavioural Sciences (2nd ed.). Hillsdale, NJ: Lawrence Erlbaum.

Cohen, P., Cohen, J., Aiken, L. S., \& West, S. G. (1999). The problem of units and the circumstance for POMP. Multivariate Behavioral Research, 34(3), 315-346. https://doi.org/10.1207/S15327906MBR3403_2

Cohen, P. N. (2018). The widening political divide over science. https://doi.org/10.31235/osf.io/u95aw

Converse, P. E. (2006). The nature of belief systems in mass publics (1964). Critical Review, 18(1), 1-74. https://doi.org/10.1080/08913810608443650

Cook, J. (2017). Response by Cook to "Beyond Counting Climate Consensus." Environmental Communication, 11(6), 733-735. https://doi.org/10.1080/17524032.2017.1377095

Cook, J., \& Lewandowsky, S. (2016). Rational irrationality: Modeling climate change belief polarization using Bayesian networks. Topics in Cognitive Science, 8(1), 160-179. https://doi.org/10.1111/tops.12186

Cook, J., Lewandowsky, S., \& Ecker, U. K. H. (2017). Neutralizing misinformation through inoculation: Exposing misleading argumentation techniques reduces their influence. PLOS ONE, 12(5), e0175799. https://doi.org/10.1371/journal.pone.0175799

Cook, J., Oreskes, N., Doran, P. T., Anderegg, W. R. L., Verheggen, B., Maibach, E. W., ... Rice, K. (2016). Consensus on consensus: a synthesis of consensus 
estimates on human-caused global warming. Environmental Research Letters, 11(4), 048002. https://doi.org/10.1088/1748-9326/11/4/048002

Cooper Robbins, S. C., Bernard, D., McCaffery, K., Brotherton, J. M. L., \& Skinner, S. R. (2010). "I just signed": Factors influencing decision-making for schoolbased HPV vaccination of adolescent girls. Health Psychology, 29(6), 618-625. https://doi.org/10.1037/a0021449

Corner, A., Whitmarsh, L., \& Xenias, D. (2012). Uncertainty, scepticism and attitudes towards climate change: biased assimilation and attitude polarisation. Climatic Change, 114(3-4), 463-478. https://doi.org/10.1007/s10584-0120424-6

Costa-Font, M., Gil, J. M., \& Traill, W. B. (2008). Consumer acceptance, valuation of and attitudes towards genetically modified food: Review and implications for food policy. Food Policy, 33(2), 99-111. https://doi.org/10.1016/j.foodpol.2007.07.002

Crawford, J. T., Brady, J. L., Pilanski, J. M., Erny, H., \& Erny, H. (2013). Differential effects of right-wing authoritarianism and social dominance orientation on political candidate support: The moderating role of message framing. Journal of Social and Political Psychology, 1(1), 5-28. https://doi.org/10.5964/jspp.v1i1.170

Dallago, F., Cima, R., Roccato, M., Ricolfi, L., \& Mirisola, A. (2008). The correlation between right-wing authoritarianism and social dominance orientation: The moderating effects of political and religious identity. Basic and Applied Social Psychology, 30(4), 362-368.

https://doi.org/10.1080/01973530802502333

David, M., \& Poynton, M. (2015). Review of the benefits and costs of water fluoridation in New Zealand. Retrieved from https://www.health.govt.nz/publication/review-benefits-and-costs-waterfluoridation-new-zealand

de Boer, J., Schösler, H., \& Boersema, J. J. (2013). Climate change and meat eating: An inconvenient couple? Journal of Environmental Psychology, 33, 1-8. https://doi.org/10.1016/J.JENVP.2012.09.001

De Pryck, K., \& Gemenne, F. (2017). The Denier-in-Chief: Climate change, science and the election of Donald J. Trump. Law and Critique, 28(2), 119-126. https://doi.org/10.1007/s10978-017-9207-6

Dębski, M., \& Niemczak, K. (2014). The image of Germany in Poland and its impact on development of Poles travelling tourism to Germany. Journal of Intercultural Management, 6(1), 67-79. https://doi.org/10.2478/joim-2014-0005

Delaney, B., Goodman, R. E., \& Ladics, G. S. (2018). Food and feed safety of genetically engineered food crops. Toxicological Sciences, 162(2), 361-371. https://doi.org/10.1093/toxsci/kfx249

Deryugina, T., \& Shurchkov, O. (2016). The effect of information provision on public consensus about climate change. PLoS ONE, 11(4). https://doi.org/10.1371/journal.pone.0151469 
Dietz, T. (2013). Bringing values and deliberation to science communication. Proceedings of the National Academy of Sciences of the United States of America, 110(Supplement 3), 14081-14087. https://doi.org/10.1073/pnas.1212740110

Dietz, T., Dan, A., \& Shwom, R. (2007). Support for climate change policy: Social psychological and social structural influences. Rural Sociology, 72(2), 185-214. https://doi.org/10.1526/003601107781170026

Ding, D., Maibach, E. W., Zhao, X., Roser-Renouf, C., \& Leiserowitz, A. (2011). Support for climate policy and societal action are linked to perceptions about scientific agreement. Nature Climate Change, 1(9), 462-466. https://doi.org/http://dx.doi.org/10.1038/nclimate1295

Diresta, R., \& Lotan, G. (2016, August). Anti-vaxxers are using twitter to manipulate a vaccine bill. Wired. Retrieved from https://www.wired.com/2015/06/antivaxxers-influencing-legislation/

Ditto, P. H., Clark, C. J., Liu, B. S., Wojcik, S. P., Chen, E. E., Grady, R. H., ... Zinger, J. F. (2019). Partisan bias and its discontents. Perspectives on Psychological Science, 14(2), 304-316. https://doi.org/10.1177/1745691618817753

Ditto, P. H., Liu, B. S., Clark, C. J., Wojcik, S. P., Chen, E. E., Grady, R. H., ... Zinger, J. F. (2019). At least bias is bipartisan: A meta-analytic comparison of partisan bias in liberals and conservatives. Perspectives on Psychological Science, 14(2), 273-291. https://doi.org/10.1177/1745691617746796

Dixon, G. N. (2016). Applying the Gateway Belief Model to genetically modified food perceptions: New insights and additional questions. Journal of Communication, 66(6), 888-908. https://doi.org/10.1111/jcom.12260

Dixon, G. N., \& Clarke, C. E. (2013). Heightening uncertainty around certain science. Science Communication, 35(3), 358-382. https://doi.org/10.1177/1075547012458290

Dixon, G. N., Hmielowski, J., \& Ma, Y. (2017). Improving climate change acceptance among U.S. conservatives through value-based message targeting. Science Communication, 39(4), 520-534. https://doi.org/10.1177/1075547017715473

Dixon, G. N., McKeever, B. W., Holton, A. E., Clarke, C., \& Eosco, G. (2015). The Power of a picture: Overcoming scientific misinformation by communicating weight-of-evidence information with visual exemplars. Journal of Communication, 65(4), 639-659. https://doi.org/10.1111/jcom.12159

Dobzhansky, T. (1973). Nothing in biology makes sense except in the light of evolution. The American Biology Teacher, 35(3), 125-129. Retrieved from http://www.jstor.org/stable/4444260

Domingo, J. L. (2016). Safety assessment of GM plants: An updated review of the scientific literature. Food and Chemical Toxicology, 95, 12-18. https://doi.org/10.1016/J.FCT.2016.06.013 
Douglas, M., \& Wildavsky, A. B. (1982). Risk and culture: an essay on the selection of technical and environmental dangers. Berkley: University of California Press.

Dredze, M., Broniatowski, D. A., Smith, M. C., \& Hilyard, K. M. (2016). Understanding vaccine refusal: Why we need social media now. American Journal of Preventive Medicine, 50(4), 550-552. https://doi.org/10.1016/j.amepre.2015.10.002

Druckman, J. N. (2017). The crisis of politicization within and beyond science. Nature Human Behaviour, 1(9), 615-617. https://doi.org/10.1038/s41562-0170183-5

Druckman, J. N., \& McGrath, M. C. (2019). The evidence for motivated reasoning in climate change preference formation. Nature Climate Change, 9(2), 111-119. https://doi.org/10.1038/s41558-018-0360-1

Drummond, C., \& Fischhoff, B. (2017). Individuals with greater science literacy and education have more polarized beliefs on controversial science topics. Proceedings of the National Academy of Sciences of the United States of America, 114(36), 9587-9592. https://doi.org/10.1073/pnas.1704882114

Duckitt, J., Bizumic, B., Krauss, S. W., \& Heled, E. (2010). A tripartite approach to right-wing authoritarianism: The Authoritarianism-Conservatism-Traditionalism model. Political Psychology, 31(5), 685-715. https://doi.org/10.1111/j.14679221.2010.00781.x

Duckitt, J., \& Fisher, K. (2003). The impact of social threat on worldview and ideological attitudes. Political Psychology, 24(1), 199-222. https://doi.org/10.1111/0162-895X.00322

Duckitt, J., \& Sibley, C. G. (2007). Right wing authoritarianism, social dominance orientation and the dimensions of generalized prejudice. European Journal of Personality, 21(2), 113-130. https://doi.org/10.1002/per.614

Duckitt, J., \& Sibley, C. G. (2009). A dual-process motivational model of ideology, politics, and prejudice. Psychological Inquiry, 20(2-3), 98-109. https://doi.org/10.1080/10478400903028540

Duckitt, J., \& Sibley, C. G. (2016). The dual process motivational model of ideology and prejudice. In C. G. Sibley \& F. K. Barlow (Eds.), The Cambridge Handbook of the Psychology of Prejudice (pp. 188-221). https://doi.org/10.1017/9781316161579.009

Dunlap, R. E., \& McCright, A. M. (2011). Organized climate change denial. In D. Dryzek, John S. Norgaard, Richard B. Schlosberg (Ed.), The Oxford Handbook of Climate Change and Society. https://doi.org/10.1093/oxfordhb/9780199566600.003.0010

Dunwoody, S., \& Kohl, P. A. (2017). Using weight-of-experts messaging to communicate accurately about contested science. Science Communication, 39(3), 338-357. https://doi.org/10.1177/1075547017707765

Duriez, B., Van Hiel, A., \& Kossowska, M. (2005). Authoritarianism and social 
dominance in Western and Eastern Europe: The importance of the sociopolitical context and of political interest and involvement. Political Psychology, 26(2), 299-320. https://doi.org/10.1111/j.1467-9221.2005.00419.x

Eady, G., Nagler, J., Guess, A., Zilinsky, J., \& Tucker, J. A. (2019). How many people live in political bubbles on social media? Evidence from linked survey and twitter data. SAGE Open, $9(1)$.

https://doi.org/https://doi.org/10.1177/2158244019832705

Ecklund, E. H., \& Scheitle, C. P. (2018). Religious people are climate change deniers. In E. H. Ecklund \& C. P. Scheitle (Eds.), Religion vs. Science: What Religious People Really Think. https://doi.org/10.1093/oso/9780190650629.003.0006

Ecklund, E. H., Scheitle, C. P., Peifer, J., \& Bolger, D. (2017). Examining links between religion, evolution views, and climate change skepticism. Environment and Behavior, 49(9), 985-1006. https://doi.org/10.1177/0013916516674246

Edwards, S. (2017). Research into genetically modified organisms in new zealand: An examination of a sociotechnical controversy. Case Studies in the Environment, 1(1), 1.14-8. https://doi.org/10.1525/cse.2017.000547

Elder, L., Greene, S., \& Lizotte, M. K. (2018). The gender gap on public opinion towards genetically modified foods. The Social Science Journal, 55(4), 500 509. https://doi.org/10.1016/J.SOSCIJ.2018.02.015

Elliott, K. C., \& Resnik, D. B. (2014). Science, policy, and the transparency of values. Environmental Health Perspectives, 122(7), 647-650. https://doi.org/10.1289/ehp.1408107

Environmental Protection Agency. (2017). Annual Report. Retrieved from https://epa.govt.nz/assets/RecordsAPI/70b79333df/EPA-Annual-Report2017.pdf

Evans, G., \& Durant, J. (1995). The relationship between knowledge and attitudes in the public understanding of science in Britain. Public Understanding of Science, 4(1), 57-74. https://doi.org/10.1088/0963-6625/4/1/004

Farias, M., Newheiser, A.-K., Kahane, G., \& de Toledo, Z. (2013). Scientific faith: Belief in science increases in the face of stress and existential anxiety. Journal of Experimental Social Psychology, 49(6), 1210-1213. https://doi.org/10.1016/j.jesp.2013.05.008

Federico, C. M., Weber, C. R., Ergun, D., \& Hunt, C. (2013). Mapping the connections between politics and morality: The multiple sociopolitical orientations involved in moral intuition. Political Psychology, 34(4), 589-610. https://doi.org/10.1111/pops.12006

Feldman, L., Maibach, E. W., Roser-Renouf, C., \& Leiserowitz, A. (2012). Climate on cable. The International Journal of Press/Politics, 17(1), 3-31. https://doi.org/10.1177/1940161211425410

Feldman, L., Myers, T. A., Hmielowski, J. D., \& Leiserowitz, A. (2014). The mutual reinforcement of media selectivity and effects: Testing the reinforcing spirals 
framework in the context of global warming. Journal of Communication, 64(4), 590-611. https://doi.org/10.1111/jcom.12108

Feldman, S., \& Johnston, C. (2014). Understanding the Determinants of Political Ideology: Implications of Structural Complexity. Political Psychology, 35(3), 337-358. https://doi.org/10.1111/pops.12055

Félonneau, M., \& Becker, M. (2008). Pro-environmental attitudes and behavior: Revealing perceived social desirability. Revue Internationale de Psychologie Sociale, 21(4), 25-53.

Ferguson, C. J., \& Wang, J. C. K. (2019). Aggressive Video Games are Not a Risk Factor for Future Aggression in Youth: A Longitudinal Study. Journal of Youth and Adolescence , 48(8), 1439-1451. https://doi.org/10.1007/s10964-019-010690

Fernbach, P. M., Light, N., Scott, S. E., Inbar, Y., \& Rozin, P. (2019). Extreme opponents of genetically modified foods know the least but think they know the most. Nature Human Behaviour, 3(3), 251-256. https://doi.org/10.1038/s41562018-0520-3

Festinger, L. (1957). A theory of cognitive dissonance. Stanford, CA: Stanford University Press.

Field, A. (2009). Discovering statistics using SPSS (3rd ed.). London: SAGE Publications.

Fielding, K. S., \& Hornsey, M. J. (2016). A social identity analysis of climate change and environmental attitudes and behaviors: Insights and opportunities. Frontiers in Psychology, 7, 121. https://doi.org/10.3389/fpsyg.2016.00121

Fields, J. (2015). GMO vs. Organic Food: Top 100 Influencers and Brands.

Retrieved June 25, 2019, from https://onalytica.com/blog/posts/gmo-vs-organicfood-top-100-influencers-and-brands/

Fine, P., Eames, K., \& Heymann, D. L. (2011). Herd Immunity: A Rough Guide. Clinical Infectious Diseases, 52(7), 911-916. https://doi.org/10.1093/cid/cir007

Finkel, S. E. (1995). Causal analysis with panel data. Thousand Oaks, CA: Sage Publications.

Fiske, S. T., \& Dupree, C. (2014). Gaining trust as well as respect in communicating to motivated audiences about science topics. Proceedings of the National Academy of Sciences of the United States of America, 111 Suppl 4(Supplement 4), 13593-13597. https://doi.org/10.1073/pnas.1317505111

Freedman, D. H. (2013). Are engineered foods evil? Scientific American, 309(3), 8085. https://doi.org/10.1038/scientificamerican0913-80

Fritsche, S., Poovaiah, C., MacRae, E., \& Thorlby, G. (2018). A New Zealand Perspective on the Application and Regulation of Gene Editing. Frontiers in Plant Science, 9, 1323. https://doi.org/10.3389/fpls.2018.01323

Funk, C., \& Rainie, L. (2015). Politics and Science: What Americans Think. Retrieved from https://www.pewresearch.org/science/2015/07/01/americans- 
politics-and-science-issues/

Galloway, J. (2016). NZ will have to allow GM food to keep up with other countries. NZ Farmer. Retrieved from http://www.stuff.co.nz/business/farming/78586826/nz-will-have-to-allow-gmfood-to-keep-up-with-other-countries

Gana, K., \& Broc, G. (2018). Structural Equation Modeling with lavaan. https://doi.org/10.1002/9781119579038

Gauchat, G. (2012). Politicization of science in the public sphere. American Sociological Review, 77(2), 167-187. https://doi.org/10.1177/0003122412438225

GE Free NZ. (2016). Proposal to stop labelling of GM food would deny consumers basic right to know. Retrieved August 23, 2017, from http://press.gefree.org.nz/press/20160823.htm

George, D., \& Mallery, P. (2003). SPSS for Windows step by step : a simple guide and reference. Boston, MA: Allyn and Bacon.

Gewin, V. (2017). Communication: Post-truth predicaments. Nature, 541(7637), 425-427. https://doi.org/10.1038/nj7637-425a

Gifford, R. (2011). The dragons of inaction: Psychological barriers that limit climate change mitigation and adaptation. American Psychologist, 66(4), 290-302. https://doi.org/10.1037/a0023566

Gifford, R. (2013). Dragons, mules, and honeybees: Barriers, carriers, and unwitting enablers of climate change action. Bulletin of the Atomic Scientists, 69(4), 4148. https://doi.org/10.1177/0096340213493258

Gilkey, M. B., Reiter, P. L., Magnus, B. E., McRee, A.-L., Dempsey, A. F., \& Brewer, N. T. (2016). Validation of the Vaccination Confidence Scale: A brief measure to identify parents at risk for refusing adolescent vaccines. Academic Pediatrics, 16(1), 42-49. https://doi.org/10.1016/J.ACAP.2015.06.007

Gluckman, P. (2013). Interpreting science-implications for public understanding, advocacy and policy formation. Retrieved from https://www.pmcsa.org.nz/wpcontent/uploads/Interpreting-Science-April-2013.pdf

Goertzel, T. (2010). Conspiracy theories in science. EMBO Reports, 11(7), 493-499. https://doi.org/10.1038/embor.2010.84

Goertzel, T. (2018). The conspiracy theory pyramid scheme. In J. E. Uscinski (Ed.), Conspiracy Theories and the People Who Believe Them (pp. 226-242). https://doi.org/10.1093/oso/9780190844073.003.0015

Golbeck, J., \& Hansen, D. (2014). A method for computing political preference among Twitter followers. Social Networks, 36, 177-184. https://doi.org/10.1016/J.SOCNET.2013.07.004

Golec de Zavala, A., \& Cichocka, A. (2012). Collective narcissism and antiSemitism in Poland. Group Processes \& Intergroup Relations, 15(2), 213-229. https://doi.org/10.1177/1368430211420891 
Grabenstein, J. D. (2013). What the World's religions teach, applied to vaccines and immune globulins. Vaccine, 31(16), 2011-2023.

https://doi.org/10.1016/J.VACCINE.2013.02.026

Grace, G. (2010). Reflection on the university and the academic as "critic and conscience of society." New Zealand Journal of Educational Studies, 45(2), 8992.

Graham, J., Nosek, B. A., Haidt, J., Iyer, R., Koleva, S., \& Ditto, P. H. (2011). Mapping the moral domain. Journal of Personality and Social Psychology, 101(2), 366-385. https://doi.org/10.1037/a0021847

Graham, M., Hale, S. A., \& Gaffney, D. (2014). Where in the world are you? Geolocation and language identification in twitter. The Professional Geographer, 66(4), 568-578. https://doi.org/10.1080/00330124.2014.907699

Greaves, L. M., Osborne, D., \& Sibley, C. G. (2015). Profiling the fence-sitters in New Zealand elections: A latent profile model of political voting blocs. New Zealand Journal of Psychology, 44(2), 43-56.

Green Party of Aotearoa New Zealand. (n.d.). Agriculture and Rural Affairs Policy. Retrieved August 1, 2019, from https://www.greens.org.nz/page/agricultureand-rural-affairs-policy

Gregory, T. R. (2009). Understanding natural selection: Essential concepts and common misconceptions. Evolution: Education and Outreach, 2(2), 156-175. https://doi.org/10.1007/s12052-009-0128-1

Griffin, M., Shickle, D., \& Moran, N. (2008). European citizens' opinions on water fluoridation. Community Dentistry and Oral Epidemiology, 36(2), 95-102. https://doi.org/10.1111/j.1600-0528.2007.00373.x

Grzesiak-Feldman, M. (2015). Are the high authoritarians more prone to adopt conspiracy theories? The role of right-wing authoritarianism in conspiratorial thinking. In M. Bilewicz, A. Cichocka, \& W. Soral (Eds.), The Psychology of Conspiracy (pp. 117-139). https://doi.org/10.4324/9781315746838-13

Gummer, T., Roßmann, J., \& Silber, H. (2018). Using instructed response items as attention checks in web surveys. Sociological Methods \& Research, 004912411876908. https://doi.org/10.1177/0049124118769083

Hale, J. (2018, October 8). The Preference for "Natural." PsychCentral. Retrieved from https://psychcentral.com/lib/the-preference-for-natural/

Hall, C., \& Moran, D. (2006). Investigating GM risk perceptions: A survey of antiGM and environmental campaign group members. Journal of Rural Studies, 22(1), 29-37. https://doi.org/10.1016/J.JRURSTUD.2005.05.010

Hamburg, M. (2019). Transcending boundaries. Science, 363(6427), 563. https://doi.org/10.1126/science.aaw8238

Hamilton, L. C. (2016). Public awareness of the scientific consensus on climate. SAGE Open, 6(4), 1-11. https://doi.org/10.1177/2158244016676296

Hanna, M. (2016, December). Can the Greens ditch their anti-science baggage? 
Sciblogs. Retrieved from https://sciblogs.co.nz/honestuniverse/2016/12/15/cangreens-ditch-anti-science-baggage/

Hansson, S. O. (2009). Cutting the gordian knot of demarcation. International Studies in the Philosophy of Science, 23(3), 237-243. https://doi.org/10.1080/02698590903196007

Hansson, S. O. (2017). Science denial as a form of pseudoscience. Studies in History and Philosophy of Science Part A, 63, 39-47. https://doi.org/10.1016/J.SHPSA.2017.05.002

Harker, D. (2015). Creating Scientific Controversies. https://doi.org/10.1017/CBO9781107706903

Harnish, R. J., Bridges, K. R., \& Gump, J. T. (2018). Predicting economic, social, and foreign policy conservatism: The role of right-wing authoritarianism, social dominance orientation, moral foundations orientation, and religious fundamentalism. Current Psychology, 37(3), 668-679. https://doi.org/10.1007/s12144-016-9552-x

Hart, W., Albarracín, D., Eagly, A. H., Brechan, I., Lindberg, M. J., \& Merrill, L. (2009). Feeling validated versus being correct: A meta-analysis of selective exposure to information. Psychological Bulletin, 135(4), 555-588. https://doi.org/10.1037/a0015701

Hartman, R. O., Dieckmann, N. F., Sprenger, A. M., Stastny, B. J., \& DeMarree, K. G. (2017). Modeling attitudes toward science: Development and validation of the credibility of science scale. Basic and Applied Social Psychology, 39(6), 358-371. https://doi.org/10.1080/01973533.2017.1372284

Harvey, D. (2019). Helping you find reliable public health information on Twitter. Retrieved August 1, 2019, from Twitter website: https://blog.twitter.com/en_us/topics/company/2019/helping-you-find-reliablepublic-health-information-on-twitter.html

Hauser, D. J., \& Schwarz, N. (2016). Attentive Turkers: MTurk participants perform better on online attention checks than do subject pool participants. Behavior Research Methods, 48(1), 400-407. https://doi.org/10.3758/s13428-015-0578-z

Hayes, A. F. (2009). Beyond Baron and Kenny: Statistical mediation analysis in the new millennium. Communication Monographs, 76(4), 408-420. https://doi.org/10.1080/03637750903310360

Heath, Y., \& Gifford, R. (2006). Free-market ideology and environmental degradation. Environment and Behavior, 38(1), 48-71. https://doi.org/10.1177/0013916505277998

Heaven, P. C., \& Connors, J. R. (2001). A note on the value correlates of social dominance orientation and right-wing authoritarianism. Personality and Individual Differences, 31(6), 925-930. https://doi.org/10.1016/S01918869(00)00194-X

Helmi, M., Kate Spinella, M., \& Seymour, B. (2018). Community water fluoridation online: an analysis of the digital media ecosystem. Journal of Public Health 
Dentistry, 78(4), 296-305. https://doi.org/10.1111/jphd.12268

Hennes, E. P., Ruisch, B. C., Feygina, I., Monteiro, C. A., \& Jost, J. T. (2016).

Motivated recall in the service of the economic system: The case of anthropogenic climate change. Journal of Experimental Psychology: General, 145(6), 755-771. https://doi.org/10.1037/xge0000148

Ho, A. K., Sidanius, J., Kteily, N., Sheehy-Skeffington, J., Pratto, F., Henkel, K. E., ... Stewart, A. L. (2015). The nature of social dominance orientation: Theorizing and measuring preferences for intergroup inequality using the new $\mathrm{SDO}_{7}$ scale. Journal of Personality and Social Psychology, 109(6), 1003-1028. https://doi.org/10.1037/pspi0000033

Hoban, T. J. (2004). Global social acceptance of plant biotechnology. In P. Christou \& H. Klee (Eds.), Handbook of Plant Biotechnology. https://doi.org/10.1002/0470869143.kc059

Hong, S.-M., \& Page, S. (1989). A psychological reactance scale: development, factor structure and reliability. Psychological Reports, 64(3_suppl), 1323-1326. https://doi.org/10.2466/pr0.1989.64.3c.1323

Hornsey, M. J., \& Fielding, K. S. (2017). Attitude roots and Jiu Jitsu persuasion: Understanding and overcoming the motivated rejection of science. American Psychologist, 72(5), 459-473. https://doi.org/10.1037/a0040437

Hornsey, M. J., Harris, E. A., Bain, P. G., \& Fielding, K. S. (2016). Meta-analyses of the determinants and outcomes of belief in climate change. Nature Climate Change, 6(6), 622-626. https://doi.org/10.1038/nclimate2943

Hornsey, M. J., Harris, E. A., \& Fielding, K. S. (2018a). Relationships among conspiratorial beliefs, conservatism and climate scepticism across nations. Nature Climate Change, 8(7), 614-620. https://doi.org/10.1038/s41558-018$0157-2$

Hornsey, M. J., Harris, E. A., \& Fielding, K. S. (2018b). The Psychological Roots of Anti-Vaccination Attitudes: A 24-Nation Investigation. Health Psychology, 37(4), 307-315. https://doi.org/10.1037/hea0000586.supp

Hu, L., \& Bentler, P. M. (1999). Cutoff criteria for fit indexes in covariance structure analysis: Conventional criteria versus new alternatives. Structural Equation Modeling: A Multidisciplinary Journal, 6(1), 1-55. https://doi.org/10.1080/10705519909540118

Huang, B., \& Carley, K. M. (2017). On predicting geolocation of tweets using convolutional neural networks. Social, Cultural, and Behavioral Modeling, 10th International Conference, 281-291. https://doi.org/10.1007/978-3-319-602400_34

Huesing, J. E., Andres, D., Braverman, M. P., Burns, A., Felsot, A. S., Harrigan, G. G., ... Eloff, J. N. (2016). Global adoption of genetically modified (GM) crops: Challenges for the public sector. Journal of Agricultural and Food Chemistry, 64(2), 394-402. https://doi.org/10.1021/acs.jafc.5b05116

Hunsberger, B. (1996). Religious fundamentalism, right-wing authoritarianism, and 
hostility toward homosexuals in non-christian religious groups. International Journal for the Psychology of Religion, 6(1), 39-49.

https://doi.org/10.1207/s15327582ijpr0601_5

Institute of Medicine. (2012). Adverse Effects of Vaccines: Evidence and causality. https://doi.org/10.17226/13164

IPCC. (2014). IPCC Fifth Assessment Synthesis Report-Climate Change 2014 Synthesis Report. Retrieved from http://www.ipcc.ch/report/ar5/syr/

Irzik, G., \& Kurtulmus, F. (2018). What is epistemic public trust in science? The British Journal for the Philosophy of Science. https://doi.org/10.1093/bjps/axy007

Jacquet, J., Dietrich, M., \& Jost, J. T. (2014). The ideological divide and climate change opinion: "top-down" and "bottom-up" approaches. Frontiers in Psychology, 5, 1458. https://doi.org/10.3389/fpsyg.2014.01458

Jahn, D., Düpont, N., \& Rachuj, M. (2018). Left-Right Party Ideology in 36 Countries (No. 11). Retrieved from http://comparativepolitics.unigreifswald.de/gcp/GCP-11-2018.pdf

Jamieson, K. H., \& Hardy, B. W. (2014). Leveraging scientific credibility about Arctic sea ice trends in a polarized political environment. Proceedings of the National Academy of Sciences of the United States of America, 111 Suppl 4(Supplement 4), 13598-13605. https://doi.org/10.1073/pnas.1320868111

John, S. (2018). Epistemic trust and the ethics of science communication: against transparency, openness, sincerity and honesty. Social Epistemology, 32(2), 7587. https://doi.org/10.1080/02691728.2017.1410864

Johnson, B. B. (2017). "Counting votes" in public responses to scientific disputes. Public Understanding of Science, 1-17. https://doi.org/10.1177/0963662517706451

Jones, N. M., Wojcik, S. P., Sweeting, J., \& Silver, R. C. (2016). Tweeting negative emotion: An investigation of Twitter data in the aftermath of violence on college campuses. Psychological Methods, 21(4), 526-541. https://doi.org/10.1037/met0000099

Jose, P. E. (2013). Doing Statistical Mediation and Moderation. New York, NY: Guilford Press.

Jost, J. T. (2017). Ideological asymmetries and the essence of political psychology. Political Psychology, 38(2), 167-208. https://doi.org/10.1111/pops.12407

Jost, J. T., Blount, S., Pfeffer, J., \& Hunyady, G. (2003). Fair market ideology: Its cognitive-motivational underpinnings. Research in Organizational Behavior, 25, 53-91. https://doi.org/10.1016/S0191-3085(03)25002-4

Jost, J. T., Kruglanski, A. W., Glaser, J., Sulloway, F. J., Greenberg, J., Higgins, E. T., ... Sidanjus, J. (2003). Political conservatism as motivated social cognition. Psychological Bulletin, 129(3), 339-375. https://doi.org/http://dx.doi.org/10.1037/0033-2909.129.3.339 
Jost, J. T., \& Thompson, E. P. (2000). Group-based dominance and opposition to equality as independent predictors of self-esteem, ethnocentrism, and social policy attitudes among African Americans and European Americans. Journal of Experimental Social Psychology, 36(3), 209-232. https://doi.org/10.1006/JESP.1999.1403

Judge, M., \& Wilson, M. S. (2019). A dual-process motivational model of attitudes towards vegetarians and vegans. European Journal of Social Psychology, 49(1), 169-178. https://doi.org/10.1002/ejsp.2386

Jylhä, K. M., \& Akrami, N. (2015). Social dominance orientation and climate change denial: The role of dominance and system justification. Personality and Individual Differences, 86, 108-111. https://doi.org/10.1016/J.PAID.2015.05.041

Kahan, D. M. (2010). Fixing the communications failure. Nature, 463(7279), 296297. https://doi.org/doi: 10.1038/463296a

Kahan, D. M. (2012a). Cultural cognition as a conception of the cultural theory of risk. In S. Roeser, R. Hillerbrand, P. Sandin, \& M. Peterson (Eds.), Handbook of Risk Theory: Epistemology, Decision Theory, Ethics, and Social Implications of Risk (pp. 725-759). https://doi.org/10.1007/978-94-007-1433-5_28

Kahan, D. M. (2012b, September). Culturally polarized Australia: Cross-cultural cultural cognition, Part 3 (and a short diatribe about ugly regression outputs). Retrieved July 30, 2019, from Cultural Cognition Project website: http://www.culturalcognition.net/blog/2012/9/10/culturally-polarized-australiacross-cultural-cultural-cogni.html

Kahan, D. M. (2015). Climate-science communication and the measurement problem. Political Psychology, 36(S1), 1-43. https://doi.org/10.1111/pops.12244

Kahan, D. M. (2017). The "Gateway Belief" illusion: reanalyzing the results of a scientific-consensus messaging study. Journal of Science Communication, 15(05), A03.

Kahan, D. M., \& Carpenter, K. (2017). Out of the lab and into the field. Nature Climate Change, 7(5), 309-311. https://doi.org/10.1038/nclimate3283

Kahan, D. M., Jenkins-Smith, H., \& Braman, D. (2011). Cultural cognition of scientific consensus. Journal of Risk Research, 14(2), 147-174. https://doi.org/10.1080/13669877.2010.511246

Kahan, D. M., Peters, E., Wittlin, M., Slovic, P., Ouellette, L. L., Braman, D., \& Mandel, G. (2012). The polarizing impact of science literacy and numeracy on perceived climate change risks. Nature Climate Change, 2(10), 732-735. https://doi.org/10.1038/nclimate1547

Kassarjian, H. H., \& Cohen, J. B. (1965). Cognitive dissonance and consumer behavior. California Management Review, 8(1), 55-64. https://doi.org/10.2307/41165660

Kata, A. (2010). A postmodern Pandora's box: Anti-vaccination misinformation on 
the Internet. Vaccine, 28(7), 1709-1716.

https://doi.org/10.1016/J.VACCINE.2009.12.022

Kata, A. (2012). Anti-vaccine activists, Web 2.0, and the postmodern paradigm - An overview of tactics and tropes used online by the anti-vaccination movement. Vaccine, 30(25), 3778-3789. https://doi.org/10.1016/J.VACCINE.2011.11.112

Katz, D. (1960). The Functional Approach to the Study of Attitudes. Public Opinion Quarterly, 24(2, Special Issue: Attitude Change), 163. https://doi.org/10.1086/266945

Kearney, M. W. (2019). rtweet: Collecting Twitter Data (R package). Retrieved July 4, 2019, from https://cran.r-project.org/package=rtweet

Kern, M. L., Park, G., Eichstaedt, J. C., Schwartz, H. A., Sap, M., Smith, L. K., \& Ungar, L. H. (2016). Gaining insights from social media language:

Methodologies and challenges. Psychological Methods, 21(4), 507-525. https://doi.org/10.1037/met0000091

Kerr, J. R., \& Wilson, M. S. (2018a). Changes in perceived scientific consensus shift beliefs about climate change and GM food safety. PLOS ONE, 13(7), e0200295. https://doi.org/10.1371/journal.pone.0200295

Kerr, J. R., \& Wilson, M. S. (2018b). Perceptions of scientific consensus do not predict later beliefs about the reality of climate change: A test of the gateway belief model using cross-lagged panel analysis. Journal of Environmental Psychology, 59, 107-110. https://doi.org/10.1016/J.JENVP.2018.08.012

Kim, K. S. (2011). Public understanding of the politics of global warming in the news media: the hostile media approach. Public Understanding of Science, 20(5), 690-705. https://doi.org/10.1177/0963662510372313

Kirk, R. E. (1996). Practical significance: A concept whose time has come.

Educational and Psychological Measurement, 56(5), 746-759.

https://doi.org/10.1177/0013164496056005002

Knight, K. (2006). Transformations of the concept of ideology in the twentieth century. American Political Science Review, 100(4). https://doi.org/10.1017/S0003055406062502

Knox, M. C., Garner, A., Dyason, A., Pearson, T., \& Pit, S. W. (2017). Qualitative investigation of the reasons behind opposition to water fluoridation in regional NSW, Australia. Public Health Research \& Practice, 27(1). https://doi.org/10.17061/phrp2711705

Kobayashi, K. (2018). The impact of perceived scientific and social consensus on scientific beliefs. Science Communication, 40(1), 63-88. https://doi.org/10.1177/1075547017748948

Koehler, D. J. (2016). Can journalistic "false balance" distort public perception of consensus in expert opinion? Journal of Experimental Psychology: Applied, 22(1), 24-38. https://doi.org/10.1037/xap0000073

Kohl, P. A., Kim, S. Y., Peng, Y., Akin, H., Koh, E. J., Howell, A., \& Dunwoody, S. (2016). The influence of weight-of-evidence strategies on audience perceptions 
of (un)certainty when media cover contested science. Public Understanding of Science, 25(8), 976-991. https://doi.org/10.1177/0963662515615087

Krosnick, J. A., \& Alwin, D. F. (1989). Aging and susceptibility to attitude change. Journal of Personality and Social Psychology, 57(3), 416-425.

Kruglanski, A. W., \& Stroebe, W. (2005). The influence of beliefs and goals on attitudes: Issues of structure, function, and dynamics. In D. Albarracin, B. T. Johnson, \& M. P. Zanna (Eds.), The Handbook of Attitudes (pp. 323-268). Lawrence Erlbaum, Mahwah, NJ.

Kunda, Z. (1987). Motivated inference: Self-serving generation and evaluation of causal theories. Journal of Personality and Social Psychology, 53(4), 636-647. https://doi.org/10.1037/0022-3514.53.4.636

Kunda, Z. (1990). The case for motivated reasoning. Psychological Bulletin, 108(3), 480-498. https://doi.org/10.1037/0033-2909.108.3.480

Lang, J. T. (2016). What's so controversial about genetically modified food? London: Reaktion Books.

Langman, L. (2017). The social psychology of critical theory. In M. J. Thompson (Ed.), The Palgrave Handbook of Critical Theory (pp. 443-462). https://doi.org/10.1057/978-1-137-55801-5_20

Larson, H. J., de Figueiredo, A., Xiahong, Z., Schulz, W. S., Verger, P., Johnston, I. G., ... Jones, N. S. (2016). The state of vaccine confidence 2016: global insights through a 67-country survey. EBioMedicine, 12, 295-301. https://doi.org/10.1016/j.ebiom.2016.08.042

Larson, R. B. (2018). Examining consumer attitudes toward genetically modified and organic foods. British Food Journal, 120(5), 999-1014. https://doi.org/10.1108/BFJ-09-2017-0502

Lazer, D. M. J., Baum, M. A., Benkler, Y., Berinsky, A. J., Greenhill, K. M., Menczer, F., ... Zittrain, J. L. (2018). The science of fake news. Science, 359(6380), 1094-1096. https://doi.org/10.1126/science.aao2998

Lee, C. H. J., Duck, I. M., \& Sibley, C. G. (2017). Personality and demographic correlates of New Zealanders' confidence in the safety of childhood vaccinations. Vaccine, 35(45), 6089-6095. https://doi.org/10.1016/J.VACCINE.2017.09.061

Leining, C., \& White, S. (2015). From Fact to Act: New Zealanders' Beliefs and Actions on Climate Change. Retrieved from https://motu.nz/ourwork/environment-and-resources/emission-mitigation/shaping-new-zealandslow-emissions-future/from-fact-to-act-new-zealanders-beliefs-and-actions-onclimate-change/

Leiserowitz, A. (2006). Climate change risk perception and policy preferences: The role of affect, imagery, and values. Climatic Change, 77(1-2), 45-72. https://doi.org/10.1007/s10584-006-9059-9

Lewandowsky, S., Cook, J., \& Ecker, U. K. H. (2017). Letting the gorilla emerge from the mist: Getting past post-truth. Journal of Applied Research in Memory 
and Cognition, 6(4), 418-424. https://doi.org/10.1016/J.JARMAC.2017.11.002

Lewandowsky, S., Cook, J., \& Lloyd, E. (2018). The 'Alice in Wonderland' mechanics of the rejection of (climate) science: simulating coherence by conspiracism. Synthese, 195(1), 175-196. https://doi.org/10.1007/s11229-016$1198-6$

Lewandowsky, S., Ecker, U. K. H., \& Cook, J. (2017). Beyond misinformation: Understanding and coping with the "post-truth" era. Journal of Applied Research in Memory and Cognition, 6(4), 353-369. https://doi.org/10.1016/J.JARMAC.2017.07.008

Lewandowsky, S., Gignac, G. E., \& Oberauer, K. (2013). The role of conspiracist ideation and worldviews in predicting rejection of science. PLOS ONE, 8(10), e75637. https://doi.org/10.1371/journal.pone.0075637

Lewandowsky, S., Gignac, G. E., \& Vaughan, S. (2012). The pivotal role of perceived scientific consensus in acceptance of science. Nature Climate Change, 3(4), 399-404. https://doi.org/10.1038/nclimate1720

Lewandowsky, S., Mann, M. E., Brown, N. J. L., Friedman, H., \& Friedman, H. (2016). Science and the public: Debate, denial, and skepticism. Journal of Social and Political Psychology, 4(2), 537-553. https://doi.org/10.5964/jspp.v4i2.604

Lewandowsky, S., \& Oberauer, K. (2016). Motivated rejection of science. Current Directions in Psychological Science, 25(4), 217-222. https://doi.org/10.1177/0963721416654436

Lewandowsky, S., Oberauer, K., \& Gignac, G. E. (2013). NASA faked the moon landing - therefore, (climate) science is a hoax. Psychological Science, 24(5), 622-633. https://doi.org/10.1177/0956797612457686

Li, Y., Johnson, E. J., \& Zaval, L. (2011). Local warming: daily temperature change influences belief in global warming. Psychological Science, 22(4), 454-459. https://doi.org/10.1177/0956797611400913

Little, T. D., Cunningham, W. A., Shahar, G., \& Widaman, K. F. (2009). To parcel or not to parcel: Exploring the question, weighing the merits. Structural Equation Modeling, 9(2), 151-173. https://doi.org/10.1207/S15328007SEM0902_1

Lobato, E. J. C., \& Zimmerman, C. (2019). Examining how people reason about controversial scientific topics. Thinking \& Reasoning, 25(2), 231-255. https://doi.org/10.1080/13546783.2018.1521870

Lobato, E., Mendoza, J., Sims, V., \& Chin, M. (2014). Examining the relationship between conspiracy theories, paranormal beliefs, and pseudoscience acceptance among a university population. Applied Cognitive Psychology, 28(5), 617-625. https://doi.org/10.1002/acp.3042

Louisson, S. (2016, May). Silencing scientists integral to National's anti-science DNA. The Standard. Retrieved from https://thestandard.org.nz/silencingscientists-integral-to-nationals-anti-science-dna/ 
Lucht, J. M. (2015). Public acceptance of plant biotechnology and GM crops. Viruses, 7(8), 4254-4281. https://doi.org/10.3390/v7082819

Lull, R. B., \& Scheufele, D. A. (2017). Understanding and overcoming fear of the unnatural in discussion of GMOs. In K. H. Jamieson, D. M. Kahan, \& D. A. Scheufele (Eds.), The Oxford Handbook of the Science of Science Communication. https://doi.org/10.1093/oxfordhb/9780190497620.013.44

Ma, Y., Dixon, G., \& Hmielowski, J. D. (2019). Psychological reactance from reading basic facts on climate change: The role of prior views and political identification. Environmental Communication, 13(1), 71-86. https://doi.org/10.1080/17524032.2018.1548369

Maibach, E., Leiserowitz, A., Roser-Renouf, C., Myers, T., Rosenthal, S., \& Feinberg, G. (2015). The Francis Effect: How Pope Francis Changed the Conversation about Global Warming. Retrieved from https://www.climatechangecommunication.org/all/thefranciseffect/

Maibach, E., Myers, T., \& Leiserowitz, A. (2014). Climate scientists need to set the record straight: There is a scientific consensus that human-caused climate change is happening. Earth's Future, 2(5), 295-298. https://doi.org/10.1002/2013EF000226

Maibach, E., \& van der Linden, S. L. (2016). The importance of assessing and communicating scientific consensus. Environmental Research Letters, 11(9), 091003. https://doi.org/10.1088/1748-9326/11/9/091003

Marques, M. D., Critchley, C. R., \& Walshe, J. (2015). Attitudes to genetically modified food over time: How trust in organizations and the media cycle predict support. Public Understanding of Science, 24(5), 601-618. https://doi.org/10.1177/0963662514542372

Martin, B. (2015). Censorship and free speech in scientific controversies. Science and Public Policy, 42(3), 377-386. https://doi.org/10.1093/scipol/scu061

Martin, J. L. (2001). The Authoritarian Personality 50 years later: What lessons are there for political psychology? Political Psychology, 22(1), 1-26. https://doi.org/http://dx.doi.org/10.1111/0162-895X.00223

Matthews, M., Levin, S., \& Sidanius, J. (2009). A longitudinal test of the model of political conservatism as motivated social cognition. Political Psychology, 30(6), 921-936. https://doi.org/10.1111/j.1467-9221.2009.00733.x

Mavor, K. I., Louis, W. R., \& Laythe, B. (2011). Religion, prejudice, and authoritarianism: Is RWA a boon or bane to the psychology of religion? Journal for the Scientific Study of Religion, 50(1), 22-43. https://doi.org/https://doi.org/10.1111/j.1468-5906.2010.01550.x

McCoy, C. A. (2018). The social characteristics of Americans opposed to vaccination: beliefs about vaccine safety versus views of U.S. vaccination policy. Critical Public Health, 1-12. https://doi.org/10.1080/09581596.2018.1501467

McCright, A. M., Dentzman, K., Charters, M., \& Dietz, T. (2013). The influence of 
political ideology on trust in science. Environmental Research Letters, 8(4), 044029. https://doi.org/10.1088/1748-9326/8/4/044029

McCright, A. M., \& Dunlap, R. E. (2011). Cool dudes: The denial of climate change among conservative white males in the United States. Global Environmental Change, 21(4), 1163-1172. https://doi.org/10.1016/J.GLOENVCHA.2011.06.003

McCright, A. M., Dunlap, R. E., \& Xiao, C. (2013). Perceived scientific agreement and support for government action on climate change in the USA. Climatic Change, 119(2), 511-518. https://doi.org/https://doi.org/10.1007/s10584-0130704-9

McFadden, B. R. (2016). Examining the gap between science and public opinion about genetically modified food and global warming. PLOS ONE, 11(11), e0166140. https://doi.org/10.1371/journal.pone.0166140

Mcfarland, S. G. (2005). On the eve of war: Authoritarianism, social dominance, and American students' attitudes toward attacking Iraq. Personality and Social Psychology Bulletin, 31(3), 360-367. https://doi.org/10.1177/0146167204271596

McPhetres, J., Rutjens, B. T., Weinstein, N., \& Brisson, J. A. (2019). Modifying attitudes about modified foods: Increased knowledge leads to more positive attitudes. Journal of Environmental Psychology, 64, 21-29. https://doi.org/10.1016/J.JENVP.2019.04.012

McPhetres, J., \& Zuckerman, M. (2018). Religiosity predicts negative attitudes towards science and lower levels of science literacy. PLOS ONE, 13(11), e0207125. https://doi.org/10.1371/journal.pone.0207125

Medvecky, F., \& Leach, J. (2017). The ethics of science communication. Journal of Science Communication, 16(4). https://doi.org/10.22323/2.16040501

Menczer, F., \& Hui, P.-M. (2019, April 2). Anti-vaxxers appear to be losing ground in the online vaccine debate. The Conversation. Retrieved from https://theconversation.com/anti-vaxxers-appear-to-be-losing-ground-in-theonline-vaccine-debate-114406

Meningitis Foundation Aotearoa New Zealand. (2013). Immunisation Health Report. Retrieved from http://www.pfizer.co.nz/sites/nzbrandsite.pfizer.edrupalgardens.com/files/20130 7/Immunisation Health Report web.pdf

Milfont, T. L. (2009). The effects of social desirability on self-reported environmental attitudes and ecological behaviour. The Environmentalist, 29(3), 263-269. https://doi.org/10.1007/s10669-008-9192-2

Milfont, T. L., Milojev, P., Greaves, L. M., \& Sibley, C. G. (2015). Socio-structural and psychological foundations of climate change beliefs. New Zealand Journal of Psychology, 44(1), 17-30.

Milfont, T. L., Richter, I., Sibley, C. G., Wilson, M. S., \& Fischer, R. (2013). Environmental consequences of the desire to dominate and be superior. 
Personality and Social Psychology Bulletin, 39(9), 1127-1138.

https://doi.org/10.1177/0146167213490805

Milfont, T. L., Wilson, M. S., \& Sibley, C. G. (2017). The public's belief in climate change and its human cause are increasing over time. PLOS ONE, 12(3), e0174246. https://doi.org/10.1371/journal.pone.0174246

Miller, J. D. (2004). Public understanding of, and attitudes toward, scientific research: What we know and what we need to know. Public Understanding of Science, 13(3), 273-294. https://doi.org/10.1177/0963662504044908

Miller, J. D., Scott, E. C., \& Okamoto, S. (2006). Public acceptance of evolution. Science, 313(5788), 765-766. https://doi.org/10.1126/science.1126746

Ministry for the Environment. (n.d.). Genetic modification in New Zealand. Retrieved March 21, 2019, from http://www.mfe.govt.nz/publications/hazards/gm-nz-approach-jun04/geneticmodification-new-zealand

Ministry of Health. (2018). Immunisation Handbook 2017 (2nd ed.). Retrieved from www.health.govt.nz

Mishra, V. (2017). GMOs Insights Analysis: May-June 2017. Retrieved June 25, 2019, from http://info.rightrelevance.com/gmos-insights-analysis-may-june$2017 /$

Mitra, T., Counts, S., \& Pennebaker, J. W. (2016). Understanding anti-vaccination attitudes in social media. Tenth International AAAI Conference on Web and Social Media, 269-278. Retrieved from https://www.aaai.org/ocs/index.php/ICWSM/ICWSM16/paper/viewPaper/1307 3

Mooney, C. (2012). The Republican brain: the science of why they deny science and reality. Hoboken, NJ: John Wiley \& Sons, Inc.

Morgan, M., Collins, W., Sparks, G., \& Welch, J. (2018). Identifying relevant antiscience perceptions to improve science-based communication: The Negative Perceptions of Science Scale. Social Sciences, 7(4), 64. https://doi.org/10.3390/socsci7040064

Mortimer, K. (2017). Understanding conspiracy online: Social media and the spread of suspicious thinking. Dalhousie Journal of Interdisciplinary Management, 13(1). https://doi.org/10.5931/djim.v13i1.6928

Morton, J. (2017, December 3). Does NZ really have a science denial problem? - NZ Herald. New Zealand Herald. Retrieved from https://www.nzherald.co.nz/nz/news/article.cfm?c_id=1\&objectid=11952249

Moscovici, S., \& Mugny, G. (1983). Minority influence. In P. B. Paulus (Ed.), Basic Group Proceses (pp. 41-64). Retrieved from https://link.springer.com/content/pdf/10.1007\%2F978-1-4612-5578-9_3.pdf

Myers, T. A., Maibach, E., Peters, E., \& Leiserowitz, A. (2015). Simple messages help set the record straight about scientific agreement on human-caused climate change: The results of two experiments. PLOS ONE, 10(3). 
https://doi.org/10.1371/journal.pone.0120985

Myers, T. A., Maibach, E. W., Roser-Renouf, C., Akerlof, K., \& Leiserowitz, A. A. (2013). The relationship between personal experience and belief in the reality of global warming. Nature Climate Change, 3(4), 343-347.

https://doi.org/10.1038/nclimate1754

Nadelson, L. S., \& Hardy, K. K. (2015). Trust in science and scientists and the acceptance of evolution. Evolution: Education and Outreach, 8(1), 9. https://doi.org/10.1186/s12052-015-0037-4

Nadelson, L. S., Jorcyk, C., Yang, D., Jarratt Smith, M., Matson, S., Cornell, K., \& Husting, V. (2014). I just don't trust them: The development and validation of an assessment instrument to measure trust in science and scientists. School Science and Mathematics, 114(2), 76-86. https://doi.org/10.1111/ssm.12051

National Academies of Sciences, Engineering, and Medicine. (2016a). Communicating Science Effectively: A Research Agenda Science. https://doi.org/10.17226/23674

National Academies of Sciences, Engineering, and Medicine. (2016b). Genetically Engineered Crops: Experiences and Prospects. https://doi.org/10.17226/23395.

National Academies of Sciences, Medicine, and Engineering. (2015). Trust and Confidence at the Interfaces of the Life Sciences and Society. A Workshop Summary. Retrieved from https://www.nap.edu/read/21798

National Fluoridation Information Service. (2014). Environmental Scan: The status of community water fluoridation in New Zealand March 2013 - July 2014. Retrieved from http://www.rph.org.nz/content/d8625992-82a9-4136-88a36b24da820916.cmr

Nauroth, P., Gollwitzer, M., Bender, J., \& Rothmund, T. (2015). Social identity threat motivates science-discrediting online comments. PLOS ONE, 10(2), e0117476. https://doi.org/10.1371/journal.pone.0117476

New Zealand First. (n.d.). About New Zealand First. Retrieved August 1, 2019, from https://www.nzfirst.org.nz/about

Newman, T. P. (2017). Tracking the release of IPCC AR5 on Twitter: Users, comments, and sources following the release of the Working Group I Summary for Policymakers. Public Understanding of Science, 26(7), 815-825. https://doi.org/10.1177/0963662516628477

Nicolia, A., Manzo, A., Veronesi, F., \& Rosellini, D. (2014). An overview of the last 10 years of genetically engineered crop safety research. Critical Reviews in Biotechnology, 34(1), 77-88. https://doi.org/10.3109/07388551.2013.823595

Nisbet, E. C., Cooper, K. E., \& Ellithorpe, M. (2015). Ignorance or bias? Evaluating the ideological and informational drivers of communication gaps about climate change. Public Understanding of Science, 24(3), 285-301. https://doi.org/10.1177/0963662514545909

Nisbet, M. C., \& Mooney, C. (2007). Framing science. Science, 316(5821), 56. https://doi.org/10.1126/science. 1142030 
NZ Parliament. (2015). Primary Production Committee report on Petition 2011/68 of Jon Muller. Retrieved from https://www.parliament.nz/resource/ennz/51DBSCH_SCR62256_1/081c26f143ac1696ec551b82bad4e1f004985779

O’Neill, S. J., \& Boykoff, M. (2010). Climate denier, skeptic, or contrarian? Proceedings of the National Academy of Sciences of the United States of America, 107(39), E151; author reply E152. https://doi.org/10.1073/pnas.1010507107

O’Neill, S., Williams, H. T. P., Kurz, T., Wiersma, B., \& Boykoff, M. (2015). Dominant frames in legacy and social media coverage of the IPCC Fifth Assessment Report. Nature Climate Change, 5(4), 380-385. https://doi.org/10.1038/nclimate2535

O'Rourke, H. P., \& MacKinnon, D. P. (2015). When the test of mediation is more powerful than the test of the total effect. Behavior Research Methods, 47(2), 424. https://doi.org/10.3758/s13428-014-0481-z

Omer, S. B., \& Yildirim, I. (2019). Further evidence of MMR vaccine safety: Scientific and communications considerations. Annals of Internal Medicine. https://doi.org/10.7326/M19-0596

Omobowale, E. B., Singer, P. A., \& Daar, A. S. (2009). The three main monotheistic religions and GM food technology: An overview of perspectives. BMC International Health and Human Rights, 9(1), 18. https://doi.org/10.1186/1472698X-9-18

Onraet, E., Van Hiel, A., Dhont, K., Hodson, G., Schittekatte, M., \& De Pauw, S. (2015). The association of cognitive ability with right-wing ideological attitudes and prejudice: A meta-analytic review. European Journal of Personality, 29(6), 599-621. https://doi.org/10.1002/per.2027

Oreskes, N. (2015). The fact of uncertainty, the uncertainty of facts and the cultural resonance of doubt. Philosophical Transactions of the Royal Society A: Mathematical, Physical and Engineering Sciences, 373(2055), 20140455. https://doi.org/10.1098/rsta.2014.0455

Oreskes, N. (2017). Response by Oreskes to "Beyond Counting Climate Consensus." Environmental Communication, 11(6), 731-732. https://doi.org/10.1080/17524032.2017.1377094

Oreskes, N., \& Conway, E. M. (2010). Merchants of doubt. London: Bloomsbury Press.

Oxford Dictionaries. (n.d.). Word of the Year: frequently asked questions. Retrieved September 2, 2019, from https://languages.oup.com/word-of-the-year/word-ofthe-year-faqs

Ozawa, S., Clark, S., Portnoy, A., Grewal, S., Stack, M. L., Sinha, A., ... Walker, D. (2017). Estimated economic impact of vaccinations in 73 low-and middleincome countries, 2001-2020. Bulletin of the World Health Organization, 95(9), 629-638. https://doi.org/10.2471/BLT.16.178475

Paarlberg, R. (2015). Consequences of the Anti-GMO Campaigns. In Antonio M. 
Battro, Ingo Potrykus, \& Marcelo Sánchez Sorondo (Eds.), Bread and Brain, Education and Poverty. Retrieved from http://www.pas.va/content/dam/accademia/pdf/sv125/sv125-paarlberg.pdf

Parliamentary Commissioner for the Environment. (2016). Climate change and agriculture: Understanding the biological greenhouse gases. Retrieved from http://www.pce.parliament.nz/media/1678/climate-change-and-agricultureweb.pdf

Pasek, J. (2017). It's not my consensus: Motivated reasoning and the sources of scientific illiteracy. Public Understanding of Science, 1-20. https://doi.org/10.1177/0963662517733681

Pasek, J. (2018). Don't trust the scientists! Rejecting the scientific consensus "conspiracy." In Conspiracy Theories and the People Who Believe Them (pp. 201-213). https://doi.org/10.1093/oso/9780190844073.003.0013

Paynter, J. (2018). National Immunisation Coverage for New Zealand. Retrieved from http://www.immune.org.nz/sites/default/files/publications/Immunisation coverage to June 2018.pdf

Pearce, W., Grundmann, R., Hulme, M., Raman, S., Hadley Kershaw, E., \& Tsouvalis, J. (2017a). A reply to Cook and Oreskes on climate science consensus messaging. Environmental Communication, 11(6), 736-739. https://doi.org/10.1080/17524032.2017.1392109

Pearce, W., Grundmann, R., Hulme, M., Raman, S., Hadley Kershaw, E., \& Tsouvalis, J. (2017b). Beyond counting climate consensus. Environmental Communication, 1-8. https://doi.org/10.1080/17524032.2017.1333965

Peters, G., \& Heinemann, J. (2012). Should NZ grow genetically modified crops? NZ Herald. Retrieved August 23, 2017, from New Zealand Herald website: http://www.nzherald.co.nz/business/news/article.cfm?c_id=3\&objectid=108333 17

Petousis-Harris, H. (2019, August 21). Why is there a rip-roaring measles outbreak in NZ? Sciblogs. Retrieved from https://sciblogs.co.nz/diplomaticimmunity/2019/08/21/why-is-there-a-riproaring-measles-outbreak-in-nz

Petty, R. E., \& Cacioppo, J. T. (1986). The Elaboration Likelihood Model of Persuasion. In L. Berkowitz (Ed.), Advances in Experimental Social Psychology (Vol 19, pp. 123-205). https://doi.org/10.1016/S0065-2601(08)60214-2

Phadke, V. K., Bednarczyk, R. A., Salmon, D. A., \& Omer, S. B. (2016). Association between vaccine refusal and vaccine-preventable diseases in the United States. JAMA, 315(11), 1149. https://doi.org/10.1001/jama.2016.1353

Pielke, R. A. (2007). The Honest Broker: Making Sense of Science in Policy and Politics. Cambridge: Cambridge University Press.

Pigliucci, M., Baum, D., \& Mcpeek, M. (2006). Countering the Wedge: A multipronged, multi-year strategy to oppose creationism and intelligent design in the science curriculum of public schools. Retrieved from 
http://www.oklascience.com/anti-wedge_document.pdf

Pizzo, G., Piscopo, M. R., Pizzo, I., \& Giuliana, G. (2007). Community water fluoridation and caries prevention: a critical review. Clinical Oral Investigations, 11(3), 189-193. https://doi.org/10.1007/s00784-007-0111-6

Plante, T. G., Vallaeys, C. L., Sherman, A. C., \& Wallston, K. A. (2002). The development of a brief version of the Santa Clara Strength of Religious Faith Questionnaire. Pastoral Psychology, 50(5), 359-368. https://doi.org/10.1023/A:1014413720710

Podsakoff, P. M., MacKenzie, S. B., Lee, J.-Y., \& Podsakoff, N. P. (2003). Common method biases in behavioral research: A critical review of the literature and recommended remedies. Journal of Applied Psychology, 88(5), 879-903. https://doi.org/10.1037/0021-9010.88.5.879

Poland, G. A., \& Jacobson, R. M. (2011). The age-old struggle against the antivaccinationists. New England Journal of Medicine, 364(2), 97-99. https://doi.org/10.1056/NEJMp1010594

Polkinghorne, J. (2016). Guest post: carbon taxes and the NZ economy - Greater Auckland. Retrieved August 23, 2017, from https://www.greaterauckland.org.nz/2016/11/17/guest-post-carbon-taxes-andthe-nz-economy/

Poole, K. T., \& Rosenthal, H. (2001). D-Nominate after 10 years: a comparative update to congress: A political-economic history of roll-call voting. Legislative Studies Quarterly, 26(1), 5-29. https://doi.org/10.2307/440401

Poortinga, W., Spence, A., Whitmarsh, L., Capstick, S., \& Pidgeon, N. F. (2011). Uncertain climate: An investigation into public scepticism about anthropogenic climate change. Global Environmental Change, 21(3), 1015-1024. https://doi.org/10.1016/J.GLOENVCHA.2011.03.001

Poulter, S. (2013, October 21). Scientists issue safety warning over GM food as Government pushes for public acceptance of controversial crop. Daily Mail. Retrieved from www.dailymail.co.uk/news/article-2469738/Scientists-issuesafety-warning-GM-food-Government-pushes-public-acceptance-controversialcrop.html

Pratto, F., Stallworth, L. M., Sidanius, J., \& Siers, B. (1997). The gender gap in occupational role attainment: A social dominance approach. Journal of Personality and Social Psychology, 72(1), 37-53. https://doi.org/10.1037/00223514.72.1.37

Preacher, K. J., \& Kelley, K. (2011). Effect size measures for mediation models: Quantitative strategies for communicating indirect effects. Psychological Methods, 16(2), 93-115. https://doi.org/10.1037/a0022658

Prot, S. (2015). Science denial as intergroup conflict: using social identity theory, intergroup emotions theory and intergroup threat theory to explain angry denial of science (Iowa State University). https://doi.org/10.31274/etd-180810-4531

Rabinowitz, M., Latella, L., Stern, C., \& Jost, J. T. (2016). Beliefs about childhood 
vaccination in the united states: Political ideology, false consensus, and the illusion of uniqueness. PLOS ONE, 11(7), e0158382.

https://doi.org/10.1371/journal.pone.0158382

Rad, M. S., Martingano, A. J., \& Ginges, J. (2018). Toward a psychology of Homo sapiens: Making psychological science more representative of the human population. Proceedings of the National Academy of Sciences of the United States of America, 115(45), 11401-11405. https://doi.org/10.1073/pnas.1721165115

Ranney, M. A., \& Clark, D. (2016). Climate change conceptual change: Scientific information can transform attitudes. Topics in Cognitive Science, 8(1), 49-75. https://doi.org/10.1111/tops.12187

Remy, E. (2019). How public and private Twitter users in the U.S. compare - and why it might matter for your research. Retrieved September 23, 2019, from Medium website: https://medium.com/pew-research-center-decoded/howpublic-and-private-twitter-users-in-the-u-s-d536ce2a41b3

Ritter, R. S., Preston, J. L., \& Hernandez, I. (2014). Happy tweets: Christians are happier, more socially connected, and less analytical than atheists on Twitter. Social Psychological and Personality Science, 5(2), 243-249. https://doi.org/10.1177/1948550613492345

Rivers, C. M., \& Lewis, B. L. (2014). Ethical research standards in a world of big data. F1000Research, 38. https://doi.org/10.12688/f1000research.3-38.v2

Roccato, M. (2008). Right-wing authoritarianism, social dominance orientation, and attachment: An Italian study. Swiss Journal of Psychology, 67(4), 219-229. https://doi.org/10.1024/1421-0185.67.4.219

Roney, C. (2009). The psychology of free-market ideology: Links between economic and social conservatism. Paper Presented at the Annual Meeting of the ISPP 32nd Annual Scientific Meeting. Retrieved from http://citation.allacademic.com/meta/p_mla_apa_research_citation/3/0/5/3/9/p3 05391_index.html

Roos, J. M. (2014). Measuring science or religion? A measurement analysis of the National Science Foundation sponsored science literacy scale 2006-2010. Public Understanding of Science, 23(7), 797-813. https://doi.org/10.1177/0963662512464318

Rosenau, J. (2012). Science denial: a guide for scientists. Trends in Microbiology, 20(12), 567-569. https://doi.org/10.1016/J.TIM.2012.10.002

Rosseel, Y. (2012). lavaan : An R package for structural equation modeling. Journal of Statistical Software, 48(2), 1-36. https://doi.org/10.18637/jss.v048.i02

Rossen, I., Hurlstone, M. J., Dunlop, P. D., \& Lawrence, C. (2019). Accepters, fence sitters, or rejecters: Moral profiles of vaccination attitudes. Social Science \& Medicine, 224, 23-27. https://doi.org/10.1016/J.SOCSCIMED.2019.01.038

Royal Society of New Zealand. (2014). Health effects of water fluoridation: A review of the scientific evidence. Retrieved from https://royalsociety.org.nz/what-we- 
do/our-expert-advice/all-expert-advice-papers/health-effects-of-waterfluoridation/

Royal Society of New Zealand. (2016). Transition to a low-carbon economy for New Zealand. Retrieved from https://royalsociety.org.nz/assets/documents/ReportTransition-to-Low-Carbon-Economy-for-NZ.pdf

Rozin, P., Spranca, M., Krieger, Z., Neuhaus, R., Surillo, D., Swerdlin, A., \& Wood, K. (2004). Preference for natural: instrumental and ideational/moral motivations, and the contrast between foods and medicines. Appetite, 43(2), 147-154. https://doi.org/10.1016/J.APPET.2004.03.005

Rucker, D. D., Preacher, K. J., Tormala, Z. L., \& Petty, R. E. (2011). Mediation analysis in social psychology: Current practices and new recommendations. Social and Personality Psychology Compass, 5(6), 359-371. https://doi.org/10.1111/j.1751-9004.2011.00355.x

Rudski, J. M., Osei, W., Jacobson, A. R., \& Lynch, C. R. (2011). Would you rather be injured by lightning or a downed power line? Preference for natural hazards. Judgment and Decision Making, 6(4), 314. Retrieved from http://journal.sjdm.org/11/10809/jdm10809.html

Rutjens, B. T., \& Heine, S. J. (2016). The immoral landscape? Scientists are associated with violations of morality. PLOS ONE, 11(4), e0152798. https://doi.org/10.1371/journal.pone.0152798

Rutjens, B. T., Heine, S. J., Sutton, R. M., \& van Harreveld, F. (2018). Attitudes towards science. Advances in Experimental Social Psychology, 57, 125-165. https://doi.org/10.1016/BS.AESP.2017.08.001

Rutjens, B. T., Sutton, R. M., \& van der Lee, R. (2017). Not all skepticism is equal: Exploring the ideological antecedents of science acceptance and rejection. Personality and Social Psychology Bulletin, 014616721774131. https://doi.org/10.1177/0146167217741314

Salmon, R., Priestley, R., Fontana, M., \& Milfont, T. L. (2017). Climate change communication in New Zealand. In Oxford Research Encyclopedia of Climate Science. https://doi.org/10.1093/acrefore/9780190228620.013.475

Sarathchandra, D., Navin, M. C., Largent, M. A., \& McCright, A. M. (2018). A survey instrument for measuring vaccine acceptance. Preventive Medicine, 109, 1-7. https://doi.org/10.1016/J.YPMED.2018.01.006

Scheitle, C. P., \& Ecklund, E. H. (2018). Perceptions of religious discrimination among U.S. scientists. Journal for the Scientific Study of Religion, 57(1), 139155. https://doi.org/10.1111/jssr.12503

Scheitle, C. P., Johnson, D. R., \& Ecklund, E. H. (2018). Scientists and religious leaders compete for cultural authority of science. Public Understanding of Science, 27(1), 59-75. https://doi.org/10.1177/0963662517718145

Schwartz, S. H. (1992). Universals in the content and structure of values: Theoretical advances and empirical tests in 20 countries. Advances in Experimental Social Psychology, 25, 1-65. https://doi.org/10.1016/S0065-2601(08)60281-6 
Schwarz, N., Newman, E., \& Leach, W. (2016). Making the truth stick \& the myths fade: Lessons from cognitive psychology. Behavioral Science \& Policy, 2(1), $85-95$.

Scott, E. C. (1997). Antievolution and creationism in the United States. Annual Review of Anthropology, 26(1), 263-289.

https://doi.org/10.1146/annurev.anthro.26.1.263

Sears, D. O. (1986). College sophomores in the laboratory: Influences of a narrow data base on social psychology's view of human nature. Journal of Personality and Social Psychology, 51(3), 515-530. https://doi.org/10.1037/00223514.51.3.515

Selig, J. P., \& Little, T. D. (2012). Autoregressive and cross-lagged panel analysis for longitudinal data. In B. Laursen, T. D. Little, \& N. A. Card (Eds.), Handbook of developmental research methods (pp. 265-278). New York: Guilford Press.

Shapiro, G. K., Holding, A., Perez, S., Amsel, R., \& Rosberger, Z. (2016). Validation of the vaccine conspiracy beliefs scale. Papillomavirus Research, 2, 167-172. https://doi.org/10.1016/J.PVR.2016.09.001

Shapiro, G. K., Tatar, O., Dube, E., Amsel, R., Knauper, B., Naz, A., ... Rosberger, Z. (2018). The vaccine hesitancy scale: Psychometric properties and validation. Vaccine, 36(5), 660-667. https://doi.org/10.1016/J.VACCINE.2017.12.043

Sherkat, D. E. (2010). Religion and verbal ability. Social Science Research, 39(1), $2-$ 13. https://doi.org/10.1016/J.SSRESEARCH.2009.05.007

Shi, F., Shi, Y., Dokshin, F. A., Evans, J. A., \& Macy, M. W. (2017). Millions of online book co-purchases reveal partisan differences in the consumption of science. Nature Human Behaviour, 1(4), 0079. https://doi.org/10.1038/s41562017-0079

Sibley, C. G., \& Duckitt, J. (2013). The Dual Process Model of Ideology and Prejudice: A longitudinal test during a global recession. The Journal of Social Psychology, 153(4), 448-466. https://doi.org/10.1080/00224545.2012.757544

Sibley, C. G., Harding, J. F., Perry, R., Asbrock, F., \& Duckitt, J. (2010). Personality and prejudice: Extension to the HEXACO personality model. European Journal of Personality, 24(6), 515-534. https://doi.org/10.1002/per.750

Sibley, C. G., Robertson, A., \& Wilson, M. S. (2006). Social Dominance Orientation and Right-Wing Authoritarianism: Additive and Interactive Effects. Political Psychology, 27(5), 755-768. https://doi.org/10.1111/j.1467-9221.2006.00531.x

Sibley, C. G., \& Wilson, M. S. (2007). Political attitudes and the ideology of equality: Differentiating support for liberal and conservative political parties in New Zealand. New Zealand Journal of Psychology, 36(2), 72-84. Retrieved from https://www.psychology.org.nz/wp-content/uploads/NZJP362_SibleyWilson72.pdf

Sidanius, J., \& Pratto, F. (1993). Racism and support of free-market capitalism: A cross-cultural analysis. Political Psychology, 14(3), 381. 
https://doi.org/10.2307/3791704

Sidanius, J., \& Pratto, F. (1999). Social Dominance: An Intergroup Theory of Social Hierarchy and Oppression. Cambridge, UK: Cambridge University Press.

Sidanius, J., Pratto, F., Martin, M., \& Stallworth, L. M. (1991). Consensual racism and career track: Some implications of Social Dominance Theory. Political Psychology, 12(4), 691. https://doi.org/10.2307/3791552

Simis, M. J., Madden, H., Cacciatore, M. A., \& Yeo, S. K. (2016). The lure of rationality: Why does the deficit model persist in science communication? Public Understanding of Science, 25(4), 400-414. https://doi.org/10.1177/0963662516629749

Sjöberg, L. (2004). Principles of risk perception applied to gene technology. EMBO Reports, 5 Spec No(Suppl 1), S47-51. https://doi.org/10.1038/sj.embor.7400258

Slimak, M. W., \& Dietz, T. (2006). Personal values, beliefs, and ecological risk perception. Risk Analysis, 26(6), 1689-1705. https://doi.org/10.1111/j.15396924.2006.00832.x

Sloan, L. (2017). Who tweets in the United Kingdom? Profiling the Twitter population using the British Social Attitudes Survey 2015. Social Media + Society, 3(1), 205630511769898. https://doi.org/10.1177/2056305117698981

Smith, G. A., Alper, B. A., Diamant, J., \& Schiller, A. (2019). Evolution of Pew Research Center's Survey Questions About the Origins and Development of Life on Earth. Retrieved from https://www.pewforum.org/2019/02/06/the-evolutionof-pew-research-centers-survey-questions-about-the-origins-and-developmentof-life-on-earth/

Spence, A., Poortinga, W., Butler, C., \& Pidgeon, N. F. (2011). Perceptions of climate change and willingness to save energy related to flood experience. Nature Climate Change, 1(1), 46-49. https://doi.org/10.1038/nclimate1059

Spence, Alexa, Poortinga, W., \& Pidgeon, N. (2012). The psychological distance of climate change. Risk Analysis, 32(6), 957-972. https://doi.org/10.1111/j.15396924.2011.01695.x

Spence, Alexa, \& Townsend, E. (2006). Examining consumer behavior toward genetically modified (GM) food in Britain. Risk Analysis, 26(3), 657-670. https://doi.org/10.1111/j.1539-6924.2006.00777.x

Spiro, E. S. (2016). Research opportunities at the intersection of social media and survey data. Current Opinion in Psychology, 9, 67-71. https://doi.org/10.1016/J.COPSYC.2015.10.023

Stanley, S. K., \& Wilson, M. S. (2019). Meta-analysing the association between social dominance orientation, authoritarianism, and attitudes on the environment and climate change. Journal of Environmental Psychology, 61, 46-56. https://doi.org/10.1016/J.JENVP.2018.12.002

Stanley, S. K., Wilson, M. S., \& Milfont, T. L. (2017). Exploring short-term longitudinal effects of right-wing authoritarianism and social dominance orientation on environmentalism. Personality and Individual Differences, 108, 
174-177. https://doi.org/10.1016/J.PAID.2016.11.059

Stanley, S. K., Wilson, M. S., Sibley, C. G., \& Milfont, T. L. (2017). Dimensions of social dominance and their associations with environmentalism. Personality and Individual Differences, 107, 228-236. https://doi.org/10.1016/J.PAID.2016.11.051

Statistics New Zealand. (2013). 2013 Census totals by topic. Retrieved from http://archive.stats.govt.nz/Census/2013-census/data-tables/total-by-topic.aspx

Steensland, B., Robinson, L. D., Wilcox, W. B., Park, J. Z., Regnerus, M. D., \& Woodberry, R. D. (2000). The measure of American religion: Toward improving the state of the art. Social Forces, 79(1), 291-318. https://doi.org/10.1093/sf/79.1.291

Stern, P. C., Dietz, T., \& Guagnano, G. A. (1995). The New Ecological Paradigm in social-psychological context. Environment and Behavior, 27(6), 723-743. https://doi.org/10.1177/0013916595276001

Stets, J. E., \& Burke, P. J. (2000). Identity Theory and Social Identity Theory. Social Psychology Quarterly, 63(3), 224. https://doi.org/10.2307/2695870

Stojanov, A., \& Halberstadt, J. (2019). The Conspiracy Mentality Scale. Social Psychology, 50(4), 215-232. https://doi.org/10.1027/1864-9335/a000381

Stroud, N. J. (2014). Selective exposure theories. In K. Kenski \& K. H. Jamieson (Eds.), The Oxford Handbook of Political Communication. https://doi.org/10.1093/oxfordhb/9780199793471.013.009_update_001

Suldovsky, B. (2016). In science communication, why does the idea of the public deficit always return? Exploring key influences. Public Understanding of Science, 25(4), 415-426. https://doi.org/10.1177/0963662516629750

Suldovsky, B. (2017). The Information Deficit Model and climate change communication. In Oxford Research Encyclopedia of Climate Science. https://doi.org/10.1093/acrefore/9780190228620.013.301

Sunstein, C. R. (2016). The Ethics of Influence. https://doi.org/10.1017/CBO9781316493021

Swami, V. (2012). Social psychological origins of conspiracy theories: The case of the Jewish conspiracy theory in Malaysia. Frontiers in Psychology, 3, 280. https://doi.org/10.3389/fpsyg.2012.00280

Swami, V., Coles, R., Stieger, S., Pietschnig, J., Furnham, A., Rehim, S., \& Voracek, M. (2011). Conspiracist ideation in Britain and Austria: Evidence of a monological belief system and associations between individual psychological differences and real-world and fictitious conspiracy theories. British Journal of Psychology, 102(3), 443-463. https://doi.org/10.1111/j.20448295.2010.02004.x

Sylwester, K., \& Purver, M. (2015). Twitter language use reflects psychological differences between democrats and republicans. PLOS ONE, 10(9), e0137422. https://doi.org/10.1371/journal.pone.0137422 
Taber, C. S., \& Lodge, M. (2006). Motivated skepticism in the evaluation of political beliefs. American Journal of Political Science, 50(3), 755-769.

https://doi.org/10.1111/j.1540-5907.2006.00214.x

Tajfel, H., \& Turner, J. C. (1979). An integrative theory of intergroup conflict. In W. G. Austin \& S. Worchel (Eds.), The Social Psychology of Intergroup Relations (pp. 33-47). Monterey, CA: Brooks/Cole.

The challenge of the post-truth era. (2018). Nature Cell Biology, 20(11), 1231-1231. https://doi.org/10.1038/s41556-018-0231-z

Tierney, J., \& Stossel, J. (2018, April). The Left's war on science. City Journal. Retrieved from https://www.city-journal.org/stossel/lefts-war-science

Timpona, J. (2015). Battling misinformation: The scientific consensus as a gateway belief for climate change and GMOs. Retrieved October 17, 2016, from PLOS ECR Blog website: http://blogs.plos.org/thestudentblog/2015/05/15/battlingmisinformation-the-scientific-consensus-as-a-gateway-belief-for-climatechange-and-gmos/

Tranter, B., \& Booth, K. (2015). Scepticism in a changing climate: A cross-national study. Global Environmental Change, 33, 154-164.

https://doi.org/10.1016/j.gloenvcha.2015.05.003

Unsworth, K. L., \& Fielding, K. S. (2014). It's political: How the salience of one's political identity changes climate change beliefs and policy support. Global Environmental Change, 27, 131-137. https://doi.org/10.1016/J.GLOENVCHA.2014.05.002

Uscinski, Joseph E., Douglas, K., \& Lewandowsky, S. (2017). Climate change conspiracy theories. In Oxford research Encyclopedia, Climate Science (Vol. 1). https://doi.org/10.1093/acrefore/9780190228620.013.328

Valente, M. J., \& MacKinnon, D. P. (2017). Communicating the scientific consensus on human-caused climate change is an effective and depolarizing public engagement strategy: Experimental evidence from a large national replication study. Structural Equation Modeling: A Multidisciplinary Journal, 24(3), 428450. https://doi.org/10.1080/10705511.2016.1274657

van der Linden, S. L. (2014). The social-psychological determinants of climate change risk perceptions, intentions and behaviours: a national study. (The London School of Economics and Political Science). Retrieved from http://etheses.lse.ac.uk/896/

van der Linden, S. L. (2015a). A Conceptual Critique of the Cultural Cognition Thesis. Science Communication. https://doi.org/10.1177/1075547015614970

van der Linden, S. L. (2015b). The conspiracy-effect: Exposure to conspiracy theories (about global warming) decreases pro-social behavior and science acceptance. Personality and Individual Differences, 87, 171-173. https://doi.org/10.1016/j.paid.2015.07.045

van der Linden, S. L., Clarke, C. E., \& Maibach, E. W. (2015). Highlighting consensus among medical scientists increases public support for vaccines: 
evidence from a randomized experiment. BMC Public Health, 15(1), 1207. https://doi.org/10.1186/s12889-015-2541-4

van der Linden, S. L., Leiserowitz, A. A., Feinberg, G. D., \& Maibach, E. W. (2014). How to communicate the scientific consensus on climate change: plain facts, pie charts or metaphors? Climatic Change, 126(1-2), 255-262. https://doi.org/10.1007/s10584-014-1190-4

van der Linden, S. L., Leiserowitz, A. A., Feinberg, G. D., \& Maibach, E. W. (2015). The scientific consensus on climate change as a gateway belief: Experimental evidence. PLoS ONE, 10(2). https://doi.org/doi: 10.1371/journal.pone.0118489

van der Linden, S. L., Leiserowitz, A., \& Maibach, E. (2017). Gateway illusion or cultural cognition confusion? Journal of Science Communication, 16(5), A04.

van der Linden, S. L., Leiserowitz, A., \& Maibach, E. (2018a). Perceptions of scientific consensus predict later beliefs about the reality of climate change using cross-lagged panel analysis: A response to Kerr and Wilson (2018). Journal of Environmental Psychology. https://doi.org/10.1016/J.JENVP.2018.10.002

van der Linden, S. L., Leiserowitz, A., \& Maibach, E. (2018b). Scientific agreement can neutralize politicization of facts. Nature Human Behaviour, 2(1), 2-3. https://doi.org/10.1038/s41562-017-0259-2

van der Linden, S. L., Leiserowitz, A., \& Maibach, E. (2019). The gateway belief model: A large-scale replication. Journal of Environmental Psychology, 62, 4958. https://doi.org/10.1016/J.JENVP.2019.01.009

van der Linden, S. L., Leiserowitz, A., \& Maibach, E. W. (2016). Communicating the scientific consensus on human-caused climate change is an effective and depolarizing public engagement strategy: Experimental evidence from a large national replication study. SSRN Electronic Journal.

https://doi.org/10.2139/ssrn.2733956

van der Linden, S. L., Leiserowitz, A., Rosenthal, S., \& Maibach, E. (2017). Inoculating the public against misinformation about climate change. Global Challenges, 1(2), 1-7. https://doi.org/10.1002/gch2.201600008

van der Linden, S. L., \& Lewandowsky, S. (2015). How to combat distrust of science: The surprising power of the psychology of consensus. Scientific American. Retrieved from https://www.scientificamerican.com/article/how-tocombat-distrust-of-science/

Van Eenennaam, A. L., \& Young, A. E. (2014). Prevalence and impacts of genetically engineered feedstuffs on livestock populations. Journal of Animal Science, 92(10), 4255-4278. https://doi.org/10.2527/jas.2014-8124

van Lange, P. A. M., Joireman, J., \& Milinski, M. (2018). Climate change: What psychology can offer in terms of insights and solutions. Current Directions in Psychological Science, 27(4), 269-274. https://doi.org/10.1177/0963721417753945

van Prooijen, J.-W., \& Douglas, K. M. (2018). Belief in conspiracy theories: Basic 
principles of an emerging research domain. European Journal of Social Psychology, 48(7), 897-908. https://doi.org/10.1002/ejsp.2530

Veltri, G. A., \& Atanasova, D. (2017). Climate change on Twitter: Content, media ecology and information sharing behaviour. Public Understanding of Science, 26(6), 721-737. https://doi.org/10.1177/0963662515613702

Walker, L., Baines, P. R., Dimitriu, R., \& Macdonald, E. K. (2017). Antecedents of retweeting in a (political) marketing context. Psychology \& Marketing, 34(3), 275-293. https://doi.org/10.1002/mar.20988

Weaver, K., Garcia, S. M., Schwarz, N., \& Miller, D. T. (2007). Inferring the popularity of an opinion from its familiarity: A repetitive voice can sound like a chorus. Journal of Personality and Social Psychology, 95(5), 821-833. https://doi.org/10.1037/0022-3514.92.5.821

Wessel, L. (2017). Vaccine myths. Science, 356(6336), 368-372. https://doi.org/10.1126/science.356.6336.368

White, G. (2013). Creation or evolution? UMR SayIt Blog. Retrieved March 25, 2019, from http://sayit.co.nz/blog/creation-or-evolution

Whitson, J. A., Kim, J., Wang, C. S., Menon, T., \& Webster, B. D. (2019). Regulatory focus and conspiratorial perceptions: The importance of personal control. Personality and Social Psychology Bulletin, 45(1), 3-15. https://doi.org/10.1177/0146167218775070

Whittingham, N., Boecker, A., \& Grygorczyk, A. (2019). Personality traits, basic individual values and GMO risk perception of twitter users. Journal of Risk Research, 1-19. https://doi.org/10.1080/13669877.2019.1591491

WHO. (2019). Ten threats to global health in 2019. Retrieved March 25, 2019, from https://www.who.int/emergencies/ten-threats-to-global-health-in-2019

Whyman, R. A., Mahoney, E. K., \& Børsting, T. (2016). Community water fluoridation: attitudes and opinions from the New Zealand Oral Health Survey. Australian and New Zealand Journal of Public Health, 40(2), 186-192. https://doi.org/10.1111/1753-6405.12408

Wilson, M. S. (2011). A Descriptive Summary of the Sunday Star Times and Psychology@VUW 'Politics and Rugby'Survey. Retrieved from https://www.victoria.ac.nz/psyc/attachments/wilson-2011-sst-descriptivesummary.pdf

Wilson, M. S. (2015). A dual-process motivational model of climate change belief. Annual Conference of the International Society for Political Psychology, July, 2015. San Diego, United States.

Wilson, M. S., Bulbulia, J., \& Sibley, C. G. (2014). Differences and similarities in religious and paranormal beliefs: A typology of distinct faith signatures.

Religion, Brain \& Behavior, 4(2), 104-126. https://doi.org/10.1080/2153599X.2013.779934

Wilson, M. S., \& Rose, C. (2014). The role of paranoia in a dual-process motivational model of conspiracy belief. In J.-W. van Prooijen \& P. A. M. van 
Lange (Eds.), Power, Politics, and Paranoia (pp. 273-291).

https://doi.org/10.1017/CBO9781139565417.019

Wilson, M. S., \& Sibley, C. G. (2013). Social dominance orientation and right-wing authoritarianism: Additive and interactive effects on political conservatism. Political Psychology, 34(2), 277-284. https://doi.org/10.1111/j.14679221.2012.00929.x

Wilson, N., \& Beaglehole, R. (2014). Options for expanding community water fluoridation in New Zealand. New Zealand Medical Journal, 127(1407), 82-83. Retrieved from https://www.nzma.org.nz/journal/read-the-journal/allissues/2010-2019/2014/vol-127-no-1407/6393

Wilson, N., Edwards, R., Cleghorn, C., Baker, M., Shaw, C., Chapman, R., \& Howden-Chapman, P. (2017, September 14). Climate Change and Recent Actions of NZ Political Parties. Public Health Expert Blog. Retrieved from https://blogs.otago.ac.nz/pubhealthexpert/2017/09/14/climate-change-andrecent-actions-of-nz-political-parties/

Wojcik, S., Huges, A., \& Remy, E. (2019). About one-in-five adult Twitter users in the U.S. follow Trump. Retrieved from https://www.pewresearch.org/facttank/2019/07/15/about-one-in-five-adult-twitter-users-in-the-u-s-follow-trump/

Wojcik, S., Hughes, A., \& Cohn, S. (2019). Sizing Up Twitter Users. Retrieved from https://www.pewinternet.org/2019/04/24/sizing-up-twitter-users/

Wojcik, S. P., Hovasapian, A., Graham, J., Motyl, M., \& Ditto, P. H. (2015). Conservatives report, but liberals display, greater happiness. Science, 345(6227), 1243-1246. https://doi.org/10.1126/science.1260817

Wood, M. J., Douglas, K. M., \& Sutton, R. M. (2012). Dead and alive: Beliefs in contradictory conspiracy theories. Social Psychological and Personality Science, 3(6), 767-773. https://doi.org/10.1177/1948550611434786

Wood, M. J., \& Gray, D. (2019). Right-wing authoritarianism as a predictor of proestablishment versus anti-establishment conspiracy theories. Personality and Individual Differences, 138, 163-166. https://doi.org/10.1016/J.PAID.2018.09.036

Yoon, M., \& Lai, M. H. C. (2018). Testing factorial invariance with unbalanced samples. Structural Equation Modeling: A Multidisciplinary Journal, 25(2), 201-213. https://doi.org/10.1080/10705511.2017.1387859

York, J. C., Greene, D., \& Gebhart, G. (2019). Censorship can't be the only answer to disinformation online. Retrieved August 1, 2019, from Electronic Frontier Foundation website: https://www.eff.org/deeplinks/2019/05/censorship-cant-beonly-answer-disinformation-online

Zaval, L., Keenan, E. A., Johnson, E. J., \& Weber, E. U. (2014). How warm days increase belief in global warming. Nature Climate Change, 4(2), 143-147. https://doi.org/10.1038/nclimate2093

Zhang, C., Wohlhueter, R., \& Zhang, H. (2016). Genetically modified foods: A critical review of their promise and problems. Food Science and Human 
Wellness, 5(3), 116-123. https://doi.org/10.1016/J.FSHW.2016.04.002

Zigerell, L. J. (2012). Science knowledge and biblical literalism. Public Understanding of Science, 21(3), 314-322.

https://doi.org/10.1177/0963662510391723 



\section{Appendices}

\section{Appendix A}

Study 1 measures

Table A.1

Study 1 measures.

\begin{tabular}{lr}
\hline Measure & Items \\
\hline $\begin{array}{l}\text { Pre-/ post-treatment consensus } \\
\text { estimates [0-100 sliding scale] }\end{array}$ & $\begin{array}{r}\text { What percentage of climate scientists do you think agree that climate } \\
\text { change is happening and caused by humans? }\end{array}$ \\
& $\begin{array}{c}\text { What percentage of food scientists do you think agree that food made } \\
\text { from genetically modified plants and animals is safe to eat? }\end{array}$
\end{tabular}

GM Food safety belief [7-point Likert scale]

Climate belief [7-point Likert scale]

Climate concern [7-point scale; not at all concerned - very concerned]

Climate policy support [5-point scale; strongly oppose strongly support]
Please rate your agreement with the following statements

GM foods are safe to eat

GM food can be harmful to public health*

GM ingredients in food can cause illness*

The climate is always changing and what we are currently observing is just natural fluctuation. *

Most of the warming over the last 50 years is due to the increase in greenhouse gas concentrations.

The burning of fossil fuels over the last 50 years has caused serious damage to the planet's climate.

Human CO2 emissions cause climate change.

Humans are too insignificant to have an appreciable impact on global temperature. *

Please indicate your level of concern in response to the questions below

How concerned, if at all, are you about climate change?

Considering any potential effects of climate change which there might be on you personally, how concerned, if at all, are you about climate change?

Considering any potential effects of climate change there might be on society in general, how concerned are you about climate change?

Below are some actions the New Zealand government could take in relation to climate change or GM food. Please let us know how much you would support or oppose these policies.

Lowering GST and increasing tax on petrol by $23 \mathrm{c}$ per litre Providing tax rebates for people who purchase electric vehicles Including farmers in the Emissions Trading Scheme (requiring them to pay for greenhouse gas emissions from livestock and operations)

Subsidising the cost of insulating houses to make them more energy efficient 


\begin{tabular}{lc}
\hline $\begin{array}{l}\text { Anti GM policy [5-point scale; } \\
\text { strongly oppose - strongly } \\
\text { support] }\end{array}$ & $\begin{array}{c}\text { Requiring all food sold in New Zealand containing ingredients from GM } \\
\text { plants and animals to be labelled as such } \\
\text { Removing restrictions on growing GM crops in New Zealand* } \\
\text { Banning the sale of food containing GM ingredients in New Zealand } \\
\text { Requiring meat and other products from animals fed GM crops to be } \\
\text { labelled as such }\end{array}$ \\
$\begin{array}{l}\text { Climate intentions [7-point } \\
\text { Likert scale] }\end{array}$ & $\begin{array}{l}\text { following statements } \\
\text { I intend to help reduce climate change by changing my behaviour }\end{array}$ \\
& $\begin{array}{l}\text { I intend to do my bit to help tackle climate change } \\
\text { I intend to address climate change by taking personal action }\end{array}$ \\
GM intentions [7-point Likert & $\begin{array}{l}\text { I plan to never eat genetically modified food } \\
\text { scale] }\end{array}$ \\
I will only buy 'GM-free' food products
\end{tabular}

*reverse scored item. 


\section{Appendix B}

\section{Study 1: Effects of consensus messages on concern, intentions and policy support}

The Gateway Belief Model also posits that increases in belief that human-caused climate change is occurring have a 'cascading' effect on related attitudes and beliefs, increasing concern about climate change and support for public action. Study 1 included several attitudinal measures to investigate the wider effects of consensus messages. While not the focus of the study, for completeness I report in this appendix these measures and compare results between experimental groups. A full list of all survey items can be found in Appendix A.

\section{Measures}

Climate concern: A 3-item scale from Spence, Poortinga and Pidgeon (2012) was used to measure concern regarding the effects of climate change was measured using. These items covered the three main types of concern associated with climate change: general concern, personal concern and societal concern (example: Considering any potential effects of climate change there might be on society in general, how concerned are you about climate change?). Participants responded on a scale ranging from 1 (Not at all concerned) to 7 (Very concerned $)$. The scale displayed good reliability $(\alpha=.88)$, similar to that reported in Spence et al. $(\alpha=.83)$.

Climate intentions: Personal intentions to act to address climate change were measured using a 3-item scale developed by van der Linden (van der Linden, 2014). Rather than focusing on specific actions, this scale captures an overall general intent (example: I intend to do my bit to help tackle climate change). Participants indicated their agreement with the statements on a Likert scale ranging from 1 (Strongly disagree) to 7 (Strongly agree). The scale displayed excellent reliability $(\alpha=.94)$, higher than that reported by van der Linden $(\alpha$ $=.85)$,

Climate policy support: An ad hoc policy support scale was constructed to measure participants' support for climate mitigation policy options. The design was modelled on previous scales (e.g., Dietz, Dan, \& Shwom, 2007; Leiserowitz, 2006) but incorporated four real-world, New Zealand-specific climate change mitigation policy options drawn from media reports and policy documents (Parliamentary Commissioner for the Environment, 2016; Polkinghorne, 2016; Royal Society of New Zealand, 2016). For these items and the 
GM policy items detailed below, participants were provided with the following instructions: Below are some actions the New Zealand government could take in relation to climate change or GM food. Please let us know how much you would support or oppose these policies (example item: Providing tax rebates for people who purchase electric vehicles). Participants indicated their level of support for each policy on a 5-point scale ranging from 1 (strongly oppose) to 5 (strongly support). The reliability of this scale was poor $(\alpha=.56)$, however Cronbach's $\alpha$ tends to be artificially deflated when there are only a small number of items in a scale. The mean inter-item correlation was .25 and the corrected item-total correlations for all items were above .30 which, as a rule of thumb, indicates that the items are measuring the same construct and could be usefully retained (Field, 2009).

GM intentions: Participants' personal intentions regarding GM food (eating, purchasing and checking labels) were measured using a 4-item scale adapted from Spence and Townsend (2006). Two additional items were added to the original 2-item scale to improve reliability (example item: I intend to never buy food containing GM ingredients). Responses were captured on a 7-point Likert scale. The scale displayed good reliability $(\alpha=$ $.86)$.

GM policy support: As with the climate policy support scale, GM policy preferences were measured using an ad hoc scale comprised of four New Zealand policy options sourced from the media and policy documents (Galloway, 2016; GE Free NZ, 2016; NZ Parliament, 2015; Peters \& Heinemann, 2012). Most relevant policy discussion relating to GM food safety has focused on restrictions around GM food availability and labelling. As such, the wording of items in this scale means that higher values indicate greater support for restrictions and regulations for GM food (example: Banning the sale of food containing GM ingredients in New Zealand). Participants indicated their level of support for each policy on a 5-point scale ranging from 1 (strongly oppose) to 5 (strongly support). The scale displayed marginal acceptability $(\alpha=.68)$. All corrected item-total correlations were greater than .30 and the mean inter-item correlation was .35.

\section{Results}

A multivariate ANOVA found no significant differences between high and low consensus message and control conditions across all dependent climate variables, $F(8,818)$ $=.871, p=.54$; Wilk's $\Lambda=0.983, \eta_{\mathrm{p}}{ }^{2}=.01$ (Table $\left.\mathrm{B}\right)$. That is, participants who read a message about high or low consensus regarding the reality of human-caused climate change 
did not significantly differ from the control group in terms of: belief in the reality of humancaused climate change, concern over climate change, intentions to personally act to mitigate climate change or support for climate change policies. This finding doesn't support the Gateway Belief Model, which posits that interventions which change perceptions of consensus should also change personal beliefs about climate change.

Table B.1

Descriptive statistics for climate-related outcomes after reading a high or low climate consensus message or no message.

\begin{tabular}{lcccccccc}
\hline & Climate belief & \multicolumn{2}{c}{ Concern } & \multicolumn{2}{c}{ Policy support } & \multicolumn{2}{c}{ Intentions } \\
& $M$ & $S D$ & $M$ & $S D$ & $M$ & $S D$ & $M$ & $S D$ \\
\hline $\begin{array}{l}\text { High (97\%) climate } \\
\text { message }\end{array}$ & 5.47 & $(0.88)$ & 5.78 & $(1.18)$ & 3.64 & $(0.62)$ & 5.09 & $(1.05)$ \\
$\begin{array}{l}\text { Low (63\%) climate } \\
\text { message }\end{array}$ & & & & & & & & \\
Control & 5.44 & $(0.80)$ & 5.65 & $(1.06)$ & 3.54 & $(0.58)$ & 4.97 & $(1.05)$ \\
& 5.40 & $(0.87)$ & 5.58 & $(1.10)$ & 3.49 & $(0.57)$ & 4.84 & $(1.20)$ \\
\hline
\end{tabular}

A MANOVA examining GM food safety beliefs, intentions and policy preferences indicated a significant difference between the high GM consensus message and control conditions, $F(3,249)=3.12, p=.03$; Hotelling's $T^{2}=5.73, \eta_{\mathrm{p}}{ }^{2}=.04$ (Table B). Independent samples t-tests revealed significant differences between the message and control conditions for GM food safety beliefs, $t(251)=-2.31, p=.02, d=0.29$, and anti-GM policy support $t(251)=0.54, p=.008, d=0.34$. These results indicate that exposure to a message about a high level of scientific agreement regarding the safety of GM food increases individuals' reported belief that GM food is safe and lowers their support for policies regulating GM food products. These findings offer support for the Gateway Belief Model which predicts that changes in perceptions of scientific consensus are causally related to changes in personal beliefs.

Table B.2

Descriptive statistics for GM-related outcomes after reading a high GM consensus message or no message.

\begin{tabular}{lcccccc}
\hline & \multicolumn{2}{c}{ GM food safety } & \multicolumn{2}{c}{ GM intentions } & \multicolumn{2}{c}{ Anti GM-policy } \\
& $M$ & $S D$ & $M$ & $S D$ & $M$ & $S D$ \\
\hline High $(97 \%) \mathrm{GM}$ & & & & & & \\
message $^{\mathrm{a}}$ & $4.37^{\mathrm{b}}$ & $(1.21)$ & 3.02 & $(1.24)$ & $3.31^{\mathrm{b}}$ & $(0.64)$ \\
Control $^{\mathrm{b}}$ & $4.02^{\mathrm{a}}$ & $(1.23)$ & 3.11 & $(1.25)$ & $3.51^{\mathrm{a}}$ & $(0.55)$ \\
\hline
\end{tabular}

Superscripts denote significant difference at $p<.05$.

\section{Discussion}


The current study found that consensus messages had no overall effect on concern over climate change, personal intentions to act or support for climate mitigation policies, as seen in previous studies examining similar constructs (Bolsen \& Druckman, 2018a; Deryugina \& Shurchkov, 2016; van der Linden et al., 2014). Exceptions include an experiment by Bolsen et al. (2014) which found that while a high consensus message did not influence belief in human-caused climate change, it did increase support for climate policies and willingness to take personal action. A large $(N=3,150)$ survey experiment undertaken by van der Linden et al. (2017) also found that a single consensus statement led to significant within-subjects increases in worry about climate change and general support for action. Taken in the context of this previous research, the current results suggest that any immediate effects of consensus messaging on more distal beliefs related to climate change, such as personal intentions and policy support, are at best small and difficult to detect.

This is the first study to date to examine the effects of consensus messages on personal intentions to buy or consume GM food and GM policy preferences. The results imply that actors seeking to decrease support for GM regulation would find consensus messaging a useful tactic in shifting public opinion. 


\section{Appendix C}

\section{Study 1 mediation models}

Tables C.1-3 outline the regression models used to determine the beta values for the paths in Figures 4.1-4.3 in the main text.

The first model in each table represents the effect of reading a high consensus message on climate beliefs ( $c$ '; the total effect). The second model shows the effect of the message condition on perceptions of consensus $(a)$ and the final model shows the effect of both perceptions of consensus and message condition on climate beliefs ( $b$ and c respectively). These analyses include pre-treatment perceptions of consensus as a covariate in all paths, thus interpreting the mediator as change in perceived consensus (Valente \& MacKinnon, 2017).

Table C.1

Effect of $97 \%$ climate consensus message on climate beliefs mediated via perceptions of consensus, controlling for pre-treatment estimates of consensus

\begin{tabular}{|c|c|c|c|c|c|c|c|c|c|}
\hline & \multicolumn{9}{|c|}{ Outcome } \\
\hline & \multicolumn{3}{|c|}{ Climate change belief } & \multicolumn{3}{|c|}{$\begin{array}{l}\text { Post-treatment consensus } \\
\text { estimate }\end{array}$} & \multicolumn{3}{|c|}{ Climate change belief } \\
\hline & $B$ & $\mathrm{SE}$ & $\beta$ & $B$ & $\mathrm{SE}$ & $\beta$ & $B$ & SE & $\beta$ \\
\hline Constant & $4.03 * * *$ & 0.28 & & $44.86 * * *$ & 4.19 & & 3.46 & 0.33 & \\
\hline Message (vs. control) & 0.08 & 0.10 & 0.05 & $12.29 * * *$ & 1.57 & $0.39 * * *$ & -0.08 & 0.11 & -0.04 \\
\hline $\begin{array}{l}\text { Pre-treatment consensus } \\
\text { estimate }\end{array}$ & $0.02 * * *$ & 0.00 & $0.30 * * *$ & $0.44 * * *$ & 0.05 & $0.44 * * *$ & $.01 * *$ & 0.00 & $0.20 * *$ \\
\hline $\begin{array}{l}\text { Post-treatment consensus } \\
\text { estimate }\end{array}$ & & & & & & & $.01 * *$ & 0.00 & $0.23 * *$ \\
\hline$R^{2}$ & .09 & & & .34 & & & .13 & & \\
\hline
\end{tabular}

Unstandardized and standardized regression coefficients shown.

$* * p>.01 * * * p<.001$. 
Table C. 2

Effect of $63 \%$ climate consensus message on climate beliefs mediated via perceptions of consensus, controlling for pre-treatment estimates of consensus

\section{Outcome}

\begin{tabular}{|c|c|c|c|c|c|c|c|c|c|}
\hline & \multicolumn{3}{|c|}{ Climate change belief } & \multicolumn{3}{|c|}{$\begin{array}{l}\text { Post treatment consensus } \\
\text { estimate }\end{array}$} & \multicolumn{3}{|c|}{ Climate change belief } \\
\hline & $B$ & SE & $\beta$ & $B$ & SE & $\beta$ & $B$ & SE & $\beta$ \\
\hline Constant & $3.89 * * *$ & 0.24 & & $42.42 * * *$ & 3.69 & & $3.59 * * *$ & 0.29 & \\
\hline Message (vs. control) & 0.06 & 0.09 & 0.04 & $-12.34 * * *$ & 1.46 & $-0.39 * * *$ & 0.15 & 0.11 & 0.09 \\
\hline $\begin{array}{l}\text { Pre-treatment consensus } \\
\text { estimate }\end{array}$ & $0.02 * * *$ & 0.00 & $0.37 * * *$ & $0.47 * * *$ & 0.04 & $0.50 * * *$ & $0.02 * * *$ & 0.00 & $0.31 * * *$ \\
\hline $\begin{array}{l}\text { Post-treatment consensus } \\
\text { estimate }\end{array}$ & & & & & & & $0.01 \dagger$ & 0.00 & $0.13 \dagger$ \\
\hline$R^{2}$ & .14 & & & .41 & & & .15 & & \\
\hline
\end{tabular}

Unstandardized and standardized regression coefficients shown.

$\dagger p<.10, * * * p<001$

Table C.3

Effect of 97\% GM consensus message on climate beliefs mediated via perceptions of consensus, controlling for pre-treatment estimates of consensus

\section{Outcome}

\begin{tabular}{|c|c|c|c|c|c|c|c|c|c|}
\hline & \multicolumn{3}{|c|}{ GM food safety beliefs } & \multicolumn{3}{|c|}{$\begin{array}{l}\text { Post-treatment consensus } \\
\text { estimate }\end{array}$} & \multicolumn{3}{|c|}{ GM food safety beliefs } \\
\hline & $B$ & SE & $\beta$ & $B$ & SE & $\beta$ & $B$ & SE & $\beta$ \\
\hline Constant & $2.63 * * *$ & 0.20 & & $31.01 * * *$ & 3.33 & & $2.23 * * *$ & 0.22 & \\
\hline Message (vs. control) & $0.36 * *$ & 0.14 & $0.15 * *$ & $29.90 * * *$ & 2.32 & $0.58 * * *$ & -0.02 & 0.17 & -0.01 \\
\hline $\begin{array}{l}\text { Pre-treatment consensus } \\
\text { estimate }\end{array}$ & $0.02 * * *$ & 0.00 & $0.45 * * *$ & $.46 * * *$ & 0.05 & $0.41 * * *$ & $0.02 * * *$ & 0.00 & $0.34 * * *$ \\
\hline $\begin{array}{l}\text { Post-treatment consensus } \\
\text { estimate }\end{array}$ & & & & & & & $0.01 * * *$ & 0.00 & $0.27 * * *$ \\
\hline$R^{2}$ & .22 & & & .50 & & & .26 & & \\
\hline
\end{tabular}

Unstandardized and standardized regression coefficients shown.

$* * p>.01, * * * p<.001$.

\section{Incorporating low consensus GM message into control group for GM analyses.}

The 'low consensus' ('63\%') GM message used in this study was in fact close to the extant level of perceived consensus in our sample and did not significantly affect consensus estimates. As such we ran supplementary analyses including this group in the control condition to increase statistical power. The results of the GM analyses were not substantively changed (Table C.4). 
Table C.4

Effect of 97\% GM consensus message on GM food safety beliefs mediated via perceptions of consensus (low GM consensus and no message conditions combined as control group), controlling for pre-treatment estimates of consensus.

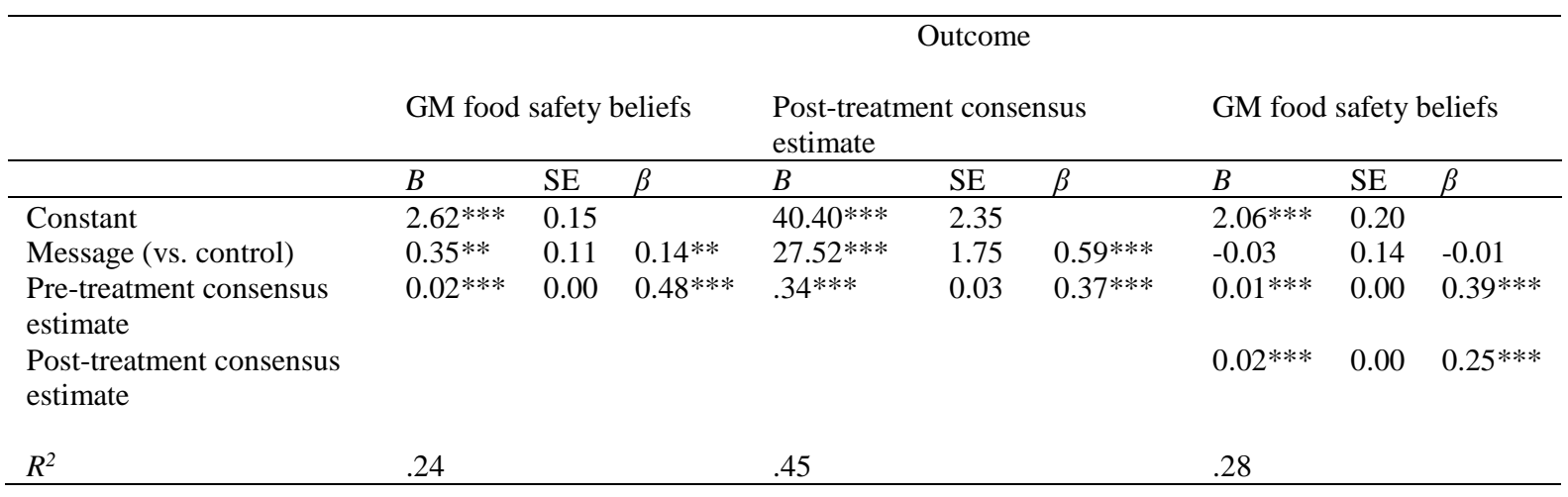

Unstandardized and standardized regression coefficients shown. $* * p>.01, * * * p<.001$.

In this model we report a significant indirect effect, $a b=0.38,95 \% \mathrm{CI}[0.21,0.57]$; $a b_{p s}=0.35,95 \% \mathrm{CI}[.20, .52]$. This is comparable to the results reported in the main text comparing the $97 \%$ GM message group to the control group only ( $a b=0.39,95 \%$ CI $[0.18$, $\left.0.62] ; a b_{p s}=0.35,95 \% \mathrm{CI}[.17, .55]\right)$, with slight reductions in the size of the confidence intervals. 



\section{Appendix D}

\section{Study 2 Supplementary material}

This appendix presents the measures used in Study 2 (Table D.1), their descriptive statistics and intercorrelations (Table D.2), and supplementary analyses comparing crosslagged effects between participants with liberal or conservative voting intentions.

Table D.1

Study 2 measures

\begin{tabular}{ll}
\hline Measure & Items \\
\hline Belief in anthropogenic & Burning of fossil fuels on the scale observed over the last 50 \\
climate change scale (7-point & years has increased atmospheric temperature to an \\
Likert scale; Strongly & appreciable degree. \\
disagree - Strongly agree) & Burning fossil fuels on the scale observed over the last 50 \\
& years will cause serious negative changes to the planet's \\
& climate unless there is a substantial switch to non CO2- \\
& emitting energy sources. \\
& Burning fossil fuels increases atmospheric temperature to \\
& some measurable degree. \\
Burning fossil fuels on the scale observed over the last 50 & years has caused serious negative changes to the planet's \\
climate.
\end{tabular}


Table D.2

Descriptive statistics and correlation matrix for all variables in cross-lagged model

\begin{tabular}{lcccccc}
\hline & Mean & SD & 1. & 2. & 3. & 4. \\
\hline 1. ACC belief T1 & 5.16 & 1.05 & & & & \\
2. Climate consensus T1 & 77.98 & 18.42 & 0.31 & & & \\
3. ACC Belief T2 & 5.18 & 1.16 & 0.59 & 0.30 & \\
4. Climate consensus T2 & 78.73 & 19.84 & 0.29 & 0.44 & 0.33
\end{tabular}

Note. Pearson's' $r$ correlations. ACC Belief $=$ belief in anthropogenic climate change. Consensus variables represent the estimated number of climate scientists out of 100 that believe anthropogenic climate change is occurring.

All correlations significant at $p<.001$

\section{Moderation of cross-lagged effects by political orientation.}

We conducted further analyses to explore the possibility that political orientation moderates the effects shown in the cross-lagged model presented in the main text (Figure 4.6). Acceptance of climate science is moderately correlated with political ideology in New Zealand, with conservatives less likely to endorse the reality of anthropogenic climate change (Milfont et al., 2015).

We used voting preference as an indicator of political orientation. In the survey, participants were asked the open-ended question "If an election were held today, which political party would you cast your party vote for?" Participants who indicated they would vote for the Labour or Green parties were coded as 'Liberal' $(N=129)$ and participants who indicated they would vote for the National party were coded 'conservative' $(N=119)$. For further detail on New Zealand political voting preferences and their association with political ideology, see Greaves, Osborne and Sibley (2015). The remainder of participants (those who gave no response, did not know for which party they would vote or indicated support for less clearly categorised minor parties; $N=108$ ) were excluded from the analysis.

Descriptive statistics and zero-order correlations for each group are shown in Tables D.3 and D.4. Independent samples t-tests revealed that participants in the liberal group scored higher on the climate change beliefs scale than those in the conservative group at both Time 1 $\left(M_{L i b}=5.37, S D=.97 ; M_{C o n}=4.91, S D=0.92 ; p<.001\right)$ and Time $2\left(M_{L i b}=5.40, S D=\right.$ $\left.1.14 ; M_{C o n}=5.00, S D 1.13 ; p<.01\right)$. There were no significant differences between groups 
for estimates of consensus at Time $1\left(M_{L i b}=79.43 \%, S D=20.03 ; M_{C o n}=76.31 \%, S D=\right.$ $15.76 ; p=.18)$ or Time $2\left(M_{\text {Lib }}=77.87 \%, S D=22.55 ; M_{C o n}=78.06 \%, S D=18.08 ; p=.94\right)$.

Table D.3

Descriptive statistics and correlation matrix for participants with liberal voting intentions $(N$ $=129$.

$\begin{array}{lllll}\text { Mean } & \text { SD } & 1 . & 2 . & 3 .\end{array}$

\begin{tabular}{lccllll}
\hline 1. ACC Belief T1 & 5.37 & 0.97 & 1 & & \\
2. Climate consensus T1 & 79.43 & 20.037 & $0.28^{* * *}$ & 1 & & \\
3. ACC Belief T2 & 5.40 & 1.14 & $0.59^{* * * *}$ & $0.27^{* *}$ & 1 & \\
4.Climate consensus T2 & 77.87 & 22.55 & $0.37^{* * *}$ & $0.56^{* * *}$ & $0.46^{* * *}$ & 1
\end{tabular}

Pearson's' $r$ correlations. ${ }^{* *} p<.01, * * * p<.001$. ACC Belief $=$ belief in anthropogenic climate change. Consensus variables represent the estimated number of climate scientists out of 100 that believe anthropogenic climate change is occurring.

Table D.4

Descriptive statistics and correlation matrix for participants with conservative voting intentions $(N=119)$.

\begin{tabular}{lllllll}
\hline & Mean & SD & 1. & 2. & 3. & 4. \\
\hline 1. ACC Belief T1 & 4.92 & 0.92 & 1 & & & \\
2. Climate consensus T1 & 76.31 & 15.76 & $0.25^{* *}$ & 1 & & \\
3. ACC Belief T2 & 5.00 & 1.13 & $0.50^{* * *}$ & $0.21^{*}$ & 1 & \\
4. Climate consensus T2 & 78.06 & 18.06 & 0.09 & $0.28^{* *}$ & 0.13 & 1
\end{tabular}

Pearson's' $r$ correlations. $* * p<.05, * * p<.01$, *** $p<.001$. ACC Belief $=$ belief in anthropogenic climate change. Consensus variables represent the estimated number of climate scientists out of 100 that believe anthropogenic climate change is occurring.

To examine differences between the liberal and conservative groups, we conducted invariance testing on the key parameters (auto-regressive and cross-lagged paths) outlined the main model (Figure 4.6 in the main text) using nested models in the $R$-based lavaan package (Rosseel, 2012). The initial model allowed all parameters to vary freely between groups $\left(\chi^{2}(54)=107.62\right)$. Subsequent models constrained one of the paths to be equal between 
groups while all other parameters were allowed to vary freely. If model fit was significantly reduced by constraining a given path it was determined that the path differed significantly between groups (Jose, 2013).

The beta weights of the auto-regressive and cross-lagged paths in the unconstrained model are shown in Table D.5. The table also shows the model fit when each path is constrained to be equal between groups. Notably, for both liberal and conservative groups, Time 2 perceptions of consensus did not prospectively predict Time 2 beliefs about climate change. Constraining this path to be equal between groups did not significantly alter model fit. This indicates that for both liberal and conservative voters, perceptions of scientific consensus did not influence later belief in the reality of anthropogenic climate change.

For participants with liberal voting intentions, perception of agreement among scientists prospectively predicts later personal agreement that humans are changing the climate. However, for participants with conservative voting intentions, there is no significant crosslagged effect. Constraining the effect of Time 1 perceptions of consensus on Time 2 beliefs significantly reduced model fit - indicating that the paths are significantly different between liberal and conservative groups.

Table D.5

Unconstrained beta weights for auto-regressive and cross-lagged paths and model fit when parameter constrained.

\begin{tabular}{lllc}
\hline Parameter & Liberal & $\begin{array}{l}\beta \\
\text { Conservative }\end{array}$ & $\begin{array}{c}\text { Model fit }\left(\chi^{2}(55)\right) \\
\text { when parameter } \\
\text { constrained }\end{array}$ \\
\hline T1 Belief $\rightarrow$ T2 Belief & $.67 * * *$ & $.61^{* * *}$ & 109.38 \\
T1 Consensus $\rightarrow$ T2 Belief & $.28^{* * *}$ & .05 & $\mathbf{1 1 3 . 4 5}$ \\
T1 Belief $\rightarrow$ T2 Consensus & .06 & .07 & 107.67 \\
T1 Consensus $\rightarrow$ T2 Consensus & $.47 * * *$ & $.27 * *$ & 110.23 \\
\hline
\end{tabular}

** $p<.01, * * * p<.001$. Bold text indicates a significant difference $(\mathrm{p}<.05)$ between constrained and
unconstrained models.

While the discussion in the main text focused on a lack of support for the gateway belief model - that perceptions of consensus don't predict later beliefs - we offer here a brief 
discussion of the findings regarding the inverse path: beliefs predicting later perceptions of consensus.

Why would individuals' personal certainty about ACC predict their later perceptions of scientific consensus? When people hold attitudes that are mutually inconsistent they experience cognitive dissonance that they may resolve by rationalising one or other position (Festinger, 1957). When a personal belief (the climate is not changing) is inconsistent with an alternative (most scientists believe the climate is changing) then, consistent with the gateway belief model, the personal belief may be revised to increase consistency with the perceived consensus. Alternatively - and as suggested by the current results - individuals may revise their perceptions of the consensus, rather than their own views, to ensure coherence between their beliefs. Related to cognitive dissonance, the overlapping frameworks of motivated reasoning (Kunda, 1990) and selective exposure (Hart et al., 2009) offer further explanation. Individuals are more likely to seek out, be exposed to, attend to and recall information which supports their prior beliefs and attitudes (Taber \& Lodge, 2006). Individuals are also more likely to ascribe expertise to researchers whose views align with their own, which can lead to a biased perception of the scientific consensus (Kahan et al., 2011).

For example, in the case of climate change beliefs, the liberal voting participants in our study were more likely to believe the ACC is occurring and may have selectively consumed media and information which aligned with this view and emphasised the scientific consensus, leading to higher estimates of the scientific consensus at Time 2. There is, to our knowledge, no research directly investigating the extent of self-selected exposure among political participants in New Zealand on the issue of climate change. However, research in other western countries has noted partisan differences in media use, with liberals more likely to consume media endorsing the mainstream scientific position on anthropogenic climate change (L. Feldman, Myers, Hmielowski, \& Leiserowitz, 2014; Kim, 2011). In the case of conservative voting participants in our study, we did not see a similar cross-lagged effect. Content analyses of New Zealand news media indicate that sceptical or denialist viewpoints on ACC receive less coverage than in other national contexts (Chetty, Devadas, \& Fleming, 2015). This may have limited opportunity for selective exposure and/or biased assimilation of information refuting ACC. The current study was not designed to explore the drivers of the political differences reported, so the reasoning above remains speculation until further research is undertaken. 
Another possible explanation for the partisan differences identified in our analysis is that liberal individuals are more inclined to ensure their personal beliefs and what they perceive the scientific community to believe are coherent. The model above suggests that they ensure this coherence by adjusting their perceptions of consensus to match their personal certainty regarding the reality of ACC (rather than vice versa, as proposed by the gateway belief model). Relatively conservative individuals, on the other hand, may be less inclined to ensure coherence between their personal beliefs and perceptions of consensus. Supporting this view, previous US research has shown conservatives are less likely to trust the scientific community (Gauchat, 2012; Hartman et al., 2017) and more likely to disagree with an explicitly acknowledged scientific consensus among scientists regarding ACC (Pasek, 2017). Further research incorporating measures of trust in scientists or perceived expertise of scientists as a moderator of the cross-lagged relationship between beliefs and perceived consensus could potentially explain the partisan differences found in our results. However, to reiterate the key finding presented in the main text, a cross-lagged effect of perceived consensus on later personal belief was not present in either the conservative or liberal voting groups.

It is important to note that the moderation by political orientation did not indicate that political conservatives' relative lack of endorsement of climate change prospectively predicted lower estimates of consensus - but rather that their belief was prospectively unrelated to consensus beliefs. Perhaps scientific consensus on climate change is more important for liberals, because liberals see science as a relevant authority in the matter. Research suggests that conservatives may be less trusting than liberals of scientific authorities, in general, and this extends to scientific authority concerning the climate and environment (e.g. Brewer \& Ley, 2013). Additionally, research not only suggests that conservatives are less likely to seek out scientific sources for guidance about science, but they may be more likely to seek out information from religious authorities regarding those same questions (even after controlling for religiosity: Scheitle, Johnson, \& Ecklund, 2018). Why attend to scientific consensus on a particular issue if there is a preferred, subjectively more credible source? This suggests that were we to inquire about perceptions of religious consensus, we might find the reverse moderation - conservatives' beliefs about climate change (or any other issue upon which religious authorities might take a position) would prospectively predict their consensus beliefs, while the same would not be true for liberals. 
Our analysis did find a difference in climate change endorsement between liberal and conservative voting participants, but no significant difference in perceptions of consensus. We note that this divide is likely smaller than that which would be expected in US samples. A recent analysis of international survey data found that although New Zealand ranked relatively high in terms of climate scepticism $\left(5^{\text {th }}\right.$ out of the 24 nations included in the study), the correlation between political ideology and ACC belief was lower than that seen in the USA (Hornsey et al., 2018a). Over the last decade, conservative political parties in New Zealand have shifted their stance from scepticism to debating the merits of intensive climate mitigation policies (N. Wilson et al., 2017). Thus, the existence anthropogenic climate change - while still treated with scepticism by a sizable minority of New Zealanders appears to no longer be a debated issue in political discourse in New Zealand. 



\section{Appendix E}

\section{Study 3 Party-level analysis}

This appendix details a further analysis from Study 3, comparing the proportion of sceptic followers among followers of different political parties and their MPs. The percentages of followers of NZ political parties who also follow science sceptic accounts are reported in Table E.1 and Figure E.1.

Table E.1

Overlap between followers of science sceptic accounts and followers of specific political parties and their MPs (excluding users who follow multiple parties).

\begin{tabular}{lllrrr}
\hline \multirow{2}{*}{ Party } & \multirow{2}{*}{$\begin{array}{c}\text { Total } \\
\text { accounts in } \\
\text { list }\end{array}$} & $\begin{array}{c}\text { Unique } \\
\text { followers }\end{array}$ & $\begin{array}{r}\text { Climate } \\
\text { sceptic } \\
\text { accounts }\end{array}$ & $\begin{array}{r}\text { Vaccine } \\
\text { sceptic } \\
\text { accounts }\end{array}$ & $\begin{array}{r}\text { GM sceptic } \\
\text { accounts }\end{array}$ \\
\hline Greens & 9 & 28,355 & $1.37 \%$ & $0.98 \%$ & $4.13 \%$ \\
Labour & 43 & 419,169 & $0.45 \%$ & $0.28 \%$ & $0.70 \%$ \\
National & 46 & 39,141 & $2.93 \%$ & $2.92 \%$ & $2.51 \%$ \\
NZ First & 9 & 4,619 & $2.15 \%$ & $0.81 \%$ & $0.60 \%$ \\
ACT & 2 & 1,632 & $8.40 \%$ & $1.51 \%$ & $1.10 \%$ \\
\hline
\end{tabular}




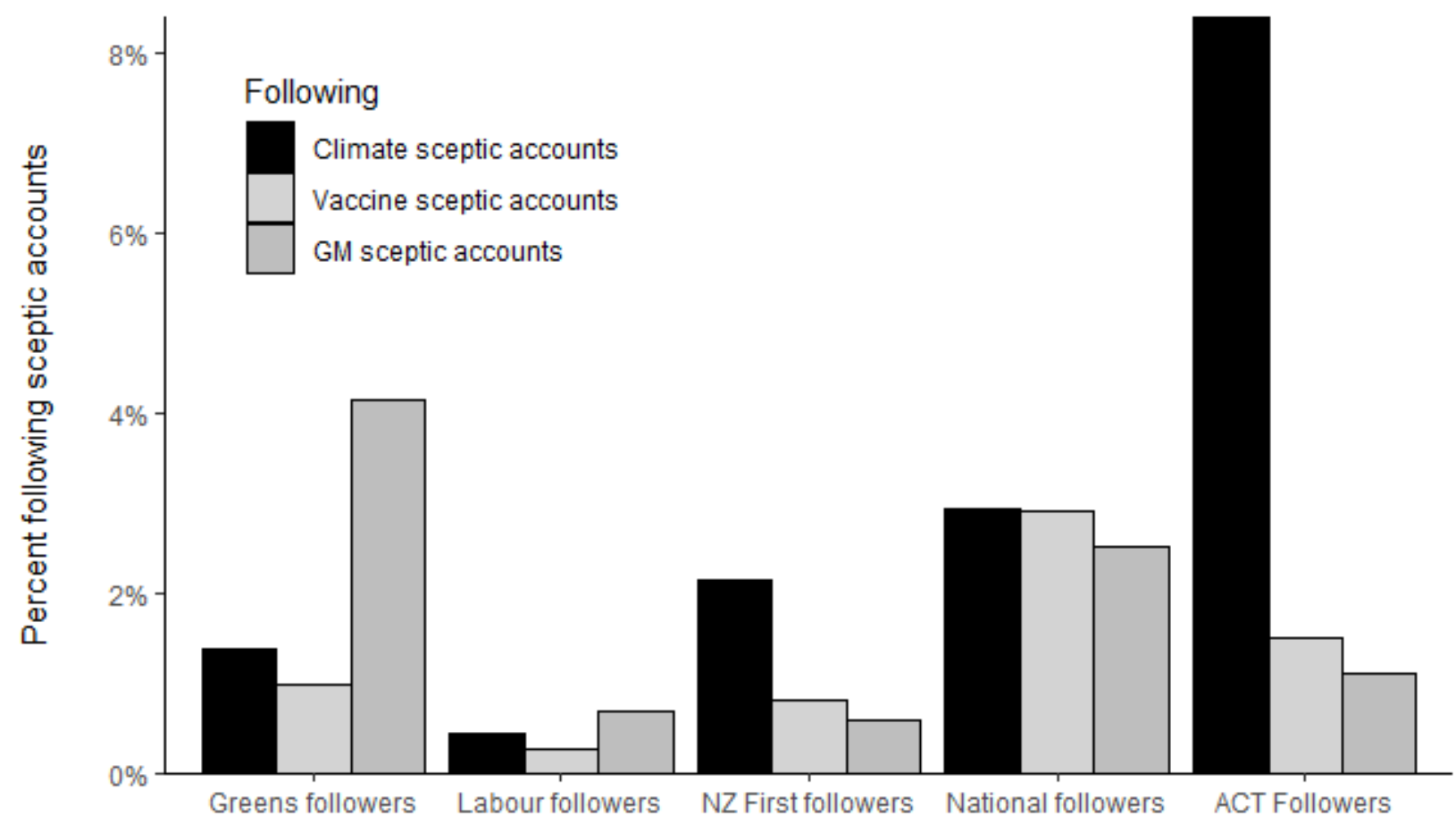

Figure E.1. Percentage of NZ political party Twitter account followers who also follow science sceptic accounts.

Chi-squared tests of independence indicated that party following was related to the following of science sceptic accounts for climate change $\left(\chi^{2}(4)=4022.2, p<.001\right)$, vaccines $\left(\chi^{2}(4)=4044.2, p<.001\right)$ and GM $\left(\chi^{2}(4)=3293.8, p<.001\right)$. Post hoc comparisons indicated that most parties differed significantly from each other (see Tables E.2-4).

Table E.2

Post hoc comparisons of the proportion of climate sceptic followers between parties.

\begin{tabular}{lcccc}
\hline & Greens & National & NZ First & ACT \\
\hline Labour & $363.99 * * *$ & $2781.20^{* * *}$ & $271.82^{* * *}$ & $1913.40^{* * *}$ \\
Greens & & $141.19^{* * *}$ & $14.76^{* *}$ & $378.27 * * *$ \\
National & & & $8.38^{*}$ & $132.94 * * *$ \\
NZ First & & & & $119.11^{* * *}$ \\
\hline
\end{tabular}

$\chi^{2}(1)$ values and significance (with Bonferroni correction).

$* p<.05, * * p<.01, * p<.001$. 
Table E.3

Post hoc comparisons of the proportion of vaccine sceptic followers between parties.

\begin{tabular}{lcccc}
\hline & Greens & National & NZ First & ACT \\
\hline Labour & $326.47^{* * *}$ & $4155.40^{* * *}$ & $40.61^{* * *}$ & $73.02^{* * *}$ \\
Greens & & $234.62^{* * *}$ & $1.06^{* * *}$ & 3.33 \\
National & & & $66.60^{* * *}$ & $9.33^{*}$ \\
NZ First & & & & 4.98 \\
\hline
\end{tabular}

$\chi^{2}(1)$ values with Bonferroni correction.

$*_{p}<.05, * * p<.01, * p<.001$.

Table E.4

Post hoc comparisons of the proportion of GM sceptic followers between parties.

\begin{tabular}{lcccc}
\hline & Greens & National & NZ First & ACT \\
\hline Labour & $2837.50^{* * *}$ & $1107.30^{* * *}$ & 0.48 & 2.75 \\
Greens & & $109.95^{* * *}$ & $134.81^{* * *}$ & $32.30^{* * *}$ \\
National & & & $62.96^{* * *}$ & $10.91^{* *}$ \\
NZ First & & & & 3.12 \\
\hline
\end{tabular}

$\chi^{2}(1)$ values with Bonferroni correction.

$* p<.05, * * p<.01, * p<.001$. 



\section{Appendix F}

\section{Study 3 Details of science sceptic accounts}

Tables F.1-3 detail the accounts included in the final lists of climate, GM and vaccine sceptic accounts respectively.

\section{Table F.1}

Climate sceptic accounts and descriptions

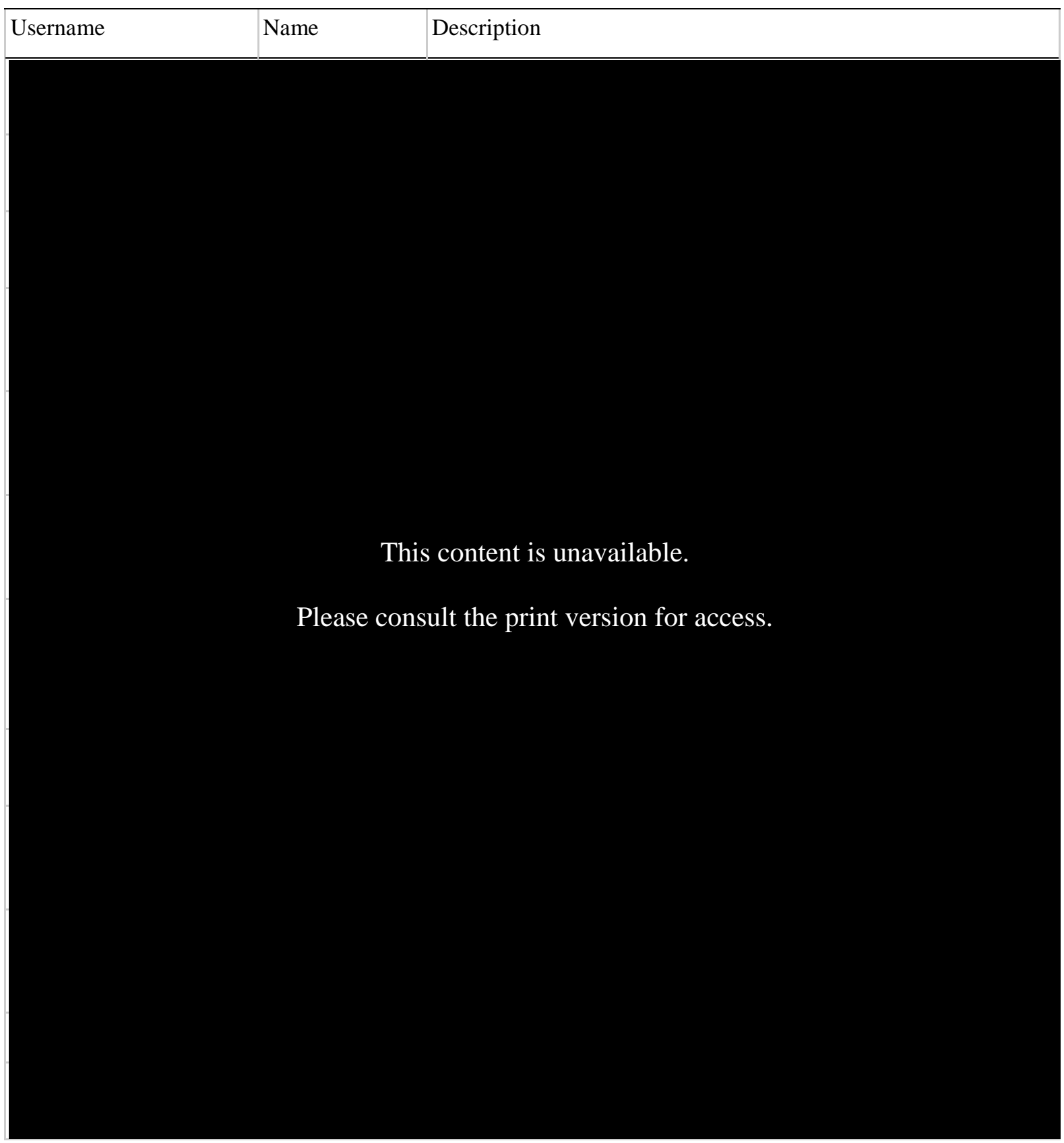




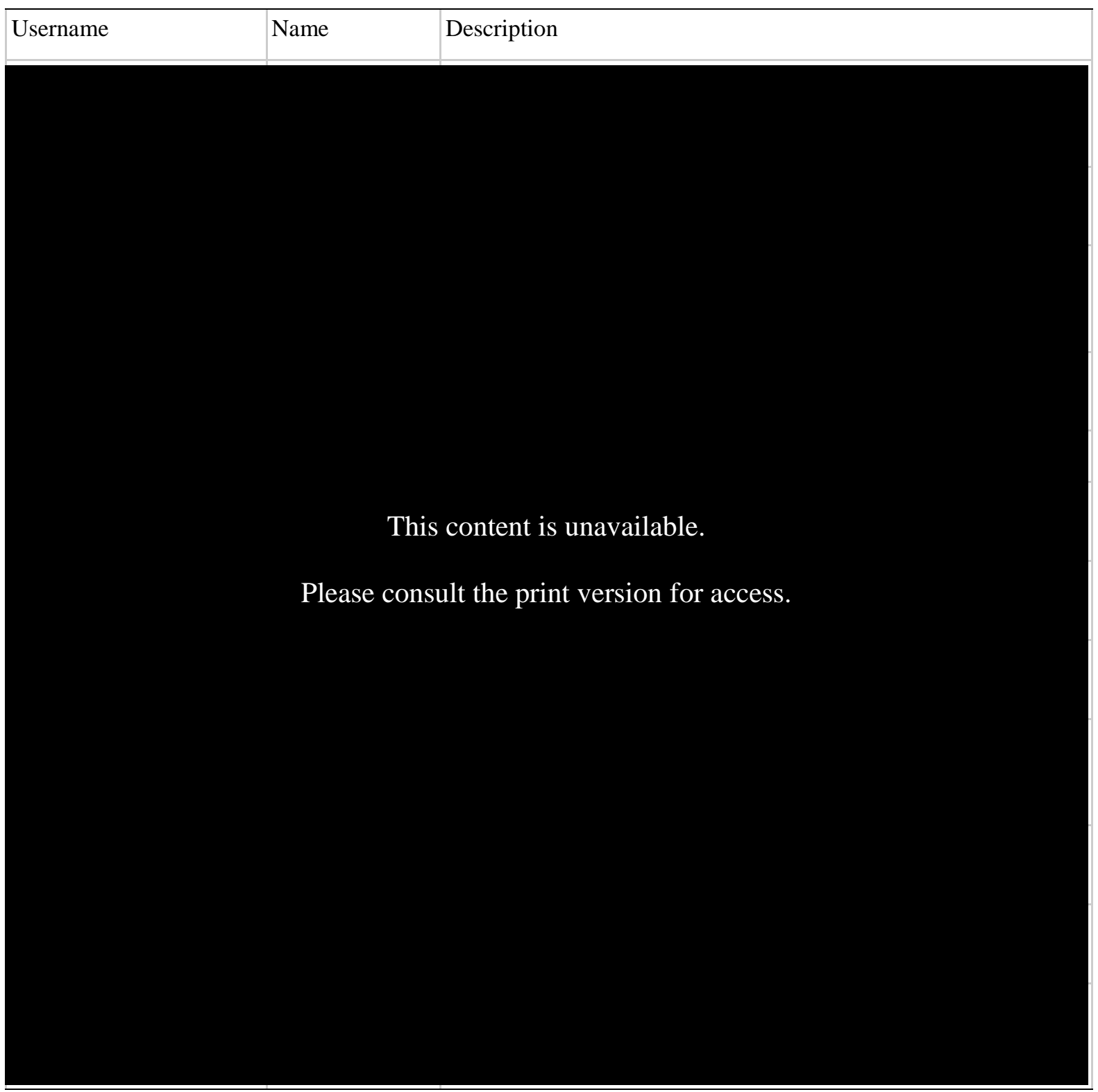

*Denotes individual or organisation listed in the demogblog.com Climate Misinformation Database. Unicode emoticons $(<\mathrm{U}+\ldots)$ not displayed in table.

Table F.2

GM sceptic accounts and descriptions

\begin{tabular}{|l|l|}
\hline Username & Name \\
\hline This content is unavailable. \\
Please consult the print version for access. \\
\\
The \\
\hline
\end{tabular}


This content is unavailable.

Please consult the print version for access. 
This content is unavailable.

Please consult the print version for access.

Unicode emoticons $(<\mathrm{U}+\ldots)$ not displayed in table.

Table F.3

Vaccine sceptic accounts and descriptions

\begin{tabular}{|l|l|l}
\hline Username & Name & Description
\end{tabular}

This content is unavailable.

Please consult the print version for access. 
This content is unavailable.

Please consult the print version for access. 
This content is unavailable.

Please consult the print version for access. 
This content is unavailable.

Please consult the print version for access.

Unicode emoticons $(<\mathrm{U}+\ldots)$ not displayed in table. 



\section{Appendix G}

\section{Study 5 full parameter estimates}

This appendix details the regression coefficients for all paths in the main model predicting rejection of science across the five issues covered in Study 5 (Table G.1) as well as estimates of all indirect effects in the model (Table G.2). Here I also report all regression coefficients for the model predicting the second order science acceptance latent variable (Table G.3). 
Table G.1

Standardised estimates of main model regression parameters (Study 5, US sample)

\begin{tabular}{|c|c|c|c|c|c|c|c|c|c|c|c|}
\hline & Religiosity & Religiosity & $\begin{array}{l}\text { Free } \\
\text { market }\end{array}$ & $\begin{array}{l}\text { Conspiracy } \\
\text { mentality }\end{array}$ & $\begin{array}{l}\text { Scientists' } \\
\text { credibility }\end{array}$ & $\begin{array}{l}\text { Science } \\
\text { literacy }\end{array}$ & $\begin{array}{l}\text { Climate } \\
\text { change }\end{array}$ & GM Food & Vaccination & Fluoridation & Evolution \\
\hline Age & $\begin{array}{l}.12 * * * \\
(.03)\end{array}$ & $\begin{array}{l}.01 \\
(.02)\end{array}$ & $\begin{array}{l}.00 \\
(.02)\end{array}$ & $\begin{array}{l}-.12^{*} \\
(.03)\end{array}$ & $\begin{array}{l}-.04 \\
(.02)\end{array}$ & $\begin{array}{l}.14^{*} \\
(.02)\end{array}$ & $\begin{array}{l}-.08^{*} \\
(.02)\end{array}$ & $\begin{array}{l}-.10^{*} \\
(.02)\end{array}$ & $\begin{array}{l}-.14 * * * \\
(.02)\end{array}$ & $\begin{array}{l}-.01 \\
(.03)\end{array}$ & $\begin{array}{l}-.01 \\
(.02)\end{array}$ \\
\hline Gender & $\begin{array}{l}-.09 * \\
(.14)\end{array}$ & $\begin{array}{l}.15^{* * * *} \\
(.1)\end{array}$ & $\begin{array}{l}.00 \\
(.08)\end{array}$ & $\begin{array}{l}-.09 * \\
(.13)\end{array}$ & $\begin{array}{l}-.01 \\
(.09)\end{array}$ & $\begin{array}{l}.19 * * * \\
(.07)\end{array}$ & $\begin{array}{l}.09 * \\
(.09)\end{array}$ & $\begin{array}{l}.08^{*} \\
(.11)\end{array}$ & $\begin{array}{l}.01 \\
(.09)\end{array}$ & $\begin{array}{l}.10^{*} \\
(.12)\end{array}$ & $\begin{array}{l}.05 \\
(.11)\end{array}$ \\
\hline RWA & $\begin{array}{l}.61 * * * \\
(.10)\end{array}$ & $\begin{array}{l}.53 * * * \\
(.07)\end{array}$ & $\begin{array}{l}.47 * * * \\
(.09)\end{array}$ & $\begin{array}{l}.24 * * * \\
(.10)\end{array}$ & $\begin{array}{l}-.54 * * * \\
(.08)\end{array}$ & $\begin{array}{l}-.41 * * * \\
(.06)\end{array}$ & $\begin{array}{l}-.03 \\
(.10)\end{array}$ & $\begin{array}{l}-.07 \\
(.11)\end{array}$ & $\begin{array}{l}.05 \\
(.10)\end{array}$ & $\begin{array}{l}.03 \\
(.13)\end{array}$ & $\begin{array}{l}-.11 \\
(.12)\end{array}$ \\
\hline SDO & $\begin{array}{l}-.18 * * * \\
(.10)\end{array}$ & $\begin{array}{l}.24 * * * \\
.06)\end{array}$ & $\begin{array}{l}.21 * * * \\
(.06)\end{array}$ & $\begin{array}{l}.09 \\
(.09)\end{array}$ & $\begin{array}{l}-.14^{*} \\
(.07)\end{array}$ & $\begin{array}{l}.03 \\
(.05)\end{array}$ & $\begin{array}{l}-.17 * \\
(.07)\end{array}$ & $\begin{array}{l}.03 \\
(.07)\end{array}$ & $\begin{array}{l}-.03 \\
(.07)\end{array}$ & $\begin{array}{l}-.01 \\
(.09)\end{array}$ & $\begin{array}{l}.11 \\
(.08)\end{array}$ \\
\hline Religiosity & & & & & & & $\begin{array}{l}.04 \\
(.04)\end{array}$ & $\begin{array}{l}-.12^{*} \\
(.04)\end{array}$ & $\begin{array}{l}.08 \\
(.03)\end{array}$ & $\begin{array}{l}.04 \\
(.04)\end{array}$ & $\begin{array}{l}-.27 * * * \\
(.05)\end{array}$ \\
\hline Conservatism & & & & & & & $\begin{array}{l}-.20 * * * \\
(.04)\end{array}$ & $\begin{array}{l}.00 \\
(.05)\end{array}$ & $\begin{array}{l}-.07 \\
(.04)\end{array}$ & $\begin{array}{l}-.04 \\
(.05)\end{array}$ & $\begin{array}{l}-.08 \\
(.06)\end{array}$ \\
\hline Free market & & & & & & & $\begin{array}{l}-.21 * * * \\
(.07)\end{array}$ & $\begin{array}{l}.02 \\
(.07)\end{array}$ & $\begin{array}{l}-.02 \\
(.06)\end{array}$ & $\begin{array}{l}-.05 \\
(.09)\end{array}$ & $\begin{array}{l}-.11 \\
(.08)\end{array}$ \\
\hline $\begin{array}{l}\text { Conspiracy } \\
\text { mentality }\end{array}$ & & & & & & & $\begin{array}{l}-.04 \\
(.04)\end{array}$ & $\begin{array}{l}-.25 * * * \\
(.04)\end{array}$ & $\begin{array}{l}-.09 * \\
(.03)\end{array}$ & $\begin{array}{l}-.26 * * * \\
(.05)\end{array}$ & $\begin{array}{l}-.02 \\
(.04)\end{array}$ \\
\hline $\begin{array}{l}\text { Scientists' } \\
\text { credibility }\end{array}$ & & & & & & & $\begin{array}{l}.32 * * * \\
(.07)\end{array}$ & $\begin{array}{l}.18^{*} \\
(.07)\end{array}$ & $\begin{array}{l}.46 * * * \\
(.06)\end{array}$ & $\begin{array}{l}.28 * * * \\
(.08)\end{array}$ & $\begin{array}{l}.29 * * * \\
(.08)\end{array}$ \\
\hline $\begin{array}{l}\text { Science } \\
\text { literacy }\end{array}$ & & & & & & & $\begin{array}{l}.01 \\
(.10)\end{array}$ & $\begin{array}{l}.25 * * * \\
(.12)\end{array}$ & $\begin{array}{l}.22 * * * \\
(.10)\end{array}$ & $\begin{array}{l}.11 \\
(.13)\end{array}$ & $\begin{array}{l}.04 \\
.13)\end{array}$ \\
\hline
\end{tabular}

Note. Standard error in parentheses.

$* p<.05, * * p<.01, * * * p<.00$ 
Table G.2

Indirect, direct and total effects in main model (Study 5, US sample)

\begin{tabular}{|c|c|c|c|c|c|}
\hline Dependent variable & Predictor & Mediator & Beta & $b$ & $95 \mathrm{CI}$ \\
\hline \multirow[t]{16}{*}{ Climate change } & RWA & Religiosity & .03 & .03 & {$[-.04, .12]$} \\
\hline & & Conservatism & -.10 & -.13 & {$[-.21,-.06]$} \\
\hline & & Free market ideology & -.10 & -.12 & {$[-.22,-.06]$} \\
\hline & & Conspiracy mentality & -.01 & -.01 & {$[-.05, .01]$} \\
\hline & & Scientists' credibility & -.17 & -.22 & {$[-.33,-. .13]$} \\
\hline & & Science literacy & -.01 & -.01 & {$[-.06, .05]$} \\
\hline & & Direct effect & -.03 & -.03 & {$[-.23, .17]$} \\
\hline & & Total effect & -.39 & -.50 & {$[-.64,-.36]$} \\
\hline & SDO & Religiosity & -.01 & -.01 & {$[-.04, .01]$} \\
\hline & & Conservatism & -.05 & -.05 & {$[-.09,-.02]$} \\
\hline & & Free market ideology & -.04 & -.05 & {$[-.10,-.02]$} \\
\hline & & Conspiracy mentality & .00 & .00 & {$[-.03, .002]$} \\
\hline & & Scientists' credibility & -.04 & -.05 & {$[-.12,-.01]$} \\
\hline & & Science literacy & .00 & .00 & {$[-.01, .02]$} \\
\hline & & Direct effect & -.17 & -.20 & {$[-.35,-.06]$} \\
\hline & & Total effect & -.32 & -.37 & {$[-.51,-.24]$} \\
\hline \multirow[t]{16}{*}{ GM Food } & RWA & Religiosity & -.07 & -.10 & {$[-.19,-.02]$} \\
\hline & & Conservatism & .00 & .00 & {$[-.07, .08]$} \\
\hline & & Free market ideology & .01 & .01 & {$[-.06, .08]$} \\
\hline & & Conspiracy mentality & -.06 & -.08 & {$[-.14,-.03]$} \\
\hline & & Scientists' credibility & -.10 & -.13 & {$[-.23,-.03]$} \\
\hline & & Science literacy & -.10 & -.14 & {$[-.23,-.07]$} \\
\hline & & Direct effect & -.07 & -.09 & {$[-.30, .12]$} \\
\hline & & Total effect & -.39 & -.52 & {$[-.67,-.36]$} \\
\hline & SDO & Religiosity & .02 & .03 & {$[.005, .07]$} \\
\hline & & Conservatism & .00 & .00 & {$[-.03, .03]$} \\
\hline & & Free market ideology & .00 & .01 & {$[-.02, .04]$} \\
\hline & & Conspiracy mentality & -.02 & -.03 & {$[-.07, .01]$} \\
\hline & & Scientists' credibility & -.02 & -.03 & {$[-.08,-.005]$} \\
\hline & & Science literacy & .01 & .01 & {$[-.03, .06]$} \\
\hline & & Direct effect & .03 & .04 & {$[-.10, .17]$} \\
\hline & & Total effect & .02 & .02 & {$[-.12, .18]$} \\
\hline \multirow[t]{4}{*}{ Vaccination } & RWA & Religiosity & .05 & .05 & {$[-.01, .12]$} \\
\hline & & Conservatism & -.04 & -.04 & {$[-.11, .03]$} \\
\hline & & Free market ideology & -.01 & -.01 & {$[-.07, .04]$} \\
\hline & & Conspiracy mentality & -.02 & -.02 & {$[-.06,-.002]$} \\
\hline
\end{tabular}




\begin{tabular}{|c|c|c|c|c|c|}
\hline & & Scientists' credibility & -.25 & -.27 & {$[-.38,-.18]$} \\
\hline & & Science literacy & -.09 & -.10 & {$[-.17,-.04]$} \\
\hline & & Direct effect & .05 & .05 & {$[-.13, .25]$} \\
\hline & & Total effect & -.31 & -.34 & {$[-.47,-.19]$} \\
\hline & SDO & Religiosity & -.01 & -.01 & {$[-.04, .001]$} \\
\hline & & Conservatism & -.02 & -.02 & {$[-.05, .01]$} \\
\hline & & Free market ideology & -.01 & -.01 & {$[-.03, .02]$} \\
\hline & & Conspiracy mentality & -.01 & -.01 & {$[-.03, .001]$} \\
\hline & & Scientists' credibility & -.06 & -.06 & {$[-.13,-.01]$} \\
\hline & & Science literacy & .01 & .01 & {$[-.02, .04]$} \\
\hline & & Direct effect & -.03 & -.03 & {$[-.17, .09]$} \\
\hline & & Total effect & -.13 & -.13 & {$[-.26,-.01]$} \\
\hline \multirow[t]{16}{*}{ Fluoridation } & RWA & Religiosity & .02 & .03 & {$[-.05, .13]$} \\
\hline & & Conservatism & -.02 & -.03 & {$[-.12, .06]$} \\
\hline & & Free market ideology & -.02 & -.03 & {$[-.13, .05]$} \\
\hline & & Conspiracy mentality & -.06 & -.09 & {$[-.16,-.04]$} \\
\hline & & Scientists' credibility & -.15 & -.22 & {$[-.34,-.11]$} \\
\hline & & Science literacy & -.04 & -.07 & {$[-.15, .001]$} \\
\hline & & Direct effect & .03 & .04 & {$[-.20, .30]$} \\
\hline & & Total effect & -.24 & -.36 & {$[-.55,-.17]$} \\
\hline & SDO & Religiosity & -.01 & -.01 & {$[-.05, .01]$} \\
\hline & & Conservatism & -.01 & -.01 & {$[-.05, .02]$} \\
\hline & & Free market ideology & -.01 & -.01 & {$[-.06, .02]$} \\
\hline & & Conspiracy mentality & -.02 & -.03 & {$[-.09, .01]$} \\
\hline & & Scientists' credibility & -.04 & -.05 & {$[-.11,-.01]$} \\
\hline & & Science literacy & .00 & .00 & {$[-.01, .04]$} \\
\hline & & Direct effect & -.01 & -.02 & {$[-.20, .16]$} \\
\hline & & Total effect & -.10 & -.13 & {$[-.32, .05]$} \\
\hline \multirow[t]{11}{*}{ Evolution } & RWA & Religiosity & -.16 & -.24 & {$[-.35,-.14]$} \\
\hline & & Conservatism & -.04 & -.06 & {$[-.15, .03]$} \\
\hline & & Free market ideology & -.05 & -.07 & {$[-.16,-.004]$} \\
\hline & & Conspiracy mentality & .00 & -.01 & {$[-.04, .02]$} \\
\hline & & Scientists' credibility & -.16 & -.23 & {$[-.35,-.12]$} \\
\hline & & Science literacy & -.02 & -.02 & {$[-.11, .05]$} \\
\hline & & Direct effect & -.11 & -.16 & {$[-.41, .09]$} \\
\hline & & Total effect & -.55 & -.79 & {$[-.97,-.62]$} \\
\hline & SDO & Religiosity & .05 & .06 & {$[.02, .13]$} \\
\hline & & Conservatism & -.02 & -.02 & {$[-.07, .01]$} \\
\hline & & Free market ideology & -.02 & -.03 & {$[-.07,-.004]$} \\
\hline
\end{tabular}




\begin{tabular}{|l|l|r|r|r|}
\hline & Conspiracy mentality & .00 & .00 & {$[-.02, .01]$} \\
\hline & Scientists' credibility & $\mathbf{- . 0 4}$ & $\mathbf{- . 0 5}$ & {$[-.12,-.01]$} \\
\hline & Science literacy & .00 & .00 & {$[-.01, .03]$} \\
\hline & Direct effect & .11 & .14 & {$[-.01, .29]$} \\
\hline & Total effect & .07 & .10 & {$[-.08, .26]$} \\
\hline
\end{tabular}

Standardized (Beta) and unstandardized $(b)$ effects shown. Bias-corrected bootstrapped $95 \%$ confidence intervals (5,000 samples) are for $b$ values. Bold values denote significant effects (confidence interval does not include zero) of predictors 
Table G.3

Standardised estimates of regression parameters in second-order science acceptance model (Study 5, US sample)

\begin{tabular}{|c|c|c|c|c|c|c|c|}
\hline & Religiosity & Conservatism & Free market & $\begin{array}{l}\text { Conspiracy } \\
\text { mentality }\end{array}$ & $\begin{array}{l}\text { Scientists' } \\
\text { credibility }\end{array}$ & Science literacy & $\begin{array}{l}\text { Science } \\
\text { acceptance }\end{array}$ \\
\hline Age & $\begin{array}{l}.12 * * * \\
(.03)\end{array}$ & $\begin{array}{l}.01 \\
(.02)\end{array}$ & $\begin{array}{l}.00 \\
(.02)\end{array}$ & $\begin{array}{l}-.12 * \\
(.03)\end{array}$ & $\begin{array}{l}-.04 \\
(.02)\end{array}$ & $\begin{array}{l}.14^{*} \\
(.02)\end{array}$ & $\begin{array}{l}-.11 * * * \\
(.01)\end{array}$ \\
\hline Gender & $\begin{array}{l}-.09 * \\
(.14)\end{array}$ & $\begin{array}{l}.15^{* * * *} \\
(.10)\end{array}$ & $\begin{array}{l}.00 \\
(.09)\end{array}$ & $\begin{array}{l}-.09 * \\
(.13)\end{array}$ & $\begin{array}{l}-.01 \\
(.09)\end{array}$ & $\begin{array}{l}.19 * * * \\
(.07)\end{array}$ & $\begin{array}{l}.09 * \\
(.06)\end{array}$ \\
\hline RWA & $\begin{array}{l}.61 * * * \\
(.10)\end{array}$ & $\begin{array}{l}.53 * * * \\
.07)\end{array}$ & $\begin{array}{l}.47 * * * \\
(.09)\end{array}$ & $\begin{array}{l}.24 * * * \\
(.10)\end{array}$ & $\begin{array}{l}-.54 * * * \\
(.08)\end{array}$ & $\begin{array}{l}-.41 * * * \\
(.05)\end{array}$ & $\begin{array}{l}-.03 \\
(.07)\end{array}$ \\
\hline SDO & $\begin{array}{l}-.18 * * * \\
(.10)\end{array}$ & $\begin{array}{l}.24 * * * \\
(.06)\end{array}$ & $\begin{array}{l}.21 * * * \\
(.06)\end{array}$ & $\begin{array}{l}.10 \\
(.08)\end{array}$ & $\begin{array}{l}-.14 * \\
(.07)\end{array}$ & $\begin{array}{l}.03 \\
(.05)\end{array}$ & $\begin{array}{l}-.03 \\
(.05)\end{array}$ \\
\hline Religiosity & & & & & & & $\begin{array}{l}-.06 \\
(.02)\end{array}$ \\
\hline Conservatism & & & & & & & $\begin{array}{l}-.11 \\
(.03)\end{array}$ \\
\hline Free market & & & & & & & $\begin{array}{l}-.10 \\
(.04)\end{array}$ \\
\hline Conspiracy mentality & & & & & & & $\begin{array}{l}-.18 * * * \\
(.03)\end{array}$ \\
\hline Scientists' credibility & & & & & & & $\begin{array}{l}.46 * * * \\
(.05)\end{array}$ \\
\hline Science literacy & & & & & & & $\begin{array}{l}.20 * * * \\
(.07)\end{array}$ \\
\hline
\end{tabular}




\section{Appendix H}

\section{Study 6 full parameter estimates}

This appendix details the regression coefficients for all paths in the main model predicting rejection of science across the five issues covered in Study 6 (Table H.1) as well as estimates of all indirect effects in the model (Table H.2). Here I also report all regression coefficients for the model predicting the second order science acceptance latent variable (Table H.3). 
Table H.1

Standardised estimates of main model regression parameters (Study 6, NZ sample)

\begin{tabular}{|c|c|c|c|c|c|c|c|c|c|c|c|}
\hline & Religiosity & Conservatism & $\begin{array}{l}\text { Free } \\
\text { market }\end{array}$ & $\begin{array}{l}\text { Conspiracy } \\
\text { mentality }\end{array}$ & $\begin{array}{l}\text { Scientists' } \\
\text { credibility }\end{array}$ & $\begin{array}{l}\text { Science } \\
\text { literacy }\end{array}$ & $\begin{array}{l}\text { Climate } \\
\text { change }\end{array}$ & $\begin{array}{l}\text { GM } \\
\text { Food }\end{array}$ & Vaccination & Fluoridation & Evolution \\
\hline Age & $\begin{array}{l}.07 * * * \\
(.01)\end{array}$ & $\begin{array}{l}.04 * * * \\
(.00)\end{array}$ & $\begin{array}{l}-.09 * * * \\
(.00)\end{array}$ & $\begin{array}{l}-.04 * * * \\
(.01)\end{array}$ & $\begin{array}{l}-.04 * * * \\
(.00)\end{array}$ & $\begin{array}{l}.06 * * * \\
.00)\end{array}$ & $\begin{array}{l}-.08 * * * \\
(.00)\end{array}$ & $\begin{array}{l}-.03 * \\
(.00)\end{array}$ & $\begin{array}{l}.00 \\
.00)\end{array}$ & $\begin{array}{l}.06 * * * \\
(.00)\end{array}$ & $\begin{array}{l}.05 * * * \\
(.00)\end{array}$ \\
\hline Gender & $\begin{array}{l}-.07 * * * \\
(.03)\end{array}$ & $\begin{array}{l}.03 * * * \\
(.02)\end{array}$ & $\begin{array}{l}-.07 * * * \\
(.02)\end{array}$ & $\begin{array}{l}-.14 * * * \\
(.03)\end{array}$ & $\begin{array}{l}.1 * * * \\
(.02)\end{array}$ & $\begin{array}{l}.29 * * * \\
(.02)\end{array}$ & $\begin{array}{l}.02 \\
(.03)\end{array}$ & $\begin{array}{l}.07 * * * \\
(.03)\end{array}$ & $\begin{array}{l}-.01 \\
(.02)\end{array}$ & $\begin{array}{l}.04 * * * \\
(.03)\end{array}$ & $\begin{array}{l}.02 * \\
(.03)\end{array}$ \\
\hline RWA & $\begin{array}{l}.36 * * * \\
(.04)\end{array}$ & $\begin{array}{l}.59 * * * \\
(.03)\end{array}$ & $\begin{array}{l}.36 * * * \\
(.04)\end{array}$ & $\begin{array}{l}.36 * * * \\
(.04)\end{array}$ & $\begin{array}{l}-.34 * * * \\
(.03)\end{array}$ & $\begin{array}{l}-.34 * * * * \\
(.02)\end{array}$ & $\begin{array}{l}-.06^{* * * *} \\
(.05)\end{array}$ & $\begin{array}{l}-.03 \\
(.05)\end{array}$ & $\begin{array}{l}.13^{* * * *} \\
(.03)\end{array}$ & $\begin{array}{l}.03 \\
(.05)\end{array}$ & $\begin{array}{l}-.08 * * * \\
(.04)\end{array}$ \\
\hline SDO & $\begin{array}{l}-.16^{* * * *} \\
(.03)\end{array}$ & $\begin{array}{l}.22 * * * \\
(.02)\end{array}$ & $\begin{array}{l}.30 * * * \\
(.02)\end{array}$ & $\begin{array}{l}.08 * * * \\
(.03)\end{array}$ & $\begin{array}{l}-.15^{* * * *} \\
(.02)\end{array}$ & $\begin{array}{l}.02 \\
(.01)\end{array}$ & $\begin{array}{l}-.16^{* * * *} \\
(.02)\end{array}$ & $\begin{array}{l}.03 \\
(.02)\end{array}$ & $\begin{array}{l}-.09^{* * * *} \\
(.01)\end{array}$ & $\begin{array}{l}-.02 \\
(.02)\end{array}$ & $\begin{array}{l}.02 \\
(.02)\end{array}$ \\
\hline Religiosity & & & & & & & $\begin{array}{l}-.02 \\
(.01)\end{array}$ & $\begin{array}{l}-.06 * * * \\
(.01)\end{array}$ & $\begin{array}{l}-.05^{* * *} \\
(.01)\end{array}$ & $\begin{array}{l}.00 \\
(.01)\end{array}$ & $\begin{array}{l}-.50 * * * \\
(.01)\end{array}$ \\
\hline Conservatism & & & & & & & $\begin{array}{l}-.11^{* * * *} \\
(.02)\end{array}$ & $\begin{array}{l}-.01 \\
(.02)\end{array}$ & $\begin{array}{l}.01 \\
(.01)\end{array}$ & $\begin{array}{l}.05^{*} \\
(.02)\end{array}$ & $\begin{array}{l}-.09 * * * \\
(.02)\end{array}$ \\
\hline Free market & & & & & & & $\begin{array}{l}-.28 * * * \\
(.02)\end{array}$ & $\begin{array}{l}.12 * * * \\
(.02)\end{array}$ & $\begin{array}{l}.01 \\
(.01)\end{array}$ & $\begin{array}{l}-.03 \\
(.02)\end{array}$ & $\begin{array}{l}.02 \\
(.01)\end{array}$ \\
\hline $\begin{array}{l}\text { Conspiracy } \\
\text { mentality }\end{array}$ & & & & & & & $\begin{array}{l}-.05^{* * * *} \\
(.01)\end{array}$ & $\begin{array}{l}-.29 * * * \\
(.01)\end{array}$ & $\begin{array}{l}-.18^{* * * *} \\
(.01)\end{array}$ & $\begin{array}{l}-.29 * * * \\
(.02)\end{array}$ & $\begin{array}{l}.00 \\
(.01)\end{array}$ \\
\hline $\begin{array}{l}\text { Scientists' } \\
\text { credibility }\end{array}$ & & & & & & & $\begin{array}{l}.28 * * * \\
(.02)\end{array}$ & $\begin{array}{l}.24 * * * \\
(.02)\end{array}$ & $\begin{array}{l}.39 * * * \\
(.02)\end{array}$ & $\begin{array}{l}.32 * * * \\
(.02)\end{array}$ & $\begin{array}{l}.23 * * * \\
(.02)\end{array}$ \\
\hline $\begin{array}{l}\text { Science } \\
\text { literacy }\end{array}$ & & & & & & & $\begin{array}{l}.00 \\
(.03)\end{array}$ & $\begin{array}{l}.18 * * * \\
(.03)\end{array}$ & $\begin{array}{l}.10 * * * \\
(.02)\end{array}$ & $\begin{array}{l}.10 * * * \\
(.03)\end{array}$ & $\begin{array}{l}.10 * * * \\
(.03)\end{array}$ \\
\hline
\end{tabular}


Table H.2

Indirect, direct and total effects in main model (Study 6, NZ sample)

\begin{tabular}{|c|c|c|c|c|c|}
\hline Dependent variable & Predictor & Mediator & $\beta$ & $b$ & $5 \mathrm{CI}$ \\
\hline \multirow[t]{16}{*}{ Climate change } & RWA & Religiosity & -.01 & -.02 & {$[-.04, .004]$} \\
\hline & & Conservatism & -.07 & -.16 & {$[-.21,-.11]$} \\
\hline & & Free market ideology & -.10 & -.24 & {$[-.28,-.21]$} \\
\hline & & Conspiracy mentality & -.02 & -.04 & {$[-.07,-.02]$} \\
\hline & & Scientists' credibility & -.10 & -.23 & {$[-.26,-.20]$} \\
\hline & & Science literacy & .00 & .00 & {$[-.02, .03]$} \\
\hline & & Direct effect & -.06 & -.14 & {$[-.24,-.05]$} \\
\hline & & Total effect & -.35 & -.83 & {$[-.91,-.75]$} \\
\hline & SDO & Religiosity & .00 & .00 & {$[-.001, .01]$} \\
\hline & & Conservatism & -.03 & -.04 & {$[-.05,-.02]$} \\
\hline & & Free market ideology & -.08 & -.12 & {$[-.14,-.10]$} \\
\hline & & Conspiracy mentality & .00 & -.01 & {$[-.01, .00]$} \\
\hline & & Scientists' credibility & -.04 & -.06 & {$[-.08,-.05]$} \\
\hline & & Science literacy & .00 & .00 & {$[-.002, .001]$} \\
\hline & & Direct effect & -.16 & -.23 & {$[-.28,-.18]$} \\
\hline & & Total effect & -.31 & -.45 & {$[-.49,-.40]$} \\
\hline \multirow[t]{16}{*}{ GM Food } & RWA & Religiosity & -.02 & -.04 & {$[-.06,-.02]$} \\
\hline & & Conservatism & .00 & -.01 & {$[-.06, .04]$} \\
\hline & & Free market ideology & .04 & .09 & {$[.07, .12]$} \\
\hline & & Conspiracy mentality & -.10 & -.22 & {$[-.26,-.19]$} \\
\hline & & Scientists' credibility & -.08 & -.18 & {$[-.21,-.15]$} \\
\hline & & Science literacy & -.06 & -.13 & {$[-.16,-.11]$} \\
\hline & & Direct effect & -.03 & -.07 & {$[-.17, .02]$} \\
\hline & & Total effect & -.26 & -.56 & {$[-.63,-.49]$} \\
\hline & SDO & Religiosity & .01 & .01 & {$[.01, .02]$} \\
\hline & & Conservatism & .00 & .00 & {$[-.01, .01]$} \\
\hline & & Free market ideology & .04 & .05 & {$[.03, .06]$} \\
\hline & & Conspiracy mentality & -.02 & -.03 & {$[-.04,-.02]$} \\
\hline & & Scientists' credibility & -.04 & -.05 & {$[-.06,-.04]$} \\
\hline & & Science literacy & .00 & .01 & {$[-.003, .01]$} \\
\hline & & Direct effect & .03 & .04 & {$[-.01, .08]$} \\
\hline & & Total effect & .02 & .02 & {$[-.02, .07]$} \\
\hline \multirow[t]{4}{*}{ Vaccination } & RWA & Religiosity & -.02 & -.02 & {$[-.04,-.01]$} \\
\hline & & Conservatism & .01 & .01 & {$[-.03, .04]$} \\
\hline & & Free market ideology & .00 & .00 & {$[-.01, .02]$} \\
\hline & & Conspiracy mentality & -.07 & -.08 & {$[-.10,-.07]$} \\
\hline
\end{tabular}




\begin{tabular}{|c|c|c|c|c|c|}
\hline & & Scientists' credibility & -.13 & -.17 & {$[-.19,-. .14]$} \\
\hline & & Science literacy & -.03 & -.04 & {$[-.06,-.03]$} \\
\hline & & Direct effect & .13 & .17 & {$[.11, .24]$} \\
\hline & & Total effect & -.10 & -.13 & {$[-.17,-.09]$} \\
\hline & SDO & Religiosity & .01 & .01 & {$[.002, .01]$} \\
\hline & & Conservatism & .00 & .00 & {$[-.01, .01]$} \\
\hline & & Free market ideology & .00 & .00 & {$[-.01, .01]$} \\
\hline & & Conspiracy mentality & -.01 & -.01 & {$[-.02,-.01]$} \\
\hline & & Scientists' credibility & -.06 & -.04 & {$[-.06,-.03]$} \\
\hline & & Science literacy & .00 & .00 & {$[-.001, .005]$} \\
\hline & & Direct effect & -.09 & -.07 & {$[-.10,-.04]$} \\
\hline & & Total effect & -.15 & -.11 & {$[-.14,-.09]$} \\
\hline \multirow[t]{16}{*}{ Fluoridation } & RWA & Religiosity & .00 & .00 & {$[-.02, .02]$} \\
\hline & & Conservatism & .03 & .06 & {$[.02, .11]$} \\
\hline & & Free market ideology & -.01 & -.02 & {$[-.04, .002]$} \\
\hline & & Conspiracy mentality & -.10 & -.22 & {$[-.25,-.19]$} \\
\hline & & Scientists' credibility & -.11 & -.22 & {$[-.26,-.19]$} \\
\hline & & Science literacy & -.03 & -.07 & {$[-.10,-.05]$} \\
\hline & & Direct effect & .03 & .07 & {$[-.02, .17]$} \\
\hline & & Total effect & -.19 & -.40 & {$[-.47,-.33]$} \\
\hline & SDO & Religiosity & .00 & .00 & {$[-.004, .01]$} \\
\hline & & Conservatism & .01 & .01 & {$[.004, .03]$} \\
\hline & & Free market ideology & -.01 & -.01 & {$[-.02, .00]$} \\
\hline & & Conspiracy mentality & -.02 & -.03 & {$[-.04,-.02]$} \\
\hline & & Scientists' credibility & -.05 & -.06 & {$[-.08,-.04]$} \\
\hline & & Science literacy & .00 & .00 & {$[-.004, .01]$} \\
\hline & & Direct effect & -.02 & -.03 & {$[-.07, .01]$} \\
\hline & & Total effect & -.09 & -.11 & {$[-.15,-.06]$} \\
\hline \multirow[t]{11}{*}{ Evolution } & RWA & Religiosity & -.18 & -.42 & {$[-.47,-.37]$} \\
\hline & & Conservatism & -.05 & -.12 & {$[-.16,-.07]$} \\
\hline & & Free market ideology & .01 & .02 & {$[-.004, .04]$} \\
\hline & & Conspiracy mentality & .00 & .00 & {$[-.02, .02]$} \\
\hline & & Scientists' credibility & -.08 & -.18 & {$[-.21,-.15]$} \\
\hline & & Science literacy & -.04 & -.08 & {$[-.11,-.06]$} \\
\hline & & Direct effect & -.08 & -.19 & {$[-.27,-. .10]$} \\
\hline & & Total effect & -.42 & -.97 & {$[-1.05,-.90]$} \\
\hline & SDO & Religiosity & .08 & .11 & {$[.09, .14]$} \\
\hline & & Conservatism & -.02 & -.03 & {$[-.04,-.02]$} \\
\hline & & Free market ideology & .01 & .01 & {$[-.002, .02]$} \\
\hline
\end{tabular}




\begin{tabular}{|l|l|r|r|r|}
\hline & Conspiracy mentality & .00 & .00 & {$[-.004, .006]$} \\
\hline & Scientists' credibility & $\mathbf{- . 0 3}$ & $\mathbf{- . 0 5}$ & {$[-.06,-.04]$} \\
\hline & Science literacy & .00 & .00 & {$[-.002, .01]$} \\
\hline & Direct effect & .02 & .02 & {$[-.02, .06]$} \\
\hline & Total effect & $\mathbf{. 0 5}$ & $\mathbf{. 0 7}$ & {$[.03, .12$} \\
\hline
\end{tabular}

Standardized (Beta) and unstandardized $(b)$ effects shown. Bias-corrected bootstrapped $95 \%$ confidence intervals (5,000 samples) are for $b$ values. Bold values denote significant effects (confidence interval does not include zero). 
Table H.3

Standardised estimates of regression parameters in second-order science acceptance model (Study 6, NZ sample)

\begin{tabular}{|c|c|c|c|c|c|c|c|}
\hline & Religiosity & Conservatism & Free market & $\begin{array}{l}\text { Conspiracy } \\
\text { mentality }\end{array}$ & $\begin{array}{l}\text { Scientists' } \\
\text { credibility }\end{array}$ & Science literacy & $\begin{array}{l}\text { Science } \\
\text { acceptance }\end{array}$ \\
\hline Age & $\begin{array}{l}.07 * * * \\
(.01)\end{array}$ & $\begin{array}{l}.04 * * * \\
(.00)\end{array}$ & $\begin{array}{l}-.09 * * * \\
(.00)\end{array}$ & $\begin{array}{l}-.04 * * * \\
(.01)\end{array}$ & $\begin{array}{l}-.04 * * * \\
(.00)\end{array}$ & $\begin{array}{l}.07 * * * \\
(.00)\end{array}$ & $\begin{array}{l}.01 \\
(.00)\end{array}$ \\
\hline Gender & $\begin{array}{l}-.07 * * * * \\
(.03)\end{array}$ & $\begin{array}{l}.03 * * * \\
(.02)\end{array}$ & $\begin{array}{l}-.07 * * * \\
(.02)\end{array}$ & $\begin{array}{l}-.14 * * * \\
(.03)\end{array}$ & $\begin{array}{l}.10 * * * \\
(.02)\end{array}$ & $\begin{array}{l}.29 * * * \\
(.02)\end{array}$ & $\begin{array}{l}.04 * * * \\
(.02)\end{array}$ \\
\hline RWA & $\begin{array}{l}.36 * * * \\
(.04)\end{array}$ & $\begin{array}{l}.59 * * * \\
(.03)\end{array}$ & $\begin{array}{l}.36 * * * \\
(.04)\end{array}$ & $\begin{array}{l}.36 * * * \\
(.04)\end{array}$ & $\begin{array}{l}-.34 * * * \\
(.03)\end{array}$ & $\begin{array}{l}-.34 * * * \\
(.02)\end{array}$ & $\begin{array}{l}.01 \\
(.03)\end{array}$ \\
\hline SDO & $\begin{array}{l}-.16^{* * * *} \\
(.03)\end{array}$ & $\begin{array}{l}.22 * * * \\
(.02)\end{array}$ & $\begin{array}{l}.30 * * * \\
(.02)\end{array}$ & $\begin{array}{l}.08 * * * \\
(.03)\end{array}$ & $\begin{array}{l}-.15^{* * * *} \\
(.02)\end{array}$ & $\begin{array}{l}.02 \\
(.01)\end{array}$ & $\begin{array}{l}-.06^{* * * *} \\
(.01)\end{array}$ \\
\hline Religiosity & & & & & & & $\begin{array}{l}-.18^{* * *} \\
(.01)\end{array}$ \\
\hline Conservatism & & & & & & & $\begin{array}{l}-.03 \\
(.01)\end{array}$ \\
\hline Free market & & & & & & & $\begin{array}{l}-.03 \\
(.01)\end{array}$ \\
\hline Conspiracy mentality & & & & & & & $\begin{array}{l}-.28 * * * \\
(.01)\end{array}$ \\
\hline Scientists' credibility & & & & & & & $\begin{array}{l}.46^{* * * *} \\
(.02)\end{array}$ \\
\hline Science literacy & & & & & & & $\begin{array}{l}.16 * * * \\
(.02)\end{array}$ \\
\hline
\end{tabular}

$* p<.05, * * p<.01, * * * p<.001$ 
